\title{
Experimental and Numerical Studies of Heat and Moisture Transfer in Soils at Various Conditions
}

\author{
by \\ Lam Dang \\ Bachelor of Engineering in Mechanical Engineering \\ Ryerson University, 2011 \\ Master of Applied Science in Mechanical Engineering \\ Ryerson University, 2013
}

A dissertation

presented to Ryerson University

in partial fulfillment of the

requirements for the degree of

Doctor of Philosophy

in the Program of

Mechanical Engineering

Toronto, Ontario, Canada, 2017

(C) Lam Dang, 2017 


\section{AUTHOR'S DECLARATION}

I hereby declare that I am the sole author of this dissertation. This is a true copy of the dissertation, including any required final revisions, as accepted by my examiners.

I authorize Ryerson University to lend this dissertation to other institutions or individuals for the purpose of scholarly research.

I further authorize Ryerson University to reproduce this dissertation by photocopying or by other means, in total or in part, at the request of other institutions or individuals for the purpose of scholarly research.

I understand that my dissertation may be made electronically available to the public. 


\title{
Experimental and Numerical Studies of Heat and Moisture Transfer in Soils at Various Conditions
}

\author{
Doctor of Philosophy, 2017, Lam Dang \\ Department of Mechanical and Industrial Engineering \\ Ryerson University, Toronto, Ontario, Canada, 2017
}

\begin{abstract}
The main purpose of this study is to provide a better understanding of heat and moisture transfer in soils under high-temperature $\left(>40^{\circ} \mathrm{C}\right)$ conditions. Through a numerical analysis of the experimental apparatus using COMSOL, it was found that one-dimensional formulation based on the finite volume method was sufficient to numerically study the governing partial differential equations of coupled heat and moisture transfer in soils. An existing experimental apparatus and some of its experimental procedures were improved in order to obtain more accurate test results. Based on a conservative uncertainty analysis, the maximum overall uncertainties at $95 \%$ confidence level were $15.5 \%$ for thermal conductivity and $9.20 \%$ for soil volumetric heat capacity. The maximum overall uncertainty for moisture content was estimated to be $48.6 \%$ at saturation ratio (SR) of 0.25 and reduced to $29.9 \%$ at SR of 0.5 .

The heat and moisture transfer in the soil column based on the coupled governing equations were numerically simulated to compare with the experiments done on three soil types (fine soil BC1, medium soil NB2, and coarse soil QC2) with different saturation ratios (from 0.00 to 0.70 ) under different heating conditions (mostly from $42^{\circ} \mathrm{C}$ and up). It was found that the simulations for coarser soils were less accurate to predict the moisture movements and temperature responses because the moisture could flow faster in coarser soils. The pure heat conduction model was also compared with the experiments and showed higher errors in the temperature responses $(\sim 2 \%$ minimum and $\sim 5 \%$ maximum errors) than the equations of coupled heat and moisture transfer do.
\end{abstract}


Coarser soils, because of their higher sand contents, transferred more heat during transient time when the entire soil column was still quite wet, but less heat transferred during steady-state time when a part of the soil column became dry. In conclusion, the worst percentage differences between predicted and measured temperatures range from $0.89 \%$ to $3.52 \%$, while the worst percentage differences between predicted and measured moisture contents range from $4.67 \%$ to $7.53 \%$, using the one-dimensional formulations of the theoretical model of coupled heat and moisture transfer in soils. 


\section{ACKNOWLEDGEMENTS}

I would like to express my greatest appreciation to my family and my supervisors, Dr. Wey Leong and Dr. Alan Fung, for the completion of this dissertation. My family has been by my side during my worst moments to cheer me up. Also, Drs. Leong and Fung give me excellent technical guidance, insights, and instructions to make this dissertation possible.

Furthermore, I would like to thank Drs. David Naylor and Ziad Saghir (Department of Mechanical and Industrial Engineering, Ryerson University), Dr. Huu Doan (Department of Chemical Engineering, Ryerson University), and Dr. Ibrahim Dincer (Department of Automotive, Mechanical and Manufacturing Engineering, UOIT) for serving in my dissertation examination committee.

In addition, I would like to thank Dr. Mohsen Hedayati-Dezfooli, Mr. Joseph Amankrah, and Mr. Devin Ostrom from the Department of Mechanical and Industrial Engineering at Ryerson University for creating components and tools for my experimental apparatus. 


\section{TABLE OF CONTENTS}

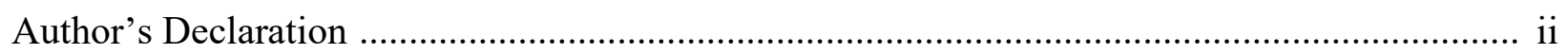

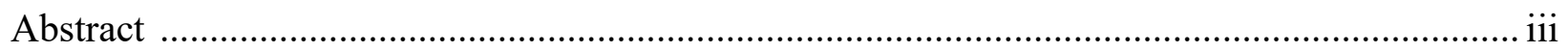

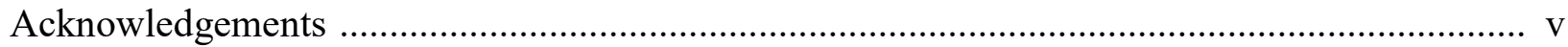

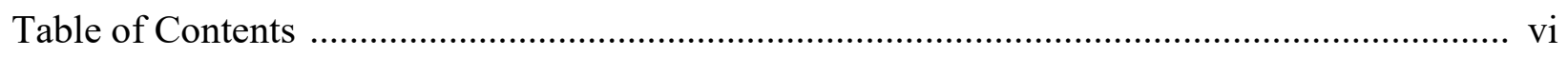

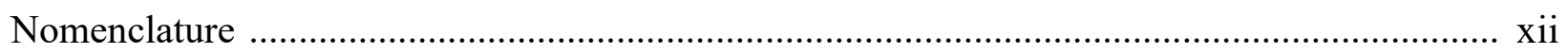

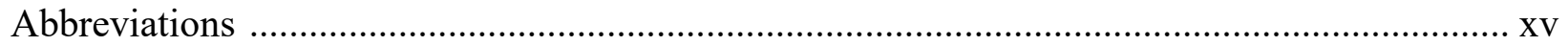

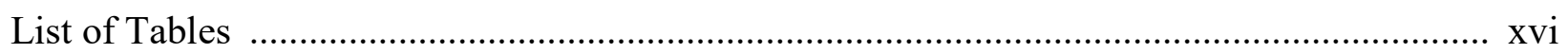

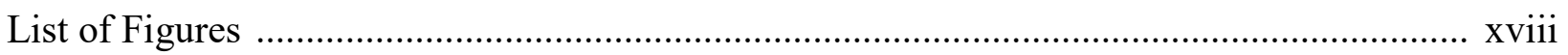

\section{CHAPTER 1 - INTRODUCTION}

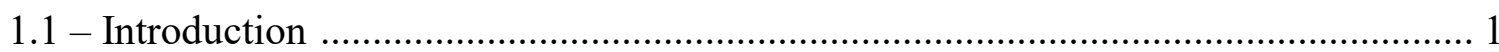

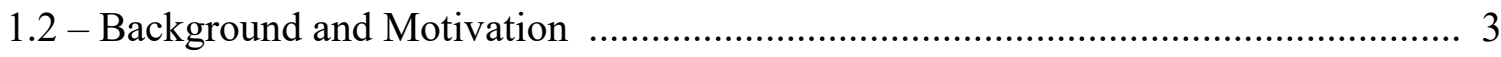

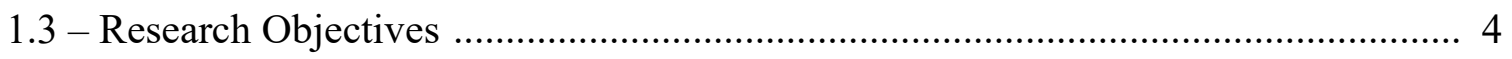

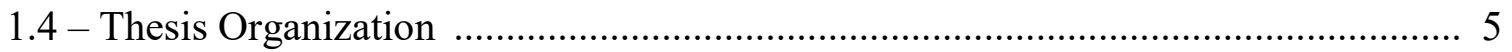

\section{CHAPTER 2 - BACKGROUND AND LITERATURE REVIEW}

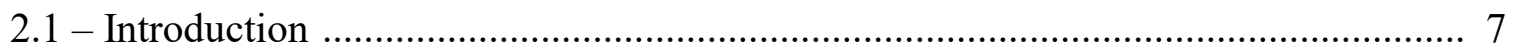

2.2 - Literature Review on the Theory of Heat and Mass Transfer .............................. 7

2.3 - Governing Equations for Coupled Heat and Moisture Transfer in Soils .............. 11

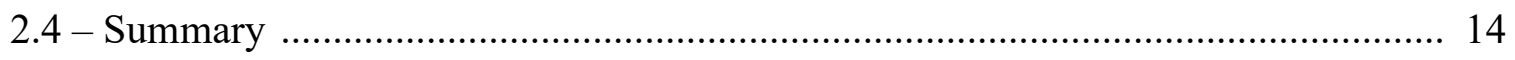




\section{CHAPTER 3 - EXPERIMENTAL APPARATUS AND METHODOLOGY}

3.1 - Experimental Apparatus .............................................................................. 16

3.2 - Improvements to the Experimental Apparatus …………................................... 17

3.3 - Experimental Techniques.......................................................................... 19

3.3.1 High Thermal Gradient Testing ……………...................................... 19

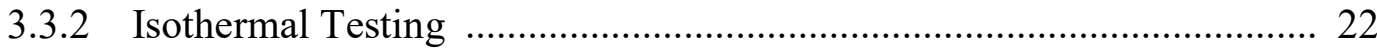

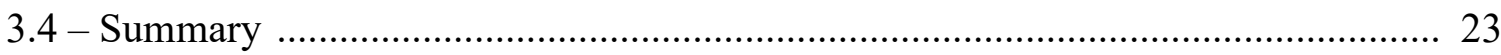

\section{CHAPTER 4 - EXPERIMENTAL RESULTS AND DISCUSSIONS}

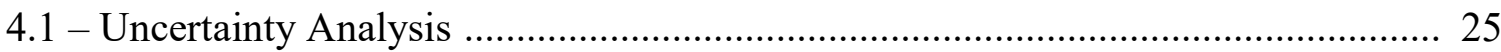

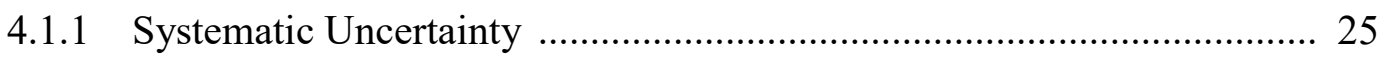

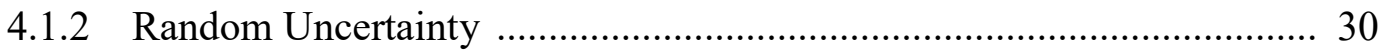

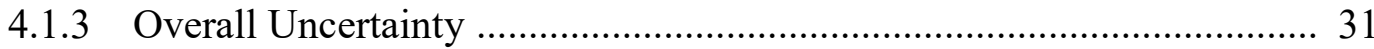

4.2 - Measured Thermal Properties of Dry QC2, NB2, and BC1 Soils ........................ 32

4.3 - Measured Thermal Properties of Wet Soils ........................................................ 35

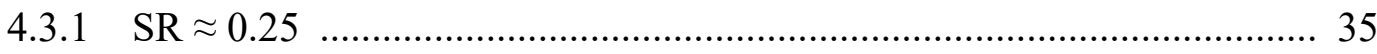

4.3.2 $\mathrm{SR} \approx 0.50$ (Field Capacity) ………………................................... 36

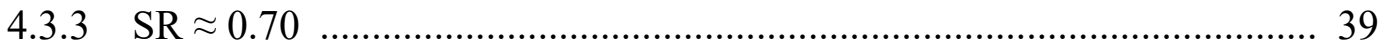

4.4 - More about Measured Thermal Properties of Soils ............................................. 40

4.5 - Thermal Storage Behaviors of Tested Soils ....................................................... 41 


\section{CHAPTER 5 - NUMERICAL FORMULATIONS USING FINITE VOLUME METHOD}

5.1 - Introduction 43

5.2 - Numerical Solution 43

5.2.1 Finite Volume Method - One-Dimensional Formulation 43

5.2.2 Finite Volume Method - Axisymmetric Formulation 50

5.3 - Development of Computer Codes 54

5.3.1 Solution Method for One-Dimensional Heat and Mass Transfer 54

5.3.2 Solution Method for Axisymmetric Two-Dimensional Heat and Mass Transfer

\section{CHAPTER 6 - NUMERICAL STUDY OF HEAT AND MOISTURE TRANSFER IN SOILS}

6.1 - Introduction 59

6.2 - Finite Element Simulations using COMSOL 59

6.3 - Numerical Simulations 68

6.4 - Grid Sensitivity Study and Verifications of the Matlab Codes 69

6.4.1 Grid Sensitivity Study 69

6.4.2 Verifications of the Matlab Codes 70 
6.5 - Sample Numerical Solutions

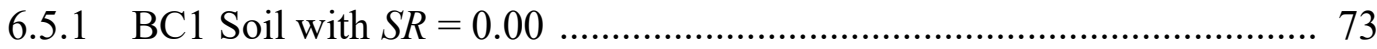

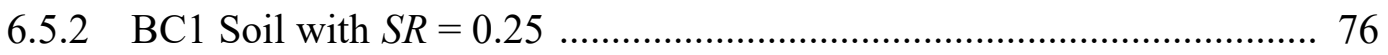

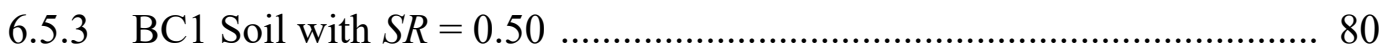

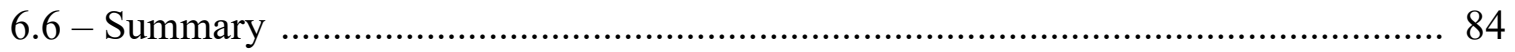

\section{CHAPTER 7 - VERIFICATION OF THE THEORETICAL MODEL OF HEAT AND MOISTURE TRANSFER IN SOILS}

7.1 - Pure Conduction Heat Transfer ....................................................................... 87

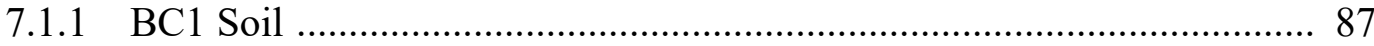

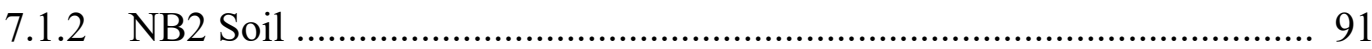

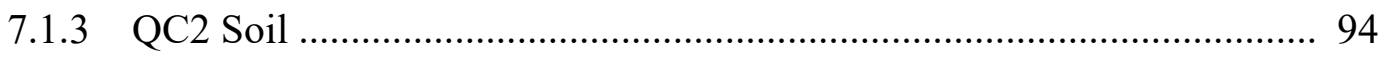

7.2 - Moisture Transfer due to Gravity …………………......................................... 96

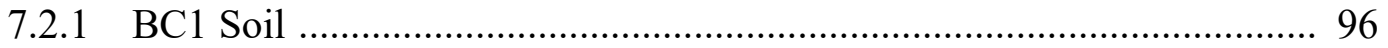

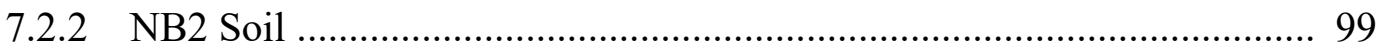

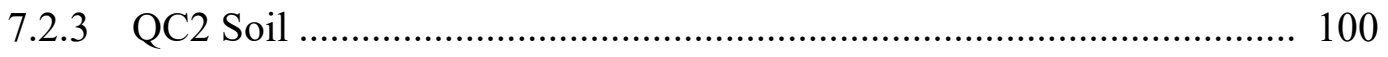

7.3 - Moisture Transfer due to Moisture Gradient …………….............................. 102

7.3.1 Horizontal Position under Room Temperature ………………........... 102

7.3.2 Vertical Position under Room Temperature …………………………... 105

7.4 - Moisture and Thermal Transfers due to Combined Moisture and Thermal Gradients 107 


\section{CHAPTER 8 - CONCLUSIONS AND RECOMMENDATIONS FOR FUTURE WORK}

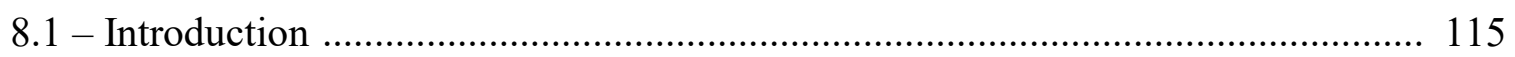

8.2 - Research Contributions.............................................................................. 116

8.3 - Concluding Remarks ......................................................................... 116

8.4 - Recommendations for Future Work ................................................................ 118

\section{APPENDICES}

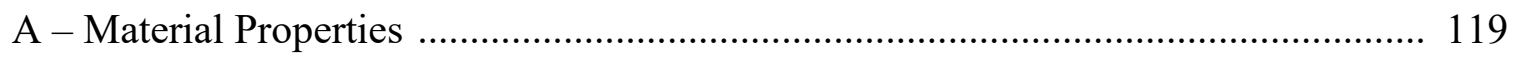

A.1 - Properties of Air and Water ...................................................................... 119

A.2 - Soil Moisture Retention ....................................................................... 120

A.3 - Hydraulic Conductivity ....................................................................... 121

A.4 - Temperature Dependency ..................................................................... 121

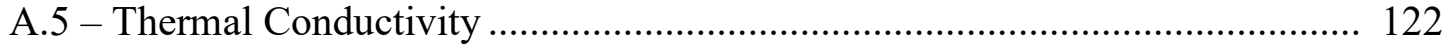

A.6 - Other Properties and Parameter Definitions ............................................... 123

A. 7 - More about COMSOL Simulations in Chapter 6 ....................................... 123

A.8 - More Thermal Properties for Section 7.1 ................................................... 125

B - More about Matlab Programming ………………............................................. 127 
C - Experimental Apparatus and Methodology by Hedayati-Dezfooli

C.1 - Experimental Apparatus ...................................................................... 130

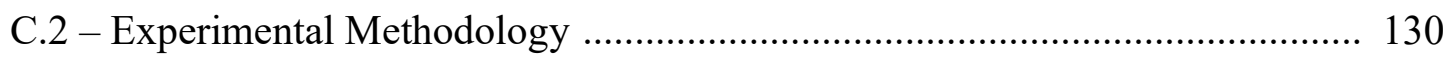

D - Derivations of some Soil Parameters ......................................................... 137

E - More Detailed Graphs ..................................................................... 141

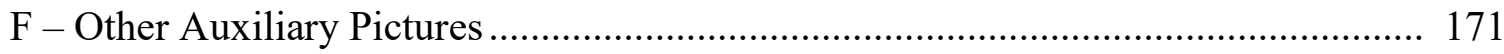

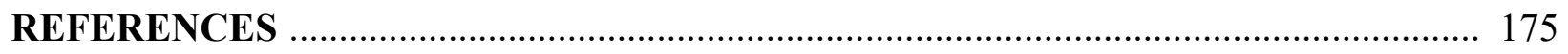




\begin{tabular}{|c|c|}
\hline C & Volumetric heat capacity $\left(\mathrm{J} / \mathrm{m}^{3} \cdot \mathrm{K}\right)$ \\
\hline$C_{p}$ & Specific heat capacity $(J / k g \cdot K)$ \\
\hline$C_{T v}, C_{T m}$ & Thermal vapor and total moisture capacitances $\left(K^{-1}\right)$ \\
\hline$C_{\psi T}, C_{T T}$ & Matric $\left(\mathrm{J} / \mathrm{m}^{4}\right)$ and thermal $\left(\mathrm{J} / \mathrm{m}^{3} \cdot \mathrm{K}\right)$ volumetric heat capacitances \\
\hline$C_{\psi r}, C_{\psi m}$ & Matric vapor and total moisture capacitance terms $\left(\mathrm{m}^{-1}\right)$ \\
\hline$D_{v a}$ & Molecular diffusivity of water vapor in air $\left(\mathrm{m}^{2} / \mathrm{s}\right)$ \\
\hline$D_{T}$ & Thermal diffusivity $\left(\mathrm{m}^{2} / \mathrm{s}\right)$ \\
\hline$D_{\theta}$ & Isothermal moisture diffusivity $\left(\mathrm{m}^{2} / \mathrm{s}\right)$ \\
\hline$D_{T v}, D_{T m}$ & Thermal vapor and total moisture diffusivities $\left(\mathrm{m}^{2} / \mathrm{s} \cdot \mathrm{K}\right)$ \\
\hline$D_{\psi \nu}, D_{\psi / m}$ & Matric vapor and total moisture diffusivities $\left(\mathrm{m}^{2} / \mathrm{s}\right)$ \\
\hline$D_{\psi T}$ & Matric potential heat diffusivity $\left(\mathrm{W} / \mathrm{m}^{2}\right)$ \\
\hline$f(), f$ & Correction and interpolation factors \\
\hline$g$ & Gravitational acceleration $\left(=9.804 \mathrm{~m} / \mathrm{s}^{2}\right)$ \\
\hline$g_{i}$ & Shape factor used in estimating soil's thermal conductivity \\
\hline$h_{f g}$ & Latent heat of vaporization $(\mathrm{J} / \mathrm{kg})$ \\
\hline K & Hydraulic conductivity of soil $(\mathrm{m} / \mathrm{s})$ \\
\hline$k$ & Thermal conductivity $(\mathrm{W} / \mathrm{m} \cdot \mathrm{K})$ \\
\hline$\dot{m}$ & Mass flux $\left(\mathrm{kg} / \mathrm{m}^{2} \cdot \mathrm{s}\right)$ \\
\hline$P$ & Total pressure in pore spaces $(\mathrm{Pa})$ \\
\hline$P_{v}$ & Partial vapor pressure in pore spaces $(P a)$ \\
\hline$\dot{q}_{w}$ & Power of the heating wire per unit length $(\mathrm{W} / \mathrm{m})$ \\
\hline$q^{\prime \prime \prime}$ & Volumetric heat generation rate $\left(\mathrm{W} / \mathrm{m}^{3}\right)$ \\
\hline$r$ & Radius $(m)$ \\
\hline$R_{w}$ & Specific gas constant for water vapor $(=461.5 \mathrm{~J} / \mathrm{kg} \cdot \mathrm{K})$ \\
\hline$S$ & Surface area $\left(m^{2}\right)$ \\
\hline$t$ & Time $(s)$ \\
\hline$u$ & Bulk velocity $(\mathrm{m} / \mathrm{s})$ \\
\hline
\end{tabular}




$$
\begin{array}{ll}
\forall & \text { Volume }\left(m^{3}\right) \\
T & \text { Temperature }\left(K \text { or }{ }^{\circ} C\right) \\
z & \text { Vertical distance }(m)
\end{array}
$$

\begin{tabular}{|c|c|}
\hline$\beta$ & Indicator for implicit/explicit iterations \\
\hline$\nabla$ & Del operator $\left(m^{-1}\right)$ \\
\hline$\varepsilon$ & Convergence criteria \\
\hline$\eta$ & Soil porosity ( $\mathrm{m}^{3}$ of pore space per $m^{3}$ of soil) \\
\hline$\kappa$ & Permeability of soil $\left(\mathrm{m}^{2}\right)$ \\
\hline$\mu$ & Dynamic viscosity $(\mathrm{kg} / \mathrm{m} \cdot \mathrm{s})$ \\
\hline$\varpi$ & Relaxation factor of iteration \\
\hline$\psi$ & Matric liquid (capillary) potential (pressure head) $(m)$ \\
\hline$\Phi$ & Total soil matric potential for liquid flow $(m)$ \\
\hline$\rho$ & Density $\left(\mathrm{kg} / \mathrm{m}^{3}\right)$ \\
\hline$\sigma_{w a}$ & Surface tension of water in air $(\mathrm{N} / \mathrm{m})$ \\
\hline$\tau$ & Empirical constant used in hydraulic conductivity equation \\
\hline$\theta$ & Volumetric moisture content/fraction ( $\mathrm{m}^{3}$ of water per $\mathrm{m}^{3}$ of soil) \\
\hline$\Theta$ & Degree of saturation \\
\hline$\varphi$ & Relative humidity \\
\hline$\zeta$ & Time-weighting function \\
\hline$\xi$ & Temperature gradient ratio $\left(=(\nabla T)_{p} /(\nabla T)\right)$ \\
\hline$\Omega$ & Domain of space discretized over a mesh of finite volumes \\
\hline$\Xi$ & Dummy variable that represents another/other variable(s) \\
\hline
\end{tabular}

\section{Greek symbols}

\section{Subscripts}

$\begin{array}{ll}a & \text { Air } \\ c & \text { Critical } \\ C V & \text { Pertaining to control volume }\end{array}$




$\begin{array}{ll}\text { eff } & \text { Effective } \\ l & \text { Liquid } \\ p & \text { Pore } \\ r e f & \text { Reference } \\ s & \text { Solid } \\ s a t & \text { Saturation } \\ s v & \text { Saturated vapor } \\ T & \text { Pertaining to thermal conditions } \\ \text { tot } & \text { Total } \\ \psi & \text { Pertaining to moisture conditions } \\ v & \text { Vapor } \\ w & \text { Water }\end{array}$

\section{Superscripts}

$T$

Transpose

(e) Pertaining to control volume $e$

$i$

Current iteration 


\section{ABBREVIATIONS}

FDM: finite difference method

FVM: Finite volume method

GSD: Grain size distribution

GTES: Ground thermal energy storage

HFM: Heat flux meter

ID: Inner diameter

LHS: Left hand side

OD: Outer diameter

PHCM: Pure heat conduction model

RH: Relative humidity

RHS: Right hand side

RMSE: Root mean square error

SR: Saturation ratio $=\forall_{w} / \forall_{\text {pore }}$

TCP: Thermal conductivity probe

TDMA: Tridiagonal matrix algorithm (or Thomas algorithm)

TES: Thermal energy storage

T-TDR: Thermo-time domain reflectometry 


\section{LIST OF TABLES}

Table 4.1. Tolerances and uncertainties of the components in the apparatus

Table 4.2. Basic physical characteristics of Canadian soils used in this thesis

Table 4.3. Experimental values and systematic uncertainties for $D_{T}, C, k$, and $\theta_{l}$ at $\approx 80^{\circ} \mathrm{C} \ldots .29$

Table 4.4. Experimental values and systematic uncertainties for $D_{T}, C$, $k$, and $\theta_{l}$ at $\approx 50^{\circ} \mathrm{C} \ldots 29$

Table 4.5. Experimental values and systematic uncertainties for $D_{T}, C$, $k$, and $\theta_{l}$ at $\approx 20^{\circ} \mathrm{C} \ldots 30$

Table 4.6. Highest standard deviations of the mean for $k$ and $C$ from 10 needles of 5 heat pulse probes 31

Table 4.7. Fitted coefficients of Eq. 4.8 for $k$ and $C$ of dry soils $\left(22^{\circ} \mathrm{C} \leq \mathrm{T} \leq 80^{\circ} \mathrm{C}\right)$ and the corresponding $\mathrm{R}^{2}$ values 32

Table 4.8. Measured thermal properties of wet soils $(\mathrm{SR} \approx 0.25$, room temperature) 36

Table 4.9. Measured thermal properties of wet soils ( $\mathrm{SR} \approx 0.50$, room temperature)...... 37

Table 4.10. Measured thermal properties of wet soils $(\mathrm{SR} \approx 0.50)$ as functions of temperature from $22^{\circ} \mathrm{C}$ up to the indicated highest temperature 38

Table 4.11. Fitted coefficients of Eq. 4.8 for $k$ and $C$ of a wet NB2 soil $\left(\mathrm{SR} \approx 0.50, \eta \approx 0.51,22^{\circ} \mathrm{C}\right.$ $\left.\leq \mathrm{T} \leq 90^{\circ} \mathrm{C}\right)$ 39

Table 4.12. Measured thermal properties of wet soils $(\mathrm{SR} \approx 0.70)$ 40

Table 4.13. Measured thermal properties of wet soils $(\mathrm{SR} \approx 0.70)$ as functions of temperature from $22^{\circ} \mathrm{C}$ to $90^{\circ} \mathrm{C}$ 40

Table 4.14. Times to reach $95 \%$ temperatures at steady-state conditions for the tested soils .... 42

Table 6.1. Thermal properties used in finite element simulations using COMSOL 60

Table 6.2. Major simulation parameters 68 
Table 7.1. Summary of worst \% errors (in terms of $T$ and $\theta$ ) of using Eq. 2.5 to simulate different soils under various heating conditions ............................................................................. 112

Table A1. Collision integral based on the Lennard-Jones potential ..................................... 120

Table A2. Physical and thermal properties of soil constituents ............................................ 122

Table A3. Estimated diameters and masses of sand, silt, and clay ....................................... 124 


\section{LIST OF FIGURES}

Figure 2.1. Example graph of soil moisture retention .................................................. 11

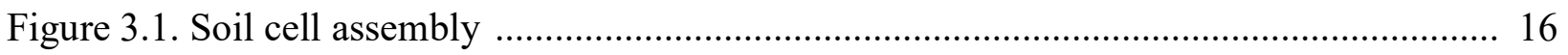

Figure 3.2. Soil cell with insulation ............................................................................. 18

Figure 3.3. An in-house made heat pulse probe ....................................................... 22

Figure 3.4. Summary of experimental methodology in this thesis ......................................... 24

Figure 4.1. A sample of temperature response of a temperature-sensing needle ................... 26

Figure 4.2. Thermal properties of dry QC2 soil $(\eta=0.42,0.45$ and 0.54$)$ with fitted linear relations

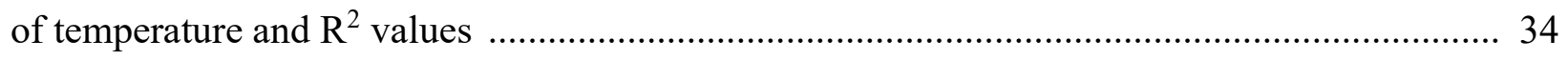

Figure 4.3. Thermal properties of soils ( $\mathrm{SR} \approx 0.25, T \approx 22^{\circ} \mathrm{C}$ ) with fitted linear relations of porosity

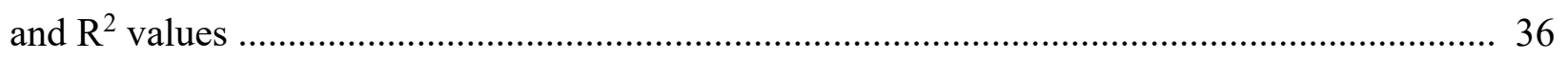

Figure 4.4. Thermal properties of NB2 soil $\left(\mathrm{SR}=0.25\right.$ and $0.50, T \approx 22^{\circ} \mathrm{C}$ ) with fitted linear

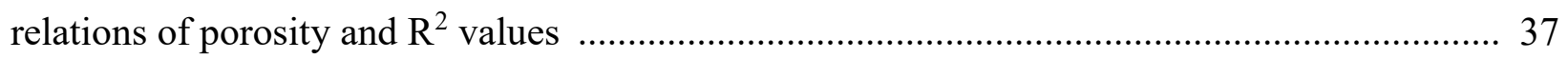

Figure 4.5. Fitted linear relations of thermal properties of NB2 soil ( $\mathrm{SR} \approx 0.50, \eta \approx 0.51)$ for three different soil samples and temperature ranges ................................................................ 39

Figure 4.6. Average thermal properties of tested soils at different temperatures ................... 41

Figure 5.1. One-dimensional discretization scheme ...................................................... 44

Figure 5.2. Control volume in axisymmetric geometry .............................................. 51

Figure 6.1. Geometry of the soil cell for finite element simulations using COMSOL ............ 61

Figure 6.2. Finest mesh of the finite element model using COMSOL ................................. 61

Figure 6.3. Simulated axial heat fluxes along the centerline of the soil column at different times for different number of elements in the finite element model using COMSOL ...................... 62 
Figure 6.4. Temperature distribution along the centerline of the soil column at different times for (a) $\mathrm{SR}=0.00$ and (b) $\mathrm{SR}=0.50$ 63

Figure 6.5. Simulated axial heat flux along the centerline of the soil column for (a) $\mathrm{SR}=0.00$ and (b) $\mathrm{SR}=0.50$ 64

Figure 6.6. Simulated isotherms and heat flow vectors at $t=6$ hours 64

Figure 6.7. Simulated heat fluxes along the height of the soil column at the temperature sensor location $(r=1.675 \mathrm{~cm})$ for (a) axial heat flux $(\mathrm{SR}=0)$, (b) radial heat flux $(\mathrm{SR}=0)$, (c) axial heat flux $(\mathrm{SR}=0.5)$, and $(\mathrm{d})$ radial heat flux $(\mathrm{SR}=0.5)$ 65

Figure 6.8. Radial soil temperature distribution $\left({ }^{\circ} \mathrm{C}\right)$ for $\mathrm{SR}=0.00$ in the middle of each section of the soil column from 15 minutes to 6 hours with time increment of 15 mins 66 Figure 6.9. COMSOL simulated temperature distributions along the stainless-steel tube wall of the soil column for (a) $\mathrm{SR}=0.50$ and (b) $\mathrm{SR}=0.00$ with time increment of one minute 67 Figure 6.10. Percentage RMS differences of $T$ and $\theta$ vs. number of nodes 70

Figure 6.11. Temperature distribution of semi-infinite soil column at different times using numerical and analytical solutions 71

Figure 6.12. Moisture distributions in Yolo light clay soil column from numerical and analytical solutions for (a) $\psi(t, 40 \mathrm{~cm})=0 \mathrm{~cm}$ and (b) $\psi(t, 40 \mathrm{~cm})=-25 \mathrm{~cm}$ 72

Figure 6.13. Simulated temperature at each needle (N\#) for the case of dry BC1 $(\eta=0.59)$ and vertical heating from top 74

Figure 6.14. Simulated temperature at each needle (N\#) for the case of dry $\operatorname{BC} 1(\eta=0.59)$ and vertical heating from bottom 75 Figure 6.15. Simulated temperature at each needle (N\#) for the case of dry BC1 $(\eta=0.59)$ and horizontal heating 76

Figure 6.16. Simulated (a) temperature and (b) moisture content responses at each needle for the case of wet $\mathrm{BC} 1(\eta=0.56, \mathrm{SR} \approx 0.25)$ and vertical heating from top 77 
Figure 6.17. Simulated (a) temperature and (b) moisture content responses at each needle for the case of wet $\mathrm{BC} 1(\eta=0.54, \mathrm{SR}=0.25)$ and vertical heating from bottom 78

Figure 6.18. Simulated (a) temperature and (b) moisture content responses at each needle for the case of wet $\mathrm{BC} 1(\eta=0.53, \mathrm{SR} \approx 0.25)$ and horizontal heating 80

Figure 6.19. Simulated (a) temperature and (b) moisture content responses at each needle for the case of wet $\mathrm{BC} 1(\eta=0.51, \mathrm{SR}=0.50)$ and vertical heating from top 82

Figure 6.20. Simulated (a) temperature and (b) moisture content responses at each needle for the case of wet $\mathrm{BC} 1(\eta=0.51, \mathrm{SR}=0.50)$ and vertical heating from bottom 83

Figure 6.21. Simulated (a) temperature and (b) moisture content at each needle for the case of wet $\mathrm{BC} 1(\eta=0.51, \mathrm{SR}=0.50)$ and horizontal heating 84

Figure 7.1. BC1 soil $(\eta=0.56, \mathrm{SR}=0.25)$ for the case of vertically heated from top: (a) measured heat fluxes and (b) \% errors in temperatures and heat fluxes 88

Figure 7.2. $\mathrm{BC} 1$ soil $(\eta=0.54, \mathrm{SR}=0.25)$ for the case of vertically heated from bottom: (a) measured heat fluxes and (b) \% errors in temperatures and heat fluxes 89

Figure 7.3. $\mathrm{BC} 1$ soil $(\eta=0.53, \mathrm{SR}=0.25)$ for the case of horizontally heated: (a) measured heat fluxes and (b) \% errors in temperatures and heat fluxes 90

Figure 7.4. $\mathrm{BC} 1$ soil $(\eta=0.51, \mathrm{SR}=0.50)$ for the case of vertically heated from top: (a) measured heat fluxes and (b) \% errors in temperatures and heat fluxes 90

Figure 7.5. BC1 soil $(\eta=0.51, \mathrm{SR}=0.50)$ for the case of vertically heated from bottom: (a) measured heat fluxes and (b) \% errors in temperatures and heat fluxes 91

Figure 7.6. $\mathrm{BC} 1$ soil $(\eta=0.51, \mathrm{SR}=0.50)$ for the case of horizontally heated: (a) measured heat fluxes and (b) \% errors in temperatures and heat fluxes 91 
Figure 7.7. NB2 soil ( $\eta=0.56, \mathrm{SR}=0.25)$ for the case of vertically heated from top: (a) measured heat fluxes and (b) \% errors in temperatures and heat fluxes

Figure 7.8. NB2 soil $(\eta=0.53, \mathrm{SR}=0.25)$ for the case of vertically heated from bottom: (a) measured heat fluxes and (b) \% errors in temperatures and heat fluxes 92

Figure 7.9. NB2 soil ( $\eta=0.53, \mathrm{SR}=0.25)$ for the case of horizontally heated: (a) measured heat fluxes and (b) \% errors in temperatures and heat fluxes 93

Figure 7.10. NB2 soil ( $\eta=0.55, \mathrm{SR}=0.50)$ for the case of vertically heated from top: (a) measured heat fluxes and (b) \% errors in temperatures and heat fluxes 93

Figure 7.11. NB2 soil $(\eta=0.53, \mathrm{SR}=0.50)$ for the case of vertically heated from bottom: (a) measured heat fluxes and (b) \% errors in temperatures and heat fluxes 93

Figure 7.12. NB2 soil ( $\eta=0.51, \mathrm{SR}=0.50)$ for the case of horizontally heated: (a) measured heat fluxes and (b) \% errors in temperatures and heat fluxes 94

Figure 7.13. QC2 soil ( $\eta=0.50, \mathrm{SR}=0.25)$ for the case of vertically heated from top: (a) measured heat fluxes and (b) \% errors in temperatures and heat fluxes 94

Figure 7.14. QC2 soil $(\eta=0.48, \mathrm{SR}=0.25)$ for the case of vertically heated from bottom: (a) measured heat fluxes and (b) \% errors in temperatures and heat fluxes 95

Figure 7.15. QC2 soil ( $\eta=0.47, \mathrm{SR}=0.25)$ for the case of horizontally heated: (a) measured heat fluxes and (b) \% errors in temperatures and heat fluxes 95

Figure 7.16. QC2 soil ( $\eta=0.44, \mathrm{SR}=0.50)$ for the case of vertically heated from top: (a) measured heat fluxes and (b) \% errors in temperatures and heat fluxes 95

Figure 7.17. QC2 soil ( $\eta=0.44, \mathrm{SR}=0.50)$ for the case of vertically heated from bottom: (a) measured heat fluxes and (b) \% errors in temperatures and heat fluxes 96 
Figure 7.18. Experimental results and comparisons of QC2 soil horizontally heated. SR $\sim 0.50$. (a): Measured heat fluxes. (b): \% error in temperatures and heat fluxes 96

Figure 7.19. (a) Measured moisture-content responses of $\mathrm{BC} 1(\eta=0.56, \mathrm{SR} \approx 0.50)$ at $42^{\circ} \mathrm{C}$ for the gravity test and (b) \% errors of simulated moisture contents 97

Figure 7.20. (a) Measured moisture-content responses of $\mathrm{BC} 1(\eta=0.55, \mathrm{SR} \approx 0.50)$ at $52^{\circ} \mathrm{C}$ for the gravity test and (b) \% errors of simulated moisture contents 98

Figure 7.21. (a) Measured moisture-content responses of $\mathrm{BC} 1(\eta=0.55, \mathrm{SR} \approx 0.50)$ at $62^{\circ} \mathrm{C}$ for the gravity test and (b) \% errors of simulated moisture contents 98

Figure 7.22. (a) Measured moisture-content responses of $\mathrm{NB} 2(\eta=0.51, \mathrm{SR} \approx 0.50)$ at $42^{\circ} \mathrm{C}$ for the gravity test and (b) \% errors of simulated moisture contents 99

Figure 7.23. (a) Measured moisture-content responses of NB2 $(\eta=0.51, \mathrm{SR} \approx 0.50)$ at $52^{\circ} \mathrm{C}$ for the gravity test and (b) \% errors of simulated moisture contents 100

Figure 7.24. (a) Measured moisture-content responses of $\mathrm{NB} 2(\eta=0.52, \mathrm{SR} \approx 0.50)$ at $62^{\circ} \mathrm{C}$ for the gravity test and (b) \% errors of simulated moisture contents 100

Figure 7.25. (a) Measured moisture-content responses of QC2 $(\eta=0.45, \mathrm{SR} \approx 0.50)$ at $42^{\circ} \mathrm{C}$ for the gravity test and (b) \% errors of simulated moisture contents 101

Figure 7.26. (a) Measured moisture-content responses of QC2 $(\eta=0.42, \mathrm{SR} \approx 0.50)$ at $52^{\circ} \mathrm{C}$ for the gravity test and (b) \% errors of simulated moisture contents 101

Figure 7.27. (a) Measured moisture-content responses of $\mathrm{QC} 2(\eta=0.42, \mathrm{SR} \approx 0.50)$ at $62^{\circ} \mathrm{C}$ for the gravity test and (b) \% errors of simulated moisture contents 102

Figure 7.28. (a) Measured moisture-content responses of $\mathrm{BC} 1$ (in horizontal position) with five layers of different initial SRs and (b) \% errors of simulated moisture contents 103 
Figure 7.29. (a) Measured moisture-content responses of NB2 (in horizontal position) with five different initial SRs and (b) \% errors of simulated moisture contents 104

Figure 7.30. (a) Measured moisture-content responses of QC2 (in horizontal position) with five layers of different initial SRs and (b) \% errors of simulated moisture contents 104

Figure 7.31. (a) Measured moisture-content responses of $\mathrm{BC} 1$ (in vertical position) with five layers of different initial SRs and (b) \% errors of simulated moisture contents 105

Figure 7.32. (a) Measured moisture-content responses of NB2 (in vertical position) with five different layers of initial SRs and (b) \% errors of simulated moisture contents 106

Figure 7.33. (a) Measured moisture-content responses of QC2 (in vertical position) with five layers of different initial SRs and (b) \% errors of simulated moisture contents 107

Figure 7.34. Percentage error of $T$ and $\theta$ (from Eq. 2.5 ) for $\mathrm{BC} 1$ soil $(\mathrm{SR} \approx 0.25)$ and vertically heated from top 108

Figure 7.35. Percentage error of $T$ and $\theta$ (from Eq. 2.5) for $\mathrm{BC} 1$ soil $(\mathrm{SR} \approx 0.25)$ and vertically heated from bottom 109

Figure 7.36. Percentage error of $T$ and $\theta$ (from Eq. 2.5 ) for $\mathrm{BC} 1$ soil $(\mathrm{SR} \approx 0.25$ ) and horizontally heated 109

Figure 7.37. Percentage error of $T$ and $\theta$ (from Eq. 2.5) for $\mathrm{BC} 1$ soil $(\mathrm{SR} \approx 0.50)$ and vertically heated from top 110

Figure 7.38. Percentage error of $T$ and $\theta$ (from Eq. 2.5) for $\mathrm{BC} 1$ soil (SR $\approx 0.50)$ and vertically heated from bottom 110

Figure 7.39. Percentage error of $T$ and $\theta$ (from Eq. 2.5) for $\mathrm{BC} 1$ soil ( $\mathrm{SR} \approx 0.50$ ) and horizontally heated 
Figure B1. Example of Matlab function

Figure C1. Design of heat pulse probe and its mold

Figure $\mathrm{C} 2$. Heating/cooling plate design

Figure C3. Design of soil column - Part I

Figure C4. Design of soil column - Part II

Figure C5. Design of outer container

Figure C6. Campbell Scientific digital system and DC power supply connected to the PC via 8 conductor cable from datalogger (CR-1000) 136

Figure C7. Lauda Proline RP1845 thermal baths 136

Figure E1. Simulated temperature at each needle $(\mathrm{N} \#)$ for the case of dry NB2 $(\eta=0.57)$ and vertical heating from top 141

Figure E2. Simulated temperature at each needle (N\#) for the case of dry NB2 $(\eta=0.57)$ and vertical heating from bottom. 141

Figure E3. Simulated temperature at each needle $(\mathrm{N} \#)$ for the case of dry NB2 $(\eta=0.57)$ horizontal heating 142

Figure E4. Simulated temperature and moisture content at each needle for the case of wet NB2 ( $\eta$ $=0.56, \mathrm{SR}=0.25)$ and vertical heating from top. $(\mathrm{a})$ : temperature response. $(\mathrm{b})$ : moisture content response

Figure E5. Simulated temperature and moisture content at each needle for the case of wet NB2 ( $\eta$ $=0.53, \mathrm{SR}=0.25)$ and vertical heating from bottom. (a): temperature response. (b): moisture content response 
Figure E6. Simulated temperature and moisture content at each needle for the case of wet NB2 ( $\eta$ $=0.53, \mathrm{SR}=0.25)$ and horizontal heating. (a): temperature response. (b): moisture content response

Figure E7. Simulated temperature and moisture content at each needle for the case of wet NB2 ( $\eta$ $=0.55, \mathrm{SR}=0.50)$ and vertical heating from top. $(\mathrm{a})$ : temperature response. $(\mathrm{b})$ : moisture content response

Figure E8. Simulated temperature and moisture content at each needle for the case of wet NB2 ( $\eta$ $=0.53, \mathrm{SR}=0.50)$ and vertical heating from bottom. (a): temperature response. $(\mathrm{b})$ : moisture content response.

Figure E9. Simulated temperature and moisture content at each needle for the case of wet NB2 ( $\eta$ $=0.51, \mathrm{SR}=0.50)$ and horizontal heating. $(\mathrm{a})$ : temperature response. $(\mathrm{b})$ : moisture content response 148

Figure E10. Simulated temperature at each needle $(\mathrm{N} \#)$ for the case of dry QC2 $(\eta=0.45)$ and vertical heating from top

Figure E11. Simulated temperature at each needle (N\#) for the case of dry QC2 $(\eta=0.45)$ and vertical heating from bottom

Figure E12. Simulated temperature at each needle $(\mathrm{N} \#)$ for the case of dry QC2 $(\eta=0.45)$ and horizontal heating 150

Figure E13. Simulated temperature and moisture content at each needle for the case of wet QC2 ( $\eta=0.50, \mathrm{SR}=0.25)$ and vertical heating from top. (a): temperature response. (b): moisture content response 151

Figure E14. Simulated temperature and moisture content at each needle for the case of wet QC2 ( $\eta=0.48, \mathrm{SR}=0.25)$ and vertical heating from bottom. (a): temperature response. $(\mathrm{b})$ : moisture content response 152

Figure E15. Simulated temperature and moisture content at each needle for the case of wet QC2 ( $\eta=0.47, \mathrm{SR}=0.25)$ and horizontal heating. (a): temperature response. (b): moisture content response 153 
Figure E16. Simulated temperature and moisture content at each needle for the case of wet QC2 $(\eta=0.44, \mathrm{SR}=0.50)$ and vertical heating from top. (a): temperature response. $(\mathrm{b})$ : moisture content response 154

Figure E17. Simulated temperature and moisture content at each needle for the case of wet QC2 ( $\eta=0.44, \mathrm{SR}=0.50)$ and vertical heating from bottom. (a): temperature response. (b): moisture content response. 155

Figure E18. Simulated temperature and moisture content at each needle for the case of wet QC2 ( $\eta=0.42, \mathrm{SR}=0.50)$ and horizontal heating. (a): temperature response. (b): moisture content response 156

Figure E19. Simulated temperature at each needle $(\mathrm{N} \#)$ for the case of dry soils and vertical heating from top (left part) and from bottom (right part) from 0 to 6 hour 157

Figure E20. Simulated temperature at each needle (N\#) for the case of dry soils and horizontal heating from 0 to 6 hour 158

Figure E21. Simulated temperature at each needle $(\mathrm{N \# )}$ for the case of wet soils $(\mathrm{SR} \sim 0.25)$ and vertical heating from top (left part) and from bottom (right part) from 0 to 6 hour 159

Figure E22. Simulated temperature at each needle $(\mathrm{N \# )}$ for the case of wet soils $(\mathrm{SR} \sim 0.50)$ and vertical heating from top (left part) and from bottom (right part) from 0 to 6 hour 160

Figure E23. Simulated temperature at each needle (N\#) for the case of wet soils being horizontally heated from 0 to 6 hour. $\mathrm{SR} \approx 0.25$ is on the left. $\mathrm{SR} \approx 0.50$ is on the right 161

Figure E24. Simulated moisture content at each needle $(\mathrm{N} \#)$ for the case of wet soils $(\mathrm{SR} \sim 0.25)$ and vertical heating from top (left part) and from bottom (right part) from 0 to 18 hour 162

Figure E25. Simulated moisture content at each needle $(\mathrm{N} \#)$ for the case of wet soils $(\mathrm{SR} \sim 0.50)$ and vertical heating from top (left part) and from bottom (right part) from 0 to 18 hour 163 
Figure E26. Simulated heat flux and \% error at top and bottom HFMs of wet soils $(\mathrm{SR} \approx 0.25)$ and vertical heating from top

Figure E27. Simulated heat flux and \% error at top and bottom HFMs of wet soils $(\mathrm{SR} \approx 0.25)$ and vertical heating from bottom 165

Figure E28. Simulated heat flux and \% error at top and bottom HFMs of wet soils $(\mathrm{SR} \approx 0.25)$ and horizontal heating 166

Figure E29. Simulated heat flux and \% error at top and bottom HFMs of wet soils $(\mathrm{SR} \approx 0.50)$ and vertical heating from top 167

Figure E30. Simulated heat flux and \% error at top and bottom HFMs of wet soils $(\mathrm{SR} \approx 0.50)$ and vertical heating from bottom 168

Figure E31. Simulated heat flux and \% error at top and bottom HFMs of wet soils $(\mathrm{SR} \approx 0.50)$ and horizontal heating 169

Figure E32. Centerline radial heat flux (for dry Matilda) from COMSOL using different number of elements 170

Figure F1. Sample of soil preparation before compaction into the soil column 171

Figure F2. Sample of soil compaction 172

Figure F3. Sample of soil removal after an experiment 173

Figure F4. Setup for calibrations of probes which are immersed in a solution with 1\% agar and $99 \%$ water 174 


\section{CHAPTER 1 - INTRODUCTION}

\section{1 - General Review}

Global warming is currently causing serious issues around the globe. The super typhoon Haiyan landed and devastated the Philippines on Nov. 8, 2013 with its extra-ordinary intensity of $170 \mathrm{kts}$ (about $315 \mathrm{~km} / \mathrm{h}$ ) which is well above the threshold of $135 \mathrm{kts}$ of the existing highest category 5. Lin et al. [1] explained that the super typhoon happened as a result of the warming on the west and cooling on the east of the Pacific Ocean. In addition, simulation results of Boer [2] showed that the ocean, on average, warms the land, resulting in harsher living conditions to most humans and increasing the mortality, especially older people and those with cardiovascular diseases [3]. Approximately 22,000 to 45,000 heart-related deaths occurred across Europe over two days in August 2003 [4], [5] with $3.5^{\circ} \mathrm{C}$ above normal [6]. The spread and transmission rates of vector-borne infections also increase with rising temperature by faster pathogen maturation and replication within mosquitoes and denser insect population in a particular area (Costello et al. [7]). Consequently, malaria, tick-borne encephalitis, dengue fever, and many other infectious diseases will become more widespread with climate warming [8].

Moreover, global warming can severely damage the natural habitat. Rising temperature has negative impacts on aviation species by changing their reproductive performances (Penteriani et al. [9]). Habel et al. [10] sampled 203 individuals of Lycaena Helle butterfly and found that climate warming decreases their connectivity and increases their individual extinction risk. Consequently, many plants and trees will have fewer fertilized seeds when the butterfly population decreases. Ramírez-Amezcua et al. [11] modeled the existence alpine ecosystem by obtaining 21 climatic and topographic variables from field trips to the Trans-Mexican Volcanic Belt. They estimated that by $2070,58 \%$ of the current alpine ecosystem will disappear due to global warming.

Therefore, many governments around the world have devoted heavy efforts in reducing global warming which results from heavy consumption of energies (mostly fossil fuels due to their relative inexpensiveness) ([12], [13]). More uses of renewable energies have been encouraged with 
the introduction and implementation of rebates and incentives. A highly abundant and attractive renewable energy is the solar radiation from the Sun.

In order to store the thermal energy from solar radiation, thermal energy storages (TESs) are needed. A common and widely used TES form is the ground thermal energy storage (GTES). To better utilize the solar energy, it is beneficial to store the solar heat in high-temperature ground because the heat can be readily retrieved for spacing heating without the use of a heat pump to upgrade the heat first. A first-in-the-world example of successful application of using hightemperature GTES is the Drake Landing Solar Community in Okotoks, Alberta, Canada. The community collects and stores the solar thermal energy in the ground at high temperatures of up to $80^{\circ} \mathrm{C}$ over the summer season for the community's $90 \%$ space heating needs in the winter. The Okotoks ground in Alberta contains mostly clay which has low moisture diffusivity, so the GTES of the Drake Landing Solar Community can store and retain most of the heat in the summer. However, not everywhere in the world has such type of soil. Therefore, understanding how hightemperature heat and moisture transfer in various soil types is beneficial to the design of hightemperature GTES.

Generally, soil is considered as a non-homogenous and non-isotropic porous material. The term soil, as used by engineers, refers to a complicated material consisting of solid particles of various compositions (e.g., minerals and organics) and various shapes and sizes that are randomly arranged with pore spaces between them. The pores contain air, water in its various phases (vapor, liquid, and/or ice), and sometimes other liquidous or gaseous contaminants. The composition of naturally occurring soil changes because of geographical variations. These changes are from the weather and the continuously varying temperature field where the soil is. On the surface of the soil, there is seasonal daily temperature variation due to weather. Underneath the surface, the hot core of the Earth keeps providing heat to the relatively cool crust of the Earth on which buildings are constructed. Together with the weights from the Earth's surface, these changing thermal gradients alter the soil composition, particularly the changes in the amount, phase, and condition of water.

Therefore, understanding the moisture and heat distribution in soil is useful in many applications such as agriculture, building science, underground systems (e.g., gas pipes and electrical cables), and designs of GTESs. 


\section{2 - Background and Motivation}

The temperature and moisture fields are usually coupled. Thermal gradients cause moisture (in both the vapor and liquid phases) to move from hotter to cooler areas, which simultaneously affects the thermal gradients. Thermal gradients and the associated moisture transfer (which is majorly considered as a molecular diffusion process) cause changes in the moisture content and pressure inside the soil. In unsaturated soils, decreasing moisture content comes with a drop in the thermal liquid moisture flow but with a rise in the thermal vapor moisture flow [14].

To predict heat transfer (steady or transient) in soil requires the knowledge of the soil's thermal properties such as thermal conductivity and heat capacity. While heat conduction is the dominating mechanism, other mechanisms are possible. Generally, convection and radiation have negligible effects [15]. In addition, the heat transfer process may be affected by water phase changes in the soil. Freezing of water or melting of ice within the soil may also result in significant latent heat effects.

The dominant heat conduction occurs in all soil constituents. While heat conduction is passed by vibration in solid particles, it operates in air or water liquid/vapor through molecular collisions and a consequent increase in the molecules' mean kinetic energy. The heat conducted in soil increases with higher soil dry bulk density $\rho_{d b}\left(\right.$ i.e., $\left.\rho_{d b}=\rho_{s} / \forall_{t o t}\right)$ and higher degree of saturation $S R\left(\right.$ i.e., $\left.S R=\forall_{w} / \forall_{\text {pore }}\right)$ and passes through all available paths with the main path being contacting solids. Thermal contact resistance can also exist to block the heat conduction and creates a sudden discontinuity in the soil temperature at the contact between the solid particles and the interstitial liquid or other solid particles [16].

Another heat transfer mechanism in soil is convection. There are two types of convection: free and forced. Free convection of a fluid is a mass and heat transport phenomenon as a result of temperature gradient in a gravity field. It occurs as its density changes in response to the temperature changes. Meanwhile, forced convection happens when the air or water is forced to move through the pores of the soil or rock by pressure differences. The convection effects are usually small but may enhance heat transfer by $20 \%$ in very coarse soils in comparison to the clay soils [17]. As a result, heat and mass transfer in soils have been an important research area in the past decades. 
The mathematical analysis of soil response under atmospheric conditions is problematic since the thermal properties of the soil strongly depend on the temperature and moisture content which are difficult to measure accurately. The pioneers in modeling coupled heat and mass transfer in porous media include Philip and de Vries [18] and Luikov [19]. Theoretical expressions for the thermal moisture and isothermal moisture diffusivities were developed together with two governing partial differential equations of heat and moisture transfer which are dependent on soil's

hydraulic conductivity, temperature gradients, moisture potential, and volumetric water content. Heat conduction incorporating latent heat transfer by water vapor diffusion was considered and generalized with moisture and latent heat storage in vapor phase and sensible heat transfer by liquid migration in the soil. As a result, many mathematical models focus on modifying the approaches of Philip's, de Vries', and Luikov's [20].

The heat and mass transfer theories have been applied to many areas such as underground cables and pipelines and GTES. About $40 \%$ of all greenhouse gas emissions in developed countries are originated from building equipment, approximately $60 \%$ of which is produced by heating and cooling systems [21], [22]. As a result, extensive studies have focused on improving the efficiencies of the heating and cooling systems. One method to improve the efficiencies is to utilize solar radiation and GTES system in heating-dominated areas [23], [24], [25], [26]. In other words, radiation from the Sun is stored for future heating requirements of the building in an area with a cold climate (e.g., Canada). Nonetheless, it is expensive to build such thermal storage systems. As a result, the heat and moisture transfer in the ground is important for properly studying and designing solar-assisted GTESs which may be required to operate from $40^{\circ} \mathrm{C}$ to $95^{\circ} \mathrm{C}$. Under this high temperature condition, the air and moisture contents in the soil become highly energetic and tend to migrate to colder regions in the soil. Consequently, heat is also carried with the migrations (caused by thermal gradient) in the soil.

\section{3 - Research Objectives}

To the author's best knowledge, there is no comprehensive experiment performed under hightemperature conditions (above $40^{\circ} \mathrm{C}$ ) although theoretical models of heat and moisture transfer have been developed. Hedayati-Dezfooli [27] designed and built an experimental apparatus to 
study the heat and moisture transfer in soils in the lab. He also developed an experimental methodology to test soils from $40^{\circ} \mathrm{C}$ to $90^{\circ} \mathrm{C}$. However, Hedayati-Dezfooli's apparatus was limited to collecting data for up to three hours and his methodology contained some over-simplifying assumptions to calculate the soil's porosity and heat capacity. As a result, the objectives of this thesis are:

A. To improve an existing experimental methodology and apparatus, by Hedayati-Dezfooli [27], to better study the heat and moisture transfer in soils at temperatures above $40^{\circ} \mathrm{C}$.

B. To verify and calibrate (if necessary) a theoretical model of heat and moisture transfer in soils developed by Deru [28].

C. To provide experimental techniques to test three different soil types with different textures under various conditions at temperatures above $40^{\circ} \mathrm{C}$.

\section{4 - Thesis Organization}

The main purposes of the following chapters are:

- Chapter 2 gives a literature review on the theory of heat and mass transfer in soils and shows the governing equations for coupled heat and mass transfer in soils which will be used in the present study.

- Chapter 3 describes the experimental procedures and techniques that will be used to obtain experimental data for verifying the theoretical model.

- Chapter 4 shows and discusses the experimental results and their experimental uncertainties.

- Chapter 5 presents the development of the one-dimensional and axisymmetric numerical formulations using the finite volume method (FVM).

- Chapter 6 examines the heat transfer characteristics in the experimental soil column using COMSOL for justifying the applicability of the one-dimensional formulations. The computer code of the numerical solution is verified with analytical solutions. In addition, samples of numerical simulations of differential heating from the experiments are illustrated. 
- Chapter 7 compares the pure heat conduction model with the experimental results and verifies the theoretical model of coupled heat and mass transfer in soils.

- Chapter 8 concludes the thesis and provides further ideas and recommendations for future works. 


\section{CHAPTER 2 - BACKGROUND AND LITERATURE REVIEW}

\section{1 - Introduction}

Soil is a complicated material consisting of solid particles of various compositions (e.g., minerals and organic materials) and different shapes and sizes that are randomly arranged with pore spaces (which usually contain air and water) in between. With the moisture and heat transfers, the thermal properties of the soil are affected [29]. As shown by Nikolaev [30], the thermal conductivity of the soil is highly dependent on the moisture (or water) content and temperature (especially for $T>40^{\circ} \mathrm{C}$ ) of the soil and generally increases with higher moisture content and temperature. Consequently, a good and thorough comprehension of heat and moisture transport phenomena (especially the coupled heat and moisture movements) in the soil can enable better and more realistic underground simulations.

\section{2 - Literature Review on the Theory of Heat and Mass Transfer}

Modelling transport phenomena in the ground has been of great interests. Early investigators discovered that there were water movements in soil materials subjected to a thermal gradient (Gurr et al. [31] and Taylor and Cavazza [32]). However, it was observed that the apparent vapor diffusion coefficient was 10 times higher than expected on the basis of simple diffusion laws, so several researchers attempted to explain the observation (Philip and de Vries [18] and Derjaguin and Melnikova [33]). As a result, it was found that the transport phenomena in soils could be successfully studied if heat and moisture movements in soils are considered together (Taylor and Cary [34]).

One early work was done by Dempsey [35] who studied the coupled heat and moisture transfer and applied the finite difference method to numerically study the one-dimensional moisture transfer in Lakeland fine sand (AASHO Classification A-3) under isothermal conditions. He then compared the numerical results with laboratory experimental data. The comparisons showed good 
agreements between the numerical work and the lab data. The maximum temperature considered was under $25^{\circ} \mathrm{C}$.

In 1986, Shen [36] calculated the heat and moisture transfer in soil near a basement wall using fully coupled equations. The calculations were based on a sand and a loam soil for two 15-day periods, one in the winter and one in the summer. It was found that using uncoupled equations could lead to notably differences in the temperature and moisture content regimes.

In 2001, Rees et al. [37] explored the effects of the water table on the heat loss from ground contacting structures using coupled heat and moisture transfer equations. Very simple heat and moisture balances on surfaces were applied. Moisture gradient was not applied but was considered to be in gravitational equilibrium. Although having limited results, Rees et al. showed that the soil moisture has significant effects in buildings' heat losses. To aid the limited results by Rees et al., Janssen et al. [38] applied fully coupled equations of heat and moisture transfer to further study the influence of soil moisture in the unsaturated zone. Janssen et al. indicated that the coupled calculations give significantly higher heat losses. In addition, the latent heat transfer by thermal vapor diffusion has a notable influence on the foundation heat loss.

In a paper by Hornet et al. [39], the transient basement heat loss to the ground was numerically investigated using a 2D finite element ANSYS 14.0 software package. Many aspects of the ground and weather were considered such as solar radiation, precipitation, and heat and mass transfer. Total daily heat losses for 365 days were simulated and were used to estimate the thermal conductivity of the ground by comparing the heat-and-moisture-transfer model with the simpleheat-conduction one. In other words, an effective thermal conductivity was applied in each model to produce a set of simulated results and was varied until the two sets of simulated results closely matched each other. Experimental work was not done to verify the simulated results.

Marshall and Fuhrmann [40] studied how rainfall could affect underground electric cables under steady-state and transient conditions. Coupled heat and moisture transfer equations were used with finite difference method. It was shown that under steady-state computations, the temperature-related degradation of the cable was over-estimated while the moisture-related degradation of the cable was under-estimated. The reason was the surface temperature of and the moisture content around the cable were, respectively, lower and higher than those from the 
transient conditions. Consequently, care should be taken which conditions are more appropriate to simulate in the current project.

Since the moisture content is an important factor in determining the soil's thermal properties, Topp et al. [41] showed that the water content in soils can be reliably measured using the timedomain reflectometry (TDR) method. The water content $\left(\theta_{w}\right)$ of soil is the main factor influencing the soil's dielectric constant which is related to the velocity of the electromagnetic pulse waves transmitted in the soil. As a result, the measured pulse waves from the TDR method can be used to determine the soil moisture content.

In 1999, Ren et al. [42] combined the heat pulse method and TDR technique into one thermotime domain reflectometry (T-TDR) probe which can simultaneously measure soil volumetric water content, thermal conductivity $(k)$, volumetric heat capacity $\left(\rho C_{p}\right)$, and thermal diffusivity $\left(D_{T}\right)$. Thermal property measurements on agar-water showed high accuracy (about $2 \%$ ) with the T-TDR probe. In 2005, Horton et al. [43] conducted measurements of soils with different bulk densities and water contents at the regulated temperature of $20.3^{\circ} \mathrm{C}$. The clay contents of the soil samples ranged from $11.6 \%$ to $36.7 \%$. From eight different soil studies, the heat pulse and TDR methods showed reliable results for the water content measurements with root mean square errors (RMSEs) of $0.022 \mathrm{~m}^{3} / \mathrm{m}^{3}$ and $0.023 \mathrm{~m}^{3} / \mathrm{m}^{3}$, respectively. Heitman et al. [44] developed a closed soil cell that provides one-dimensional conditions and allows in-situ measurements of temperature and thermal conductivity under momentary boundary conditions. The soil cell comprised of a small tube (ID $89 \mathrm{~mm} \times$ length $100 \mathrm{~mm}$ ) and a larger tube (ID $209 \mathrm{~mm} \times$ length $100 \mathrm{~mm}$ ). The transient temperature, water content, and thermal conductivity distributions in the soil cell were measured using the heat pulse and TDR methods. Four different soil types were tested under temperatures ranging from 15 to $40^{\circ} \mathrm{C}$.

Recently, Zhang et al. [45] studied the application of a new T-TDR probe in measuring the thermal properties, moisture content, and dry density of sand-kaolin clay mixtures with clay content ranging from $0 \%$ to $30 \%$ by dry weight in their laboratory. The results from Zhang et al. showed that the T-TDR probe can be successfully and accurately used to measure the thermal properties of sand-kaolin clay mixtures. It was found that the thermal conductivity can be more accurately obtained than the thermal diffusivity and volumetric heat capacity can be. 
Innovation in efficient energy usage has led to practical examples of using high-temperature GTESs in the world such as the Drake Landing Solar Community (DLSC) in Okotos, Alberta, Canada. Over $90 \%$ of the space heating requirements of 52 detached houses in DLSC are supplied by solar energy through a district heating and GTES system [46]. A borehole thermal energy storage (BTES) field, which can be up to $80^{\circ} \mathrm{C}$, is used to store the summer heat for use in the following winter. TRNSYS and ESP-r were used at the design phase of the DLSC to estimate the BTES efficiency and agreed well with the measured data. However, Sibbit et al. [47] indicated that more investigations could be carried out to lower the costs and improve performance of future systems of similar or much larger sizes.

Another practical example of using seasonal GTESs (up to $80^{\circ} \mathrm{C}$ ) with solar assistance is the SOLARTHERMIE-2000 Program in Germany [48], a pilot plan in an effort to reduce carbon footprints. The performance of the system was found to be in good agreements with initial design simulations. Other economical aspects were also studied. Other practical examples of GTESs are the seasonal GTES (up to $72^{\circ} \mathrm{C}$ ) in Thueringen, Germany [49] and a pilot plant for testing hightemperature GTES (up to $90^{\circ} \mathrm{C}$ ) in Linkoping, Sweden [50].

Although the practical examples show good agreements between measurements and initial design simulations, the GTESs are in clay ground with low water permeability and small moisture movement effects. The soil conditions in other places may not be so ideal. For more permeable soils such as sand, moisture movement (particularly caused by thermal gradient) significantly influences thermal conductivity and heat capacity, resulting in poor performance [49]. In addition, searches through the literature showed very few works on high-temperature heat and moisture transfer with experimental or field comparisons. For example, the works mentioned prior to the practical examples of GTESs in this section either only showed numerical simulations or compare numerical results with experimental data of $35^{\circ} \mathrm{C}$ and below. Therefore, high-temperature $(T>$ $40^{\circ} \mathrm{C}$ ) heat and moisture transfer with experimental data in various ground conditions is still in early stages. 


\section{3 - Governing Equations for Coupled Heat and Moisture Transfer in Soils}

The total soil water potential $\Phi$ (an indication of how easily moisture can move through the soil) can be assumed as:

$$
\Phi=\psi+z
$$

where $z$ is the gravitational potential of the soil (taken as positive upwards) and $\psi$ is the matric potential of the soil. It is the result of the capillary and adsorptive forces between water and the soil matrix. $\psi$ is defined as the potential energy of pure water (without external forces) at a reference pressure (atmospheric), reference temperature, and reference elevation of zero.

The relationship between the matric potential and volumetric moisture content of the soil can be observed from the soil moisture retention curve which is shown as an example in Fig. 2.1. For example, when the volumetric moisture content for loamy sand is $0.140 \mathrm{~m}^{3} / \mathrm{m}^{3}$, its matric potential is $-1.0 \mathrm{~m}$. When the moisture content for loamy sand is $0.395 \mathrm{~m}^{3} / \mathrm{m}^{3}$, its matric potential is almost $0 \mathrm{~m}$. When the moisture content for loamy sand is $0.005 \mathrm{~m}^{3} / \mathrm{m}^{3}$, its matric potential is almost $-10^{6}$ $m$. The moisture content of the soil can be calculated using the matric potential with Eq. A.10 in the Appendix A.2. The flatness of the loamy sand shows that the moisture drains quicker and the steeper slope of the clay indicates that the moisture in the clay does not drain as quickly because clay holds water better than sands do.

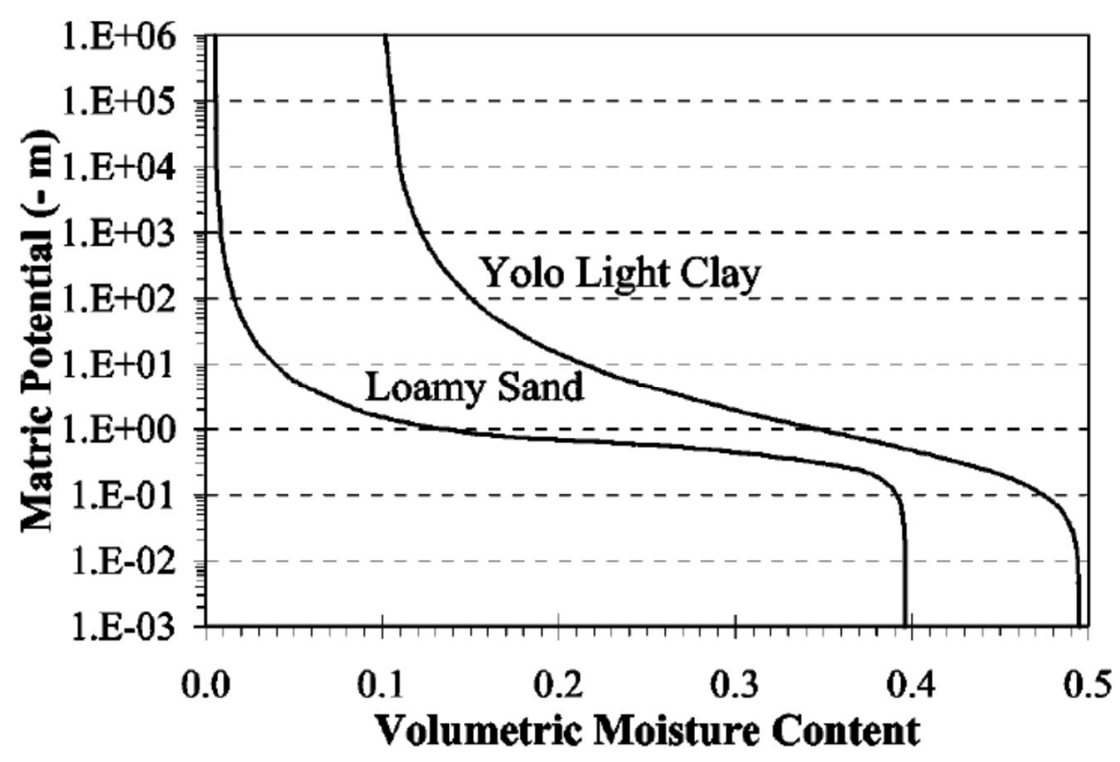

Figure 2.1. Example graph of soil moisture retention [28]. 
The heat and mass transfer equations can be formulated based on physical processes that occur in the soil as described by Deru [28]. The main simplifying assumptions made in the derivations of the governing equations are that the soil is homogeneous and isotropic within each defined unit of soil. Darcy's law can be extended to unsaturated soil as:

$$
\boldsymbol{u}_{l}=-K \nabla \Phi
$$

where $\boldsymbol{u}_{l}$ is the speed of the liquid flow in the soil, $K$ is the hydraulic conductivity of the soil $\left(K=\kappa \rho_{w} g / \mu_{w}\right), \nabla \Phi$ is the gradient vector of total soil water potential $\left(\nabla \Phi=\nabla P / \rho_{w} g\right), \kappa$ is the permeability of the soil, $\nabla P$ is the pressure gradient vector, and $\rho_{w}$ and $\mu_{w}$ are the density and dynamic viscosity of water, respectively.

Substitution of Eq. 2.1 into Eq. 2.2 yields:

$$
\boldsymbol{u}_{l}=-K \nabla \psi-K \hat{k}
$$

Applying continuity equation to the liquid moisture content of a control volume (CV) of soil, the conservation of liquid can be written as:

$$
\frac{\partial \theta_{l}}{\partial t}=-\nabla \cdot \boldsymbol{u}_{l}-E
$$

where $E$ is the evaporation rate $\left(s^{-1}\right)$.

The following further assumptions can be made:

- The vapor in the soil behaves as an ideal gas.

- A gas-filled pore has a uniform and constant total pressure $P$.

- The liquid and vapor in the soil are in thermodynamic equilibrium.

- There is no solute.

- The heat capacity of the air in the soil is neglected as it is much lower than those of the moisture (if any) and the solid.

Applying the further assumptions, the governing equations of heat and moisture transfer in soils are [28]: 


$$
\begin{gathered}
C_{\psi m} \frac{\partial \psi}{\partial t}+C_{T m} \frac{\partial T}{\partial t}=\nabla \cdot\left(D_{\psi m} \nabla \psi\right)+\nabla \cdot\left(D_{T m} \nabla T\right)+\frac{\partial K}{\partial z} \\
C_{\psi T} \frac{\partial \psi}{\partial t}+C_{T T} \frac{\partial T}{\partial t}=\nabla \cdot\left(D_{\psi T} \nabla \psi\right)+\nabla \cdot\left(k_{e f f} \nabla T\right)+C_{p w} \dot{\boldsymbol{m}}_{w} \cdot \nabla T
\end{gathered}
$$

where $C_{\psi m}=C_{\psi l}+C_{\psi v}, \quad C_{T m}=C_{T l}+C_{T \nu}, \quad C_{\psi T}=\rho_{l} \cdot h_{f g} \cdot C_{\psi v}, \quad C_{T T}=C_{s o i l}+\rho_{l} \cdot h_{f g} \cdot C_{T v}$,

$$
\begin{aligned}
& C_{\psi l}=\left(\frac{\partial \theta_{l}}{\partial \psi}\right)_{T}, \quad C_{T l}=\left(\frac{\partial \theta_{l}}{\partial T}\right)_{\psi}, \quad D_{\psi m}=K+D_{\psi v}, \quad D_{T m}=D_{T v}, \quad D_{\psi T}=\rho_{l} \cdot h_{f g} \cdot D_{\psi v}, \\
& D_{\psi v}=f\left(\theta_{l}\right) D_{v a} \frac{\rho_{v s}}{\rho_{l}} \frac{\varphi g}{R_{w} T}, \quad \dot{m}_{w}=\rho_{l} u_{l}, \\
& C_{\psi v}=\frac{\rho_{v s} \varphi}{\rho_{l}}\left[\frac{\left(\eta-\theta_{l}\right) g}{R_{w} T}-\left(\frac{\partial \theta_{l}}{\partial \psi}\right)_{T}\right], \quad C_{T v}=\frac{\rho_{v s} \varphi}{\rho_{l}}\left[\frac{\eta-\theta_{l}}{\rho_{v s}}\left(\frac{\partial \rho_{v s}}{\partial T}\right)-\left(\frac{\partial \theta_{l}}{\partial T}\right)_{\psi}\right], \\
& D_{T v}=f\left(\theta_{l}\right) D_{v a} \varphi \frac{\rho_{v s}}{\rho_{l}}\left[\frac{1}{\rho_{v s}} \frac{\partial \rho_{v s}}{\partial T}-\frac{\psi g}{R_{w} T^{2}}+\frac{g}{R_{w} T}\left(\frac{\partial \psi}{\partial T}\right)_{\theta}\right] \frac{(\nabla T)_{p}}{\nabla T}, \quad \varphi=\exp \left(\frac{\psi g}{R_{w} T}\right) \\
& K=K_{s a t} \frac{(\nabla T)_{p}}{\nabla T}=\xi \cdot\left(\theta_{w}+\xi_{p} \theta_{p}+\sum_{i=1}^{n} \xi_{i} \theta_{i}\right)^{-1}
\end{aligned}
$$

$\alpha, n, m(=1-(1 / n))$ are fitting parameters for the water retention curve proposed by van Genuchten [51] and [52]. $R_{w}$ is the gas constant for water vapor $=461.5 \mathrm{~J} /(\mathrm{kg} \cdot \mathrm{K}) . f\left(\theta_{l}\right)$ is the vapor diffusion correction factor: $f\left(\theta_{l}\right)=\left[\begin{array}{ll}\eta, & \theta_{l} \leq \theta_{k} \\ \left(\eta-\theta_{l}\right)+\left(\eta-\theta_{l}\right) \theta_{l} /\left(\eta-\theta_{k}\right), & \theta_{l}>\theta_{k}\end{array}\right.$.

$\theta_{k}$ is the critical moisture content below which the hydraulic conductivity falls to a value much lower than its value at saturation. de Vries [53] defined $\theta_{k}$ as $0.03 \mathrm{~m}^{3} / \mathrm{m}^{3}$ for coarsetextured soils, $0.04 \mathrm{~m}^{3} / \mathrm{m}^{3}$ for medium-textured, and $0.075 \mathrm{~m}^{3} / \mathrm{m}^{3}$ for fine-textured. $k_{\text {eff }}$ is the effective thermal conductivity of the soil which includes effective heat conduction by thermally driven vapor diffusion. A model for $k_{\text {eff }}$ is described in Appendix A.5.

More definitions of other parameters can be found in Appendix A.

Eqs. 2.5a is the moisture transfer equation in the soil and Eq. 2.5b is the energy transfer equation in the soil. The terms on the left hand sides correspond to the stored mass and energy due to the temporal change in matric potential and temperature. The first two terms on right hand sides 
account for the mass transfer (Eq. 2.5a) and heat transfer (Eq. 2.5b) respectively due to moisture and temperature gradients. The last term in Eq. 2.5a represents the mass transfer by gravitational effects while that in Eq. $2.5 \mathrm{~b}$ is the sensible heat transfer by bulk liquid flow. The second term in square brackets of $D_{T v}$ is significant for $\psi<-10^{-4} m$ while the third term is significant for $\psi<$ $-10^{-5} m[28]$.

As indicated by Deru [28], the last term on the RHS of Eq. $2.5 \mathrm{~b}$ is significant for only a short period after a large influx of moisture such as rainfall or irrigation. In this study, because there is no moisture addition to the soil cell during experimentation, the last term on the RHS of Eq. $2.5 \mathrm{~b}$ is ignored.

\section{4-Summary}

In this chapter, the theory of heat and mass transfer is briefly introduced. Due to the complexity of the soil system, simple heat conduction cannot be applied when modelling the heat and mass transfer in the soil system, especially at high-temperature conditions. The transfer is a coupled phenomenon where the temperature and the matric potential (or the moisture content) in the soil depend on each other, affecting the thermal properties of the soil and rendering the simple heat conduction model inaccurate.

Numerous researchers have studied the heat and mass transfer in the ground so that better engineering designs and applications (e.g., GTES and underground cables and pipes) can be made. The studies range from pure simulations to field works. Nonetheless, most studies were performed at temperatures below $40^{\circ} \mathrm{C}$.

Deru [28] developed a theoretical model (Eq. 2.5) to describe the coupled heat and mass transfer in the ground. He verified the model using field data and the finite element method for the numerical solution. However, the data were taken at temperatures below $40^{\circ} \mathrm{C}$ which is often not the case for high-temperature applications such as the high-temperature GTES up to $90^{\circ} \mathrm{C}$.

Therefore, it is the objective of this thesis to explore heat and mass transfer in soils under high-temperature conditions $\left(40^{\circ} \mathrm{C}\right.$ or more). In the next chapter, experimental apparatus will be 
shown and experimental techniques are described. Since there will be many cases that will be shown later in the simulated results, readers are advised to familiarize how the tests are run. 


\section{CHAPTER 3 - EXPERIMENTAL APPARATUS AND METHODOLOGY}

\section{1 - Experimental Apparatus}

The experimental soil cell designed and built by Hedayati-Dezfooli [27] is utilized in the present study of heat and moisture transfer in soils. Fig. 3.1 shows the overall assembly of the soil cell. For ease of viewing, the outer stainless-steel tube and lids of the soil column are shown transparently. Water hose connectors are connected to thermally-controlled water baths (which provide the heating and cooling to the aluminum plates) through water hoses. The soil column is a stainless-steel tube (63.5 $\mathrm{mm}$ ID, $76.2 \mathrm{~mm}$ OD, $147.9 \mathrm{~mm}$ length) that has five slots for inserting five heat pulse probes. Refer to Appendix $\mathrm{C}$ for more details about the dimensions of the components in the soil cell.

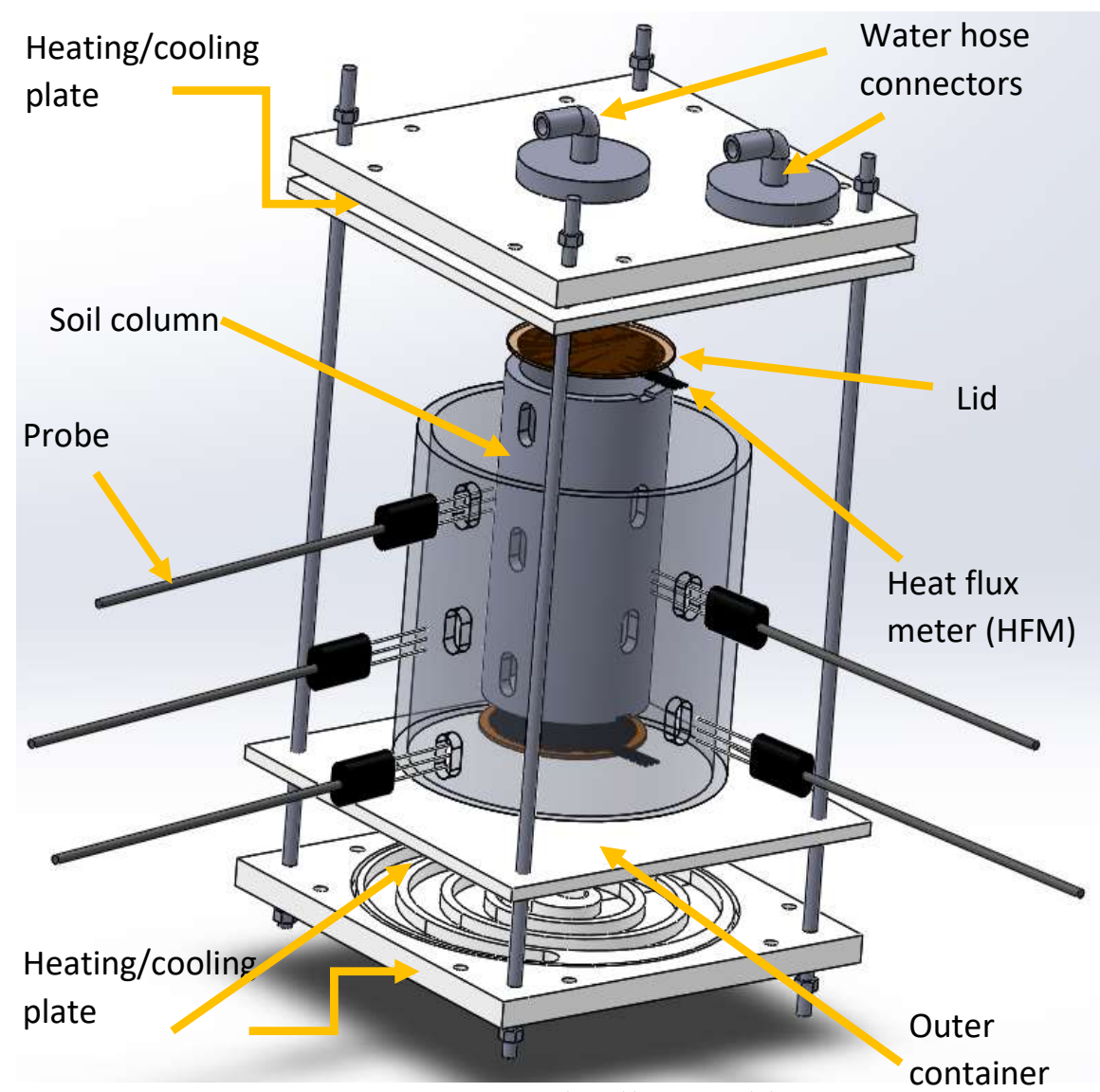

Figure 3.1. Soil cell assembly. 
The soil cell in Fig. 3.1 is connected to a control system that collects experimental data and has the following components:

1. Data acquisition (DAQ) system: This system is composed of a datalogger (CR1000), a thermocouple multiplexer (AM25T), and a power relay controller (SDMCD-16S) from Campbell Scientific. The datalogger is capable of executing a computer code to control the power relay controller for sending electrical power to heat pulse probes, collect temperature measurements from thermocouples via the thermocouple multiplexer, and store measurement and calculated data. The data can be uploaded via a serial (RS-232) communication to a computer for long-term storage and further analysis.

2. Computer codes: A computer code for automating an experiment can be written in CRBasic Editor on a computer. It is then downloaded via a serial (RS-232) communication to the datalogger for running an experiment. A few codes were written for running different experiments.

For more details of the experimental setup and data acquisition system, the reader can refer to Hedayati-Dezfooli's thesis [27]. Brief descriptions of Hedayati-Dezfooli's thesis are shown in Appendix C.

\section{2 - Improvements to the Experimental Apparatus}

In order to obtain more reliable experimental results, a few improvements to the experimental apparatus were made, as follows:

- In many tests, the soil cell is subjected to high temperatures (above $40^{\circ} \mathrm{C}$ ). In order to reduce heat losses to the ambient air from the soil cell during an experiment, insulation is used to cover the entire soil cell assembly, as shown in Fig. 3.2. Because temperatures as high as $90^{\circ} \mathrm{C}$ are involved while the room is at around $23^{\circ} \mathrm{C}$, the entire soil cell assembly is covered by a thick layer of insulation and then in turn covered in by a big wooden box.

- In addition, due to high heat dissipation from the water baths during operations, paper boxes are used as a wall to block off the thermal interference from the water baths to the DAQ system and the soil cell assembly. 
- Due to heavy usage of the water baths, the dials to divert water from internal flow to external water hoses were broken. In order to easily control the water flows between the water baths and the soil cell assembly, a water circuit, which is composed of water hoses, hose insulation, shut-off and diverting valves, were made for the purpose.

- The code for the CRBasic Editor (which controls the DAQ system and tells the DAQ system transfer the experimental data to the computer) was modified so that periodic heat pulses could be made more automatic for longer experimental times.

- More accurate methods to calculate the experimental soil's porosity and moisture content are developed.

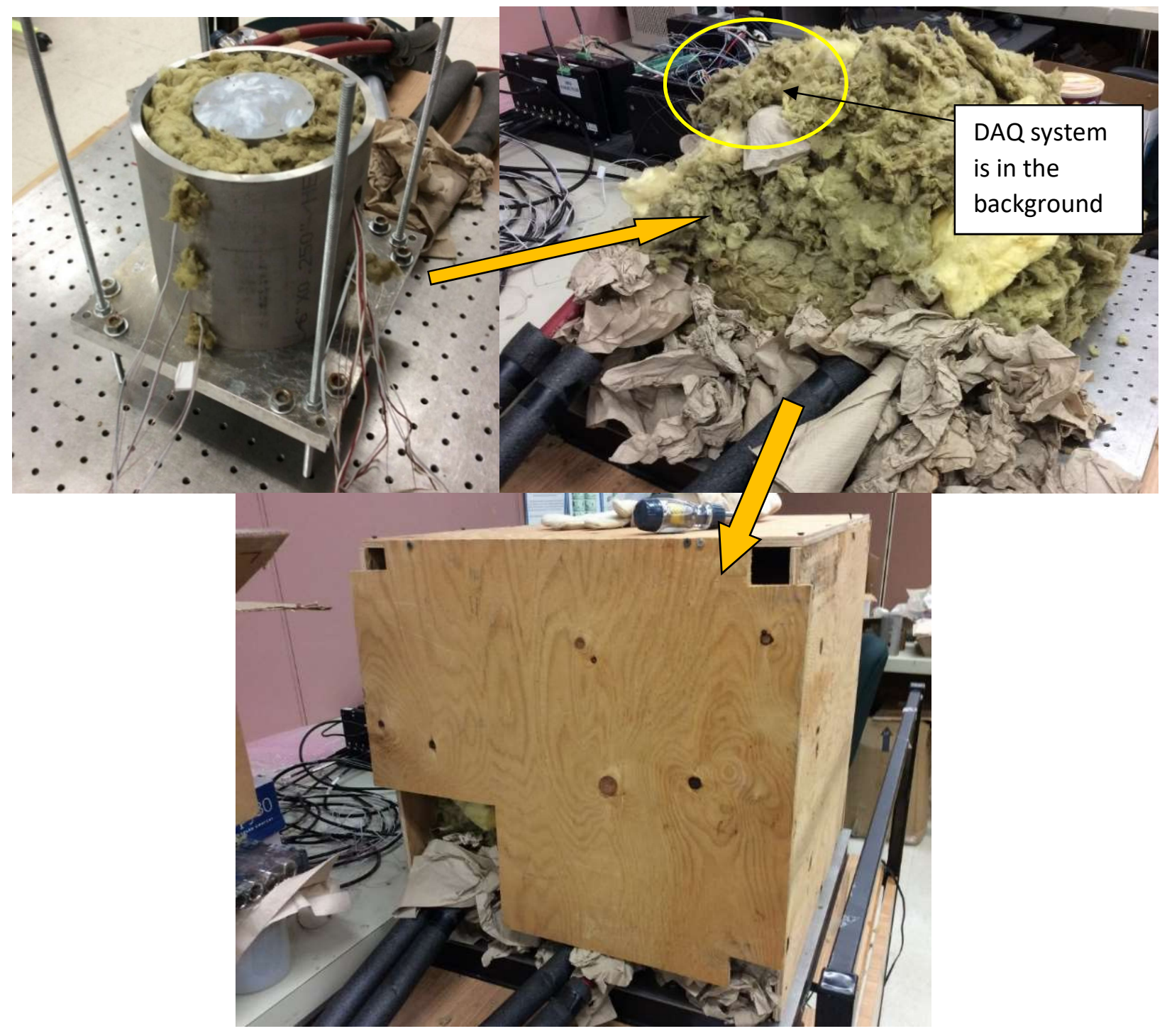

Figure 3.2. Soil cell with insulation. 


\section{3 - Experimental Techniques}

The heat pulse method from Knight et al. [54] is used to obtain the thermal conductivity $(k)$ and volumetric heat capacity $(C)$ of the soil in this thesis. In a heat pulse probe, there are at least two needles (the smaller the better, $\sim 1 \mathrm{~mm}$ in diameter in this thesis). A resistance heating wire is inserted in one needle and a thermocouple is inserted in the other needle. The two needles are 6 $m m$ apart (center to center). An electrical current is passed through the wire for a short time (usually 6 to $8 s$ ) and heats up the wire and the heating needle. The generated heat from the heating needle travels through the soil and is sensed by the needle with the thermocouple. The temperature response of the needle with the thermocouple depends on the thermal properties of the soil and the construction of the probe. With the temperature response data and the construction of the probe, the thermal conductivity and heat capacity of the soil can be calculated.

The experiments can be put into two groups: high thermal gradient testing (or differential heating) and isothermal testing.

\subsection{1 - High Thermal Gradient Testing}

Soil with desired saturation is prepared at least one day before being put into the soil column. Dry soil is compacted layer by layer in a beaker by pressing the top soil surface of the layer and tapping the beaker's wall. The mass of the water to be mixed with the dry soil in the beaker to have the desired saturation is calculated as (see Appendix D for more details):

$$
m_{w}=\rho_{w} \cdot S R \cdot\left(1-\frac{m_{d r y_{-} \text {soil }}}{\forall_{\text {soil }} \cdot \rho_{s}}\right) \cdot \forall_{\text {soil }}
$$

where $S R$ is the nominal saturation ratio, $\forall_{\text {soil }}=600 \mathrm{~mL}$ (i.e., the total volume of the soil in the beaker), $m_{d r y_{-} s o i l}$ is the mass of the dry soil in the beaker before water is added, $\rho_{w}$ is the water density, and $\rho_{s}$ is the solid particle density of the soil. The soils used in the experiments are among 40 Canadian soils obtained from across Canada. Physical characteristics of the soils, including mineral compositions and $\rho_{s}$, can be obtained from Table 4.2. 
The soil and water are poured into a re-sealable plastic bag and manually well mixed. Due to the high water sorption of the dry soil particles, big chunks of wet soil are formed while there are still dry soil particles in the bag when water is first poured into the bag. Consequently, the chunks are manually crushed so that water can reach the dry particles. The crushing and mixing are repeated until no chunk of wet soil is felt. Then, the plastic bag containing the soil mixture is put into another re-sealable plastic bag to prevent water vapour from escaping the bags. After that, the soil mixture and bags are left in room temperature for at least 24 hours so that the water can be more uniformly distributed in the soil mixture.

The prepared soil is weighed for its mixture mass and then compacted in the soil column layer by layer. To reduce moisture evaporation from the wet soil in the soil bag, humidifiers are turned on and located near the work place. First, a stainless-steel lid with an attached heat flux meter is affixed using screws to the bottom of the soil column (i.e., the inner stainless-steel tube). Then, the probe (more details in Fig. 3.3 and Appendix C) at the lowest position of the soil column is inserted first when the first layer of the prepared soil is poured into the soil column. A long flat rod is used to compact the loose soil after the pouring with a slight force. The compacting process is done rather quickly to reduce moisture escape to the room. Subsequently, the probes at higher positions are later inserted according to the filled level of the prepared soil in the soil column. When all five probes are inserted into the soil column and the soil mixture is compacted and leveled on the top by a straight-edge ruler, a stainless-steel lid with an attached heat flux meter is affixed using screws to the top of the soil column which is then sealed. The leftover prepared soil is weighed so that the porosity of the soil mixture in the soil column can be calculated with the following equation (see Appendix D for more details):

$$
\eta_{\text {overall }}=1-\frac{1}{\rho_{s}} \cdot \frac{m_{\text {wet }}}{\left(1+m_{w} / m_{d r y \__{\text {soil }}}\right) \cdot \forall_{\text {column }}}
$$

where $\rho_{s}$ is the density of solid soil particles as tabulated in Table 4.2, $m_{\text {wet }}$ is the mass of the soil mixture in the soil column, $m_{d r y_{-} s o i l}$ is the mass of the dry soil in the beaker before water is added, $m_{w}$ (which can be calculated from Eq. 3.1) is the mass of the added water to the dry soil in the beaker, and $\forall_{\text {column }}=470 \mathrm{~mL}$ is the internal volume of the soil column, excluding the space occupied by the needles of all five probes. 
The experimental moisture (or water) content in the soil can be calculated as (see Appendix D for more details):

$$
\theta_{l_{-} \exp }=\frac{\forall_{w}}{\forall_{\text {column }}}=\frac{m_{\text {wet }}}{\rho_{w} \cdot \forall_{\text {column }}}\left(1-\frac{1}{1+\frac{m_{w}}{m_{\text {dry_soil }}}}\right)
$$

where $m_{w e t}$ is the mass of the soil in the soil mixture in the soil column, $m_{d r y_{-} s o i l}$ is the dry mass of the soil in the beaker, $m_{w}$ is the water mass added to the dry soil in the beaker, $\forall_{\text {column }}=470 \mathrm{~mL}$ is the internal volume of the soil column excluding the space occupied by the needles of all five probes, and $\rho_{w}$ is the water density.

After the soil column is prepared, it is carefully surrounded by an outer stainless-steel tube for isolating the soil column from the ambient and put into the soil cell, both sandwiched between the hot and cold aluminum plates, as shown in Fig. 3.1. Because the soil cell has hot and cold aluminum plates during experimentation, heat can escape from the hot plate and moisture condensation can form at the cold plate, which can significantly affect the measurements. As a result, the entire soil cell assembly is covered with insulation, as shown in Fig. 3.2.

With the five inserted probes and two heat flux meters (HFMs), 12 temperature points can be measured along the soil column from top to bottom. The heat fluxes at the top and bottom of the soil column are measured using the HFMs. However, the thermal properties of the soil and the moisture contents in the soil column are not directly measured as the temperatures are. A computer program based on the heat pulse method from Knight et al. [54] is used to calculate the thermal properties of the soil at each probe, based on the temperature responses from the two side needles of the probe, which are called the temperature-sensing needles, as shown in Fig. 3.3. In general, a heat pulse is generated at the middle needle of the probe, which is called the heat-pulse needle, for about 6 seconds and the temperature responses at the two side needles are recorded every second for about 180 seconds. After that, the two recorded temperature responses are read into the computer program to calculate the thermal properties of the soil. Once the volumetric heat capacity of the soil is calculated, it is then used to calculate the volumetric moisture content of the soil. This will be discussed in Section 4.1 (Eq. 4.4). 


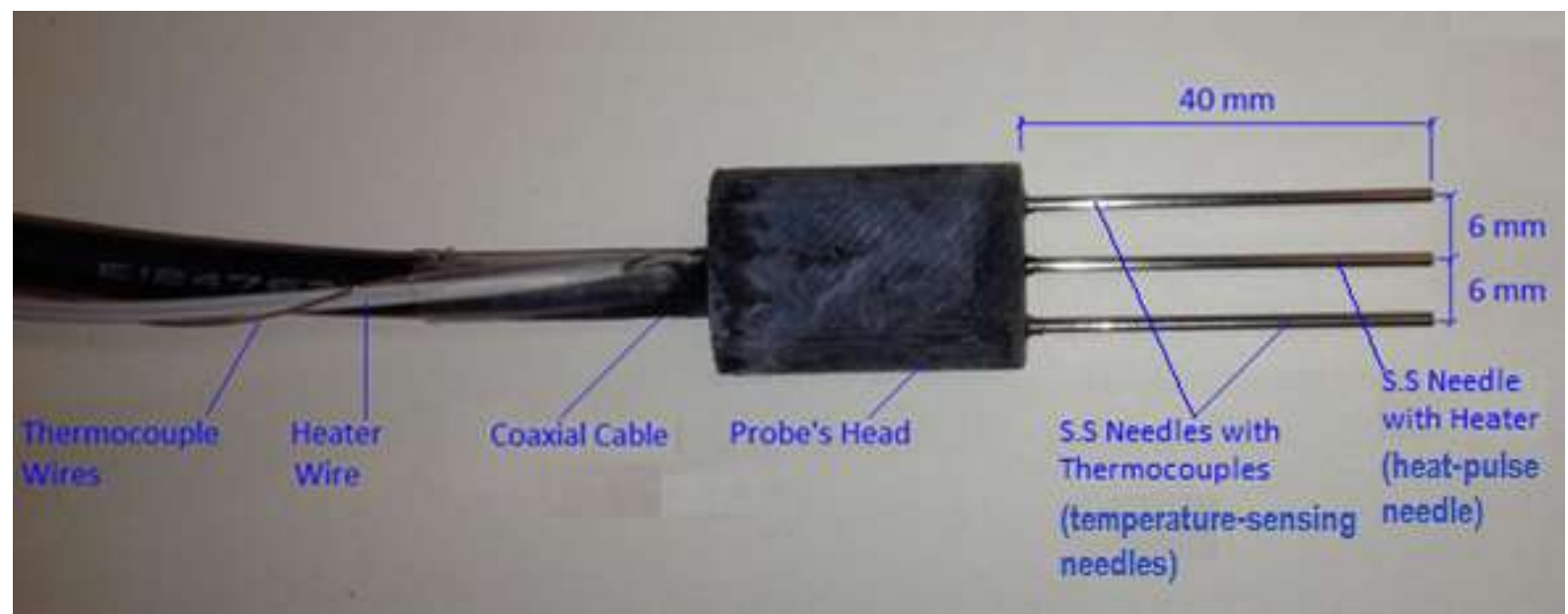

Figure 3.3. An in-house made heat pulse probe.

For the high thermal gradient tests, the hot and cold water baths are usually set at $90^{\circ} \mathrm{C}$ and $10^{\circ} \mathrm{C}$, respectively. This setting results in about $430^{\circ} \mathrm{C} / \mathrm{m}$ of overall temperature gradient over the height of the soil column. Two water baths are used to provide the differential heating. The water in a water bath is first heated to $90^{\circ} \mathrm{C}$ while the water in the other bath is cooled to $10^{\circ} \mathrm{C}$. Then the mechanical switch is manually turned on so that the water from the baths can start to flow through the heating and cooling plates of the soil cell.

\subsection{2 - Isothermal Testing}

These tests are performed when the thermal gradient in the soil column is relatively small and is primarily for exploring the moisture movement in the wet soils due to gravity or the moisture gradients. There are two subcases considered:

1. Soil (with $\mathrm{SR} \approx 0.5$ or field capacity) is prepared and compacted in the soil column using the procedures in Section 3.3.1. With the soil column being positioned horizontally and both water baths being set the same temperature, the temperature of the soil cell is gradually increased from the room temperature by an increment of maximum $2^{\circ} \mathrm{C}$ in every hour until reaching a desired temperature $\left(e . g ., 42^{\circ} \mathrm{C}, 52^{\circ} \mathrm{C}, 62^{\circ} \mathrm{C}\right.$ and so on) so that the thermal gradient is small and will not cause moisture movement in the soil. After reaching a desired high temperature, the soil cell is rotated and positioned vertically. Insulation is still applied as shown in Fig. 3.2. This test is to study the moisture movement in the soil due to the gravity. 
2. The soil column is composed of five different saturation ratios $(0.00,0.25,0.40,0.50$, and $0.70)$ and is prepared and experimented under room temperature. The soil column is split into five volumes, each of which contains a different saturation ratio. For each saturation ratio, approximately $94 \mathrm{~mL}$ of dry soil (whose mass is proportionally calculated from the tested soil in the case of dry heating) is mixed with an appropriate amount of water as determined using Eq. 3.1. The dry soil is first poured into a beaker without any compaction and then into a re-sealable plastic bag. The desired water amount is then weighed and the water is poured into the plastic bag with the dry soil. The bag is then sealed and manually well mixed. With $\mathrm{SR} \approx 0.70$, the bag is put in a microwave oven and heated for about 10 $s$ and manually well mixed again in order to have more uniform water distribution in the soil. Other wet soil bags are not put in the microwave. All bags are left as flat as possible in the room for at least 24 hours so that the moisture can become more uniform throughout the soil in the plastic bag. Later, the soils are put into the soil column with the wettest at the bottom and driest on top. After each wet soil is put into the soil column, a probe is inserted. The soil column is then sealed right after the dry soil has been poured into the top portion of the soil column, compacted and leveled with a straight-edge ruler. After the sealing, the soil column is laid horizontally or vertically upside down (i.e., the wettest part on the top). The upside down case is to test the moisture movements in the soils due to gravity and moisture gradient. The horizontal case is to test the moisture movements due to moisture gradients. The experiments for this subcase can only be done under room temperature because of limitations in sample preparation and testing environment at high temperatures $\left(>40^{\circ} \mathrm{C}\right)$. Medium layer of insulation is applied to reduce the effects fluctuations in the lab room's temperature during day and night times.

\section{4-Summary}

In order to validate the mathematical models of coupled heat and moisture transfer, experimental data are needed. The procedures and apparatus to get the experimental data are described. Basically, the apparatus consists of hardware (a datalogger, a thermocouple multiplexer, a power relay controller, a soil cell, and a computer) and software (CRBasic codes and Excel data files) to record experimental data for further analysis. Experimental procedures for testing are also 
described. The existing experimental apparatus was improved in order to obtain more reliable results. The testing can be split into high thermal gradient and isothermal categories. For the isothermal cases, the thermal gradient is relatively small to cause little to no moisture movement. The isothermal cases are for testing moisture movements due to gravity and moisture gradient. They can be done under room temperature or by gradual heating (increased by $\sim 2{ }^{\circ} \mathrm{C}$ every hour) up to a desired high temperature $\left(>40^{\circ} \mathrm{C}\right)$. Medium to high level of insulation is used to cover the soil cell and prevent heat losses. For the high thermal gradient cases, the experiments are mostly done with insulation to prevent heat losses and possible vapor condensation at the cold end of the soil cell. The soil samples are prepared in plastic bags, left under room temperature for at least 24 hours, and compacted into the soil column layer by layer. Fig. 3.4 summarizes the experimental methodology in this thesis.

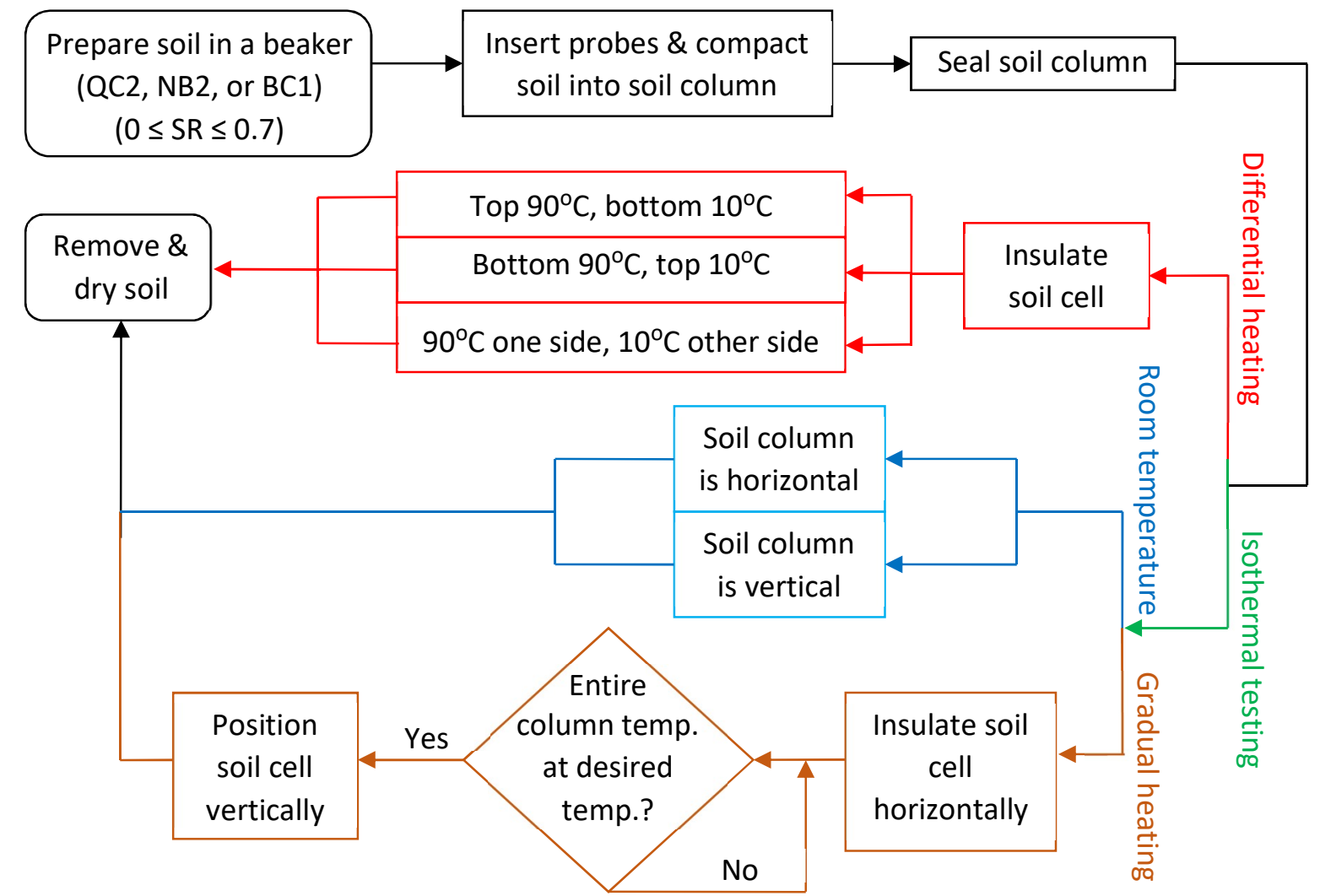

Figure 3.4. Summary of experimental methodology in this thesis.

In the next chapter, experimental results will be shown and discussed. Uncertainties of the experiments are also reported. 


\section{CHAPTER 4 - EXPERIMENTAL RESULTS AND DISCUSSIONS}

\section{1 - Uncertainty Analysis}

For every experiment, there are always uncertainties from many sources such as reading uncertainties, power fluctuations, equipment's manufacturing tolerances, and the propagation of uncertainties due to data reduction. Regardless of how carefully the experiments are setup and done, uncertainties still exist. As a result, all experimental data contain uncertainties which can grouped as systematic and random uncertainties. According to ISO GUM guidelines [55], the systematic uncertainty is a result of the propagation of uncertainties due to the equations used to calculate the desired values using the measured data from the apparatus. Meanwhile, the random uncertainty is from the fluctuations and tolerances of the components of the apparatus.

\subsection{1 - Systematic Uncertainty}

In the present study, the thermal properties of soils are actually evaluated by a more accurate computer program based on the heat pulse method from Knight et al. [54], which takes into account the thermal capacitance of the needles of a heat pulse probe. Therefore, the following uncertainty analysis for the thermal properties of soils may be a bit conservative due to the less accurate formulations by Kluitenberg et al. [56], which do not take into account the thermal capacitance of the needles of a heat pulse probe.

The thermal diffusivity of the soil using the heat pulse method can be calculated as [56]:

$$
D_{T}=\frac{d_{\text {center }}^{2}}{4}\left[\frac{\frac{1}{t_{m}-t_{0}}-\frac{1}{t_{m}}}{\ln \left(\frac{t_{m}}{t_{m}-t_{0}}\right)}\right]
$$

where $t_{\mathrm{m}}$ is the time at which the temperature rise $(\Delta T)$ of the temperature-sensing needle reaches maximum, as shown in Fig. 4.1, $d_{\text {center }}$ is the centre-to-centre distance between the heat-pulse needle and a temperature-sensing needle, and $t_{0}$ is the heating duration of the heat pulse needle. 


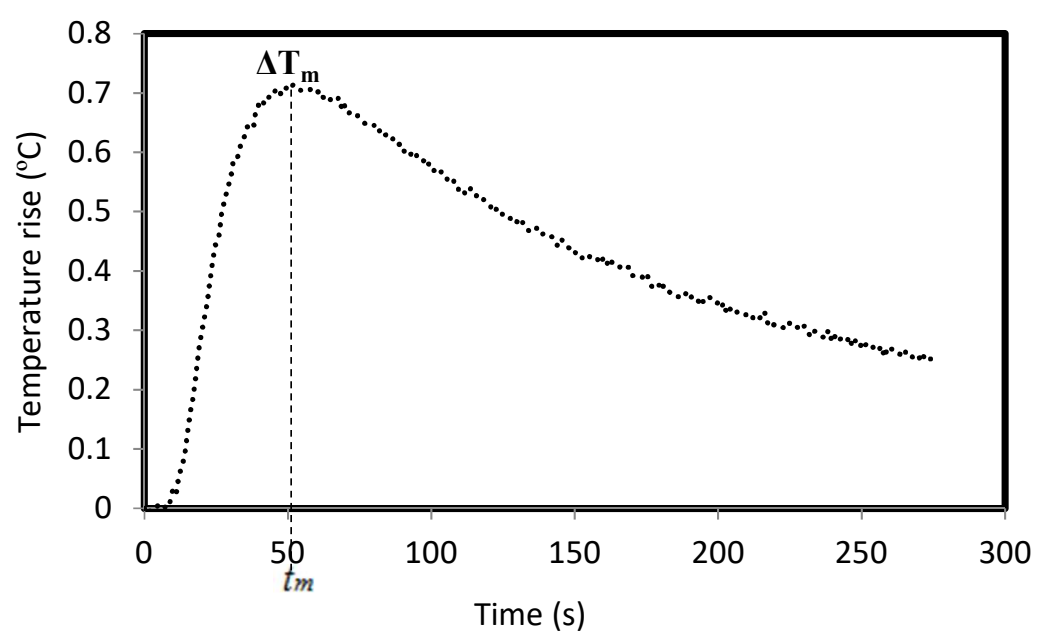

Figure 4.1. A sample of temperature response of a temperature-sensing needle.

The volumetric heat capacity of the soil can be approximately expressed as [56]:

$$
C=\frac{\dot{q}_{w}}{4 \pi D_{T} \Delta T_{m}}\left[E i\left(\frac{-d_{c e n t e r}^{2}}{4 D_{T}\left(t_{m}-t_{0}\right)}\right)-E i\left(\frac{-d_{\text {center }}^{2}}{4 D_{T} t_{m}}\right)\right]
$$

where $\Delta T_{m}$ is the maximum temperature rise of the temperature-sensing needle at time $t_{m}$, and $\dot{q}_{w}$ is the heating power per unit length of the heat pulse needle.

The thermal conductivity of the soil is then calculated as:

$$
k=C \cdot D_{T}
$$

The soil moisture content can be approximately determined as (see Appendix D for more details):

$$
\theta_{l} \approx \frac{C-C_{d r y}}{\rho_{w} c_{w}}
$$

Due to the difficulties in sample preparations and soil compactions, the moist and dry porosities of the same soil type cannot be made the same. As a result, for each soil type, three dry porosities are experimentally prepared to obtain the corresponding thermal conductivities and 
volumetric heat capacities. The dry soil with the lowest porosity value gets the tightest compaction while the soil with highest porosity value gets no compaction. The porosity value of the moist soil is used to approximately interpolate its dry volumetric heat capacity.

The propagation uncertainties due to data reduction for determining $D_{T}, C, k$, and $\theta_{l}$ from Eqs. 4.1 to 4.4 can be theoretically assessed using the root-sum-square (RSS) method, respectively, as follows [27]:

$$
\begin{gathered}
\frac{\delta D_{T}}{D_{T}} \approx\left[\left(2 \frac{\delta d_{\text {center }}}{d_{\text {probe }}}\right)^{2}+\left(\tau_{1} \frac{\delta t_{m}}{t_{m}}\right)^{2}+\left(\left(1+\tau_{1}\right) \frac{\delta t_{0}}{t_{0}}\right)^{2}\right]^{0.5} \\
\left.\frac{\delta C}{C} \approx\left[\left(\frac{\delta \dot{q}_{w}}{\dot{q}_{w}}\right)^{2}+\left(\frac{\delta \Delta T_{m}}{\Delta T_{m}}\right)^{2}+\left(2 \frac{\delta d_{\text {center }}}{d_{\text {probe }}}\right)^{2}+\left(\tau_{2} \frac{\delta t_{m}}{t_{m}}\right)^{2}+\left(1-\tau_{2}\right) \frac{\delta t_{0}}{t_{0}}\right)^{2}\right]^{0.5} \\
\left.\frac{\delta k}{k}=\left[\left(\frac{\delta C}{C}\right)^{2}+\left(\frac{\delta D_{T}}{D_{T}}\right)^{2}\right]^{0.5}\right]^{2} \\
\frac{\delta \theta_{l}}{\theta_{l}}=\left[\left(\frac{\delta C}{C-C_{d r y}}\right)^{2}+\left(\frac{\delta C_{d r y}}{C-C_{d r y}}\right)^{2}+\left(\frac{\delta \rho_{w}}{\rho_{w}}\right)^{2}+\left(\frac{\delta c_{w}}{c_{w}}\right)^{2}\right]^{0.5}
\end{gathered}
$$

where $\tau_{1}=\frac{4 D_{T} \cdot t_{m}}{d_{\text {center }}^{2}}-\frac{t_{m}}{t_{m}-t_{0}}-1, \tau_{2}=-\tau_{1}\left(1+\tau_{3}\right), \tau_{3}=\frac{\exp \left(\tau_{5}\right)-\exp \left(\tau_{6}\right)}{\operatorname{Ei}\left(\tau_{5}\right)-\operatorname{Ei}\left(\tau_{6}\right)}, \tau_{5}=\frac{-d_{\text {center }}^{2}}{4 D_{T}\left(t_{m}-t_{0}\right)}$, and $\tau_{6}=\frac{-d_{c e n t e r}^{2}}{4 D_{T} t_{m}}$

Due to manufacturing tolerances and variations, all components in the apparatus cannot be made to their exact dimensions. The tolerances and uncertainties of some components in the apparatus are listed in Table 4.1. Three soils from the 40 known Canadian soils [57] are selected in this research study, namely: QC2, NB2 and BC1. They represent three different textural classes of coarse, medium and fine soils, respectively. Their detailed information is listed in Table 4.2. The table also shows the soils' geographical locations that they came from, grain size distributions 
(GSDs), solid densities, textures, and classes. The solid density of a soil $\rho_{s}$ is the density of the solid particles of the soils without the pores. The summary of experimental values and theoretical uncertainties for $D_{T}, C, k$, and $\theta_{l}$ of the three soils are shown in Tables 4.3, 4.4, and 4.5.

Table 4.1. Tolerances and uncertainties of the components in the apparatus

\begin{tabular}{|c|c|c|c|c|c|c|c|c|c|c|}
\hline $\begin{array}{c}\delta T_{m} \\
\left({ }^{\circ} \mathrm{C}\right)\end{array}$ & $\begin{array}{c}\delta d_{\text {center }} \\
(\mathrm{mm})\end{array}$ & $\begin{array}{c}d_{\text {center }} \\
(\mathrm{mm})\end{array}$ & $\begin{array}{c}\delta t_{m} \\
(\mathrm{~s})\end{array}$ & $\begin{array}{c}\delta t_{0} \\
(\mathrm{~s})\end{array}$ & $\begin{array}{c}t_{0} \\
(s)\end{array}$ & $\begin{array}{c}\delta \dot{q}_{w} \\
(\mathrm{~J} / \mathrm{m} \cdot \mathrm{s})\end{array}$ & $\frac{\delta m_{w}}{m_{w}}$ & $\frac{\delta \rho_{w}}{\rho_{w}}$ & $\frac{\delta c_{w}}{c_{w}}$ & $\frac{\delta \forall_{\text {soil }}}{\forall_{\text {soil }}}$ \\
\hline \pm 0.50 & \pm 0.10 & 6.0 & \pm 0.75 & \pm 0.01 & 6.00 & \pm 0.16 & $\pm 0.1 \%$ & $\pm 0.1 \%$ & $\pm 0.1 \%$ & $\pm 1.0 \%$ \\
\hline
\end{tabular}

Table 4.2. Basic physical characteristics of three Canadian soils used in this study [57]

\begin{tabular}{|c|c|c|c|c|c|c|c|c|}
\hline \multirow{2}{*}{$\begin{array}{c}\text { Soil } \\
\text { ID }\end{array}$} & \multirow{2}{*}{ Name } & \multirow{2}{*}{ Location } & \multirow{2}{*}{ Texture } & \multirow{2}{*}{ Class } & \multirow{2}{*}{$\begin{array}{c}\rho_{s} \\
\left(\frac{k g}{m^{3}}\right)\end{array}$} & \multicolumn{3}{|c|}{ GSD (Mass \%) } \\
\hline & & & & & & Clay & Silt & Sand \\
\hline QC2 & $\begin{array}{l}\text { Macdonald campus } \\
\text { "Field 9" }\end{array}$ & $\begin{array}{l}\text { McGill U. } \\
\text { Research site }\end{array}$ & $\begin{array}{l}\text { Loamy } \\
\text { sand }\end{array}$ & $\begin{array}{c}\text { Coarse } \\
\text { soil }\end{array}$ & 2693 & 3.30 & 17.4 & 79.3 \\
\hline NB2 & Victoria & $\begin{array}{c}\text { Northern } \\
\text { Victoria County }\end{array}$ & $\begin{array}{l}\text { Silt } \\
\text { loam }\end{array}$ & $\begin{array}{l}\text { Medium } \\
\text { soil }\end{array}$ & 2540 & 16.6 & 83.4 & 0.00 \\
\hline BC1 & FSJ \#1 & Fort St. James & $\begin{array}{l}\text { Silty } \\
\text { clay }\end{array}$ & $\begin{array}{r}\text { Fine } \\
\text { soil }\end{array}$ & 2740 & 41.8 & 58.2 & 0.00 \\
\hline
\end{tabular}

From Tables 4.3, 4.4, and 4.5, it can be seen that as the moisture content increases, the thermal diffusivity of the soil is harder to be obtained accurately than the volumetric heat capacity is. On the other hand, the moisture content can be more accurately obtained with increasing moisture content. The reason is that the difference in the volumetric heat capacities between the wet and dry soils, i.e. $C-C_{d r y}$ in Eq. $4.5 \mathrm{~d}$, is small when the wet soil has low moisture content, therefore, resulting in high theoretical uncertainty for calculating low volumetric moisture content. In addition, the experimental temperature can play a role in the accuracy of the obtained data. The higher uncertainties happen at higher temperatures because at higher temperatures the moisture has more energy to flow through the pore space of the soil, making the measurements less accurate. Lower moisture content has more air space for the moisture to move, making it harder to correctly measure the moisture. As a result, higher measurement uncertainties of the moisture content happen in drier soils. 
Table 4.3. Experimental values and systematic uncertainties for $D_{T}, C, k$, and $\theta_{l}$ at $\approx 80^{\circ} C$

\begin{tabular}{|c|c|c|c|c|c|c|c|c|c|c|}
\hline $\begin{array}{c}\text { Soil } \\
\text { ID }\end{array}$ & $S R$ & $\eta$ & $\begin{array}{c}D_{T} \\
\left(\mathrm{~mm}^{2} / \mathrm{s}\right)\end{array}$ & $\begin{array}{c}C \\
\left(M J / m^{3} \cdot \mathrm{K}\right)\end{array}$ & $\begin{array}{c}k \\
(\mathrm{~W} / m \cdot \mathrm{K})\end{array}$ & $\begin{array}{c}\theta_{l} \\
\left(m^{3} / m^{3}\right) \\
\end{array}$ & $\frac{\delta D_{T}}{D_{T}}$ & $\frac{\delta C}{C}$ & $\frac{\delta k}{k}$ & $\frac{\delta \theta_{l}}{\theta_{l}}$ \\
\hline \multirow{3}{*}{ Чิ } & 0.00 & 0.45 & 0.19 & 1.62 & 0.31 & 0.000 & $3.40 \%$ & $3.43 \%$ & $4.83 \%$ & - \\
\hline & 0.25 & 0.49 & 0.99 & 1.81 & 1.79 & 0.121 & $8.89 \%$ & $3.45 \%$ & $9.54 \%$ & $44.0 \%$ \\
\hline & 0.50 & 0.44 & 1.38 & 2.06 & 2.84 & 0.218 & $10.9 \%$ & $3.71 \%$ & $11.5 \%$ & $21.5 \%$ \\
\hline \multirow{3}{*}{$\stackrel{\approx}{z}$} & 0.00 & 0.57 & 0.15 & 1.59 & 0.24 & 0.000 & $3.47 \%$ & $3.43 \%$ & $4.88 \%$ & - \\
\hline & 0.25 & 0.56 & 0.69 & 1.85 & 1.28 & 0.139 & $5.72 \%$ & $3.46 \%$ & $6.69 \%$ & $32.6 \%$ \\
\hline & 0.50 & 0.55 & 0.91 & 2.28 & 2.26 & 0.272 & $6.07 \%$ & $3.61 \%$ & $7.06 \%$ & $14.4 \%$ \\
\hline \multirow{3}{*}{$\bar{U}_{0}$} & 0.00 & 0.59 & 0.16 & 1.72 & 0.28 & 0.000 & $3.63 \%$ & $3.42 \%$ & $4.99 \%$ & - \\
\hline & 0.25 & 0.56 & 0.37 & 2.38 & 0.88 & 0.139 & $4.33 \%$ & $3.43 \%$ & $5.53 \%$ & $15.0 \%$ \\
\hline & 0.50 & 0.51 & 0.59 & 2.70 & 1.60 & 0.252 & $9.03 \%$ & $4.19 \%$ & $9.96 \%$ & $12.9 \%$ \\
\hline
\end{tabular}

Table 4.4. Experimental values and systematic uncertainties for $D_{T}, C, k$, and $\theta_{l}$ at $\approx 50^{\circ} C$

\begin{tabular}{|c|c|c|c|c|c|c|c|c|c|c|}
\hline $\begin{array}{l}\text { Soil } \\
\text { ID }\end{array}$ & $S R$ & $\eta$ & $\begin{array}{c}D_{T} \\
\left(\mathrm{~mm}^{2} / \mathrm{s}\right)\end{array}$ & $\begin{array}{c}C \\
\left(M J / m^{3} \cdot \mathrm{K}\right)\end{array}$ & $\begin{array}{c}k \\
(\mathrm{~W} / m \cdot \mathrm{K})\end{array}$ & $\begin{array}{c}\theta_{l} \\
\left(\mathrm{~m}^{3} / \mathrm{m}^{3}\right)\end{array}$ & $\frac{\delta D_{T}}{D_{T}}$ & $\frac{\delta C}{C}$ & $\frac{\delta k}{k}$ & $\frac{\delta \theta_{l}}{\theta_{l}}$ \\
\hline \multirow{3}{*}{ Uี } & 0.00 & 0.45 & 0.17 & 1.62 & 0.28 & 0.000 & $3.44 \%$ & $3.53 \%$ & $4.93 \%$ & - \\
\hline & 0.25 & 0.49 & 0.76 & 1.86 & 1.42 & 0.121 & $3.76 \%$ & $3.57 \%$ & $5.19 \%$ & $36.1 \%$ \\
\hline & 0.50 & 0.44 & 0.95 & 2.20 & 2.08 & 0.218 & $3.81 \%$ & $3.60 \%$ & $5.24 \%$ & $16.7 \%$ \\
\hline \multirow{3}{*}{$\tilde{z}$} & 0.00 & 0.57 & 0.15 & 1.30 & 0.22 & 0.000 & $3.44 \%$ & $3.43 \%$ & $4.86 \%$ & - \\
\hline & 0.25 & 0.56 & 0.58 & 1.67 & 0.97 & 0.139 & $4.37 \%$ & $3.57 \%$ & $5.65 \%$ & $22.0 \%$ \\
\hline & 0.50 & 0.55 & 0.62 & 2.08 & 1.29 & 0.272 & $4.76 \%$ & $3.58 \%$ & $5.96 \%$ & $11.9 \%$ \\
\hline \multirow{3}{*}{$\bar{\Xi}$} & 0.00 & 0.59 & 0.14 & 1.66 & 0.24 & 0.000 & $3.71 \%$ & $3.53 \%$ & $5.12 \%$ & - \\
\hline & 0.25 & 0.56 & 0.33 & 2.25 & 0.75 & 0.139 & $4.53 \%$ & $3.53 \%$ & $5.74 \%$ & $16.4 \%$ \\
\hline & 0.50 & 0.51 & 0.51 & 2.38 & 1.21 & 0.252 & $5.10 \%$ & $3.55 \%$ & $6.21 \%$ & $14.5 \%$ \\
\hline
\end{tabular}


Table 4.5. Experimental values and systematic uncertainties for $D_{T}, C, k$, and $\theta_{l}$ at $\approx 20^{\circ} \mathrm{C}$

\begin{tabular}{|c|c|c|c|c|c|c|c|c|c|c|}
\hline Soil & $S R$ & $\eta$ & $\begin{array}{c}D_{T} \\
\left(\mathrm{~mm}^{2} / \mathrm{s}\right)\end{array}$ & $\begin{array}{c}C \\
\left(M J / m^{3} \cdot \mathrm{K}\right)\end{array}$ & $\begin{array}{c}k \\
(\mathrm{~W} / m \cdot \mathrm{K})\end{array}$ & $\begin{array}{c}\theta_{l} \\
\left(m^{3} / m^{3}\right)\end{array}$ & $\frac{\delta D_{T}}{D_{T}}$ & $\frac{\delta C}{C}$ & $\frac{\delta k}{k}$ & $\frac{\delta \theta_{l}}{\theta_{l}}$ \\
\hline \multirow{3}{*}{ ত̂ } & 0.00 & 0.45 & 0.19 & 1.42 & 0.27 & 0.000 & $3.42 \%$ & $4.19 \%$ & $5.41 \%$ & - \\
\hline & 0.25 & 0.49 & 0.58 & 1.82 & 1.06 & 0.121 & $3.36 \%$ & $3.93 \%$ & $5.17 \%$ & $22.6 \%$ \\
\hline & 0.50 & 0.44 & 0.59 & 2.80 & 1.66 & 0.218 & $3.46 \%$ & $3.83 \%$ & $5.16 \%$ & $8.75 \%$ \\
\hline \multirow{3}{*}{$\tilde{z}$} & 0.00 & 0.57 & 0.17 & 1.07 & 0.18 & 0.000 & $3.47 \%$ & $4.18 \%$ & $5.43 \%$ & - \\
\hline & 0.25 & 0.56 & 0.38 & 1.85 & 0.70 & 0.139 & $3.93 \%$ & $3.79 \%$ & $5.45 \%$ & $11.5 \%$ \\
\hline & 0.50 & 0.55 & 0.44 & 2.44 & 0.84 & 0.272 & $4.33 \%$ & $3.94 \%$ & $5.85 \%$ & $8.11 \%$ \\
\hline \multirow{3}{*}{ 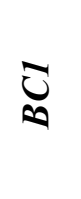 } & 0.00 & 0.59 & 0.13 & 1.58 & 0.21 & 0.000 & $3.51 \%$ & $3.96 \%$ & $5.29 \%$ & - \\
\hline & 0.25 & 0.56 & 0.27 & 2.19 & 0.59 & 0.139 & $4.36 \%$ & $4.00 \%$ & $5.91 \%$ & $17.0 \%$ \\
\hline & 0.50 & 0.51 & 0.41 & 2.15 & 0.89 & 0.252 & $3.63 \%$ & $4.07 \%$ & $5.45 \%$ & $18.2 \%$ \\
\hline
\end{tabular}

\subsection{2 - Random Uncertainty}

When an experiment is run, there are many uncertainties within the apparatus itself. During the manufacturing processes of the apparatus, components with perfect dimensions cannot be made. For example, when filling the stainless-steel needles of the heat pulse probe with liquid epoxy, the exact location of the thermocouple bead of copper and constantan wires inside the needle cannot be precisely known but can only be estimated. The heat pulse generator also has built-in tolerances. Another example is soil sample preparation and compaction in the soil column. Despite consistent and best effort, variation of moisture content around a nominal SR can be expected. As a result, even with same testing conditions, identically repeated tests can produce slight variations in the recorded data.

Because of the slow transport phenomena in soils, many tests are run for at least 18 hours. In addition, wet soils usually cannot be repeatedly tested without preparing new samples because the moisture distributions in the soils change after each trial. Old wet soil in the soil cell has to be removed and new wet soil has to be compacted into the soil cell, which can take from three to eight hours to carefully prepare each experiment. In spite of such laborious and time-consuming work, 
all initial measurements are still repeatedly done 10 times by sending a heat pulse to each probe for 10 times and calculating the thermal properties for 10 times.

The standard deviation of the mean can be expressed as:

$$
S_{\bar{x}}=\sqrt{\sum_{i=1}^{n} \frac{\left(x_{i}-\bar{x}\right)^{2}}{n(n-1)}}
$$

Table 4.6 shows the standard deviations of the means from the 10 needles of five heat pulse probes used in the study. The average experimental uncertainties for $k$ and $C$ are $4.64 \%$ and 3.72\% respectively. Due to the long and exhaustive soil preparations and measurements, repeated measurements under transient conditions cannot be done. In other words, once started, an experiment is not repeated. Similar soil properties and temperature responses under transient conditions are not measured again. In addition, since $\theta_{l}$ is calculated using $C$, the experimental uncertainty for $\theta_{l}$ is assumed to be that of $C$.

Table 4.6. Highest standard deviations of the mean for $k$ and $C$ from 10 needles of 5 heat pulse probes

\begin{tabular}{|c|c|c|c|c|c|c|c|c|c|c|c|}
\hline $\begin{array}{c}\text { Soil } \\
\text { ID } \\
\end{array}$ & Needle & 1 & 2 & 3 & 4 & 5 & 6 & 7 & 8 & 9 & 10 \\
\hline$Q C 2$ & \multirow{3}{*}{$\frac{\partial k}{k}$} & $3.30 \%$ & $4.60 \%$ & $3.24 \%$ & $4.39 \%$ & $3.32 \%$ & $3.65 \%$ & $2.31 \%$ & $5.57 \%$ & $6.56 \%$ & $3.05 \%$ \\
\hline$N B 2$ & & $5.87 \%$ & $6.58 \%$ & $3.25 \%$ & $4.69 \%$ & $3.61 \%$ & $4.01 \%$ & $5.04 \%$ & $4.95 \%$ & $6.48 \%$ & $3.19 \%$ \\
\hline$B C 1$ & & $5.25 \%$ & $6.60 \%$ & $3.88 \%$ & $5.52 \%$ & $6.08 \%$ & $5.18 \%$ & $5.75 \%$ & $3.32 \%$ & $4.08 \%$ & $5.70 \%$ \\
\hline$Q C 2$ & \multirow{3}{*}{$\frac{\delta C}{C}$} & $3.12 \%$ & $4.62 \%$ & $3.43 \%$ & $3.87 \%$ & $2.13 \%$ & $2.24 \%$ & $2.33 \%$ & $4.57 \%$ & $5.53 \%$ & $3.17 \%$ \\
\hline NB2 & & $3.44 \%$ & $5.10 \%$ & $3.95 \%$ & $2.46 \%$ & $2.31 \%$ & $2.46 \%$ & $2.98 \%$ & $3.62 \%$ & $4.29 \%$ & $2.75 \%$ \\
\hline$B C 1$ & & $3.87 \%$ & $5.79 \%$ & $3.76 \%$ & $4.17 \%$ & $4.81 \%$ & $4.58 \%$ & $4.62 \%$ & $3.54 \%$ & $3.63 \%$ & $4.56 \%$ \\
\hline
\end{tabular}

\subsection{3 - Overall uncertainty}

The overall uncertainty for $95 \%$ confidence level can be calculated as:

$$
U_{0.95}=\sqrt{B_{\bar{x}}^{2}+\left(t S_{\bar{x}}\right)^{2}}
$$


where $S_{\bar{x}}$ is the standard deviation of the mean (defined in Eq. 4.6), $t$ is the student's $t$ multiplier for $95 \%$ confidence level (=2.262 for 10 samples), $n$ is the number of repeated measurements, and $B_{\bar{x}}$ is the systematic uncertainty from Section 4.1.1.

Combining the systematic uncertainty with the random one and being on the conservative side, the maximum overall uncertainties at 95\% confidence level are $15.5 \%$ and $9.20 \%$ for $k$ an $C$ respectively. The maximum uncertainty of $\theta_{l}$ is estimated to be $48.6 \%$ at $\mathrm{SR}=0.25$ and reduces to $29.9 \%$ at $\mathrm{SR}=0.5$.

\section{2 - Measured Thermal Properties of Dry QC2, NB2, and BC1 Soils}

When the temperature changes, the thermal properties of the soils also vary. Table 4.7 shows the fitted coefficients of thermal properties of dry soils as linear functions of temperature, as follows:

$$
\Xi_{X}=a_{X} \times 10^{-4} T+b_{X}
$$

where $\Xi_{X}$ represents the thermal conductivity (in $W / m \cdot K$ ) or volumetric heat capacity (in $M J /$ $\left.m^{3} \cdot K\right)$ and $T$ is in ${ }^{\circ} \mathrm{C}$.

Table 4.7. Fitted coefficients of Eq. 4.8 for $k$ and $C$ of dry soils $\left(22^{\circ} \mathrm{C} \leq T \leq 80^{\circ} \mathrm{C}\right)$ and the corresponding $R^{2}$ values.

\begin{tabular}{|c|c|c|c|c|c|c|c|c|c|c|}
\hline \multicolumn{2}{|c|}{ Soil ID } & \multicolumn{3}{|c|}{ QC2 } & \multicolumn{3}{c|}{ NB2 } & \multicolumn{3}{c|}{ BC1 } \\
\hline \multicolumn{2}{|c|}{ Porosity, $\eta$} & $\mathbf{0 . 4 2}$ & $\mathbf{0 . 4 5}$ & $\mathbf{0 . 5 4}$ & $\mathbf{0 . 5 1}$ & $\mathbf{0 . 5 7}$ & $\mathbf{0 . 5 9}$ & $\mathbf{0 . 5 1}$ & $\mathbf{0 . 5 9}$ & $\mathbf{0 . 6 4}$ \\
\hline \multirow{2}{*}{$\left.\begin{array}{c}k \\
\left(\frac{W}{m \cdot K}\right)\end{array}\right)$} & $a_{X}$ & -5.09 & 7.44 & 4.84 & 9.90 & 7.37 & 6.56 & 12.0 & 8.63 & 10.1 \\
\cline { 2 - 11 } & $b_{X}$ & 0.32 & 0.25 & 0.19 & 0.23 & 0.18 & 0.18 & 0.19 & 0.18 & 0.16 \\
\cline { 2 - 11 } & $R^{2}$ & 0.932 & 0.927 & 0.929 & 0.927 & 0.928 & 0.930 & 0.929 & 0.926 & 0.928 \\
\hline \multirow{2}{*}{$\left(\begin{array}{c}M J \\
m^{3} \cdot K\end{array}\right)$} & $a_{X}$ & 78.7 & 70.5 & 60.5 & 65.5 & 73.2 & 59.6 & 44.2 & 57.7 & 41.9 \\
\cline { 2 - 11 } & $b_{X}$ & 1.36 & 1.13 & 1.00 & 1.04 & 0.96 & 0.97 & 1.55 & 1.24 & 1.25 \\
\cline { 2 - 10 } & $R^{2}$ & 0.954 & 0.952 & 0.948 & 0.950 & 0.947 & 0.949 & 0.952 & 0.951 & 0.954 \\
\hline
\end{tabular}


As seen from Table 4.7, most thermal properties of soils increase as the temperature rises. Also, as an example, Fig. 4.2 shows the trends of measured thermal properties of dry QC2 soil as the temperature changes at three different porosity values. The only thermal property that slightly lowers with increasing temperature is the thermal conductivity of QC2 soil with the lowest porosity of 0.42 . The reason is that QC2 has a very high sand content (79.3\% by mass, as shown in Table 4.1) which has high quartz content ( $42 \%$ by solid volume [57]). Quartz has decreasing thermal conductivity as the temperature rises. With higher porosity, there are more pore spaces in QC2 and the insulating effect of air becomes more prominent. Therefore, for QC2 with porosities of 0.45 and 0.54 , air with its increasing thermal conductivity with rising temperature has a dominating effect on the thermal conductivity of the dry QC2.

As it can be seen from Fig. 4.2, the thermal properties display linear increases with the temperature. Other fitted coefficients in other tested soils show similar trends and values. Although the $\mathrm{R}^{2}$ values in Fig. 4.2 appear a little smaller than unity, the behaviors of the data points tend to follow the predicted models well. As indicated by Frost [58], high $\mathrm{R}^{2}$ values may not be good and low $\mathrm{R}^{2}$ values may not be bad. The residual plot (similar to Fig. 4.2) can better clarify whether a fitted model is good or not. A low $\mathrm{R}^{2}$ value is most problematic when predictions with high precisions are needed. 

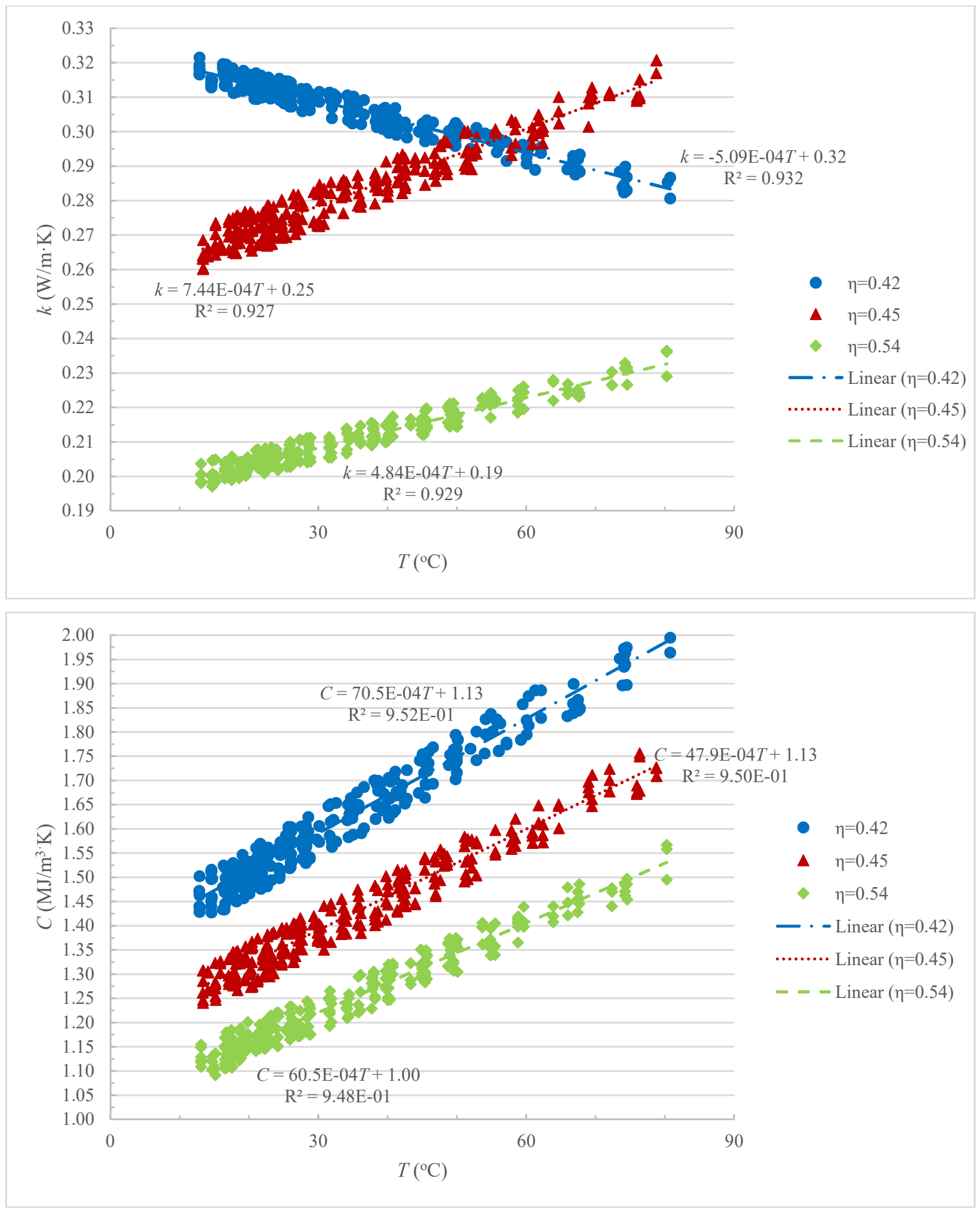

Figure 4.2. Thermal properties of dry QC2 soil ( $\eta=0.42,0.45$ and 0.54) with fitted linear relations of temperature and $R^{2}$ values. 


\section{3 - Measured Thermal Properties of Wet Soils}

$$
\text { 4.3.1-SR } \approx 0.25
$$

The measured thermal properties of the wet soils $(\mathrm{SR} \approx 0.25)$ at room temperature $\left(T \approx 22^{\circ} \mathrm{C}\right)$ are shown in Table 4.8. The last row in the table is from Tarnawski et al. [57]. As they can be seen from Table 4.8 and Fig. 4.3, the more porous the soil is, the lower the thermal conductivity and heat capacity become, following a linear trend. Comparing the measured thermal conductivities in this study with those from Tarnawski et al. [63], the differences are generally not much (less than $20 \%$ ). The first reason for the differences is that the thermal conductivities from Tarnawski et al. are obtained from the single-needle thermal conductivity probe (TCP) which is based on the lineheat-source theory which does not account for the size and construction of the measuring probe. Meanwhile, the heat pulse method of Knight et al. [54], which accounts for the construction of the heat pulse probe, is used to obtain the thermal properties in this thesis. When there are more parameters to be considered, the equation to calculate the experimental uncertainty (e.g., Eqs. 4.5 and 4.7) will produce a bigger number. The second reason is that Tarnawski et al. only discarded the first 20 seconds of probe temperature response data in their analysis for the calculation of the thermal conductivity. According to Dang and Leong [59], data up to 60 seconds or more has to be discarded in order to obtain more accurate thermal conductivity as the exponential integral function in the solution of the line-heat-source theory can be better approximated using the logarithmic function at a longer time. Tarnawski et al. used the approximated exponential integral function to calculate the thermal conductivity. The third reason can be from the actual SRs of the soils in this thesis. During soil preparation processes, it was that the SR of the wet soil in the beaker was different from the wet soil in the soil column due to vaporization during the soil compaction. Consequently, the actual SR of the wet soil in the beaker was increased so that the SR of the wet soil in the soil column was close to the nominal value. Percent differences between the values in this thesis and the corresponding values from Tarnawski et al. could not be made because the porosities of the soils were mostly different. The reader is advised to make appropriate own comparisons between the $k$ values from this thesis and those from Tarnawski et al. in Tables 4.8, 4.9, and 4.12. 
Table 4.8. Measured thermal properties of wet soils ( $S R \approx 0.25$, room temperature).

\begin{tabular}{|c|c|c|c|c|c|c|c|c|}
\hline \multicolumn{2}{|c|}{$Q \boldsymbol{C} 2(\boldsymbol{S R} \approx \mathbf{0 . 2 5})$} & \multicolumn{3}{c|}{ NB2 $(\mathbf{S R} \approx \mathbf{0 . 2 5})$} & \multicolumn{3}{c|}{ BC1 (SR $\approx 0.25)$} \\
\hline$\eta$ & $\left(\frac{W}{m \cdot K}\right)$ & $\left(\frac{M J}{m^{3} \cdot K}\right)$ & $\eta$ & $\left(\frac{W}{m \cdot K}\right)$ & $\left(\frac{M J}{m^{3} \cdot K}\right)$ & $\eta$ & $\left(\frac{W}{m \cdot K}\right)$ & $\left(\frac{M J}{m^{3} \cdot K}\right)$ \\
\hline 0.47 & 0.99 & 1.74 & 0.53 & 0.64 & 1.51 & 0.53 & 0.52 & 1.50 \\
\hline 0.48 & 0.97 & 1.71 & 0.53 & 0.64 & 1.51 & 0.54 & 0.45 & 1.42 \\
\hline 0.50 & 0.92 & 1.56 & 0.56 & 0.47 & 1.38 & 0.56 & 0.40 & 1.31 \\
\hline 0.48 & 0.89 & - & 0.56 & 0.44 & - & 0.51 & 0.71 & - \\
\hline
\end{tabular}

Note: The last row is from Tarnawski et al. [57].
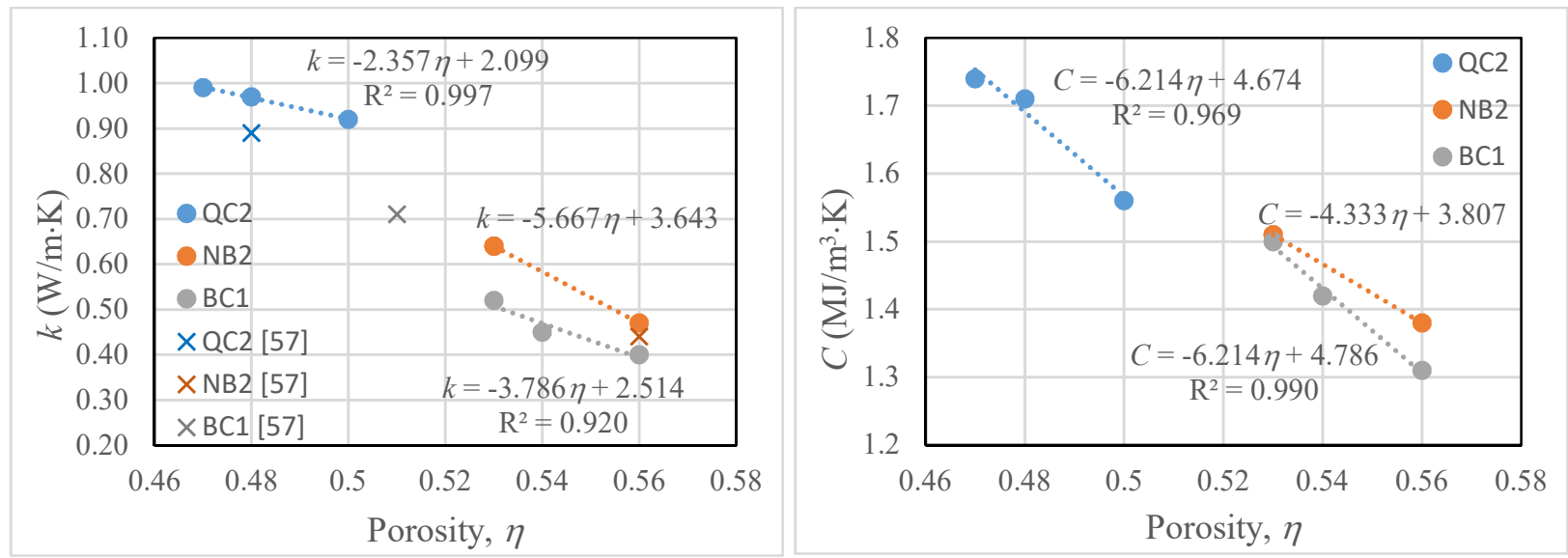

Figure 4.3. Thermal properties of soils $\left(S R \approx 0.25, T \approx 22^{\circ} \mathrm{C}\right)$ with fitted linear relations of porosity and $R^{2}$ values.

\subsection{2 $-S R \approx 0.50$ (Field capacity)}

The measured thermal properties of the wet soils $(\mathrm{SR} \approx 0.50)$ at room temperature are shown in Table 4.9. Similar to soils with $\mathrm{SR} \approx 0.25$, the thermal properties are lower with higher porosity values. Fig. 4.4 shows the thermal properties of NB2 soil at $\mathrm{SR}=0.25$ and 0.50 to illustrate the differences due to different SRs. As the moisture content increases, the thermal properties also increase. It is clear that the thermal properties of soils depend strongly on moisture content or SR. 
Table 4.9. Measured thermal properties of wet soils ( $S R \approx 0.50$, room temperature).

\begin{tabular}{|c|c|c|c|c|c|c|c|c|}
\hline \multicolumn{2}{|c|}{$Q \boldsymbol{C} 2(\boldsymbol{S R} \approx \mathbf{0 . 5 0})$} & \multicolumn{3}{c|}{ NB2 $(\mathbf{S R} \approx \mathbf{0 . 5 0})$} & \multicolumn{3}{c|}{ BC1 (SR $\approx 0.50)$} \\
\hline$\eta$ & $\left(\frac{W}{m \cdot K}\right)$ & $\left(\frac{M J}{m^{3} \cdot K}\right)$ & $\eta$ & $\left(\frac{W}{m \cdot K}\right)$ & $\left(\frac{M J}{m^{3} \cdot K}\right)$ & $\eta$ & $\left(\frac{W}{m \cdot K}\right)$ & $\left(\frac{M J}{m^{3} \cdot K}\right)$ \\
\hline 0.42 & 1.70 & 2.19 & 0.53 & 1.22 & 2.21 & 0.51 & 0.85 & 1.60 \\
\hline 0.44 & 1.63 & 2.13 & 0.55 & 1.13 & 2.07 & 0.51 & 0.83 & 1.61 \\
\hline 0.44 & 1.64 & 2.12 & 0.56 & 1.05 & 1.99 & 0.51 & 0.81 & 1.59 \\
\hline 0.48 & 1.21 & - & 0.56 & 0.88 & - & 0.51 & 0.71 & - \\
\hline
\end{tabular}

Note: The last row is from Tarnawski et al. [57].
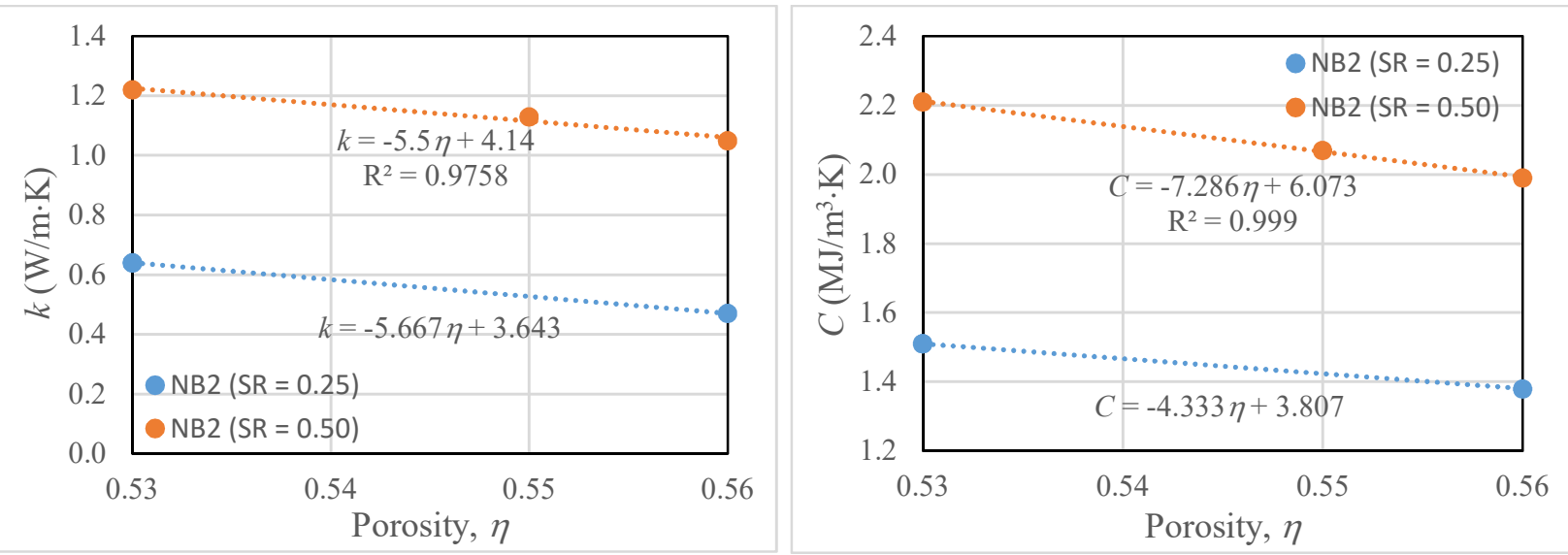

Figure 4.4. Thermal properties of $N B 2$ soil $\left(S R=0.25\right.$ and $\left.0.50, T \approx 22^{\circ} \mathrm{C}\right)$ with fitted linear relations of porosity and $R^{2}$ values.

Table 4.10 shows the fitted coefficients of Eq. 4.8 based on the measured thermal properties of the wet soils $(\mathrm{SR} \approx 0.50)$ as linear functions of temperature. The soils were tested starting from the room temperature of $22^{\circ} \mathrm{C}$ up to the highest tested temperatures (with a heating rate of maximum $2^{\circ} \mathrm{C}$ per hour), as shown in Table 4.10, for each soil with different porosity. Due to the difficulties in soil sample preparation and experimental setup, the soils cannot be exactly prepared to a desired compaction and moisture content. In fact, the porosity is calculated based on the total mass of the wet soil in the soil column. In other words, the soil porosity is determined after a soil is compacted into the soil column. Consequently, there are slight variations in the porosities and thermal properties in Table 4.10 even if the porosity values appear to be the same with two decimal places. 
Table 4.10. Measured thermal properties of wet soils $(S R \approx 0.50)$ as functions of temperature from $22^{\circ} \mathrm{C}$ up to the indicated highest temperature.

\begin{tabular}{|c|c|c|c|c|c|c|c|c|c|c|}
\hline \multirow{2}{*}{\multicolumn{2}{|c|}{$\frac{\text { Soil ID }}{\text { Porosity, } \eta}$}} & \multicolumn{3}{|c|}{$Q C 2$} & \multicolumn{3}{|c|}{ NB2 } & \multicolumn{3}{|c|}{$B C 1$} \\
\hline & & 0.42 & 0.42 & 0.45 & 0.52 & 0.51 & 0.51 & 0.55 & 0.55 & 0.56 \\
\hline \multicolumn{2}{|c|}{ Highest temperature } & $62^{\circ} \mathrm{C}$ & $52^{\circ} \mathrm{C}$ & $42^{\circ} \mathrm{C}$ & $62^{\circ} \mathrm{C}$ & $52^{\circ} \mathrm{C}$ & $42^{\circ} \mathrm{C}$ & $62^{\circ} \mathrm{C}$ & $52^{\circ} \mathrm{C}$ & $42^{\circ} \mathrm{C}$ \\
\hline \multirow{3}{*}{$\left.\begin{array}{c}k \\
W \\
m \cdot K\end{array}\right)$} & $a_{X}$ & 139 & 86.8 & 117 & 165 & 141 & 219 & 144 & 139 & 116 \\
\hline & $b_{X}$ & 1.34 & 1.49 & 1.54 & 0.77 & 0.89 & 0.66 & 0.49 & 0.44 & 0.52 \\
\hline & $R^{2}$ & 0.925 & 0.923 & 0.922 & 0.933 & 0.930 & 0.931 & 0.930 & 0.932 & 0.926 \\
\hline \multirow{3}{*}{$\left.\begin{array}{c}C \\
M J \\
n^{3} \cdot K\end{array}\right)$} & $a_{X}$ & 22.2 & 31.3 & 61.2 & 6.45 & 10.3 & 47.3 & 81.2 & 63.9 & 16.6 \\
\hline & $b_{X}$ & 2.10 & 2.06 & 1.94 & 2.19 & 2.22 & 1.86 & 2.09 & 2.10 & 2.09 \\
\hline & $R^{2}$ & 0.960 & 0.959 & 0.961 & 0.957 & 0.955 & 0.958 & 0.956 & 0.957 & 0.953 \\
\hline
\end{tabular}

Further experimentation with $\mathrm{NB} 2$ soil $(\mathrm{SR} \approx 0.50, \eta \approx 0.51)$ shows that the thermal properties increase linearly with higher temperatures up to $90^{\circ} \mathrm{C}$, as shown in Table 4.11 . The fitted coefficients in Table 4.11 are similar to the third column under NB2 from $22^{\circ} \mathrm{C}$ to $42^{\circ} \mathrm{C}$ in Table 4.10. Because of non-uniform moisture distribution of the wet soil after experimentation (moisture migration due to gravity only at temperatures above $40^{\circ} \mathrm{C}$ ), a wet soil sample is prepared and compacted for each experiment. Due to difficulties in sample preparations and compaction into the soil column, the NB2 soil in Table 4.11 is not the same as that in Table 4.10. Nonetheless, Fig. 4.5 shows the three fitted linear relations of NB2 soil thermal properties over their respective ranges of temperature from Tables 4.10 and 4.11 for the case of $\eta \approx 0.51$. It can be seen that the values are quite close to each other. The differences for thermal conductivity with respect to the average values are within $\pm 7 \%$, while the differences for volumetric heat capacity are within $\pm 8 \%$. For one NB2 soil sample tested from $22^{\circ} \mathrm{C}$ to $52^{\circ} \mathrm{C}$ (with solid line in Fig. 4.5), its slope is significantly different from the other two samples. So, if it is used to estimate the thermal properties outside the temperature range, the properties will be significantly different from the other two samples. Therefore, it is recommended to only use the relations within their valid temperature ranges. 
Table 4.11. Fitted coefficients of Eq. 4.8 for $k$ and $C$ of a wet NB2 soil $(S R \approx 0.50, \eta \approx 0.51$, $\left.22{ }^{\circ} \mathrm{C} \leq T \leq 90{ }^{\circ} \mathrm{C}\right)$.

\begin{tabular}{|c|c|c|c|c|c|}
\hline \multirow{3}{*}{$\left(\frac{W}{m \cdot K}\right)$} & $a_{X}$ & 210.7 & & $a_{X}$ & 58.1 \\
\cline { 2 - 3 } \cline { 5 - 5 } & $b_{X}$ & 0.561 & $\left(\frac{M J}{m^{3} \cdot K}\right)$ & $b_{X}$ & 1.89 \\
\cline { 2 - 3 } \cline { 5 - 5 } & $R^{2}$ & 0.921 & & $R^{2}$ & 0.943 \\
\hline
\end{tabular}
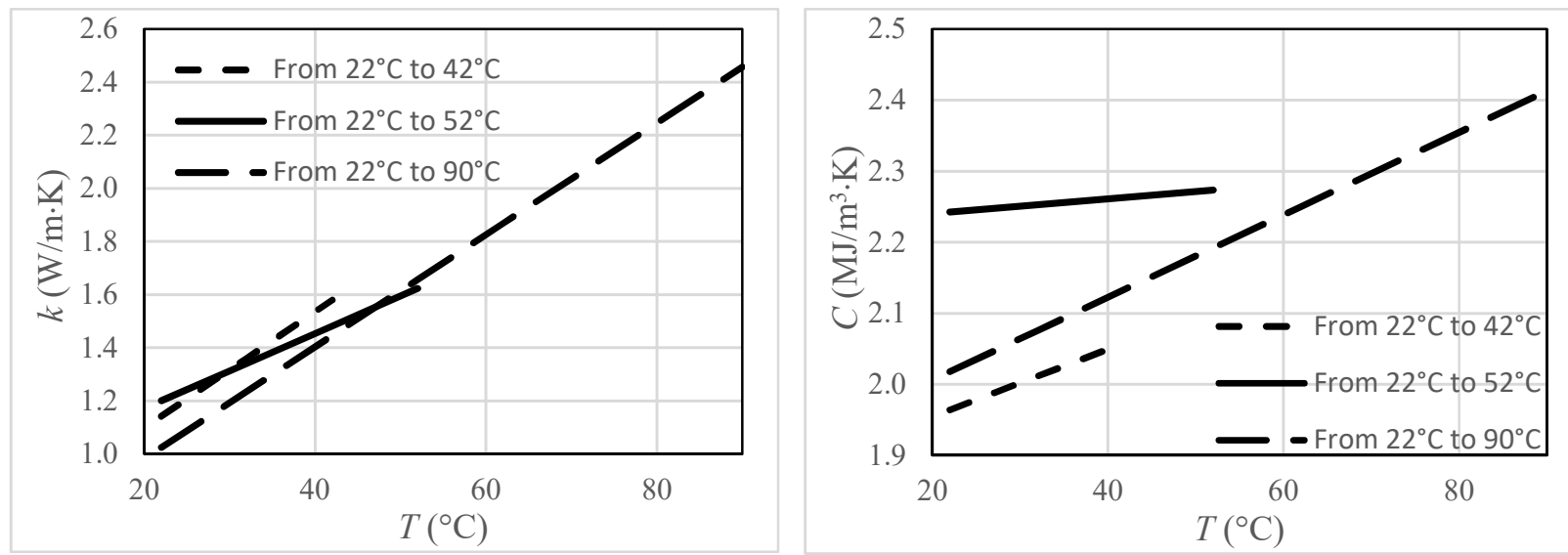

Figure 4.5. Fitted linear relations of thermal properties of $N B 2$ soil $(S R \approx 0.50, \eta \approx 0.51)$ for three different soil samples and temperature ranges.

\section{$4.3 .3-S R \approx 0.70$}

Table 4.12 shows the measured thermal properties of wet QC2 and NB2 soils $(\mathrm{SR} \approx 0.7)$ at $22^{\circ} \mathrm{C}$ and $90^{\circ} \mathrm{C}$. Due to the difficulty in preparing $\mathrm{BC} 1$ soil at high $\mathrm{SR}, \mathrm{BC} 1$ soil is not tested for $\mathrm{SR} \approx 0.7$. Table 4.13 shows the fitted coefficients of Eq. 4.8 for the measured thermal properties of wet $\mathrm{QC} 2$ and $\mathrm{NB} 2$ soils $(\mathrm{SR} \approx 0.7)$ from $22^{\circ} \mathrm{C}$ to $90^{\circ} \mathrm{C}$. It is interesting to see that the thermal conductivity of QC2 soil increases by $44 \%$ from $22^{\circ} \mathrm{C}$ to $90^{\circ} \mathrm{C}$, while its volumetric heat capacity only increases by about $12 \%$. However, the thermal conductivity of NB2 soil increases by about $53 \%$ over the same temperature range, while its volumetric heat capacity only increases by about $2 \%$. This indicates that the temperature has much bigger effect on the thermal conductivity than on the volumetric heat capacity.

From Table 4.13, the positive values of $a_{X}$ indicate that the properties of the soils increase linearly with higher temperatures. Comparing with the thermal conductivities of the soils with the ones from Tarnawski et al. [57] at the room temperature $\left(\approx 22^{\circ} \mathrm{C}\right)$, Table 4.12 shows higher values 
of $k$ ( $14.4 \%$ for QC2 and $17.5 \%$ for NB2). The differences can be attributable to the three reasons discussed in section 4.3.1.

Table 4.12. Measured thermal properties of wet soils $(S R \approx 0.7)$

\begin{tabular}{|c|c|c|c|c|c|c|c|c|c|c|c|}
\hline \multicolumn{3}{|c|}{$\boldsymbol{Q C 2}\left(\boldsymbol{T}=\mathbf{2 2}^{\circ} \mathrm{C}\right)$} & \multicolumn{3}{c|}{$\boldsymbol{Q C 2}\left(\boldsymbol{T}=\mathbf{9 0}^{\circ} \mathrm{C}\right)$} & \multicolumn{3}{c|}{ NB2 $\left(\boldsymbol{T}=22^{\circ} \boldsymbol{C}\right)$} & \multicolumn{3}{c|}{$\boldsymbol{N B 2}\left(\boldsymbol{T}=\mathbf{9 0}{ }^{\circ} \mathrm{C}\right)$} \\
\hline$\eta$ & $k$ & $C$ & $\eta$ & $k$ & $C$ & $\eta$ & $k$ & $C$ & $\eta$ & $k$ & $C$ \\
\hline 0.40 & 1.52 & 2.63 & 0.40 & 2.19 & 2.94 & 0.51 & 1.37 & 2.70 & 0.51 & 2.09 & 2.74 \\
\hline 0.48 & 1.37 & - & - & - & - & 0.56 & 1.19 & - & - & - & - \\
\hline
\end{tabular}

Note: The last row is from Tarnawski et al. [57]. $k$ is in $W / m \cdot \mathrm{K}$ and $C$ is in $M J / m^{3} \cdot \mathrm{K}$.

Table 4.13. Measured thermal properties of wet soils $(S R \approx 0.70)$ as functions of temperature from $22^{\circ} \mathrm{C}$ to $90^{\circ} \mathrm{C}$.

\begin{tabular}{|c|c|c|c|c|c|c|c|}
\hline \multirow{2}{*}{$\begin{array}{c}\text { Soil } \\
\text { ID }\end{array}$} & \multicolumn{3}{|c|}{$\left(\frac{1}{m \cdot K}\right)$} & \multicolumn{3}{c|}{$\left(\frac{M J}{m^{3} \cdot K}\right)$} & \multirow{2}{*}{$\eta$} \\
\cline { 2 - 7 } & $a_{X}$ & $b_{X}$ & $R^{2}$ & $a_{X}$ & $b_{X}$ & $R^{2}$ & \\
\hline QC2 & 94.0 & 1.36 & 0.921 & 42.5 & 2.57 & 0.948 & 0.42 \\
\hline NB2 & 104.0 & 1.17 & 0.923 & 2.39 & 2.73 & 0.945 & 0.51 \\
\hline
\end{tabular}

\section{4 - More about Measured Thermal Properties of Soils}

In this section, measured thermal properties of the tested soils are explored more. Fig. 4.6 shows the values of the thermal properties from different porosities of each soil type for three tested soil types. As the moisture content is higher (i.e., higher SR), the values of the thermal properties are higher. In addition, when the temperature is higher, the thermal properties have higher values. Moreover, since water has a high thermal capacity, the heat capacities of the three tested soils become closer to each other with higher moisture content. On the other hand, water's and sand's thermal conductivities are higher than those of silt and clay. Consequently, when the soil has higher moisture content, the medium and fine soils' thermal conductivities nonlinearly increase in terms of SR. Furthermore, dry QC2 has lower thermal conductivity and heat capacity than water does as shown in Tables $4.3-4.5$, so the addition of moisture significantly affects 
QC2's thermal conductivity and heat capacity. Due to the time restraint, the three soil types with $\mathrm{SR} \approx 0.25$ were not tested with gradual heating (i.e., the soil's temperature is increased $2^{\circ} \mathrm{C}$ per hour). As a result, the two bottom graphs of Fig. 4.6 do not show any thermal property value for $\mathrm{SR}=0.25$. The reader should take considerations in linear-interpolating the lines in the bottom graphs of Fig. 4.6.
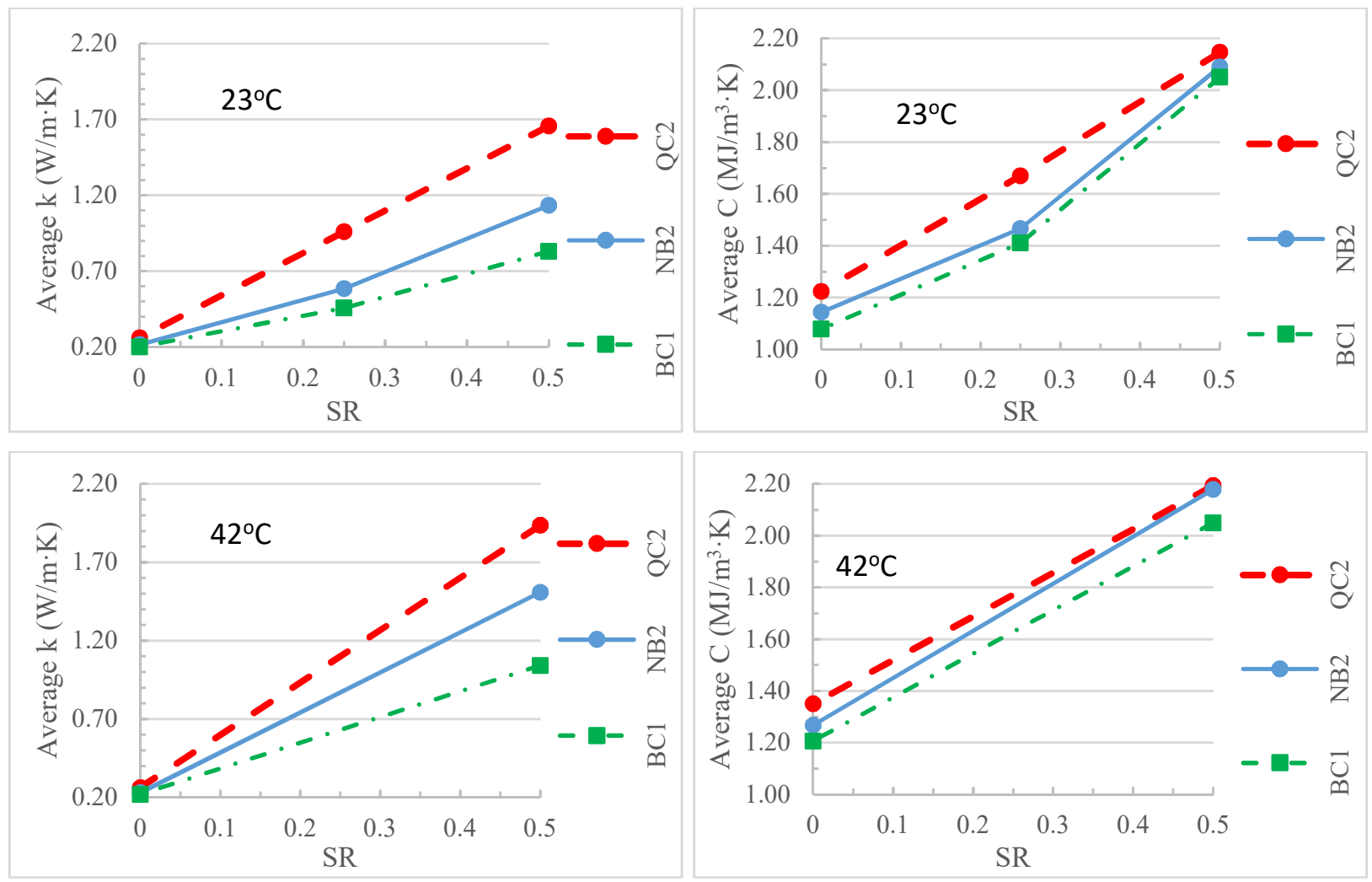

Figure 4.6. Average thermal properties of tested soils at different temperatures.

\section{5 - Thermal Storage Behaviors of Tested Soils}

In this thesis, there are 27 cases ( 9 cases for dry soils and 18 cases for wet soils) that have large temperature differences between the hot and cold plates $\left(90^{\circ} \mathrm{C}\right.$ at the hot plate and $10^{\circ} \mathrm{C}$ at the cold plate) of the soil cell. The times for the soils to reach $95 \%$ temperatures at the steady-state conditions are shown in Table 4.14. As shown in Table 4.14, the drier soils take longer to heat up 
because they have lower thermal conductivities than the wetter soils do, even though the drier soils have lower heat capacitances.

Table 4.14. Times to reach 95\% temperatures at steady-state conditions for the tested soils

\begin{tabular}{|c|c|c|c|}
\hline SR & 0.00 & 0.25 & 0.50 \\
\hline Time & 3.00 hours & 2.50 hours & 2.25 hours \\
\hline
\end{tabular}

\section{6 - Summary}

In this chapter, uncertainty analysis and measured thermal properties of soils were shown. Due to the difficulties in soil preparation and heat pulse probe construction, the overall uncertainties of the thermal properties appeared slightly high with the maximum values of $15.5 \%$ and $9.18 \%$ for $k$ and $C$ respectively. The uncertainties for moisture content were higher for less moist soils, and the maximum uncertainties were $48.6 \%$ and $29.9 \%$ for measuring moisture content in soils with $\mathrm{SR}=0.25$ and 0.5 , respectively.

Thermal properties $(k$ and $C$ ) of the soils were tabulated. The porosities were very difficult to be predetermined, so they were calculated after the soil was compacted (layer by layer) into the soil column. Even though the porosity values appeared the same in some tables, the measured thermal properties were quite different; nonetheless, their variations were within the maximum overall uncertainties. In addition, there were slight variations in the heat pulse probes, soil compaction procedures, and water baths' performances. As a result, there were different fitted coefficients of thermal properties as linear functions of temperature. In general, the thermal properties increase linearly with temperatures.

The values of thermal conductivities were also shown to be higher than those from Tarnawski et al. [57]. In some cases, the difference can be as much as 30\%. There are three reasons that may contribute to the differences, and they are discussed in Section 4.3.1.

Next chapter will show the one-dimensional and axisymmetric formulations using the finite volume method (FVM) to numerically study the heat and moisture simulations in soils. 


\section{CHAPTER 5 - NUMERICAL FORMULATIONS USING FINITE VOLUME METHOD}

\section{1 - Introduction}

The governing equations of heat and moisture transfer in soils by Deru [28] (i.e., Eq. 2.5) contained coupled heat and moisture terms. Since there is no analytical solution to the equations, numerical methods are used to solve the equations. The finite volume method (FVM) is used to numerically study the governing equations because the method guarantees energy conservation.

\section{2 - Numerical Solution}

\subsection{1 - Finite Volume Method - One-Dimensional Formulation}

In this section, one-dimensional (1D) formulations of the finite volume method will be developed as shown in Fig. 5.1 with the following assumptions:

- The height of the soil column is $h$ (distance from node A to node B)

- There are $n$ control volumes (CVs) along $h$

- Each CV has length $\Delta z$ of $h / n$

- The distance from node $\mathrm{A}$ to node 1 is the same as that from $n$ to $\mathrm{B}$ and is equal to $\Delta z / 2$

- There are $n+2$ nodes

- The temperatures at nodes $\mathrm{A}$ and $\mathrm{B}$ are known at all times 


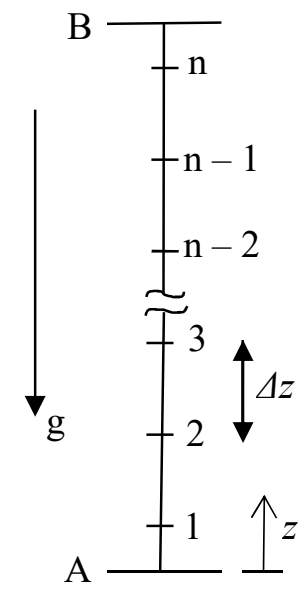

Figure 5.1. One-dimensional discretization scheme

In order to solve the governing equations, the CVs are added together as follows:

$$
\begin{aligned}
\int_{t}^{t+\Delta t} \int_{C V}\left(C_{\psi m} \frac{\partial \psi}{\partial t}+C_{T m} \frac{\partial T}{\partial t}\right) d \forall d t & =\int_{t}^{t+\Delta t} \int_{C V}\left[\frac{\partial}{\partial z}\left(D_{\psi m} \frac{\partial \psi}{\partial z}\right)\right] d \forall d t+\int_{t}^{t+\Delta t} \int_{C V}\left[\frac{\partial}{\partial z}\left(D_{T m} \frac{\partial T}{\partial z}\right)\right] d \forall d t \\
& +\int_{t}^{t+\Delta t} \int_{C V} \frac{\partial K}{\partial z} d \forall d t
\end{aligned}
$$

$\int_{t}^{t+\Delta t} \int_{C V}\left(C_{\psi T} \frac{\partial \psi}{\partial t}+C_{T T} \frac{\partial T}{\partial t}\right) d \forall d t=\int_{t}^{t+\Delta t} \int_{C V}\left[\frac{\partial}{\partial z}\left(D_{\psi T} \frac{\partial \psi}{\partial z}\right)\right] d \forall d t+\int_{t}^{t+\Delta t} \int_{C V}\left[\frac{\partial}{\partial z}\left(k_{e f f} \frac{\partial T}{\partial z}\right)\right] d \forall d t$

where $d \forall$ is the volume of the CV.

Applying $d \forall=A \cdot \Delta z$ with $A$ being the soil's column cross-section area, Eq. 5.1 can be written as follow:

$$
\begin{aligned}
\frac{\Delta z}{\Delta t}\left[C_{\psi m}\left(\psi_{p}-\psi_{p}^{o}\right)+C_{T m}\left(T_{p}-T_{p}^{o}\right)\right]= & \beta\left[\left(D_{\psi m}\right)_{n} \frac{\psi_{N}-\psi_{P}}{\Delta z}-\left(D_{\psi m}\right)_{s} \frac{\psi_{P}-\psi_{S}}{\Delta z}\right] \\
& +(1-\beta)\left[\left(D_{\psi m}\right)_{n}^{o} \frac{\psi_{N}^{o}-\psi_{P}^{o}}{\Delta z}-\left(D_{\psi m}\right)_{s}^{o} \frac{\psi_{P}^{o}-\psi_{S}^{o}}{\Delta z}\right] \\
& +\beta\left[\left(D_{T m}\right)_{n} \frac{T_{N}-T_{P}}{\Delta z}-\left(D_{T m}\right)_{s} \frac{T_{P}-T_{S}}{\Delta z}\right] \\
& +(1-\beta)\left[\left(D_{T m}\right)_{n}^{o} \frac{T_{N}^{o}-T_{P}^{o}}{\Delta z}-\left(D_{T m}\right)_{s}^{o} \frac{T_{P}^{o}-T_{S}^{o}}{\Delta z}\right] \\
& +\beta\left[(K)_{n}-(K)_{s}\right]+(1-\beta)\left[(K)_{n}^{o}-(K)_{s}^{o}\right]
\end{aligned}
$$




$$
\begin{aligned}
\frac{\Delta z}{\Delta t}\left[C_{\psi T}\left(\psi_{p}-\psi_{p}^{o}\right)+C_{T T}\left(T_{p}-T_{p}^{o}\right)\right]= & \beta\left[\left(D_{\psi T}\right)_{n} \frac{\psi_{N}-\psi_{P}}{\Delta z}-\left(D_{\psi T}\right)_{s} \frac{\psi_{P}-\psi_{S}}{\Delta z}\right] \\
& +(1-\beta)\left[\left(D_{\psi T}\right)_{n}^{o} \frac{\psi_{N}^{o}-\psi_{P}^{o}}{\Delta z}-\left(D_{\psi T}\right)_{s}^{o} \frac{\psi_{P}^{o}-\psi_{S}^{o}}{\Delta z}\right] \\
& +\beta\left[\left(k_{\text {eff }}\right)_{n} \frac{T_{N}-T_{P}}{\Delta z}-\left(k_{\text {eff }}\right)_{s} \frac{T_{P}-T_{S}}{\Delta z}\right] \\
& +(1-\beta)\left[\left(k_{e f f}\right)_{n}^{o} \frac{T_{N}^{o}-T_{P}^{o}}{\Delta z}-\left(k_{e f f}\right)_{s}^{o} \frac{T_{P}^{o}-T_{S}^{o}}{\Delta z}\right]
\end{aligned}
$$

where the superscript ${ }^{\circ}$ means the value at previous time step, $\mathrm{P}$ means the centroid of the current $\mathrm{CV}$ being considered, $\mathrm{N}$ means the north of the CV's centroid, $\mathrm{S}$ means the south of the CV's centroid, and $\beta$ is the factor that switches from explicit to implicit iteration or vice versa. $\beta=0$ : explicit. $\beta=1$ : implicit. $\beta=0.5$ : similar to Crank-Nicolson's method. Diffusion coefficients are evaluated at the average values ( $\psi_{\text {avg }}$ and $T_{a v g}$ ) of the two nearest nodes [60]. This thesis uses $\beta=$ 0.5 for better stability and faster iterations.

Re-arranging Eq. 5.2, the discretized governing equations for nodes 2 to $n-1$ are:

$$
\begin{gathered}
a_{1} \psi_{P}+a_{2} T_{P}=a_{3} \psi_{N}+a_{4} T_{N}+a_{5} \psi_{S}+a_{6} T_{S}+G_{1} \\
b_{1} \psi_{P}+b_{2} T_{P}=b_{3} \psi_{N}+b_{4} T_{N}+b_{5} \psi_{S}+b_{6} T_{S}+G_{2}
\end{gathered}
$$

where $a_{1}=C_{\psi m}+a_{3}+a_{5}, \quad a_{2}=C_{T m}+a_{4}+a_{6}, \quad b_{1}=C_{\psi T}+b_{3}+b_{5}, \quad b_{2}=C_{T T}+b_{4}+b_{6}$,

$$
\begin{gathered}
a_{3}=\frac{\beta \Delta t}{(\Delta z)^{2}}\left(D_{\psi m}\right)_{n}, \quad a_{4}=\frac{\beta \Delta t}{(\Delta z)^{2}}\left(D_{T m}\right)_{n}, \quad a_{5}=\frac{\beta \Delta t}{(\Delta z)^{2}}\left(D_{\psi m}\right)_{s}, \quad a_{6}=\frac{\beta \Delta t}{(\Delta z)^{2}}\left(D_{T m}\right)_{s}, \\
b_{3}=\frac{\beta \Delta t}{(\Delta z)^{2}}\left(D_{\psi T}\right)_{n}, \quad b_{4}=\frac{\beta \Delta t}{(\Delta z)^{2}}\left(k_{e f f}\right)_{n}, \quad b_{5}=\frac{\beta \Delta t}{(\Delta z)^{2}}\left(D_{\psi T}\right)_{s}, \quad b_{6}=\frac{\beta \Delta t}{(\Delta z)^{2}}\left(k_{e f f}\right)_{s}, \\
G_{1}=\left\{C_{\psi m}-\frac{(1-\beta)}{\beta}\left[a_{3}^{o}+a_{5}^{o}\right]\right\} \psi_{P}^{o}+\left\{C_{T m}-\frac{(1-\beta)}{\beta}\left[a_{4}^{o}+a_{6}^{o}\right]\right\} T_{P}^{o} \\
+\frac{(1-\beta)}{\beta}\left\{a_{3}^{o} \psi_{N}^{o}+a_{5}^{o} \psi_{S}^{o}+a_{4}^{o} T_{N}^{o}+a_{6}^{o} T_{S}^{o}+\Delta z\left(K_{n}^{o}-K_{s}^{o}\right)\right\}+\frac{\beta \Delta t}{\Delta z}\left\{K_{n}-K_{s}\right\}
\end{gathered}
$$




$$
G_{2}=\left\{C_{\psi T}-\frac{(1-\beta)}{\beta}\left[b_{3}^{o}+b_{5}^{o}\right]\right\} \psi_{P}^{o}+\left\{C_{T T}-\frac{(1-\beta)}{\beta}\left[b_{4}^{o}+b_{6}^{o}\right]\right\} T_{P}^{o}+\frac{(1-\beta)}{\beta}\left\{b_{3}^{o} \psi_{N}^{o}+b_{5}^{o} \psi_{S}^{o}+b_{4}^{o} T_{N}^{o}+b_{6}^{o} T_{S}^{o}\right\}
$$

\section{Boundary conditions:}

The temperatures at nodes $\mathrm{A}$ and $\mathrm{B}\left(T_{A}\right.$ and $\left.T_{B}\right)$ are measured in the experiment and therefore they are used as prescribed boundary temperatures. According to Deru [28], the moisture balance at nodes A and B, for impermeable surfaces due to the stainless-steel lids, is:

$$
0=-K \frac{\partial \Phi}{\partial n}-D_{\psi v} \frac{\partial \psi}{\partial n}-D_{T m} \frac{\partial T}{\partial n}
$$

where $\Phi=\psi+z$ with $z$ being positive upwards $(z=0$ at node A) and $\partial / \partial n=\partial / \partial z$.

As a result of Eq. 5.4, matric potential gradients at nodes A and B respectively are:

$$
\begin{aligned}
& \left.\frac{\partial \psi}{\partial z}\right|_{A}=\frac{-1}{K+D_{\psi v}}\left(\left.D_{T m} \frac{\partial T}{\partial z}\right|_{z=A}+K\right) \\
& \left.\frac{\partial \psi}{\partial z}\right|_{B}=\frac{-1}{K+D_{\psi v}}\left(\left.D_{T m} \frac{\partial T}{\partial z}\right|_{z=B}+K\right)
\end{aligned}
$$

Applying the boundary conditions (Eqs. 5.2 and 5.5a) at node 1, we have:

$$
\begin{aligned}
\frac{\Delta z}{\Delta t}\left[C_{\psi m}\left(\psi_{1}-\psi_{1}^{o}\right)+C_{T m}\left(T_{1}-T_{1}^{o}\right)\right] \\
=\beta\left[\left(D_{\psi m}\right)_{2} \frac{\psi_{2}-\psi_{1}}{\Delta z}-\left(\frac{-D_{\psi m}}{K+D_{\psi v}}\right)_{1}\left(K_{s}+\left(D_{\psi m}\right)_{1} \frac{T_{1}-T_{A}}{\Delta z / 2}\right)\right] \\
\quad+(1-\beta)\left[\left(D_{\psi m}\right)_{2}^{o} \frac{\psi_{2}^{o}-\psi_{1}^{o}}{\Delta z}-\left(\frac{-D_{\psi m}}{K+D_{\psi v}}\right)_{1}^{o}\left(K_{1}^{o}+\left(D_{\psi m}\right)_{1}^{o} \frac{T_{1}^{o}-T_{A}^{o}}{\Delta z / 2}\right)\right] \\
+\beta\left[\left(D_{T m}\right)_{2} \frac{T_{2}-T_{1}}{\Delta z}-\left(D_{T m}\right)_{1} \frac{T_{1}-T_{A}}{\Delta z / 2}\right] \\
\quad+(1-\beta)\left[\left(D_{T m}\right)_{1}^{o} \frac{T_{2}^{o}-T_{1}^{o}}{\Delta z}-\left(D_{T m}\right)_{1}^{o} \frac{T_{1}^{o}-T_{A}^{o}}{\Delta z / 2}\right] \\
\quad+\beta\left[(K)_{2}-(K)_{1}\right]+(1-\beta)\left[(K)_{2}^{o}-(K)_{1}^{o}\right]
\end{aligned}
$$




$$
\begin{aligned}
& \frac{\Delta z}{\Delta t}\left[C_{\psi T}\left(\psi_{1}-\psi_{1}^{o}\right)+C_{T T}\left(T_{1}-T_{1}^{o}\right)\right] \\
& =\beta\left[\left(D_{\psi T}\right)_{2} \frac{\psi_{2}-\psi_{1}}{\Delta z}-\left(\frac{D_{T m}}{K+D_{\psi v}}\right)_{1}\left(K_{1}+\left(D_{\psi T}\right)_{1} \frac{T_{1}-T_{A}}{\Delta z / 2}\right)\right] \\
& \quad+(1-\beta)\left[\left(D_{\psi T}\right)_{2}^{o} \frac{\psi_{2}^{o}-\psi_{1}^{o}}{\Delta z}-\left(\frac{D_{T m}}{K+D_{\psi v}}\right)_{1}^{o}\left(K_{1}^{o}+\left(D_{\psi T}\right)_{1}^{o} \frac{T_{1}^{o}-T_{A}^{o}}{\Delta z / 2}\right)\right] \\
& \quad+\beta\left[\left(k_{e f f}\right)_{2} \frac{T_{2}-T_{1}}{\Delta z}-\left(k_{e f f}\right)_{1} \frac{T_{1}-T_{A}}{\Delta z / 2}\right] \\
& \quad+(1-\beta)\left[\left(k_{e f f}\right)_{2}^{o} \frac{T_{2}^{o}-T_{1}^{o}}{\Delta z}-\left(k_{e f f}\right)_{1}^{o} \frac{T_{1}^{o}-T_{A}^{o}}{\Delta z / 2}\right]
\end{aligned}
$$

Re-arranging Eq. 5.6, the discretized governing equations at node 1 are:

$$
\begin{aligned}
& a_{1} \psi_{1}+a_{2} T_{1}=a_{3} \psi_{2}+a_{4} T_{2}+a_{5} \psi_{A}+a_{6} T_{A}+G_{1} \\
& b_{1} \psi_{1}+b_{2} T_{1}=b_{3} \psi_{2}+b_{4} T_{2}+b_{5} \psi_{A}+b_{6} T_{A}+G_{2}
\end{aligned}
$$

where $\quad a_{1}=C_{\psi m}+a_{3}+a_{5}, \quad a_{2}=C_{T m}+a_{4}+a_{6}, \quad b_{1}=C_{\psi T}+b_{3}+b_{5}, \quad b_{2}=C_{T T}+b_{4}+b_{6}$,

$$
\begin{aligned}
a_{3}= & \frac{\beta \Delta t}{(\Delta z)^{2}}\left(D_{\psi m}\right)_{2}, \quad a_{4}=\frac{\beta \Delta t}{(\Delta z)^{2}}\left(D_{T m}\right)_{2}, \quad a_{5}=0, \quad a_{6}=\frac{2 \beta \Delta t}{(\Delta z)^{2}}\left[\left(D_{T m}\right)_{1}+\left(\Lambda_{a}\right)_{1}\right] \\
b_{3}= & \frac{\beta \Delta t}{(\Delta z)^{2}}\left(D_{\psi T}\right)_{2}, \quad b_{4}=\frac{\beta \Delta t}{(\Delta z)^{2}}\left(k_{e f f}\right)_{2}, \quad b_{5}=0, \quad b_{6}=\frac{2 \beta \Delta t}{(\Delta z)^{2}}\left[\left(k_{e f f}\right)_{1}+\left(\Lambda_{b}\right)_{1}\right], \\
G_{1}= & \left\{C_{\psi m}-\frac{(1-\beta)}{\beta} a_{3}^{o}\right\} \psi_{1}^{o}+\left\{C_{T m}-\frac{(1-\beta)}{\beta}\left[a_{4}^{o}+a_{6}^{o}\right]\right\} T_{1}^{o}+\frac{\beta \Delta t}{\Delta z}\left\{K_{2}-K_{1}+\left(\frac{\Lambda_{a} K}{D_{T m}}\right)\right\} \\
& +\frac{(1-\beta)}{\beta}\left\{a_{3}^{o} \psi_{2}^{o}+a_{4}^{o} T_{2}^{o}+a_{6}^{o} T_{A}^{o}+\frac{\beta \Delta t}{\Delta z}\left(K_{2}^{o}-K_{1}^{o}+\left(\frac{\Lambda_{b} K}{D_{T m}}\right)_{1}^{o}\right)\right\} \\
G_{2}= & \left\{C_{\psi T}-\frac{(1-\beta)}{\beta} b_{3}^{o}\right\} \psi_{1}^{o}+\left\{C_{T T}-\frac{(1-\beta)}{\beta}\left[b_{4}^{o}+b_{6}^{o}\right]\right\} T_{1}^{o}+\frac{(1-\beta)}{\beta}\left\{b_{3}^{o} \psi_{2}^{o}+b_{4}^{o} T_{2}^{o}+b_{6}^{o} T_{A}^{o}\right\},
\end{aligned}
$$




$$
\begin{aligned}
& \Lambda_{a}=\left(\frac{-D_{T m} \cdot D_{\psi m}}{K+D_{\psi v}}\right)_{1}, \quad \Lambda_{b}=\left(\frac{-D_{T m} \cdot D_{\psi T}}{K+D_{\psi v}}\right)_{1}, \quad K_{1} \text { means } K \text { at node } 1, \text { and } \\
& \left(D_{x x}\right)_{1} \text { and }\left(k_{e f f}\right)_{1} \text { mean } D_{x x} \text { and } k_{e f f} \text { of CV } 1 \text { respectively }
\end{aligned}
$$

Applying the boundary conditions (Eqs. 5.2 and 5.5b) at node $n$, we have:

$$
\begin{aligned}
\frac{\Delta z}{\Delta t} & {\left[C_{\psi m}\left(\psi_{n}-\psi_{n}^{o}\right)_{+} C_{T m}\left(T_{n}-T_{n}^{o}\right)\right] } \\
& =\beta\left[\left(\frac{-D_{\psi m}}{K+D_{\psi v}}\right)_{n}\left(\left(D_{T m}\right)_{n} \frac{T_{B}-T_{n}}{\Delta z / 2}+K_{n}\right)-\left(D_{\psi m}\right)_{s} \frac{\psi_{n}-\psi_{n-1}}{\Delta z}\right] \\
& +(1-\beta)\left[\left(\frac{-D_{\psi m}}{K+D_{\psi v}}\right)_{n}^{o}\left(\left(D_{T m}\right)_{n} \frac{T_{B}^{o}-T_{n}^{o}}{\Delta z / 2}+K_{n}^{o}\right)-\left(D_{\psi m}\right)_{s}^{o} \frac{\psi_{n}^{o}-\psi_{n-1}^{o}}{\Delta z}\right] \\
& +\beta\left[\left(D_{T m}\right)_{n} \frac{T_{B}-T_{n}}{\Delta z / 2}-\left(D_{T m}\right)_{s} \frac{T_{n}-T_{n-1}}{\Delta z}\right] \\
& +(1-\beta)\left[\left(D_{T m}\right)_{n}^{o} \frac{T_{B}^{o}-T_{n}^{o}}{\Delta z / 2}-\left(D_{T m}\right)_{s}^{o} \frac{T_{n}^{o}-T_{n-1}^{o}}{\Delta z}\right] \\
& +\beta\left[(K)_{n}-(K)_{s}\right]_{+}(1-\beta)\left[(K)_{n}^{o}-(K)_{s}^{o}\right] \\
\frac{\Delta z}{\Delta t} & {\left[C_{\psi T}\left(\psi n-\psi_{n}^{o}\right)_{+}+C_{T T}\left(T_{n}-T_{n}^{o}\right)\right]_{=} } \\
& \quad\left[\left(\frac{-D_{\psi T}}{K+D_{\psi v}}\right)_{n}\left(\left(D_{T m}\right)_{n} \frac{T_{B}-T_{n}}{\Delta z / 2}+K_{n}\right)-\left(D_{\psi T}\right)_{s} \frac{\psi_{P}-\psi_{S}}{\Delta z}\right] \\
& +(1-\beta)\left[\left(\frac{-D_{\psi T}}{K+D_{\psi v}}\right)_{n}^{o}\left(\left(D_{T m}\right)_{n}^{o} \frac{T_{B}^{o}-T_{n}^{o}}{\Delta z / 2}+K_{n}^{o}\right)-\left(D_{\psi T}\right)_{s}^{o} \frac{\psi_{P}^{o}-\psi_{S}^{o}}{\Delta z}\right] \\
& +\beta\left[\left(k_{e f f}\right)_{n} \frac{T_{B}-T_{n}}{\Delta z / 2}-\left(k_{e f f}\right)_{s} \frac{T_{n}-T_{n-1}}{\Delta z}\right] \\
& +(1-\beta)\left[\left(k_{e f f}^{o}\right)_{n}^{o} \frac{T_{B}^{o}-T_{n}^{o}}{\Delta z / 2}-\left(k_{e f f}\right)_{s}^{o} \frac{T_{n}^{o}-T_{n-1}^{o}}{\Delta z}\right]
\end{aligned}
$$

Re-arranging Eq. 5.8b, the discretized governing equations at node $n$ are:

$$
a_{1} \psi_{n}+a_{2} T_{n}=a_{3} \psi_{B}+a_{4} T_{B}+a_{5} \psi_{n-1}+a_{6} T_{n-1}+G_{1}
$$




$$
b_{1} \psi_{n}+b_{2} T_{n}=b_{3} \psi_{B}+b_{4} T_{B}+b_{5} \psi_{n-1}+b_{6} T_{n-1}+G_{2}
$$

where $\quad a_{1}=C_{\psi m}+a_{3}+a_{5}, \quad a_{2}=C_{T m}+a_{4}+a_{6}, \quad b_{1}=C_{\psi T}+b_{3}+b_{5}, \quad b_{2}=C_{T T}+b_{4}+b_{6}$,

$$
\begin{gathered}
a_{3}=0, \quad a_{4}=\frac{\beta \Delta t}{(\Delta z)^{2}}\left(D_{T m}\right)_{n-1}, \quad a_{5}=\frac{\beta \Delta t}{(\Delta z)^{2}}\left(D_{\psi m}\right)_{n-1}, \quad a_{6}=\frac{2 \beta \Delta t}{(\Delta z)^{2}}\left[\left(D_{T m}\right)_{n}+\left(\Lambda_{c}\right)_{n}\right], \\
b_{3}=0, \quad b_{4}=\frac{\beta \Delta t}{(\Delta z)^{2}}\left(k_{e f f}\right)_{n-1}, \quad b_{5}=\frac{\beta \Delta t}{(\Delta z)^{2}}\left(D_{\psi T}\right)_{n-1}, \quad b_{6}=\frac{2 \beta \Delta t}{(\Delta z)^{2}}\left[\left(k_{e f f}\right)_{n}+\left(\Lambda_{d}\right)_{n}\right], \\
G_{1}=\left\{C_{\psi m}-\frac{(1-\beta)}{\beta} a_{5}^{o}\right\} \psi_{n}^{o}+\left\{C_{T m}-\frac{(1-\beta)}{\beta}\left[a_{5}^{o}+a_{6}^{o}\right]\right\} T_{n}^{o}+\frac{\beta \Delta t}{\Delta z}\left\{K_{n}-K_{n-1}+\left(\frac{\Lambda_{c} K}{D_{T m}}\right)\right\} \\
\quad+\frac{(1-\beta)}{\beta}\left\{a_{5}^{o} \psi_{n-1}^{o}+a_{4}^{o} T_{n-1}^{o}+a_{6}^{o} T_{B}^{o}+\frac{\beta \Delta t}{\Delta z}\left(K_{n}^{o}-K_{n-1}^{o}+\left(\frac{\Lambda_{c} K}{D_{T m}}\right)_{n}^{o}\right)\right\} \\
G_{2}=\left\{C_{\psi T}-\frac{(1-\beta)}{\beta} b_{5}^{o}\right\} \psi_{n}^{o}+\left\{C_{T T}-\frac{(1-\beta) \Delta t}{(\Delta z)^{2}}\left[b_{4}^{o}+b_{6}^{o}\right]\right\} T_{n}^{o}+\frac{(1-\beta)}{\beta}\left\{b_{5}^{o} \psi_{n-1}^{o}+b_{4}^{o} T_{n-1}^{o}+b_{6}^{o} T_{B}^{o}\right\}, \\
\Lambda_{c}=\left(\frac{-D_{T m} \cdot D_{\psi m}}{K+D_{\psi v}}\right)_{n}, \quad \Lambda_{d}=\left(\frac{-D_{T m} \cdot D_{\psi T}}{K+D_{\psi v}}\right)_{n}, \quad K_{n} \text { means } K \text { at node } n, \text { and }
\end{gathered}
$$

Assembling Eqs. 5.3, 5.7, and 5.9, the discretized governing equation (i.e., Eq. 2.5) can be expressed as:

$$
\left[\begin{array}{ccccccc}
M_{1,1} & -M_{1,2} & 0 & 0 & \ldots & 0 & 0 \\
-M_{2,1} & M_{2,2} & -M_{2,3} & 0 & 0 & \ldots & 0 \\
0 & -M_{3,2} & M_{3,3} & -M_{3,4} & 0 & \ldots & 0 \\
0 & 0 & -M_{4,3} & M_{4,4} & -M_{4,5} & \ldots & 0 \\
\ldots & \ldots & \ldots & \ldots & \ldots & \ldots & \ldots \\
0 & 0 & 0 & \ldots & -M_{n-1, n-2} & M_{n-1, n-1} & -M_{n-1, n} \\
0 & 0 & 0 & \ldots & 0 & -M_{n, n-1} & M_{n, n}
\end{array}\right]\left[\begin{array}{c}
\Xi_{1} \\
\Xi_{2} \\
\Xi_{3} \\
\Xi_{4} \\
\ldots \\
\Xi_{n-1} \\
\Xi_{n}
\end{array}\right]=\left[\begin{array}{c}
G G_{1} \\
G G_{2} \\
G G_{3} \\
G G_{4} \\
\ldots \\
G G_{n-1} \\
G G_{n}
\end{array}\right]
$$


where $n$ is the total number of CVs (as shown in Fig. 5.1); $\quad \Xi_{x}=\left[\begin{array}{ll}\psi_{x} & T_{x}\end{array}\right]^{T}$;

In row 1: $G G_{1}=\left[\begin{array}{c}a_{5} \psi_{A}+a_{6} T_{A}+G_{1} \\ b_{5} \psi_{A}+b_{6} T_{A}+G_{2}\end{array}\right], M_{1,1}=\left[\begin{array}{cc}a_{1} & a_{2} \\ b_{1} & b_{2}\end{array}\right]$, and $M_{1,2}=\left[\begin{array}{cc}a_{3} & a_{4} \\ b_{3} & b_{4}\end{array}\right]$ from Eq. 5.7;

From rows 2 to $n-1: G G_{x}=\left[\begin{array}{c}\left(G_{1}\right)_{x} \\ \left(G_{2}\right)_{x}\end{array}\right], M_{x, x+1}=\left[\begin{array}{cc}\left(a_{3}\right)_{x} & \left(a_{4}\right)_{x} \\ \left(b_{3}\right)_{x} & \left(b_{4}\right)_{x}\end{array}\right], M_{x, x}=\left[\begin{array}{cc}\left(a_{1}\right)_{x} & \left(a_{2}\right)_{x} \\ \left(b_{1}\right)_{x} & \left(b_{2}\right)_{x}\end{array}\right]$, and

$M_{x, x-1}=\left[\begin{array}{ll}\left(a_{5}\right)_{x} & \left(a_{6}\right)_{x} \\ \left(b_{5}\right)_{x} & \left(b_{6}\right)_{x}\end{array}\right]$ from Eq. 5.3; $\quad$ subscript $x$ means the row number; $\quad$ and

In row $n: G G_{n, n}=\left[\begin{array}{c}a_{3} \psi_{B}+a_{4} T_{B}+G_{1} \\ b_{3} \psi_{B}+b_{4} T_{B}+G_{2}\end{array}\right]$ and $M_{n, n}=\left[\begin{array}{ll}a_{1} & a_{2} \\ b_{1} & b_{2}\end{array}\right]$ from Eq. 5.9

\section{Initial conditions:}

1. The temperatures at all nodes are at room temperature $\left(\sim 23^{\circ} \mathrm{C}\right)$

2. The relative humidity $(\varphi)$ of dry soil is the same as that of the lab room $(\sim 23 \%)$

3. The initial moisture content in wet soil can be used to calculate the initial matric potential using van Genuchten's method [51] (more details in Appendix A.2).

4. The initial moisture content of a node is the initial moisture content in the soil. Because soil sample is compacted layer by layer to avoid damaging the heat pulse probes, the initial moisture content in the soil may vary.

\subsection{2 - Finite Volume Method-Axisymmetric Formulation}

Since the soil-column geometry in the experiments is axisymmetric, the azimuthal gradient (around the $\theta$-direction in cylindrical coordinate) is assumed to be zero. The following CVs in axisymmetric geometry, with $\Delta r$ and $\Delta x$ being constant, are considered as shown in Fig. 5.2: 

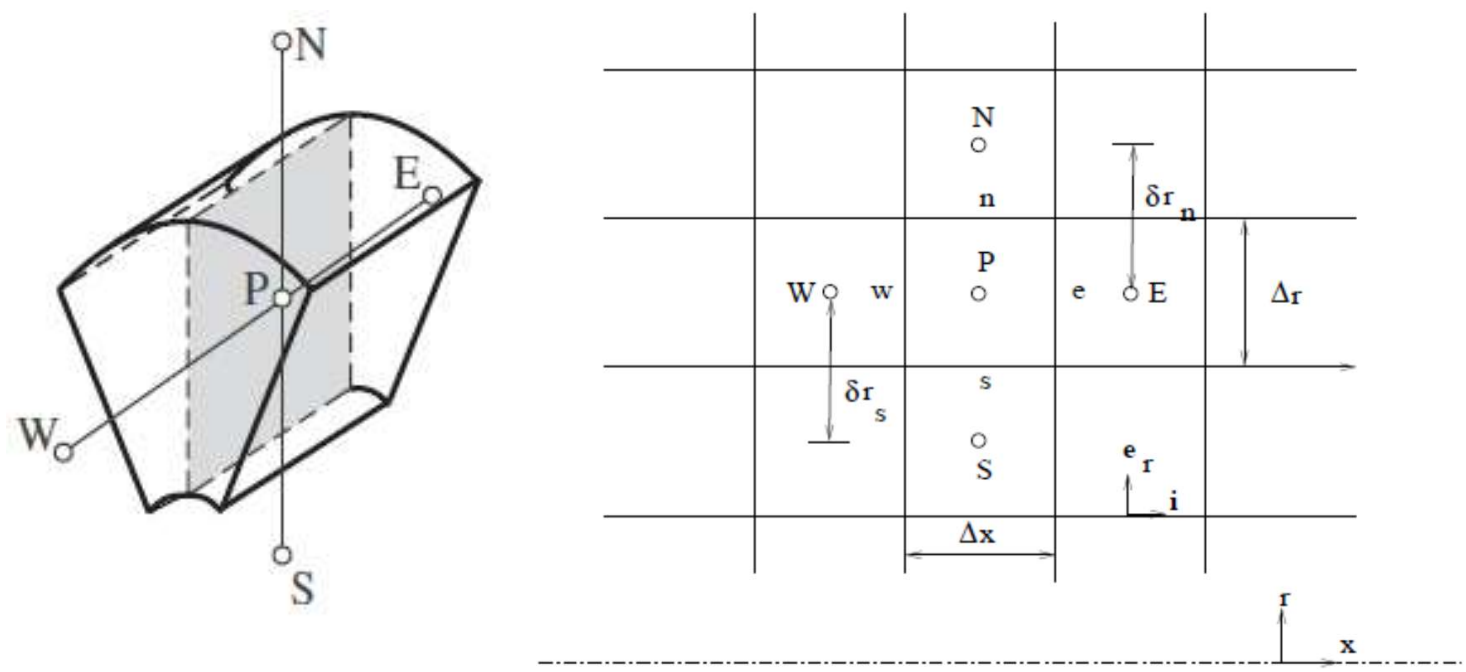

Figure 5.2. Control volume in axisymmetric geometry

Fig. 5.2 is analogous to the $x-y$ geometry in Cartesian coordinate. Before going to the governing equations (Eq. 2.5), a simple diffusion problem in axisymmetric geometry is considered first. Within a $\mathrm{CV}, \Delta \forall=2 \pi r_{P} \Delta r \Delta x$ and the followings are assumed:

1. $\hbar$ represents $\psi$ and $T$ and varies linearly between grid points

2. $\vec{J}=-D \vec{\nabla} \hbar$ is the diffusive flux where $\vec{\nabla}=\frac{\partial}{\partial r} \vec{e}_{r}+\frac{\partial}{\partial x} \vec{i}$ and $D$ is the diffusion coefficient

3. $\vec{\Xi}$ is the face area vectors: $\quad \vec{\Xi}_{e}=2 \pi r_{e} \Delta r \vec{i}, \quad \vec{\Xi}_{w}=-2 \pi r_{w} \Delta r \vec{i}, \quad \vec{\Xi}_{n}=2 \pi r_{n} \Delta x \vec{e}_{r}$, and $\vec{\Xi}_{s}=-2 \pi r_{s} \Delta x \vec{e}_{r}$

where $r$ is the radial distance the face of the $\mathrm{CV}$ is away from the axis. For instance, if $\Delta r$ is constant, $r_{e}=r_{E}=r_{P}$ where $r_{E}$ is the radial distance of the centroid $\mathrm{E}$ (or P) away from the axis. $r_{n}=r_{N}-\Delta r / 2=r_{P}+\Delta r / 2$ and $r_{s}=r_{S}+\Delta r / 2=r_{P}-\Delta r / 2$.

The net diffusion of the $\mathrm{CV}$ is made by integrating the diffusion over the $\mathrm{CV} \mathrm{P}$ :

$$
\int_{\Delta \forall} \vec{\nabla} \cdot \vec{J} d \forall=\int_{A} \vec{J} \cdot d \vec{A}=\sum_{f=n, s, e, w} \vec{J}_{f} \cdot \vec{A}_{f}
$$

As a result, the diffusion fluxes on the faces of a CV in the discretized form are [61]: 


$$
\begin{array}{ll}
\vec{J}_{e} \cdot \vec{\Xi}_{e}=-2 \pi D_{e} r_{e} \Delta r \frac{\hbar_{E}-\hbar_{P}}{\Delta x} & \vec{J}_{w} \cdot \vec{\Xi}_{w}=2 \pi D_{w} r_{w} \Delta r \frac{\hbar_{P}-\hbar_{W}}{\Delta x} \\
\vec{J}_{n} \cdot \vec{\Xi}_{n}=-2 \pi D_{n} r_{n} \Delta x \frac{\hbar_{N}-\hbar_{P}}{\Delta r} & \vec{J}_{s} \cdot \vec{\Xi}_{s}=2 \pi D_{s} r_{s} \Delta x \frac{\hbar_{P}-\hbar_{S}}{\Delta r}
\end{array}
$$

If the diffusive flux only acts in the $x$-direction, the discretized diffusion of Eq. 5.11 is:

$$
\vec{J}_{e} \cdot \vec{\Xi}_{e}=-2 \pi D_{e} \frac{\hbar_{E}-\hbar_{P}}{\Delta x} \quad \vec{J}_{w} \cdot \vec{\Xi}_{w}=2 \pi D_{w} \frac{\hbar_{P}-\hbar_{W}}{\Delta x}
$$

If the diffusive flux only acts in the $y$-direction, the discretized diffusion of Eq. 5.11 is:

$$
\vec{J}_{n} \cdot \vec{\Xi}_{n}=-2 \pi D_{n} \frac{\hbar_{N}-\hbar_{P}}{\Delta y} \quad \vec{J}_{s} \cdot \vec{\Xi}_{s}=2 \pi D_{s} \frac{\hbar_{P}-\hbar_{S}}{\Delta y}
$$

Comparing Figs. 5.1 and 5.2, it can be seen that the $z$-direction in Fig. 5.1 is analogous to the $x$-direction in Fig. 5.2. By looking at Eq. 5.12, it can be seen that the diffusion coefficients in Eq. $5.2\left(D_{\psi m}, D_{T m}, D_{\psi T}\right.$, and $\left.k_{e f f}\right)$ are missing the $2 \pi r \Delta x$ and $2 \pi r \Delta r$ factors when the geometry goes from 1D to axisymmetry. Applying Fig. 5.2 with analogy to the $x-y$ geometry, constant grid size for all CVs, and Eqs. 5.2 and 5.12, the discretized governing equations for the nodes whose CV surfaces do not touch the boundaries of the simulation domain are given by:

$$
\begin{aligned}
& a_{1} \psi_{P}+a_{2} T_{P}=a_{3} \psi_{N}+a_{4} T_{N}+a_{5} \psi_{S}+a_{6} T_{S}+a_{7} \psi_{E}+a_{8} T_{E}+a_{9} \psi_{W}+a_{10} T_{W}+G_{1} \\
& b_{1} \psi_{P}+b_{2} T_{P}=b_{3} \psi_{N}+b_{4} T_{N}+b_{5} \psi_{S}+b_{6} T_{S}+b_{7} \psi_{E}+b_{8} T_{E}+b_{9} \psi_{W}+b_{10} T_{W}+G_{2}
\end{aligned}
$$

where $a_{1}=C_{\psi m}+a_{3}+a_{5}+a_{7}+a_{9}, \quad a_{2}=C_{T m}+a_{4}+a_{6}+a_{8}+a_{10}$,

$$
\begin{gathered}
b_{1}=C_{\psi T}+b_{3}+b_{5}+b_{7}+b_{9}, \quad b_{2}=C_{T T}+b_{4}+b_{6}+b_{8}+b_{10}, \\
a_{3}=\frac{\beta \Delta t}{(\Delta r)^{2}} \frac{r_{n}}{r_{P}}\left(\frac{D_{\psi m}}{2 \pi}\right)_{n}, a_{4}=\frac{\beta \Delta t}{(\Delta r)^{2}} \frac{r_{n}}{r_{P}}\left(\frac{D_{T m}}{2 \pi}\right)_{n}, a_{5}=\frac{\beta \Delta t}{(\Delta r)^{2}} \frac{r_{s}}{r_{P}}\left(\frac{D_{\psi m}}{2 \pi}\right)_{s}, a_{6}=\frac{\beta \Delta t}{(\Delta r)^{2}} \frac{r_{s}}{r_{P}}\left(\frac{D_{T m}}{2 \pi}\right)_{s}, \\
a_{7}=\frac{\beta \Delta t}{(\Delta x)^{2}} \frac{r_{e}}{r_{P}}\left(\frac{D_{\psi m}}{2 \pi}\right)_{e}, a_{8}=\frac{\beta \Delta t}{(\Delta x)^{2}} \frac{r_{e}}{r_{P}}\left(\frac{D_{T m}}{2 \pi}\right)_{e}, a_{9}=\frac{\beta \Delta t}{(\Delta x)^{2}} \frac{r_{w}}{r_{P}}\left(\frac{D_{\psi m}}{2 \pi}\right)_{w}, a_{10}=\frac{\beta \Delta t}{(\Delta x)^{2}} \frac{r_{w}}{r_{P}}\left(\frac{D_{T m}}{2 \pi}\right)_{w},
\end{gathered}
$$




$$
\begin{aligned}
b_{3}=\frac{\beta \Delta t}{(\Delta r)^{2}} \frac{r_{n}}{r_{P}}\left(\frac{D_{\psi T}}{2 \pi}\right)_{n}, b_{4}=\frac{\beta \Delta t}{(\Delta r)^{2}} \frac{r_{n}}{r_{P}}\left(\frac{k_{\text {eff }}}{2 \pi}\right)_{n}, b_{5}=\frac{\beta \Delta t}{(\Delta r)^{2}} \frac{r_{s}}{r_{P}}\left(\frac{D_{\psi T}}{2 \pi}\right)_{s}, b_{6}=\frac{\beta \Delta t}{(\Delta r)^{2}} \frac{r_{s}}{r_{P}}\left(\frac{k_{\text {eff }}}{2 \pi}\right)_{s}, \\
b_{7}=\frac{\beta \Delta t}{(\Delta x)^{2}} \frac{r_{e}}{r_{P}}\left(\frac{D_{\psi T}}{2 \pi}\right)_{e}, b_{8}=\frac{\beta \Delta t}{(\Delta x)^{2}} \frac{r_{e}}{r_{P}}\left(\frac{k_{e f f}}{2 \pi}\right)_{e}, b_{5}=\frac{\beta \Delta t}{(\Delta x)^{2}} \frac{r_{w}}{r_{P}}\left(\frac{D_{\psi T}}{2 \pi}\right)_{w}, b_{10}=\frac{\beta \Delta t}{(\Delta x)^{2}} \frac{r_{w}}{r_{P}}\left(\frac{k_{e f f}}{2 \pi}\right)_{w}, \\
G_{1}=\left\{C_{\psi m}-\frac{(1-\beta)}{\beta}\left[a_{3}^{o}+a_{5}^{o}+a_{7}^{o}+a_{9}^{o}\right]\right\} \psi_{P}^{o}+\left\{C_{T m}-\frac{(1-\beta)}{\beta}\left[a_{4}^{o}+a_{6}^{o}+a_{8}^{o}+a_{10}^{o}\right]\right\} T_{P}^{o}, \\
+\frac{(1-\beta)}{\beta}\left\{a_{3}^{o} \psi_{N}^{o}+a_{5}^{o} \psi_{S}^{o}+a_{4}^{o} T_{N}^{o}+{ }_{6}^{o} T_{S}^{o}+\right\}+K_{\text {term }} \\
G_{2}=\left\{C_{\psi T}-\frac{(1-\beta)}{\beta}\left[b_{3}^{o}+b_{5}^{o}+b_{7}^{o}+b_{9}^{o}\right]\right\} \psi_{P}^{o}+\left\{C_{T T}-\frac{(1-\beta)}{\beta}\left[b_{4}^{o}+b_{6}^{o}+b_{8}^{o}+b_{10}^{o}\right]\right\} T_{P}^{o} . \\
+\frac{(1-\beta)}{\beta}\left\{b_{3}^{o} \psi_{N}^{o}+b_{5}^{o} \psi_{S}^{o}+b_{4}^{o} T_{N}^{o}+b_{6}^{o} T_{S}^{o}\right\}
\end{aligned}
$$

If the gravitational vector $\vec{g}$ is in the $r$-direction:

$$
K_{\text {term }}=\frac{\beta \Delta t}{(\Delta r)^{2}}\left[K_{n} \frac{r_{n}}{2 \pi r_{P}}-K_{s} \frac{r_{s}}{2 \pi r_{P}}\right]+\frac{(1-\beta) \Delta t}{(\Delta r)^{2}}\left[K_{n}^{o} \frac{r_{n}}{2 \pi r_{P}}-K_{s}^{o} \frac{r_{s}}{2 \pi r_{P}}\right]
$$

If the gravitational vector $\vec{g}$ is in the $x$-direction:

$$
K_{\text {term }}=\frac{\beta \Delta t}{(\Delta x)^{2}}\left[K_{e} \frac{r_{e}}{2 \pi r_{P}}-K_{w} \frac{r_{w}}{2 \pi r_{P}}\right]+\frac{(1-\beta) \Delta t}{(\Delta x)^{2}}\left[K_{e}^{o} \frac{r_{e}}{2 \pi r_{P}}-K_{w}^{o} \frac{r_{w}}{2 \pi r_{P}}\right]
$$

The initial conditions are similar to the one-dimension case's. The boundary conditions for the axisymmetric geometry (whose coordinates are shown in Fig. 5.2) are:

1. Along the $x$-direction: same as those in the one-dimension case

2. Along the $r$-direction:

a. at $r=0$, the heat and moisture fluxes at any point on the cylindrical axis are zero, i.e.,

$$
\left.\frac{\partial T}{\partial r}\right|_{r=0}=\left.\frac{\partial \psi}{\partial r}\right|_{r=0}=0
$$


b. at $r=r_{i n}, T\left(r_{i n}, x\right)=$ known, where $r_{i n}$ is the inner radius of the stainless-steel tube for the soil column. A simplified version of the temperature $T\left(r_{i n}, x\right)$ is adopted by assuming a linear temperature distribution along the soil column wall in the $x$-direction from the top plate to the bottom plate, due to much higher thermal conductivity of stainless steel than the ones of soils and insulation (as shown in Fig. 6.9).

c. at $r=r_{i n}$, the stainless-steel tube wall is an impermeable surface for moisture. Therefore, as a result of Eq. 5.4, matric potential gradients at $r=r_{\text {in }}$ for a vertical soil column are as follows:

$$
\left.\frac{\partial \psi}{\partial r}\right|_{r=r_{\text {in }}}=\frac{-1}{K+D_{\psi v}}\left(\left.D_{T m} \frac{\partial T}{\partial r}\right|_{r=r_{\text {in }}}\right)
$$

Under certain nodal numbering ways for 2D as well as axisymmetric geometries, the solution time is shorter. However, since the $2 \mathrm{D}$ nodal numbering method varies with each reader, the assembled discretized governing equations (similar to Eq. 5.10) for axisymmetric geometry is left for the reader to derive.

\section{3 - Development of Computer Codes}

Since there are numerous equations for the material properties and the solving mechanism of the governing equations, it is best to create functions (or objects) for handling certain tasks so that the main program can call out the functions quickly and the programming process can be more organized and streamlined. There are many programming languages currently in use. Matlab was chosen as it is one of the popular and high level programming languages in the world. In addition, Matlab has many built-in functions which are readily to be called. More about Matlab programming for this thesis is shown in Appendix B.

\subsubsection{Solution Method for One-Dimensional Heat and Mass Transfer}

Generally speaking, the matrix equation in the finite volume method after assembling all nodes (e.g., Eq. 5.10) can be solved using Gaussian elimination or tridiagonal matrix algorithm (TDMA). The TDMA is faster than the Gaussian elimination but the discretized governing 
equation (Eq. 5.10) does not form a tridiagonal matrix in all cases. In isothermal cases, the temperature does not change with time, so the discretized governing equations produce a tridiagonal matrix after all discretized nodes are assembled and the TDMA can be applied to calculate the matric potential.

Another method to solve the discretized Eq. 2.5 is to apply decoupling of $\psi$ and $T$. Within a time step, nodal $T$ values are guessed and used to solve for the nodal $\psi$ values using Eq. 2.5a. The calculated nodal $\psi$ values are then used to calculate the nodal $T$ values using Eq. $2.5 \mathrm{~b}$. The new nodal $T$ values are used to re-calculate nodal $\psi$ values which are then used to calculate the nodal $T$ values. The procedure is repeated until convergence. As indicated by Karki and Pantakar [62], this decoupling method can become slowly convergent if the coupling is strong.

A faster converging method to solve Eq. 5.3 is the partial elimination algorithm (PEA) [63]. Eqs. 5.3, 5.7, and 5.9, can be expressed as follows:

$$
\begin{aligned}
& a_{1} \psi_{P}+a_{2} T_{P}=\sum a_{F}^{\psi} \psi_{F}+G_{3} \\
& b_{1} \psi_{P}+b_{2} T_{P}=\sum a_{F}^{T} T_{F}+G_{4}
\end{aligned}
$$

where $\sum a_{F}^{\psi} \psi_{F}=a_{3} \psi_{N}+a_{5} \psi_{S}, G_{3}=G_{1}+a_{4} T_{N}+a_{6} T_{S}$

$$
\sum a_{F}^{T} T_{F}=b_{3} T_{N}+b_{5} T_{S}, \quad G_{4}=G_{2}+b_{4} \psi_{N}+b_{6} \psi_{S}
$$

The expressions for $a_{\mathrm{i}}$ and $b_{\mathrm{i}}, G_{1}$ and $G_{2}$ can be derived from Eqs. 5.3, 5.7, and 5.9 for the nodes

Multiplying Eq. 5.16a with $b_{2}$ and Eq. 5.16b with $a_{2}$ and isolating $\psi_{P}$ :

$$
a_{P}^{\psi} \psi_{P}=\sum a_{F}^{\psi} \psi_{F}+B_{P}^{\psi}
$$

where $a_{P}^{\psi}=\frac{a_{1} b_{2}-a_{2} b_{1}}{b_{2}}$ and $B_{P}^{\psi}=G_{3}-\frac{a_{2}}{b_{2}}\left(\sum a_{F}^{T} T_{F}+G_{4}\right)$

Similarly, multiplying Eq. 5.16a with $b_{1}$ and Eq. 5.16b with $a_{1}$ and isolating $T_{P}$ : 


$$
a_{P}^{T} T_{P}=\sum b_{F}^{T} T_{F}+B_{P}^{T}
$$

where $a_{P}^{T}=\frac{a_{1} b_{2}-a_{2} b_{1}}{a_{1}}$ and $B_{P}^{T}=G_{4}-\frac{b_{1}}{a_{1}}\left(\sum a_{F}^{\psi} \psi_{F}+G_{3}\right)$

Since the convection and diffusion coefficients are highly non-linear, an iterative approach is adopted from Moukalled and Saleh [63]:

1. Assume reasonable values for $\psi\left(\psi_{\text {guess }}\right)$ and $T\left(T_{\text {guess }}\right)$

2. Calculate the physical properties of soil using $\psi_{\text {guess }}$ and $T_{\text {guess }}$

3. Calculate the coefficients in Eqs. 5.3a, 5.7a, and 5.9a using $\psi_{\text {guess }}$ and $T_{\text {guess }}$

4. Calculate the coefficients in Eq. 5.17 using $\psi_{\text {guess }}$ and $T_{\text {guess }}$

5. Using TDMA, solve for nodal $\psi$ values $\left(\psi^{i}\right)$ where $i$ means current iteration and

$$
\left.\frac{a_{P}^{\psi}}{\varpi}\right|_{\substack{\psi_{\text {guess }} \\ \psi_{\text {guess }}}} \psi_{P}^{i}=\left.\sum a_{F}^{\psi}\right|_{T_{T_{\text {guess }}}} \psi_{F}^{i}+\left.B_{P}^{\psi}\right|_{T_{\text {guess }}}+\left.\frac{1-\varpi}{\varpi} a_{P}^{\psi}\right|_{\substack{T_{\text {guness }} \\ \psi_{\text {guness }}}} \psi_{P}^{i-1}
$$

6. Calculate the coefficients in Eqs. 5.3b, 5.7b, and 5.9b using $\psi^{i}$ and $T_{\text {guess }}$

7. Calculate the coefficients in Eq. 5.18 using $\psi^{i}$ and $T_{\text {guess }}$

8. Using TDMA, solve for nodal $T$ values $\left(T^{i}\right)$ where $i$ means current iteration and

$$
\left.\frac{a_{P}^{T}}{\varpi}\right|_{\substack{\psi^{i} \\ T_{\text {guess }}}} T_{P}^{i}=\left.\sum a_{F}^{T}\right|_{T_{\text {guess }}{ }^{i}} T_{F}^{i}+\left.B_{P}^{T}\right|_{T_{\text {guess }}^{i}}+\left.\frac{1-\varpi}{\varpi} a_{P}^{T}\right|_{T_{\text {guess }}{ }^{i}} T_{P}^{i-1}
$$

9. Compare $\psi^{i}$ and $T^{i}$ with the corresponding $\psi_{\text {guess }}$ and $T_{\text {guess }}$ as:

$$
\varepsilon_{\psi}=\frac{\psi^{i}-\psi_{\text {guess }}}{\psi_{\text {guess }}} \quad \text { and } \quad \varepsilon_{T}=\frac{T^{i}-T_{\text {guess }}}{T_{\text {guess }}}
$$

10. If $\varepsilon_{\psi}<0.001$ and $\varepsilon_{T}<0.001$, proceed to the next time step. Otherwise, repeat steps $1-9$ with $\psi_{\text {guess }}=\psi^{i}$ and $T_{\text {guess }}=T^{i}$ and $i$ in steps $5-9$ becomes $i+1$ and $i-1$ becomes $i$ 


\subsubsection{Solution Method for Axisymmetric Two-Dimensional Heat and Mass Transfer}

Similar to the one-dimensional situation, the solution method can be achieved by making some modifications to Eq. 5.16 as follow:

1. Apply Eq. 5.15 to Eqs. 5.17 and 5.18

2. Let $\sum a_{F}^{\psi} \psi_{F}=a_{3} \psi_{N}+a_{5} \psi_{S}+a_{7} \psi_{E}+a_{9} \psi_{W}$ and $G_{3}=G_{1}+a_{4} T_{N}+a_{6} T_{S}+a_{8} T_{E}+a_{10} T_{W}$

3. Let $\sum a_{F}^{T} T_{P}=b_{3} T_{N}+b_{5} T_{S}+b_{7} T_{E}+b_{9} T_{W}$ and $G_{4}=G_{2}+b_{4} \psi_{N}+b_{6} \psi_{S}+b_{8} \psi_{E}+b_{10} \psi_{W}$

4. Use Eqs. 5.17 and 5.18 for the partial elimination algorithm

5. Apply the iteration procedures in Section 5.3.1 to solve the discretized governing equations

\section{4 - Summary}

The finite volume method (FVM) is used to discretized the governing partial differential equations of coupled heat and mass transfer. One advantage of using the FVM is its global conservation of heat and mass transfer. One-dimensional and axisymmetric heat and mass transfer finite-volume formulations have been developed. Due to the highly non-linear nature of the coefficients in the governing equations (Eq. 2.5) and numerous material properties, plenty customized functions have been created in Matlab to make the programming more organized and streamlined.

There are many methods to solve the discretized equations (e.g, Eq. 5.3). One method is to apply decoupling of $\psi$ and $T$. Nodal values of $T$ are first guessed and used to solve for nodal $\psi$ values which are again used to calculate the nodal $T$ values. The procedure is repeated until convergence is reached within a time step. After convergence is reached, $\psi$ and $T$ of next time step can be calculated. As indicated by Karki and Pantakar [62], the decoupling method can become slowly convergent as the coupling becomes stronger (i.e., $\psi$ and $T$ are more dependent on each other).

Another method that can reach convergence faster is the partial elimination algorithm (PEA) which makes the coupling between $\psi$ and $T$ weaker [62]. PEA isolates $\psi$ and $T$ to one side of each 
equation in the discretized equations (e.g, Eq. 5.3). In this method, $\psi^{i}$ is first solved for using values of $\psi^{i-1}, \psi_{\text {guess }}$ and $T_{\text {guess }}$, and then $T$ is solved for using $T^{i-1}, T_{\text {guess }}$ and $\psi^{i}$ [63]. This procedure is repeated until convergence is reached within a time step.

The next chapter will explore the heat and moisture simulations in soils. 


\section{CHAPTER 6 - NUMERICAL STUDY OF HEAT AND MOISTURE TRANSFER IN SOILS}

\section{1 - Introduction}

Chapter 5 presented the discretization of the governing equations (Eq. 2.5) using the FVM and some formulations to numerically study the heat and mass transfer in the soil. Ideally, threedimensional formulations of the discretized governing equations is the best; but it is the most challenging to deal with. As a result, due to the design of the cylindrical soil column used in the present experimental study, the governing equations can be simplified into axisymmetric or onedimensional formulations.

It is the easiest to first study the heat and mass transfer in soil using the one-dimensional formulations and experimental data. However, over simplification can be problematic and inaccurate to be used in the real world. Consequently, the following are the purposes of this chapter:

1. A numerical simulation using COMSOL will be performed to assess whether onedimensional heat transfer condition exists in the experimental soil column.

2. Numerical solutions of Eq. 2.5 are verified with analytical solutions to ensure the Matlab codes work properly.

3. Numerical simulations (using Eq. 2.5) of different heating cases, according to the experiments, will also be presented.

\section{2 - Finite Element Simulations using COMSOL}

Ideally, the soil column should be as large and high as possible with the height being at least 10 times more than the diameter. However, due to the tight budget of the research and the limited available soil samples, it has been decided that the soil column would be a stainless steel 304 tube of $15.1 \mathrm{~cm}$ length, $6.35 \mathrm{~cm}$ inner diameter, and $7.62 \mathrm{~cm}$ outer diameter. To simplify the finite element (FE) simulations using COMSOL, the square aluminum plates were modeled as round 
plates with $20.32 \mathrm{~cm}$ diameter so that the entire experimental soil cell can be modelled as an axisymmetric problem. The overall geometry of the model in COMSOL is shown in Fig. 6.1. For more design details about the soil cell, please refer to Hedayati-Dezfooli [27]. The thermal properties of all materials for the FE simulations using COMSOL are tabulated in Table 6.1.

Table 6.1. Thermal properties used in finite element simulations using COMSOL.

\begin{tabular}{|c|c|c|c|c|}
\hline Material & $\rho\left(\mathrm{kg} / \mathrm{m}^{3}\right)$ & $\boldsymbol{k}(\mathbf{W} / \mathbf{m} \cdot \mathbf{K})$ & $C_{p}(\mathrm{~J} / \mathrm{kg} \cdot \mathrm{K})$ & Component \\
\hline $\begin{array}{l}\text { Stainless steel } \\
\text { (AISI 304) }\end{array}$ & 7900 & 14.9 & 477 & $\begin{array}{l}\text { Inner and outer tubes } \\
\text { top and bottom lids } \\
\text { of soil column }\end{array}$ \\
\hline $\begin{array}{l}\text { Aluminum } \\
\text { (Alloy 2024) }\end{array}$ & 2770 & 177 & 875 & $\begin{array}{l}\text { Heating and cooling } \\
\text { plates }\end{array}$ \\
\hline $\begin{array}{l}\text { Matilda soil } \\
(\mathrm{SR}=0.00)\end{array}$ & 1495 & $(*)$ & $(*)$ & Dry soil \\
\hline $\begin{array}{l}\text { Matilda soil } \\
(\mathrm{SR}=0.50)\end{array}$ & 1510 & $(*)$ & $(*)$ & Wet soil \\
\hline Fiberglass & 10 & 0.04 & 700 & $\begin{array}{l}\text { Inner and outer } \\
\text { insulation }\end{array}$ \\
\hline $\begin{array}{l}\text { Thermally } \\
\text { conductive } \\
\text { plastic }\end{array}$ & 1100 & 3.33 & 2010 & Heat flux meter \\
\hline
\end{tabular}

Due to limited computing power, memory and time constraint, the finest mesh of the finite element (FE) model is shown in Fig. 6.2. The mesh's minimum element size is $12.0 \mu \mathrm{m}$ at the heat flux meters (HFMs) while the maximum is $2.8 \mathrm{~mm}$ at the outer insulation. There are 40,900 elements and the simulation time is 78.5 mins. Matilda soil (a loamy sand with $71 \%$ sand, $25.4 \%$ silt, and 3.6\% clay [57]) with saturation ratios (SRs) of 0.00 and 0.50 are used for the simulations. The channels in the aluminum plates are assumed to have constant wall temperatures. (In reality, the channel wall temperatures are due to water circulating through the channels). The channel walls in the top and bottom plates are set at $90^{\circ} \mathrm{C}$ and $10^{\circ} \mathrm{C}$, respectively, as shown in Fig. 6.2 along with the convection boundary conditions at the surfaces of the outer insulation. As an initial condition, the entire soil cell is set at the room temperature of $22^{\circ} \mathrm{C}$. The built-in porous media model in COMSOL software package is used for the soil. 
(m)

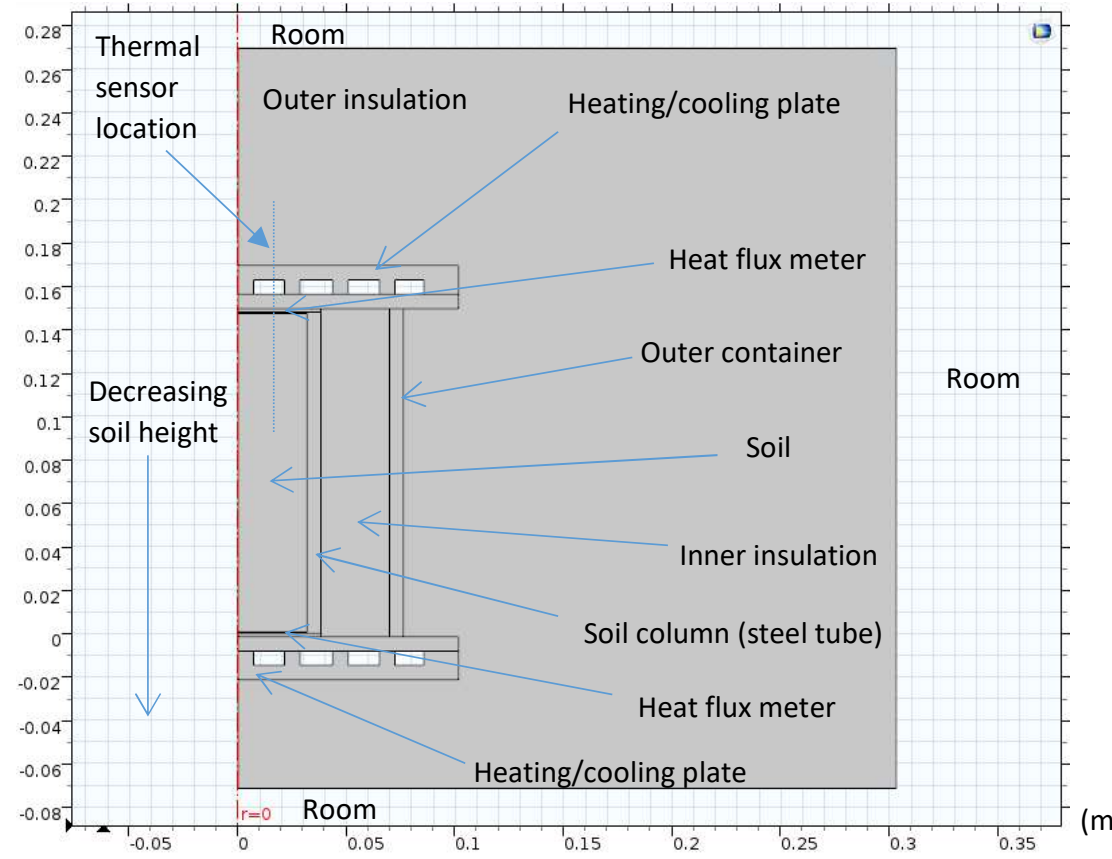

Figure 6.1. Geometry of the soil cell for finite element simulations using COMSOL.

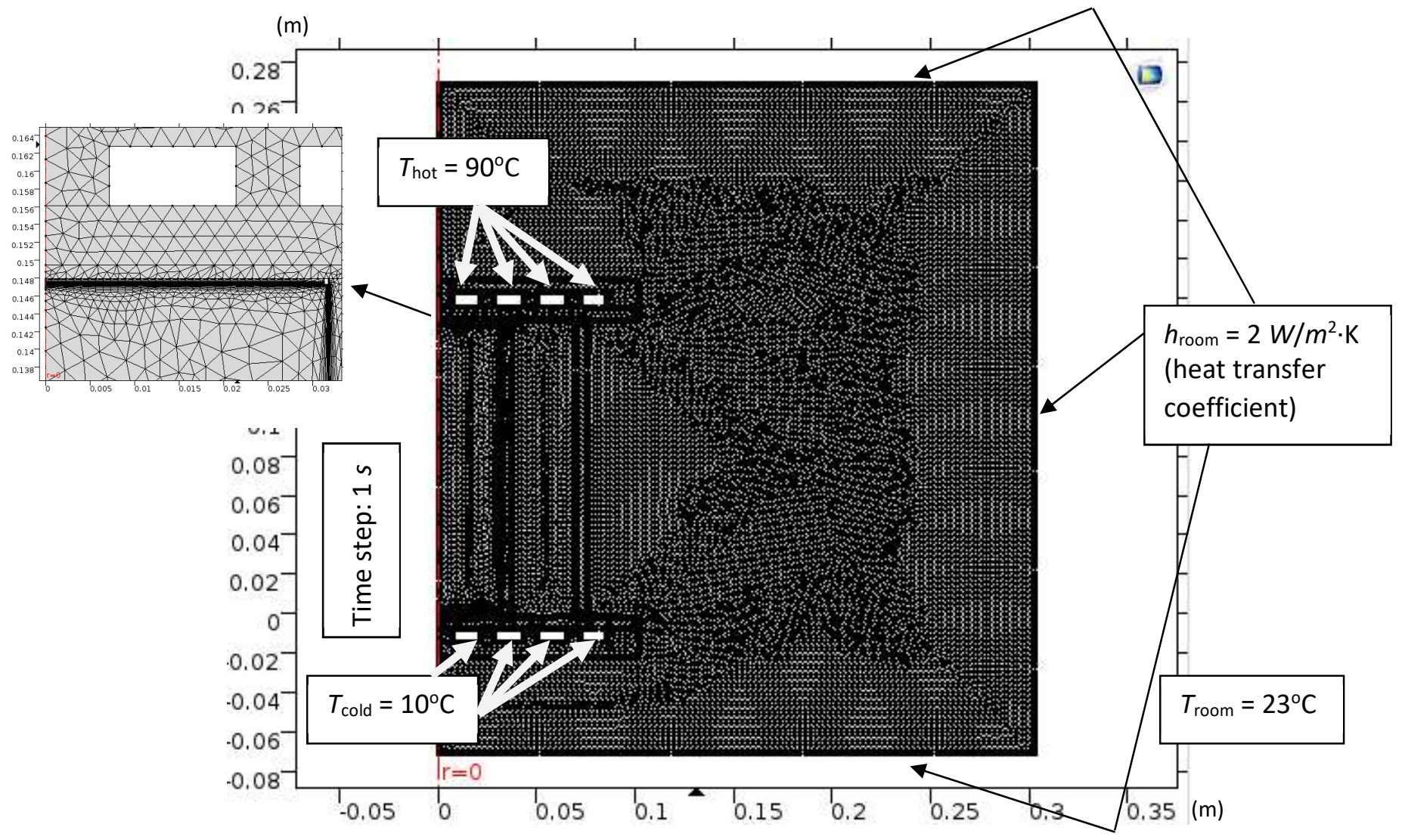

Figure 6.2. Finest mesh of the finite element model using COMSOL. 
Fig. 6.3 shows the changes in simulated axial heat flux at the centerline at different times when the number of elements is varied in the COMSOL model. As shown in the figure, the axial heat flux changes slightly when the number of elements is increased. The results between 31,509 and 40,900 elements only differ by about $2 \%$. As mentioned before, due to limited computing power, memory and time constraint, the attainable finest mesh of 40,900 elements is deemed to be sufficient for the FE simulations using COMSOL. The time increment for showing the results in Figs. $5.3-5.5$ and $5.7-5.8$ is indicated in the figures.
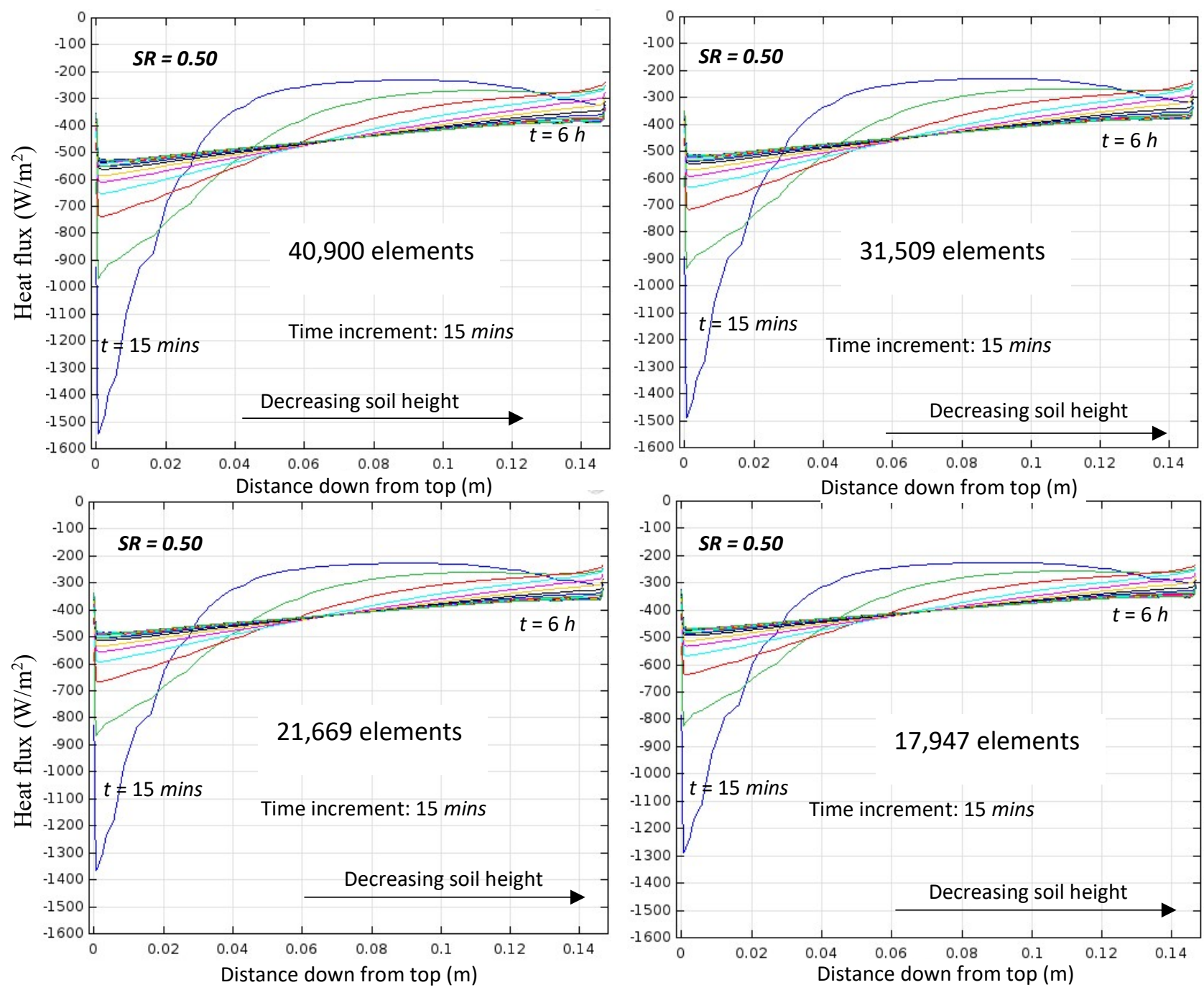

Figure 6.3. Simulated axial heat fluxes along the centerline of the soil column at different times for different number of elements in the FE model using COMSOL. 
From the FE simulations, the transient temperature distributions along the centerline of the soil column are shown in Fig. 6.4 for $\mathrm{SR}=0.00$ and 0.50 . As it can be seen, at the beginning of heating, the soil column is under high transient heat transfer at the top of soil column, due to large temperature gradient, and the temperature distribution along the centerline is curve-shaped and non-linear. However, after a long time, steady state is reached and the temperature distribution along the centerline is almost linear. Moreover, due to higher thermal conductivity of the wet soil $(\mathrm{SR}=0.50)$, more heat is transferred from the heating plate to the cooling plate and the soil column experiences slightly lower temperatures with about the same temperature gradient, comparing to the case of dry soil $(\mathrm{SR}=0.00)$.
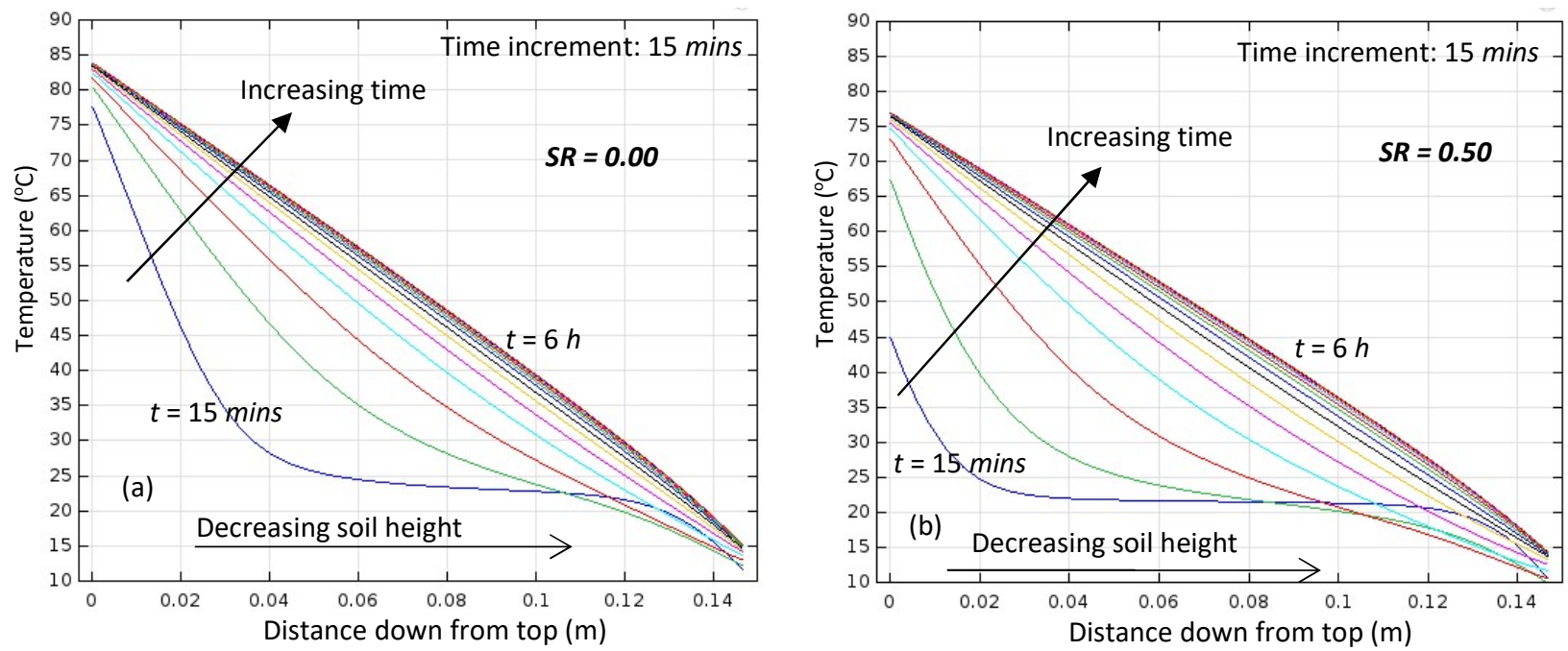

Figure 6.4. Temperature distribution along the centerline of the soil column at different times for

(a) $S R=0.00$ and (b) $S R=0.50$.

Fig. 6.5 shows the axial heat fluxes in the axial along the centerline of the soil column. Similar to Fig. 6.4, the heat flux distribution at the beginning of heating is curve-shaped, while it becomes linear after a long time. The reason is that, at steady state, there is a steady heat loss from the soil column to the ambient air via the insulation. In addition, the radial heat flux is mostly much smaller than the axial one as time increases. Also, from the isotherms and heat flows shown in Fig. 6.6, it can be seen that after 6 hours of heating, the heat flow along the axial direction of the soil is steady. Moreover, the heat flow vectors along the centerline appear to be collinear with the centerline, further indicating the axial heat flow is much more significant than the radial heat flow at any point on the centerline. 

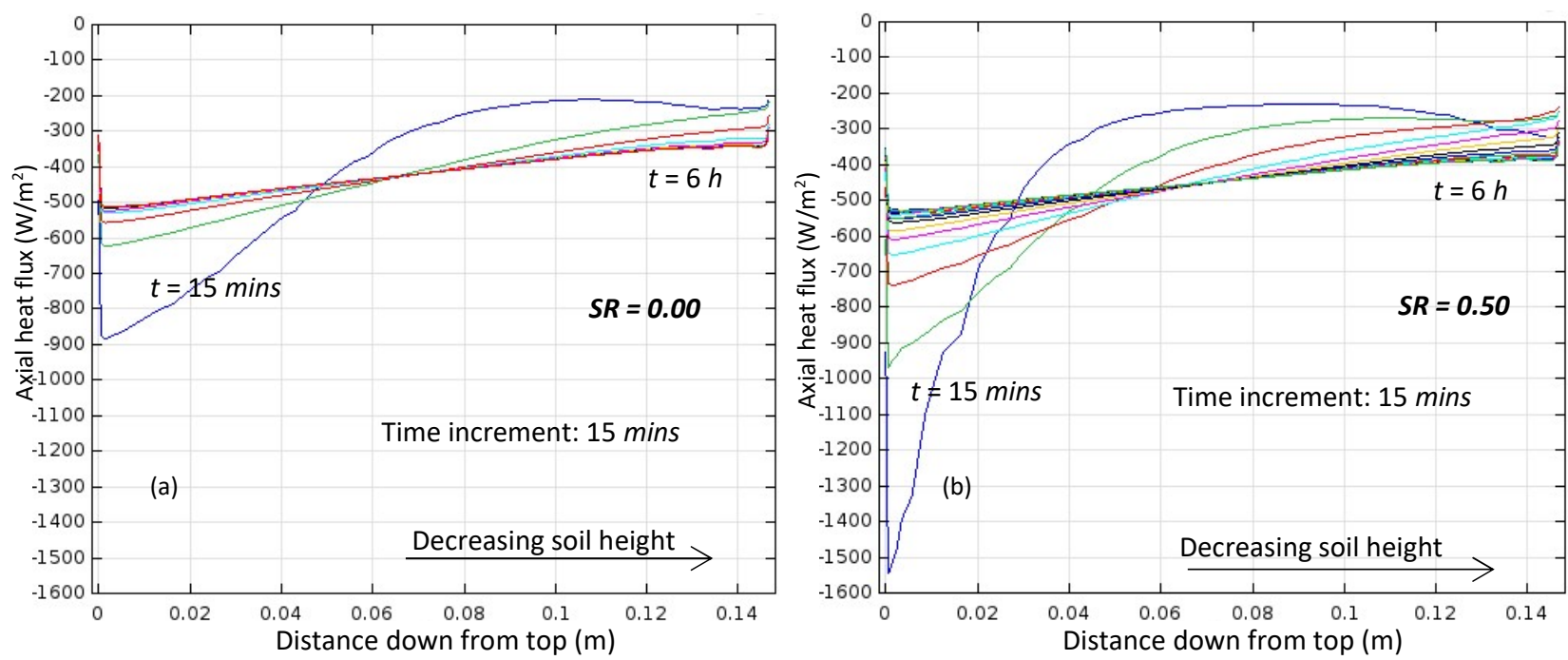

Figure 6.5. Simulated axial heat flux along the centerline of the soil column for (a) $S R=0.00$

$$
\text { and (b) } S R=0.50 \text {. }
$$
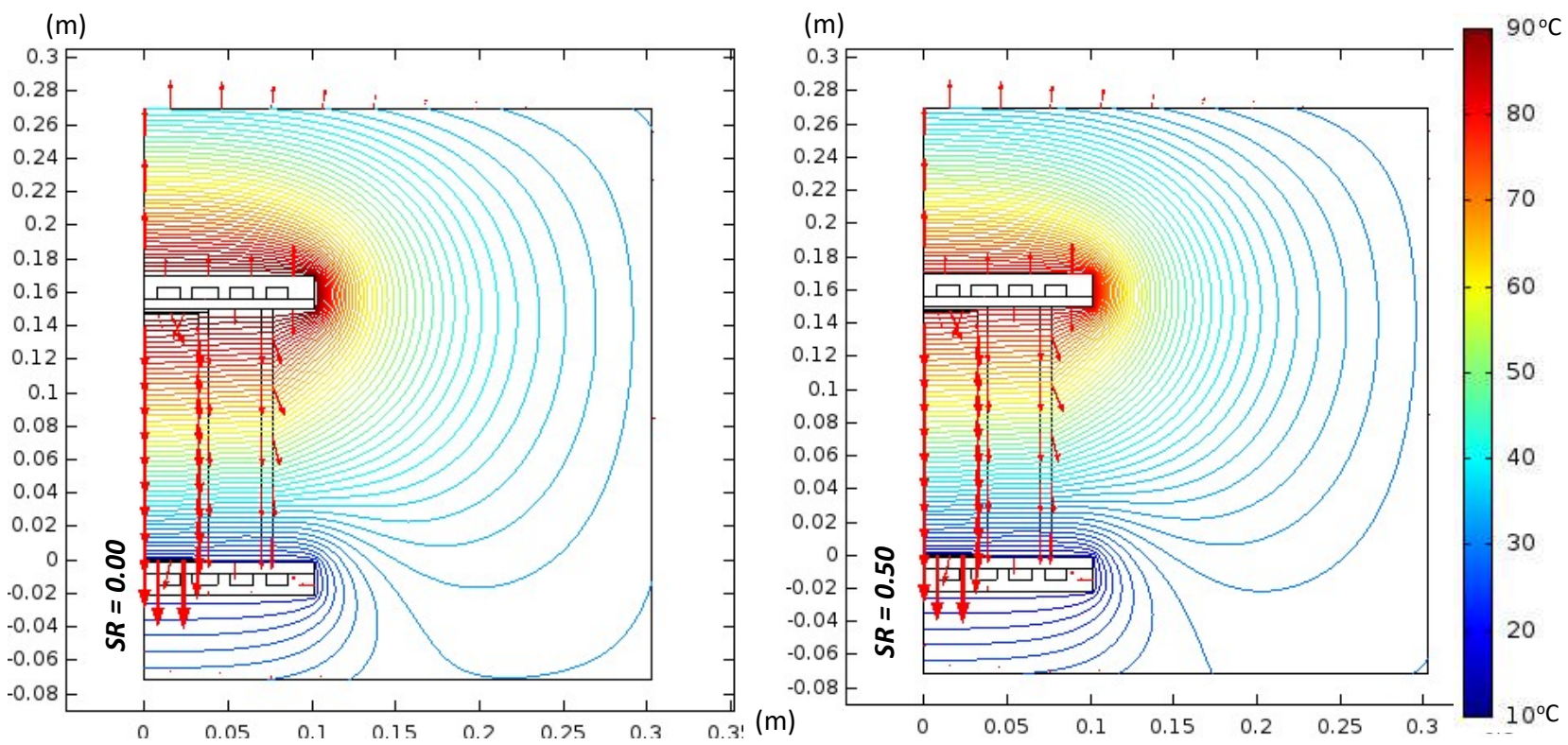

Figure 6.6. Simulated isotherms and heat flow vectors at $t=6$ hours.

Fig. 6.7 shows the axial and radial heat fluxes along the height of the soil column at the temperature sensor location $(r=1.675 \mathrm{~cm})$ in the actual temperature-sensing needles of the heat pulse probe. In the beginning, e.g., at 15 mins, because of high thermal conductivity of stainless steel compared to the thermal conductivity of soil, heat first flows from the hot aluminum plate down the stainless-steel tube wall and then flows to the soil, which is initially at the room temperature, resulting significant radial heat flux as much as about $-200 \mathrm{~W} / \mathrm{m}^{2}$ (that is about $50 \%$ 
of the corresponding axial heat flux at the same location). However, the radial heat flux at the temperature sensor location becomes relatively insignificant compared to the corresponding axial one after 60 mins. At steady state, e.g., at 6 hours, there is only a small amount of radial heat loss $(<5 \%)$ compared to the corresponding axial one. Furthermore, the axial heat flux along the centerline of the soil column (Fig. 6.5) is similar to the corresponding axial heat flux at the temperature sensor location (Fig. 6.7). As a result, one-dimensional heat flow conditions (in the axial direction) can be assumed for the soil column, after 60 mins.
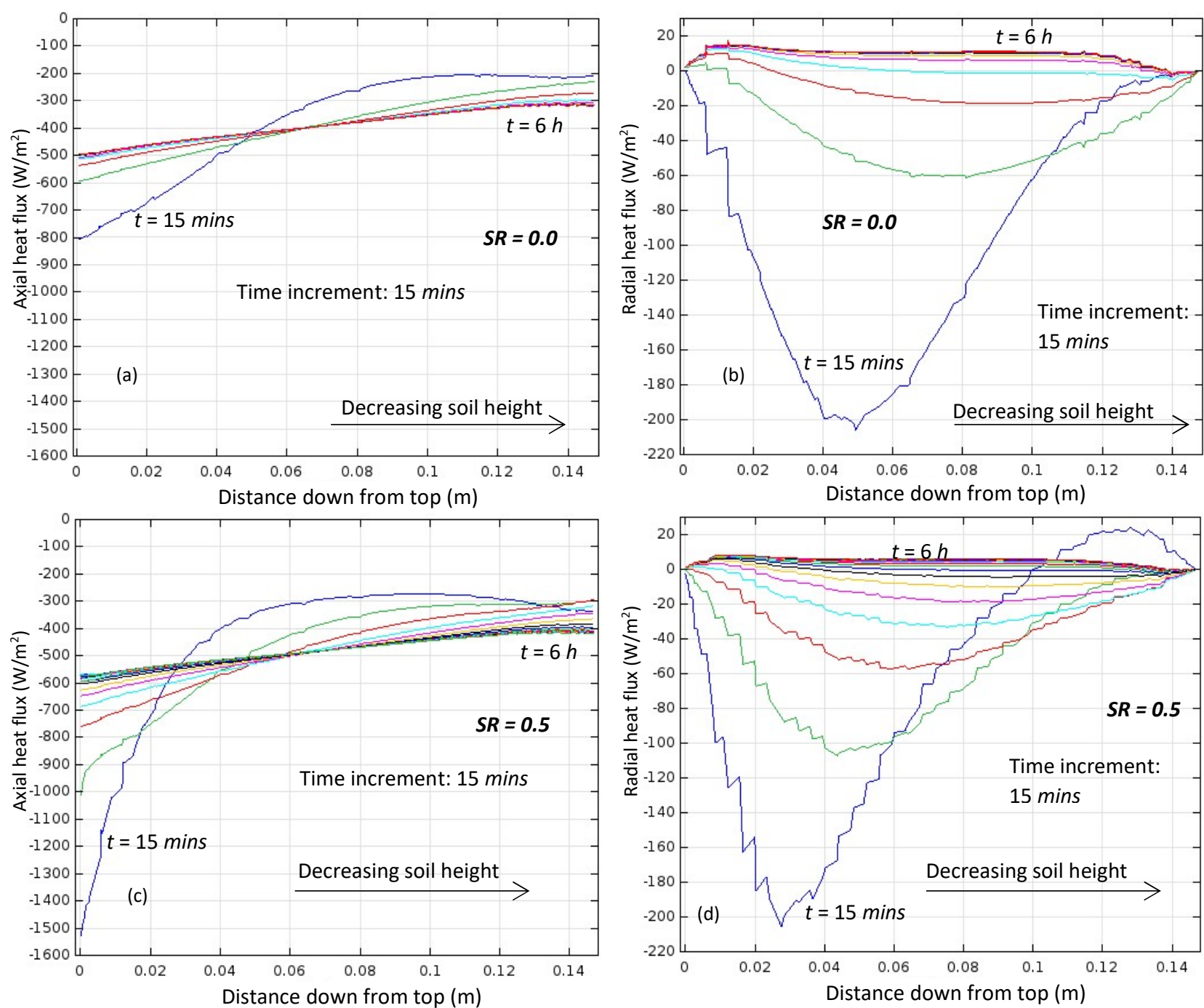

Figure 6.7. Simulated heat fluxes along the height of the soil column at the temperature sensor location $(r=1.675 \mathrm{~cm})$ for $($ a) axial heat flux $(S R=0)$, (b) radial heat flux $(S R=0)$, (c) axial heat flux $(S R=0.5)$, and $(d)$ radial heat flux $(S R=0.5)$. 

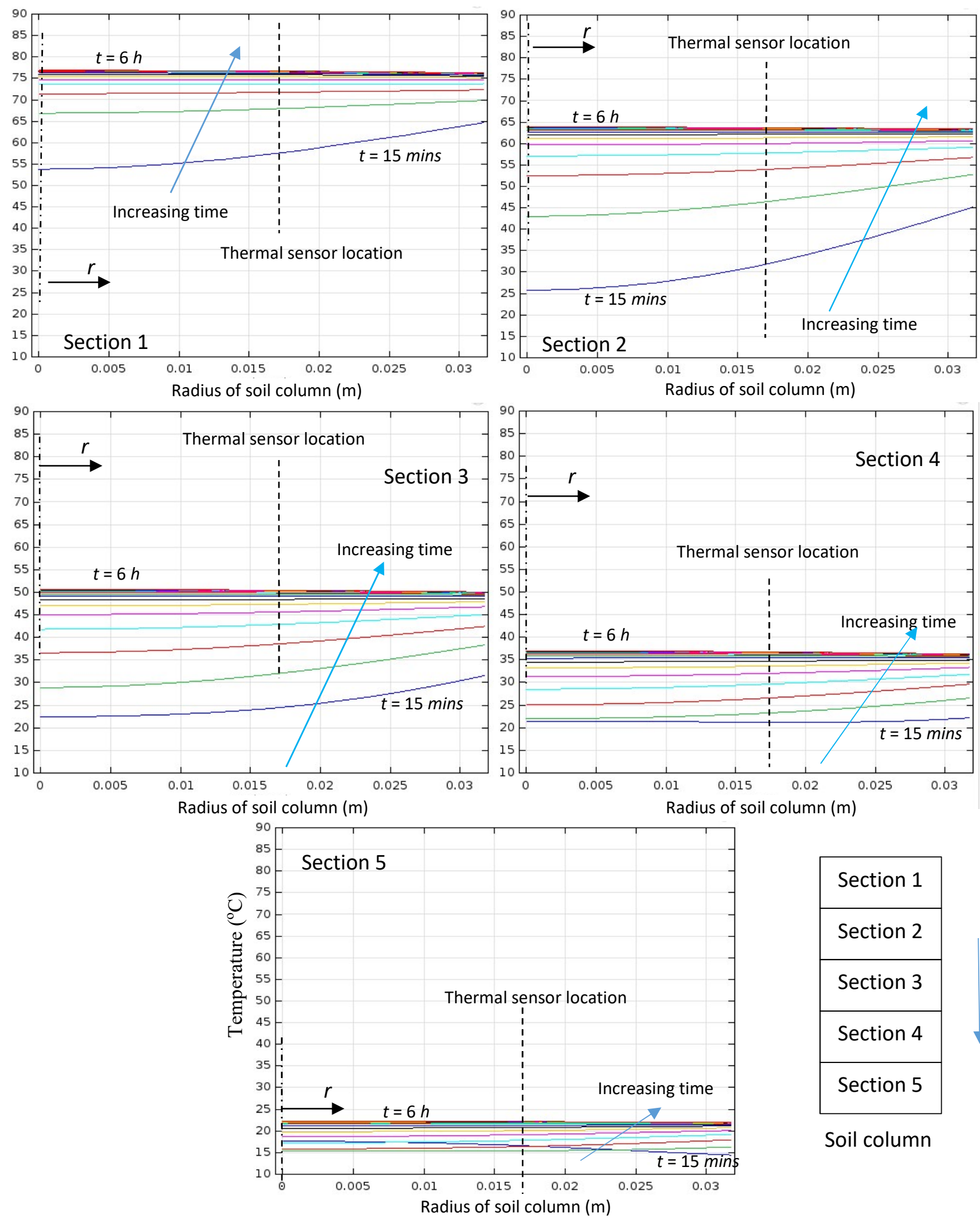

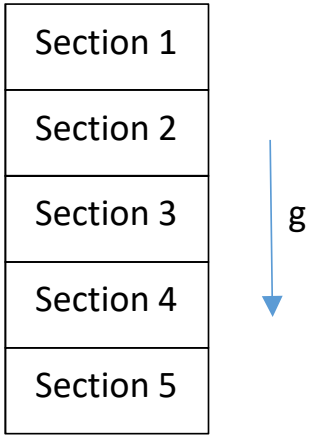

Soil column

Figure 6.8. Radial soil temperature distribution $\left({ }^{\circ} \mathrm{C}\right)$ for $S R=0.00$ in the middle of each section of the soil column from 15 minutes to 6 hours with time increment of 15 mins. 
The soil column can be split into five sections of equal volumes which correspond to the five heat pulse probes. Section 1 is on the top while section 5 is at the bottom. The radial temperature distribution of each section is further studied in Fig. 6.8 in order to examine the applicability of one-dimensional simulation using the experimental soil column. As it can be seen, the temperature in the radial direction is almost uniform after 60 minutes of heating. This means that in the first 60 minutes of heating there exists two-dimensional heat transfer in the soil column, especially in the upper hot region. The temperature at the location of the temperature sensor $($ i.e., $r=0.01675 \mathrm{~m})$ is a good representation of the average soil temperature at each cross section of the soil column. As a result, one-dimensional formulations are appropriate to numerically study the soil column after about 60 minutes into the heating.

Fig. 6.9 shows the temperature distributions along the stainless-steel tube wall of the soil column. As it can be seen, the temperature distribution along the wall of the soil column is almost linear after 10 minutes into the heating for both cases of $\mathrm{SR}=0.00$ and 0.50 . This shows that the linear temperature distribution along the wall can be established rather quickly due to the high thermal conductivity of stainless steel, as compared to the thermal conductivities of the soils and insulation. Consequently, if the axisymmetric formulations (Eq. 5.15) is to be used to study the heat and moisture transfer in the soil column, the linear temperature distribution can be assumed as the boundary condition for the soil at the interface between soil and the stainless-steel tube wall.

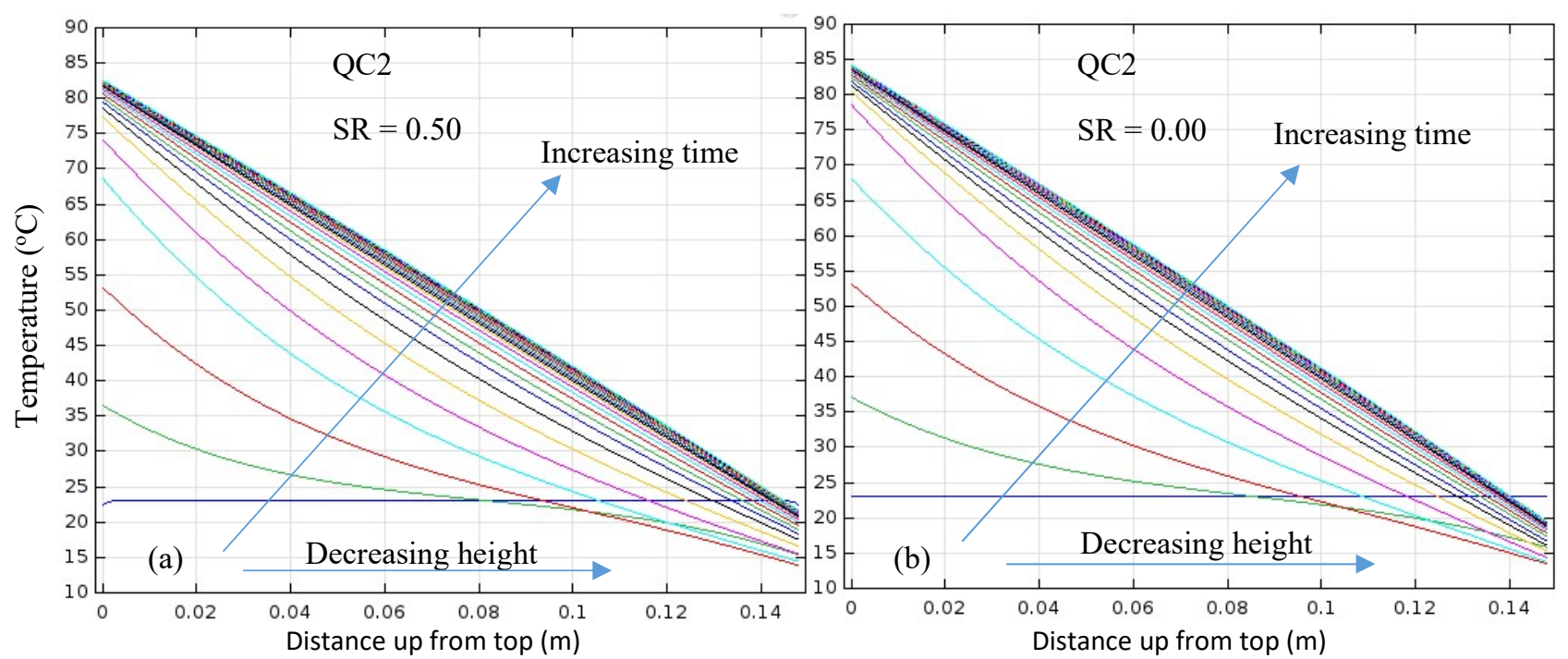

Figure 6.9. COMSOL simulated temperature distributions along the stainless-steel tube wall of the soil column for (a) $S R=0.50$ and (b) $S R=0.00$ with time increment of one minute. 


\section{3 - Numerical Simulations}

Numerical solutions of a number of cases of heat and moisture transfer using the onedimensional FVM formulations of Eq. 2.5, i.e., Eqs. 2.9a and 2.9b, for the soil column will be studied in order to verify the governing equations of coupled heat and moisture transfer in soils (Eq. 2.5) which are developed by Deru [28]. The simulated cases are based on the experimental cases. The measured temperatures at the top and bottom boundaries of the soil column are used as the prescribed boundary temperatures for all the simulations. Also, the measured initial moisture contents by the five heat pulse probes are used as the initial conditions in the simulations. Table 6.2 lists major simulation parameters used in the numerical study.

Table 6.2. Major simulation parameters [52], [53]

\begin{tabular}{|c|c|c|c|}
\hline \multirow{2}{*}{ Parameters } & \multicolumn{3}{|c|}{ Soil ID } \\
\hline & $\mathrm{BC} 1$ & NB2 & QC2 \\
\hline Porosity, $\eta\left(m^{3}\right.$ void $/ m^{3}$ soil $)$ & \multicolumn{3}{|c|}{ Dependent upon each case } \\
\hline Saturated hydraulic conductivity, $K_{\text {sat }}(\mathrm{m} / \mathrm{s})$ & $1.50 \times 10^{-5}$ & $4.01 \times 10^{-5}$ & $7.20 \times 10^{-5}$ \\
\hline Permeability, $\kappa\left(m^{2}\right)$ & $1.47 \times 10^{-12}$ & $3.93 \times 10^{-12}$ & $7.05 \times 10^{-12}$ \\
\hline $\begin{array}{l}\alpha\left(m^{-1}\right) \\
n \quad \text { in van Genuchten's models of } K \text { and } \Theta \\
\theta_{r}\left(m^{3} / m^{3}\right)\end{array}$ & $\begin{array}{l}5.82469 \\
0.81637 \\
0.60699 \\
\end{array}$ & $\begin{array}{l}0.74241 \\
1.57653 \\
0.06134 \\
\end{array}$ & $\begin{array}{r}2.62418 \\
1.21319 \\
-0.09287 \\
\end{array}$ \\
\hline Critical moisture content, $\theta_{k}\left(\mathrm{~m}^{3}\right.$ water $/ \mathrm{m}^{3}$ soil $)$ & 0.075 & 0.040 & 0.030 \\
\hline Grid size, $\Delta z(m)$ & \multicolumn{3}{|c|}{$\sim 3.33 \times 10^{-5}$} \\
\hline Time step, $\Delta t(s)$ & \multicolumn{3}{|c|}{1} \\
\hline
\end{tabular}

Note: More material properties are shown in Appendix A.

Before the simulations will be done using the developed Matlab codes, it is necessary to check and verify the codes for any potential programming mistakes or bugs. The following section will present a grid sensitivity study and verifications of the Matlab codes. 


\section{4 - Grid Sensitivity Study and Verifications of Matlab Codes}

\subsection{1 - Grid Sensitivity Study}

Because there are numerous simulation cases for three different soils with different initial moisture contents and positions of the soil column, NB2 soil with $\mathrm{SR}=0.50$ is chosen as a representative for the grid sensitivity study. Figure 6.10 shows a graph of the percentage rootmean-square differences of temperature $(T)$ and moisture content $(\theta)$ vs. number of nodes at time of 30 minutes with a simulation time-step of $1 s$ in the case of heating top of the soil column to $90^{\circ} \mathrm{C}$ and cooling the bottom to $10^{\circ} \mathrm{C}$. The percentage root-mean-square difference (\% RMS Difference) is defined as:

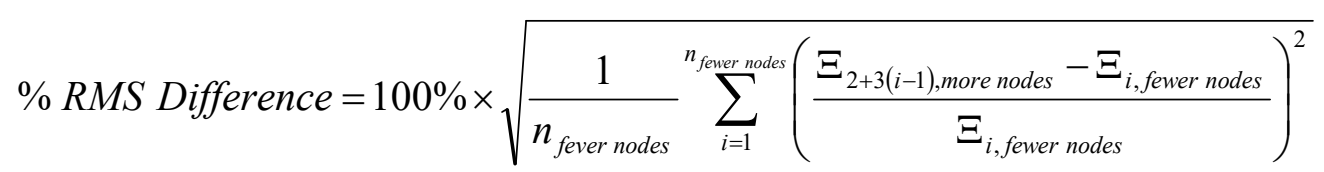

where $\Xi_{i}$ represents $T_{i}$ or $\theta_{i}$ at node $i$ and $n_{\text {fewer nodes }}$ is the total number of finite volumes or nodes in the case of fewer nodes. The successive increase of nodes is by tripling, i.e. more nodes $=$ $3 \times($ fewer nodes $)$, so that the same height points between the case of fewer nodes and the case of more nodes can be compared directly, i.e., node number of $2+3 \times(i-1)$ in the case of more nodes has the same position as node number $i$ in the case of fewer nodes.

From Fig. 6.10, it can be seen that, as the number of nodes increases, the successive percentage RMS differences of $T$ and $\theta$ become smaller, which means that the values of $T$ and $\theta$ are converging toward an infinite-node solution. Due to limited computing power, memory and time constraint, it is decided to use 4437 finite volumes for the rest of the numerical study. For this number of finite volumes, the percentage RMS differences of $T$ and $\theta$ between 4000 and 6000 nodes are less than $1.1 \%$, and the size of each finite volume is about $0.0333 \mathrm{~mm}$. 


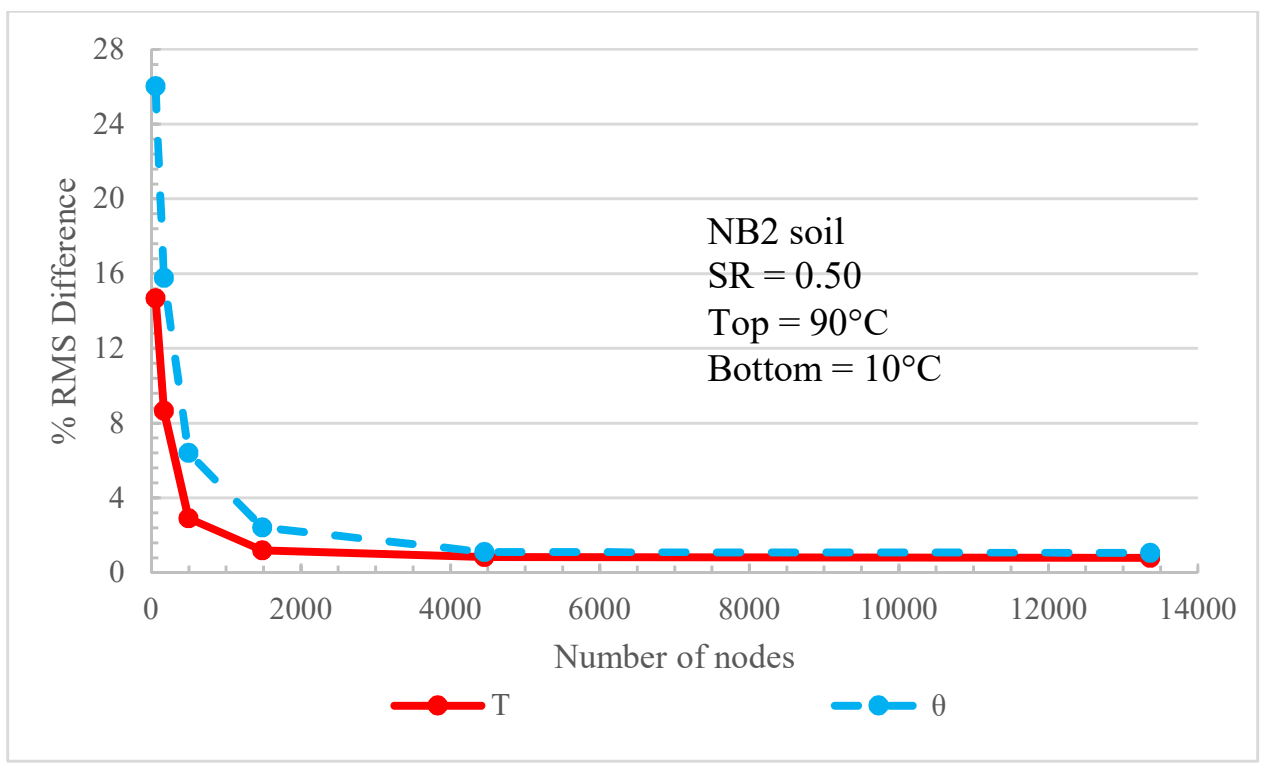

Figure 6.10. Percentage RMS differences of $T$ and $\theta v$ s. number of nodes.

\subsection{2 - Verifications of the Matlab Codes}

In order to ensure that the Matlab algorithms are correctly programmed, the one-dimensional formulations (Eq. 2.5) is numerically verified using analytical solutions for two cases: temperature distributions in a dry soil and moisture distributions in an isothermal soil. In each case, either $T$ or $\theta$ is relatively constant, so Eq. 2.5 was simplified accordingly to reach faster solutions.

\section{Case 1: Temperature distribution in a dry soil $(\theta=0.0)$}

A soil column (assumed to be semi-infinite) of height of $2 \mathrm{~m}$, density of $2,000 \mathrm{~kg} / \mathrm{m}^{3}$, thermal conductivity of $2.51 \mathrm{~W} / \mathrm{m} \cdot \mathrm{K}$, heat capacity of $837.2 \mathrm{~J} / \mathrm{kg} \cdot \mathrm{K}$, porosity of 0.50 , and initial uniform temperature $293 \mathrm{~K}$ are used. The top surface temperature of the soil is suddenly raised to $310 \mathrm{~K}$ (or $37^{\circ} \mathrm{C}$ ) at time $t=0$ and held constant. The numerical simulation is used to predict the temperature distribution in the soil after 1, 4, 9, 16, and 25 hours. The simulation time-step used is $1 s$. The results obtained are compared to the analytical solution of transient pure conduction in a semi-infinite wall [64], assuming the heat flows in the vertical direction only. The derivation of the analytical solution assumes constant thermal properties, so Case 1 is limited to small temperature difference of $\Delta T=310-293=17 \mathrm{~K}$ to reduce the effect of temperature on the thermal properties. 
Fig. 6.11 shows the temperature distributions of the semi-infinite soil column at different times from the numerical model (i.e., Eq. 2.5) and the analytical solution. As shown in Fig. 6.11, the numerical solution (or the Matlab codes) matches with the analytical solution.

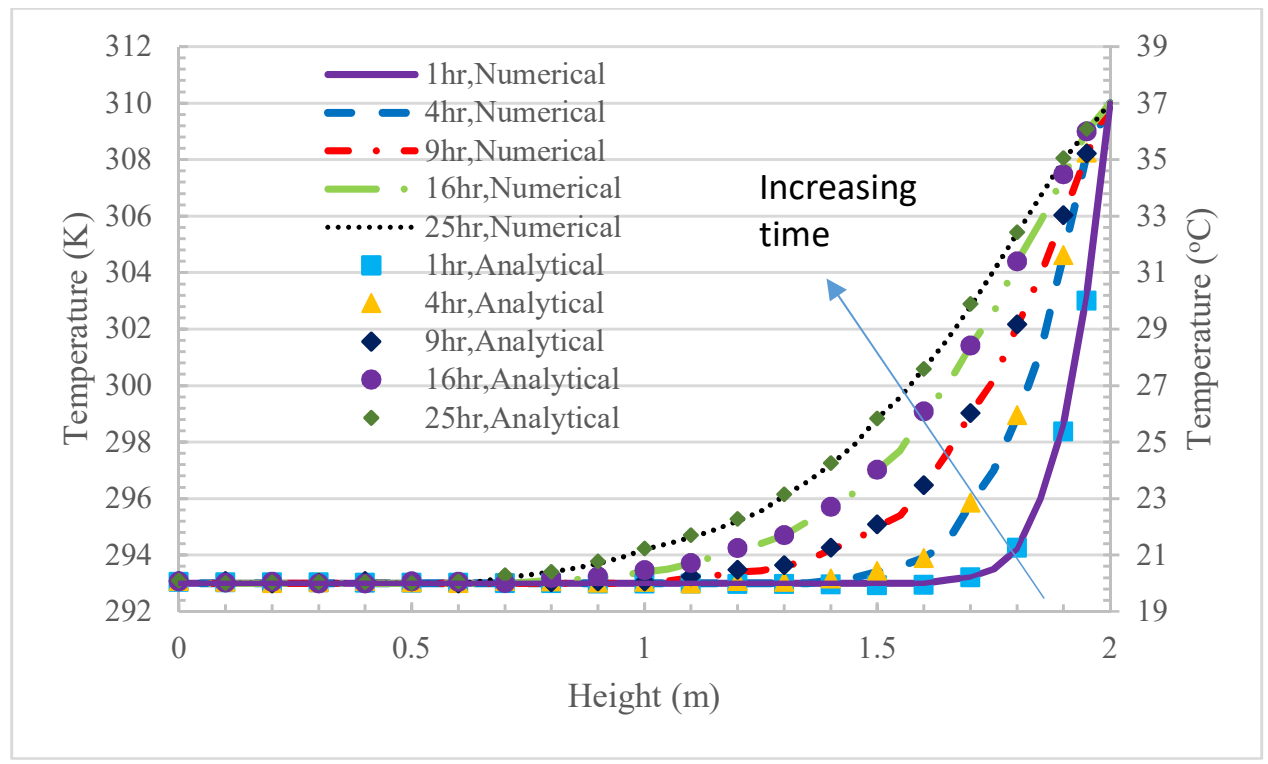

Figure 6.11. Temperature distribution of semi-infinite soil column at different times using numerical and analytical solutions.

\section{Case 2: Moisture distribution in an isothermal soil ( $T=$ constant $)$}

A column (0.4 $\mathrm{m}$ high, assumed semi-infinite) of Yolo light clay soil is used to study the moisture behavior in the soil under isothermal condition at the room temperature. The soil initially is not fully saturated and subjected to an inflow of liquid water at the top surface such that the matric potential at the top surface (i.e., $z=40 \mathrm{~cm}$ ) is always constant at 0 or $-25 \mathrm{~cm}$. The matric potential at the bottom surface $($ i.e., $z=0 \mathrm{~cm}$ ) is always held constant at $-600 \mathrm{~cm}$. Neglecting the vapor and thermal effects, the governing equation in one dimension can be written as [65]:

$$
\left(\frac{\partial \theta}{\partial \psi}\right) \frac{\partial \psi}{\partial t}=\frac{\partial}{\partial z}\left(K \frac{\partial \psi}{\partial z}\right)+\frac{\partial K}{\partial z}
$$

The initial and boundary conditions for Case 2 are: $\psi(0 s, z)=-6 m, \psi(t, 0.40 m)=0 m$ or $-0.25 m$, and $\psi(t, 0 m)=-6 m$.

The moisture retention curve and the hydraulic conductivity are given by [66]: 


$$
\begin{gathered}
\theta_{l}=\left\{\begin{array}{lr}
0.124+\frac{274.2}{739+[\ln (-\psi)]^{4}} & \psi<-1 \mathrm{~cm} \\
0.495 & \psi \geq-1 \mathrm{~cm}
\end{array}\right. \\
K=K_{s} \frac{124.6}{124.6+(-\psi)^{4}} \quad K_{s}=1.27 \times 10^{-7} \mathrm{~m} / \mathrm{s}
\end{gathered}
$$

Fig. 6.12 shows the numerical and analytical (from Philip [65]) solutions of moisture distributions at four different times of $10^{3} \mathrm{~s}(16.7 \mathrm{mins}), 10^{4} \mathrm{~s}(2.8 \mathrm{hrs}), 4 \times 10^{4} \mathrm{~s}(11.1 \mathrm{hrs})$ and $10^{5}$ $s(27.8 \mathrm{hrs})$ after moisture is first added at the top surface of the soil. As shown in the figure, when the top surface is fully saturated (i.e., $\psi$ is zero) or has a pool of water (i.e., $\psi$ is positive), more moisture can infiltrate into deeper places of the soil column. In addition, the water slowly infiltrates through the soil as clay is a fine soil which has low moisture permeability. Again, the numerical solution (or the Matlab codes) matches very well with the analytical solution.
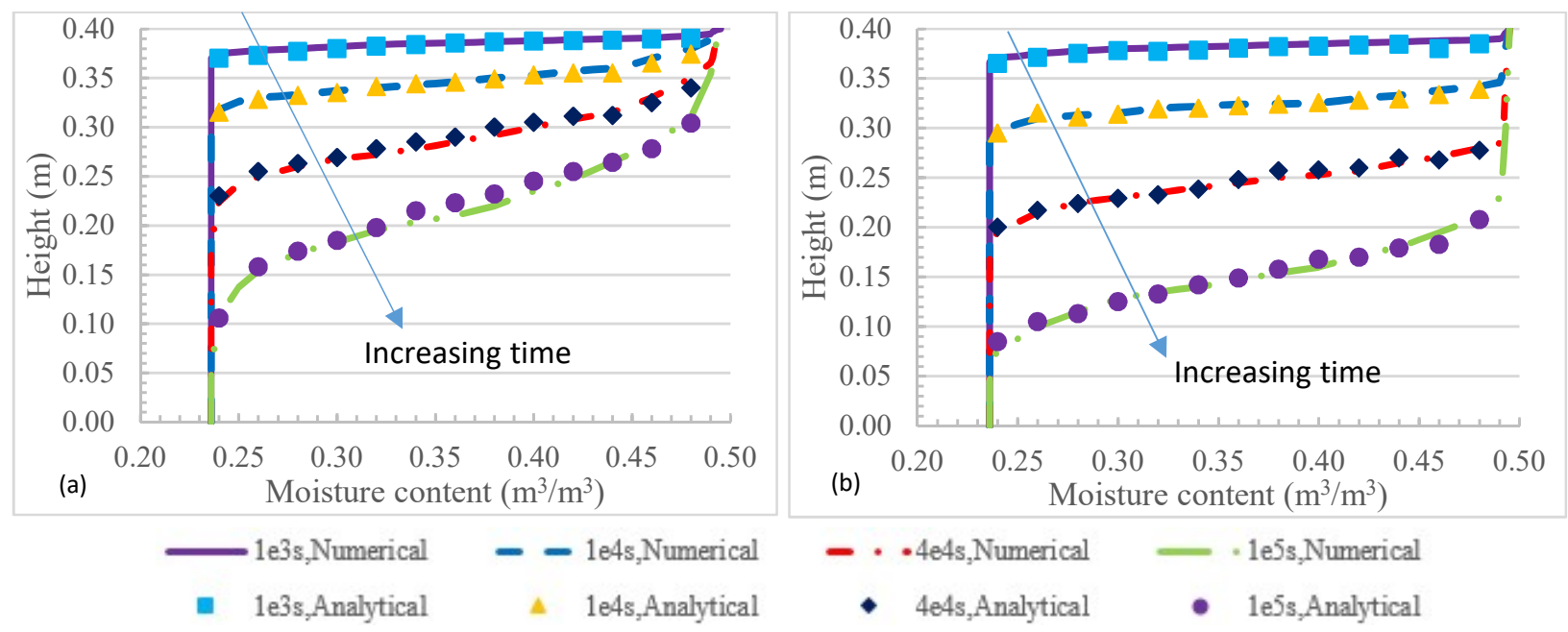

Figure 6.12. Moisture distributions in Yolo light clay soil column from numerical and analytical solutions for $(a) \psi(t, 40 \mathrm{~cm})=0 \mathrm{~cm}$ and $(b) \psi(t, 40 \mathrm{~cm})=-25 \mathrm{~cm}$.

Fig. 6.12 shows the moisture behaviors (at different times after moisture is first added at the top surface of the soil) in the Yolo light clay soil column using numerical and analytical (from Philip [65]) solutions. As shown in the figure, when more moisture is added at the top surface (i.e, $\psi$ is less negative), more moisture can infiltrate into deeper places of the soil column. In addition, the moisture (or water) slowly infiltrate through the soil as clay is a fine soil. 
From the two cases studied, the Matlab codes is considered to be verified and sufficient for further numerical studies. In the next section, numerical simulations of different heating and orientation conditions are studied according to the experimental cases. One-dimensional FVM (finite volume method) formulations are applied for the simulations. The temperature readings from the experimental heat flux meters (HFMs) are used as the boundary temperatures.

From the two cases studied, the Matlab codes is considered to be verified and sufficient for further numerical studies. In the next section, numerical simulations of different heating and orientation conditions are studied according to the experimental cases. One-dimensional FVM (finite volume method) formulations are applied for the simulations. The temperature readings from the experimental heat flux meters (HFMs) are used as the boundary temperatures.

\section{5 - Sample Numerical Solutions}

\subsection{1-BC1 Soil with $S R=0.00$}

In this case, dry $\mathrm{BC} 1$ soil with $\eta=0.59$ is simulated based on the parameters listed in Table 6.2. Since moisture involvement in dry soils does not exist, the moisture content is set to be zero. However, both heat and moisture equations are still applied and solved.

Fig. 6.13 shows the simulated temperature at each needle inside the soil column (with dry $\mathrm{BC} 1$ soil) which is vertically heated to $90^{\circ} \mathrm{C}$ at the top plate and cooled to $10^{\circ} \mathrm{C}$ at the bottom plate from room temperature. The letter N1 in the legends means the needle number 1 and so on. The highest and lowest lines are the measured temperatures at the top and bottom of the soil column and are used as the prescribed temperature boundary conditions. Even though the water baths were set to thermally control the water at $90^{\circ} \mathrm{C}$ and $10^{\circ} \mathrm{C}$ each, the temperatures at the top and bottom boundaries of the soil column cannot reach $90^{\circ} \mathrm{C}$ and $10^{\circ} \mathrm{C}$, respectively, even at steady state. The first reason is heat loss/gain from/to the water hoses. The second reason is the overall thermal resistances between the circulating water in the flow channels inside the aluminum plates and the heat flux meters at the boundaries of the soil column. The third reason is the thermal responses of the soil column during experimentation. 


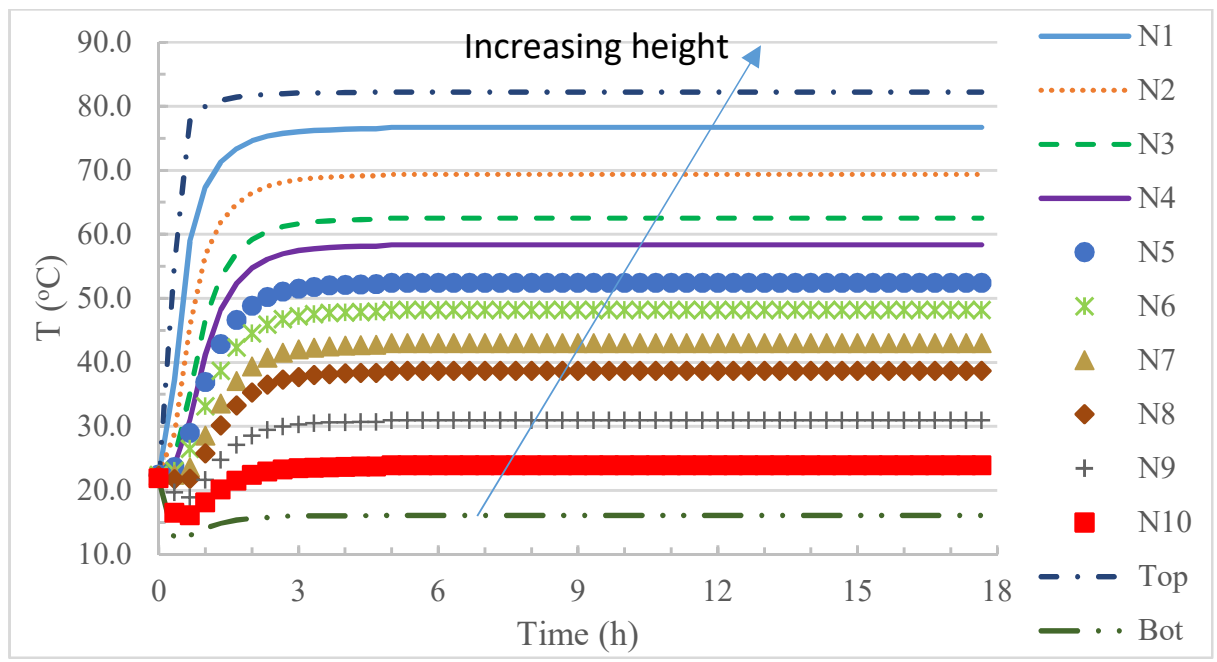

Figure 6.13. Simulated temperature at each needle (N\#) for the case of dry $B C 1(\eta=$ $0.59)$ and vertical heating from top.

Fig. 6.14 shows the soil column (dry BC1) being heated from the bottom plate and cooled at the top plate. Similar to the previous case shown in Fig. 6.13, the boundary temperatures cannot reach $90^{\circ} \mathrm{C}$ and $10^{\circ} \mathrm{C}$. In both cases, the soil column reaches steady state after about four to five hours. Comparing between Fig. 6.13 and Fig. 6.14, the thermal responses of the two cases are similar, except that the heating-from-the-bottom case (Fig. 6.14) has overall higher temperatures than the heating-from-the-top case (Fig. 6.13) by about $4^{\circ} \mathrm{C}$. One reason can be from the weight of the soil causing the bottom portion to be more compact (or lower porosity) than the top portion of the soil column. Since thermal properties depend on the porosity, as shown in Chapter 4, the results are different between the heating-from-the-top and heating-from-the-bottom cases. Another reason is from applying the insulation to the soil cell. Ideally, the insulation should be equally spread around stay put to the soil cell. However, during the installation of the wooden box (in Fig. 3.2) to encase the soil cell and the insulation, non-uniform air space can form between insulation pieces. In addition, insulation could not be applied uniformly around the soil cell due to the soil cell's configuration. The third reason is from the physical behaviors of heating from the top and from the bottom. When the bottom of the soil column is heated, the hotter air can move upward to higher spaces through the pores inside the soil due to buoyancy effect and heat is transferred more through the soil. On the other hand, the top heating case is stable and the hotter air remains at the top instead of moving down, resulting in heat from the hot top plate being conducted more through the stainless-steel tube wall and the outer container of the soil. 


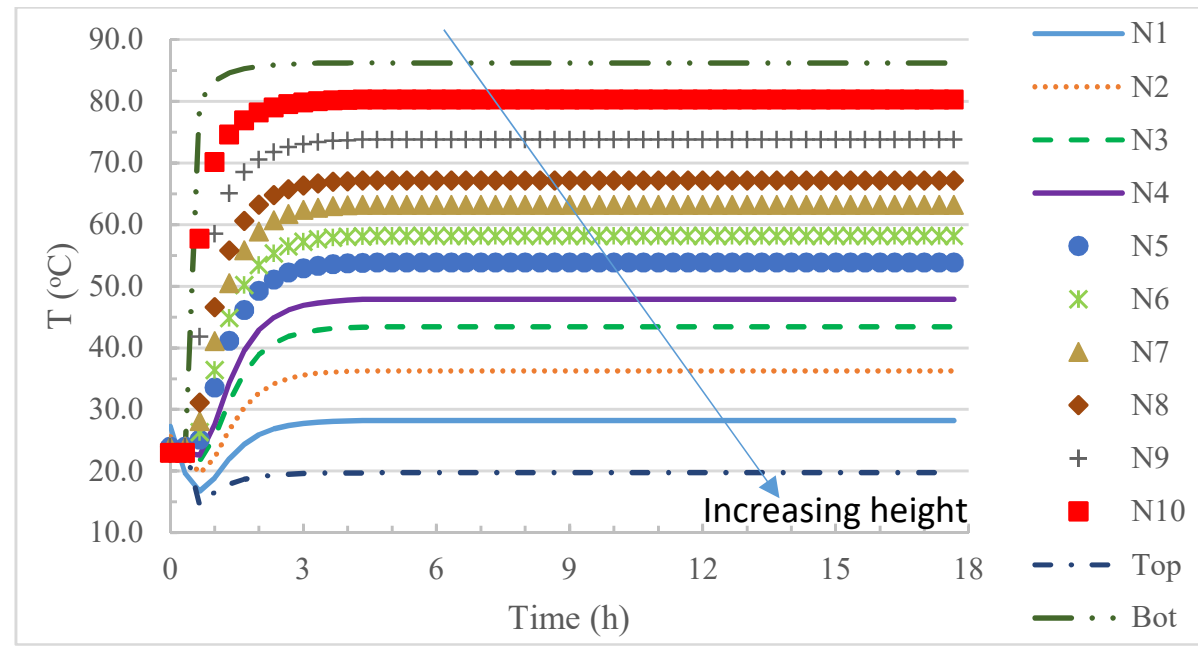

Figure 6.14. Simulated temperature at each needle (N\#) for the case of dry $B C 1(\eta=$ $0.59)$ and vertical heating from bottom.

Fig. 6.15 shows the simulated temperature at each needle when the soil cell is heated horizontally. The hot plate in the case of Fig. 6.15 is the hot plate in the case of Fig. 6.13. The temperatures at the same needle locations in Fig. 6.15 are slightly higher than those in Fig. 6.13 by about $0.5^{\circ} \mathrm{C}$. Due to the horizontal orientation of the soil cell during the experimentation, there exists a temperature gradient perpendicular to the gravity and hence the hot air can move more freely towards the colder regions and more heat can be transferred in the needle locations.

The temperature responses for dry NB2 and QC2 soils are similar to the ones for BC1 soil and can be seen in Appendix E. 


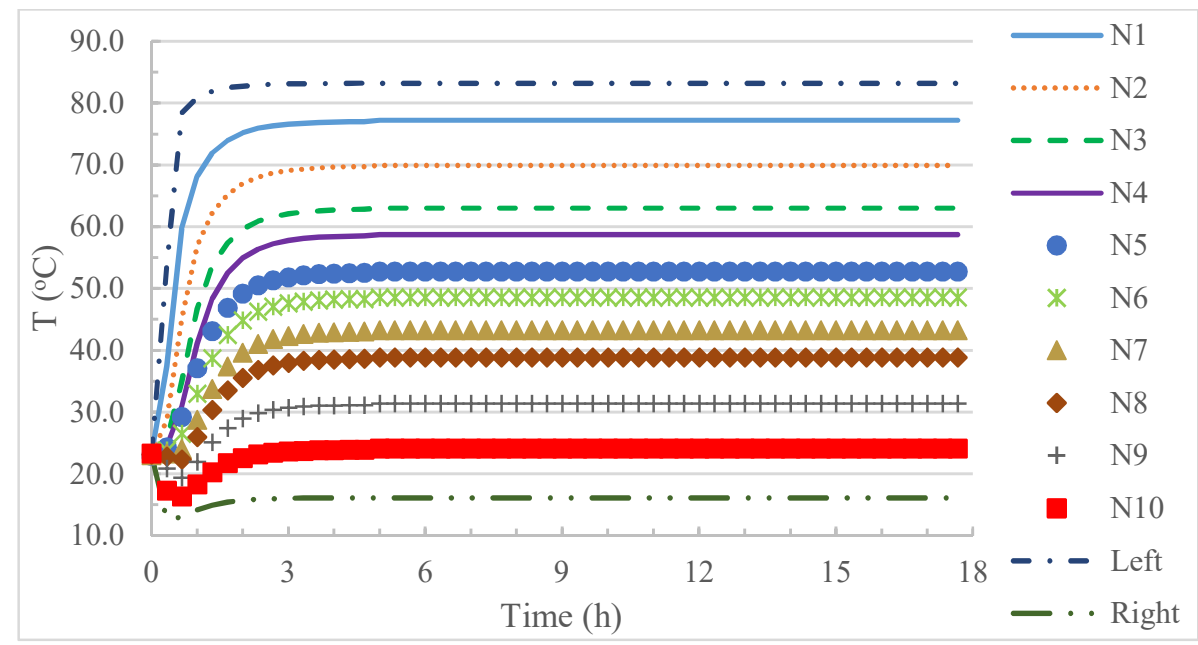

Figure 6.15. Simulated temperature at each needle (N\#) for the case of dry $B C 1(\eta=$ $0.59)$ and horizontal heating.

\subsection{2-BC1 Soil with $S R=0.25$}

In this case, wet $\mathrm{BC} 1$ soil at $\mathrm{SR} \approx 0.25$ is simulated based on the parameters listed in Table 6.2. Since there is moisture transfer in this case, both heat and moisture equations are applied and solved.

Fig. 6.16 shows the simulated temperature and moisture contents of wet $\mathrm{BC} 1(\mathrm{SR} \approx 0.25)$ with vertical heating from the top. In the temperature responses, unlike Fig. 6.13 with almost the same temperatures after four hours of heating, some locations have rising temperatures after four hours of heating while many others have dropping temperatures. The locations with the rising temperatures (the top two needles) are near the hot plate. The locations with the dropping temperatures are further from the hot plate. The reason is from the movement of the moisture in the soil. As shown in Fig. 6.16b, the moisture contents at the top two needles (N1 and N2) quickly drop due to the high thermal gradient caused by the hot top plate. After being moved by the thermal gradient for about 30 minutes, the moisture content starts to reach the critical moisture content $\theta_{k}$. When a pore space is occupied by more moisture (or simply water), the space becomes harder to be heated up due to the much higher thermal capacity of water than the one of air. Also, the moisture movements in the lower half of the soil column are less than those in the upper half as indicated in Fig. 6.16b. One reason is that the lower half has lower thermal gradients than the upper 
half. Another reason is that the lower half is colder, so the moisture is harder to move through the pore space due to higher viscosity.
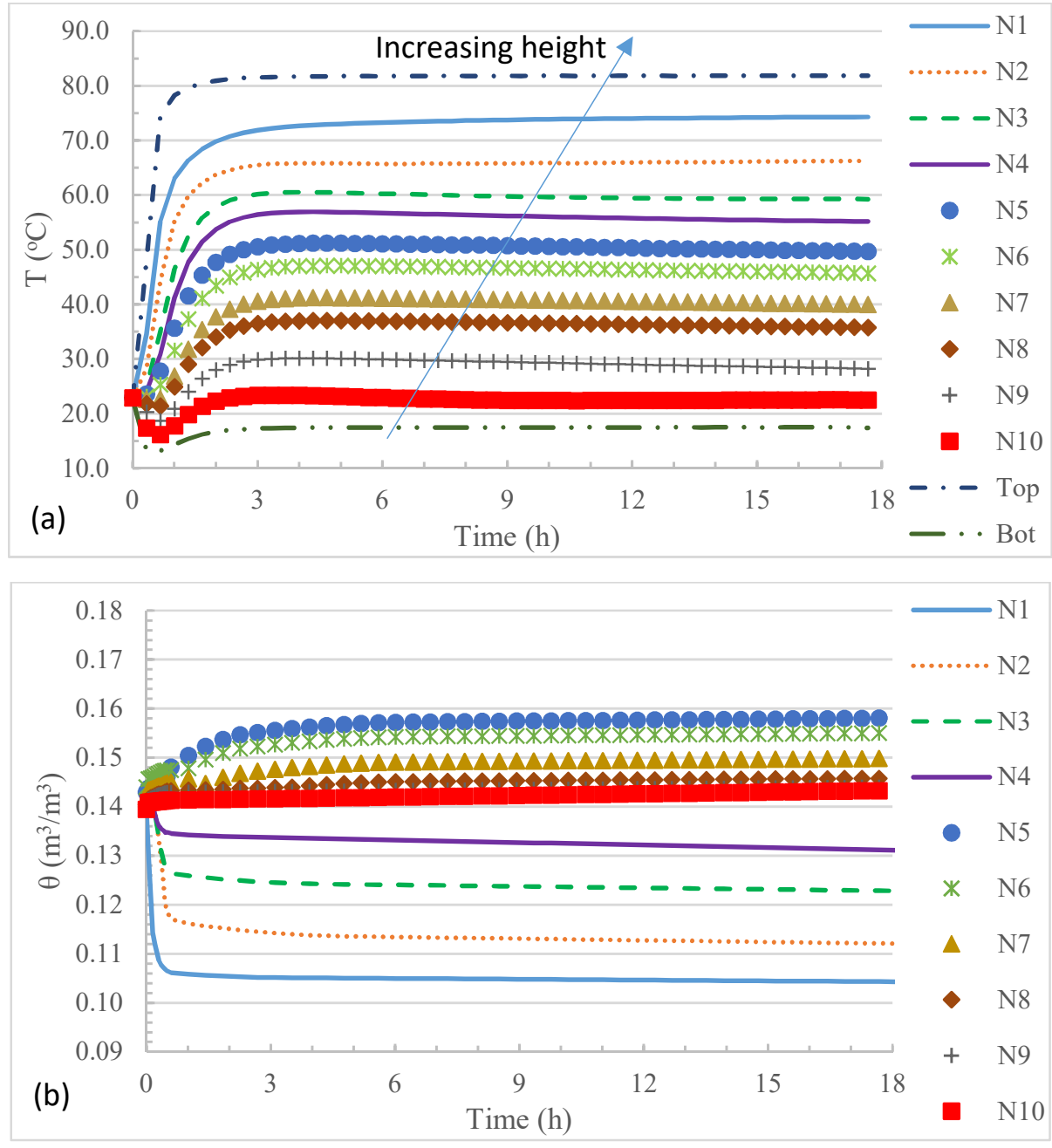

Figure 6.16. Simulated (a) temperature and (b) moisture content responses at each needle for the case of wet $B C 1(\eta=0.56, S R \approx 0.25)$ and vertical heating from top.

Fig. 6.17 shows the simulated temperature and moisture contents of wet $\mathrm{BC} 1(\mathrm{SR} \approx 0.25)$ with vertical heating from the bottom. Similar to the temperature response in Fig. 6.16, the temperatures at a location (N10) rises after four hours of heating while others drop. The reason is very similar to Fig, 6.16a. However, gravity makes the differences in the moisture contents between Fig. 6.16b and Fig. $6.17 \mathrm{~b}$. When the soil column is heated from the bottom, the moisture closes to the bottom plate moves up due to the thermal gradient. On the other hand, gravity tries to pull the moisture down. At higher temperatures, the moisture is easier to be pulled down by gravity because of lower 
viscosity of the moisture (or water). Due to these competing effects, the moisture content at N10 in Fig. 6.17 rapidly drops to the lowest value of $0.115 \mathrm{~m}^{3} / \mathrm{m}^{3}$ at time $=0.35$ hours and then slowly increases to about $0.122 \mathrm{~m}^{3} / \mathrm{m}^{3}$ after a long time. However, for the case of heating from the top in Fig. $6.16 \mathrm{~b}$, the moisture content at $\mathrm{N} 1$ rapidly drops to $0.102 \mathrm{~m}^{3} / \mathrm{m}^{3}$ at time $=0.35$ hours and then continuously drops to about $0.104 \mathrm{~m}^{3} / \mathrm{m}^{3}$ after a long time. The highest moisture content occurs at $\mathrm{N} 6$ to be about $0.173 \mathrm{~m}^{3} / \mathrm{m}^{3}$ for the heating from the bottom after a long time; whereas, the highest moisture content occurs at $\mathrm{N} 5$ to be about $0.169 \mathrm{~m}^{3} / \mathrm{m}^{3}$ for the heating from the top.

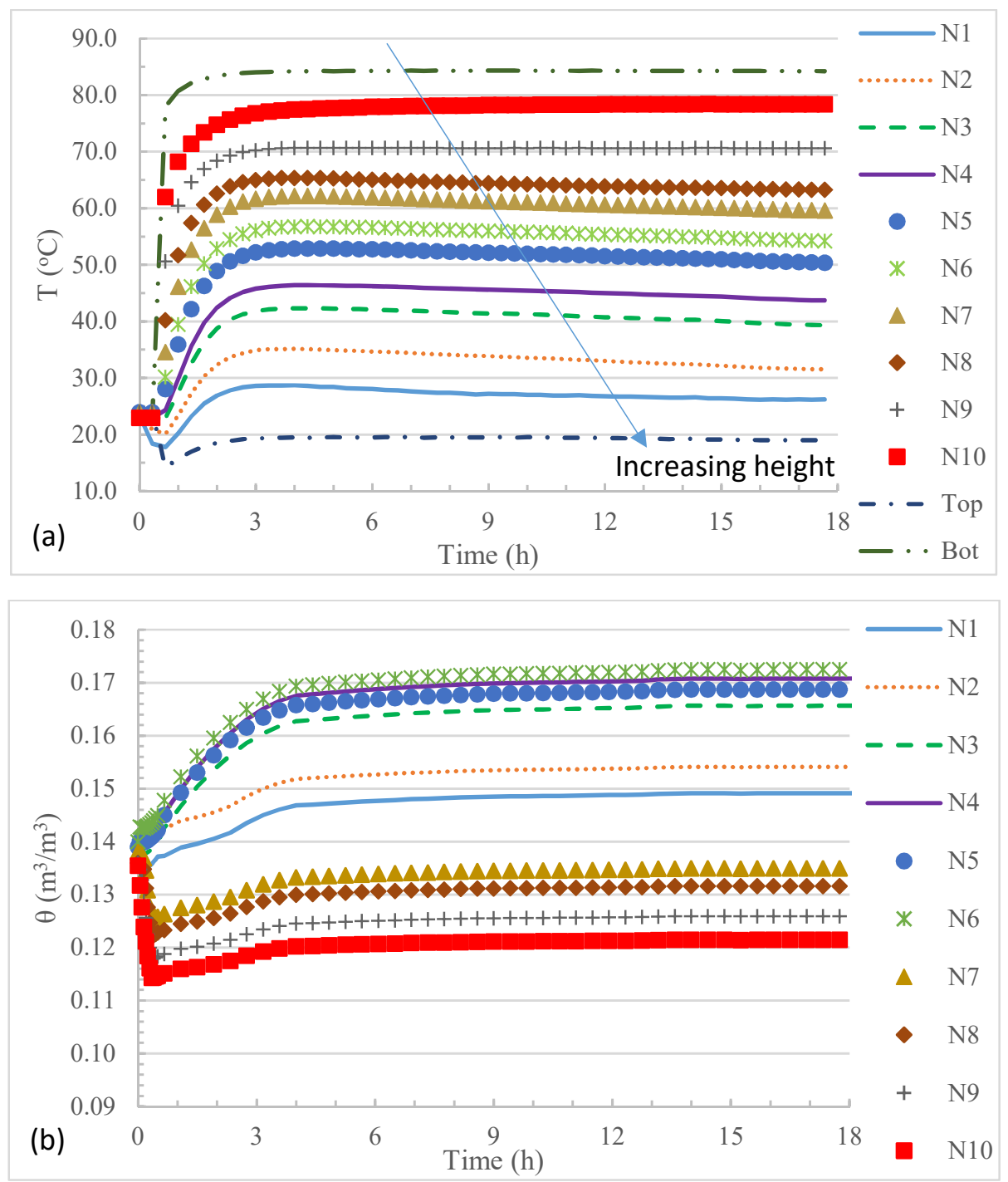

Figure 6.17. Simulated (a) temperature and (b) moisture content responses at each needle for the case of wet $B C 1(\eta=0.54, S R=0.25)$ and vertical heating from bottom. 
Fig. 6.18 shows the simulated temperature and moisture contents of wet $\mathrm{BC} 1(\mathrm{SR} \approx 0.25)$ with horizontal heating. Comparing between Fig. 6.16a and Fig. 6.18a, the temperature responses are similar up to time of about 3 hours; after that, only location N1 has very slight decreasing temperature while the others have more obvious decreasing temperatures. This is due to the decreasing colder boundary temperature from $15.7^{\circ} \mathrm{C}$ at time $=3$ hours to $13.3^{\circ} \mathrm{C}$ at time $=18$ hours, instead of holding steady at about $17.4^{\circ} \mathrm{C}$ as the case of heating from the top (Fig. 6.16a). This may be because of the wooden supporting structure of the soil cell during the horizontal heating; less heat is gained from the structure and the cold water bath is capable of continuously lowering the cold plate temperature. As a result, the colder boundary temperature after four hours of heating drops as seen in Fig. 6.18a. For vertical heating, the soil cell is supported by four steel bolts; more heat can be gained via the steel bolts than the wooden supporting structure and the cold water bath has just enough capacity to keep the cold plate temperature constant. Although there are small differences in soil temperatures between Fig. 6.16a and Fig. 6.18a due to the gradual decrease of colder boundary temperature, it is interesting to note that the moisture contents are similar between Fig. 6.16b and Fig. 6.18b.

The temperature and moisture responses for wet NB2 and QC2 soils with $\mathrm{SR} \approx 0.25$ are similar to those for $\mathrm{BC} 1$ soil and can be seen in Appendix E. Since QC2 soil is the coarsest soil among the three tested soils, its moisture content near the hot plate is the lowest. NB2 soil is finer than QC2 soil and has higher corresponding moisture content near the hot plate. But the moisture content near the hot plate is correspondingly lower than that in $\mathrm{BC} 1$ soil. 

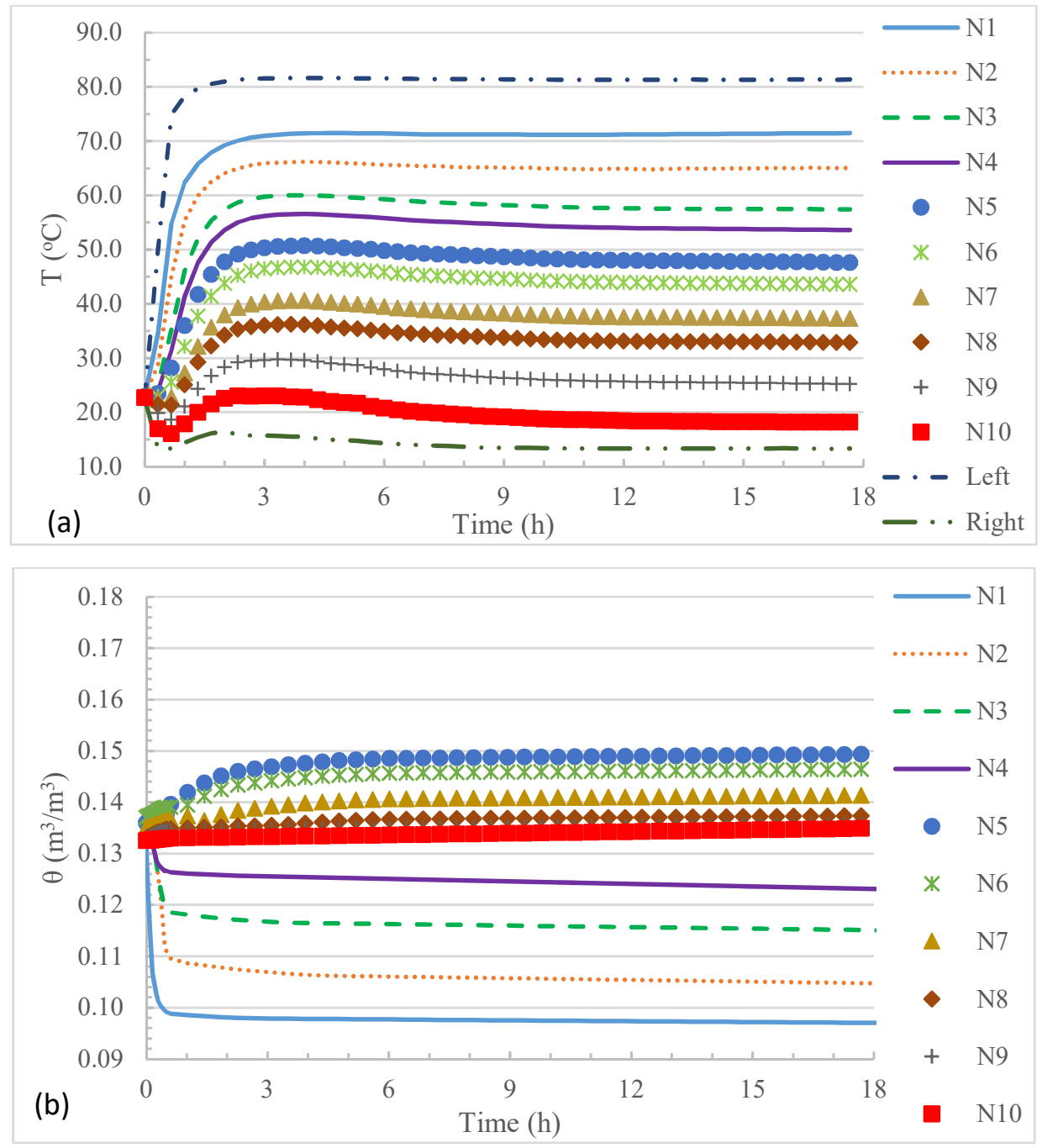

Figure 6.18. Simulated (a) temperature and (b) moisture content responses at each needle for the case of wet $B C 1(\eta=0.53, S R \approx 0.25)$ and horizontal heating.

\subsection{3-BC1 Soil with $S R=0.50$}

In this case, wet $\mathrm{BC} 1$ soil at $\mathrm{SR} \approx 0.5$ is simulated based on the parameters listed in Table 6.2. Since there is moisture transfer in this case, both heat and moisture equations are applied and solved.

Fig. 6.19 shows the simulated temperatures and moisture contents of wet $\mathrm{BC} 1(\mathrm{SR} \approx 0.50)$ with vertical heating from the top plate. The moisture content in the soil region near the hot plate drops rapidly in the first 30 minutes and then stays relatively constant afterward. Because of the good water holding capacity of $\mathrm{BC} 1$ soil (which is a fine soil), the moisture content in the soil 
region near the hot plate does not drop very low. For example, the moisture content at location N1 has the biggest drop of only about $8.6 \%$ from its initial moisture content.

The moisture contents in Fig. 6.19b are higher than those in Fig. 6.16b, so the heat is transferred more due to higher soil thermal conductivity and the boundary temperatures in Fig. 6.19a are lower than those in Fig. 6.16a. In general, Fig. 6.19a is similar to Fig. 6.16a, except at locations N1 and N2 the temperatures drop gradually after time $=3$ hours. This may be due to heat being conducted away as a result of higher soil thermal conductivity (because of higher moisture content). When the soil is vertically heated up from the top, moisture moves down because of the thermal gradient from the top. After a certain time, as the viscosity starts to balance the thermal gradient and the gravity, the moisture stops moving further down. At this time, the moisture (or water) begins to absorb heat and lowers the temperature.

When the soil is vertically heated up from the top, moisture moves down because of the thermal gradient from the top. After a certain time, as the viscous and capillary forces start to balance the driving forces of the thermal gradient and the gravity, the moisture stops moving further down. At this time, due to little transfer of moisture (or water), the heat conduction begins to be more prominent and heat is being conducted away due to higher soil thermal conductivity, resulting in gradual decrease of the temperature. 

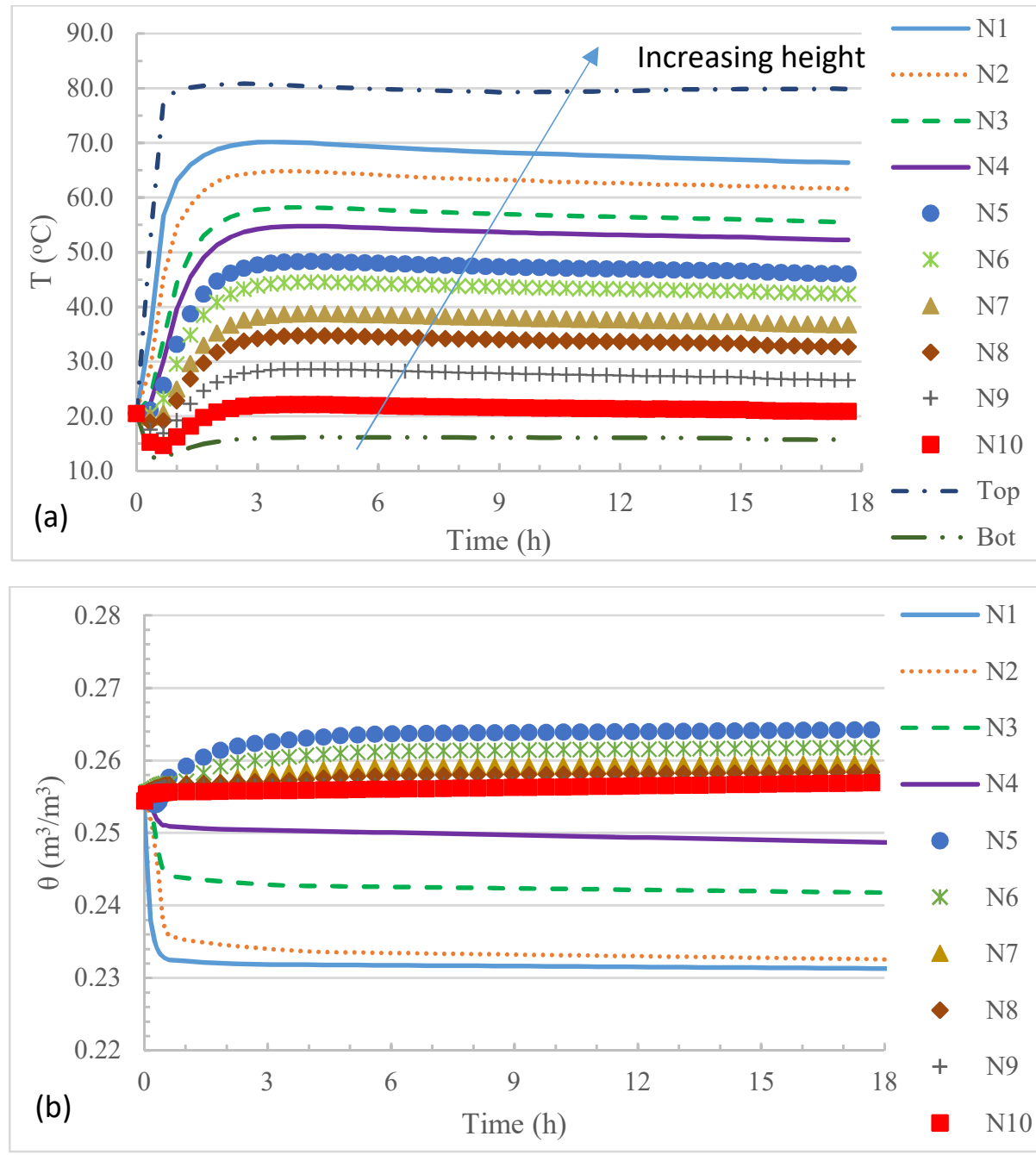

Figure 6.19. Simulated (a) temperature and (b) moisture content responses at each needle for the case of wet $B C 1(\eta=0.51, S R=0.50)$ and vertical heating from top.

Fig. 6.20 shows the simulated temperatures and moisture contents of wet $\mathrm{BC} 1(\mathrm{SR} \approx 0.50)$ with vertical heating from the bottom plate. The trend is similar to Fig. 6.17, except for temperatures at locations $\mathrm{N} 9$ and N10. It seems that the gradual decrease of temperatures at locations N9 and N10 after time $=3$ hours is related to the higher soil thermal conductivity (as a result of higher moisture content, i.e., $0.244 \mathrm{~m}^{3} / \mathrm{m}^{3}$ vs. $0.122 \mathrm{~m}^{3} / \mathrm{m}^{3}$ of Fig. $6.17 \mathrm{~b}$ ). 

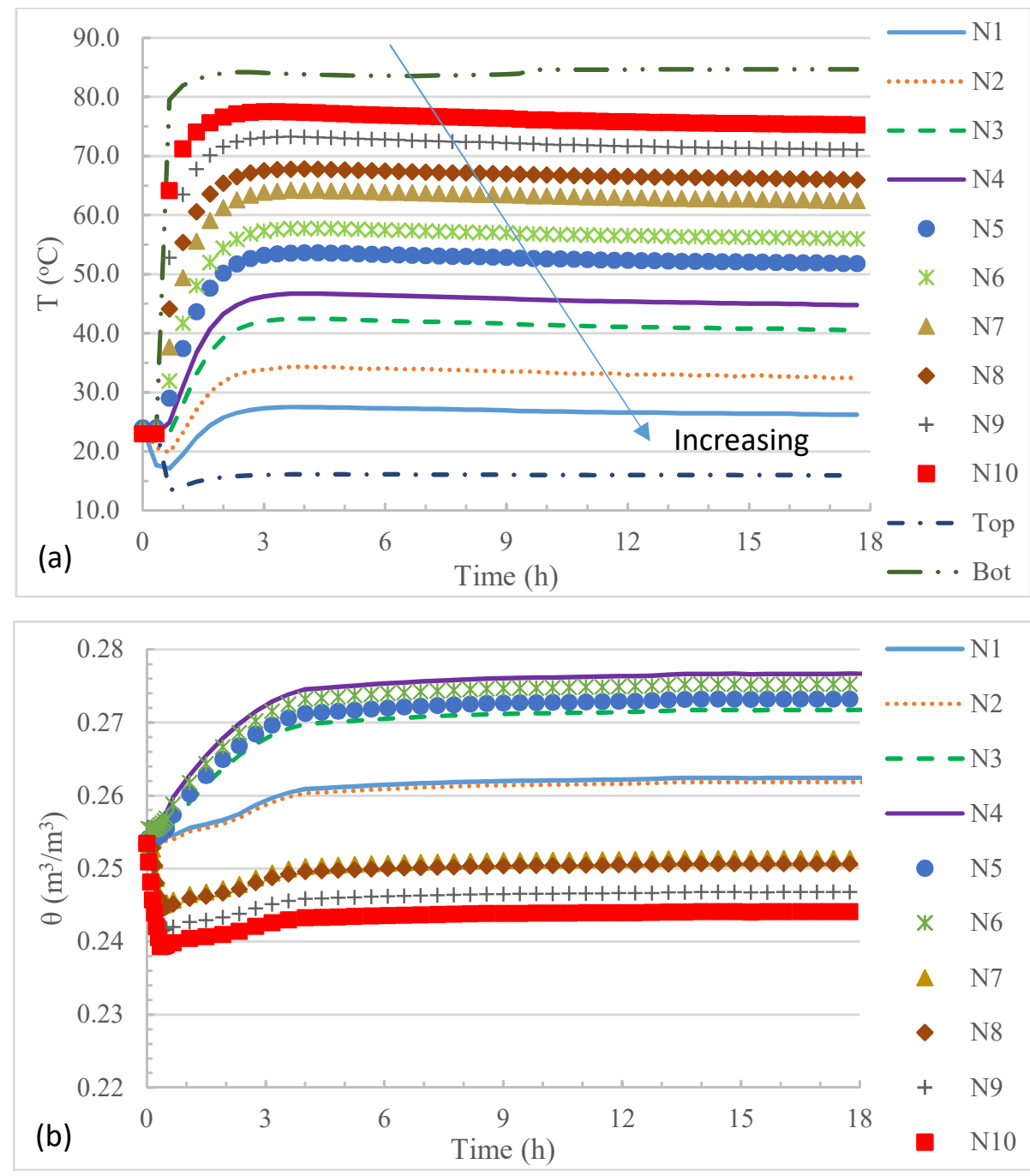

Figure 6.20. Simulated (a) temperature and (b) moisture content responses at each needle for the case of wet BCl $(\eta=0.51, S R=0.50)$ and vertical heating from bottom.

Fig. 6.21 shows the simulated temperatures and moisture contents of wet $\mathrm{BC} 1(\mathrm{SR} \approx 0.50)$ with horizontal heating. The trend is similar to Figs. 6.18 and 6.20.

The temperature and moisture responses for wet $\mathrm{NB} 2$ and QC2 with $\mathrm{SR} \approx 0.50$ soils are similar to those for BC1 soil and can be seen in Appendix E. Because QC2 soil is the coarsest soil, it has the highest drop in moisture content near the hot plate. NB2 soil is finer than QC2 soil, so the moisture content does not drop as much in NB2 soil as in QC2 soil but still has higher drop than in $\mathrm{BC} 1$ soil. 

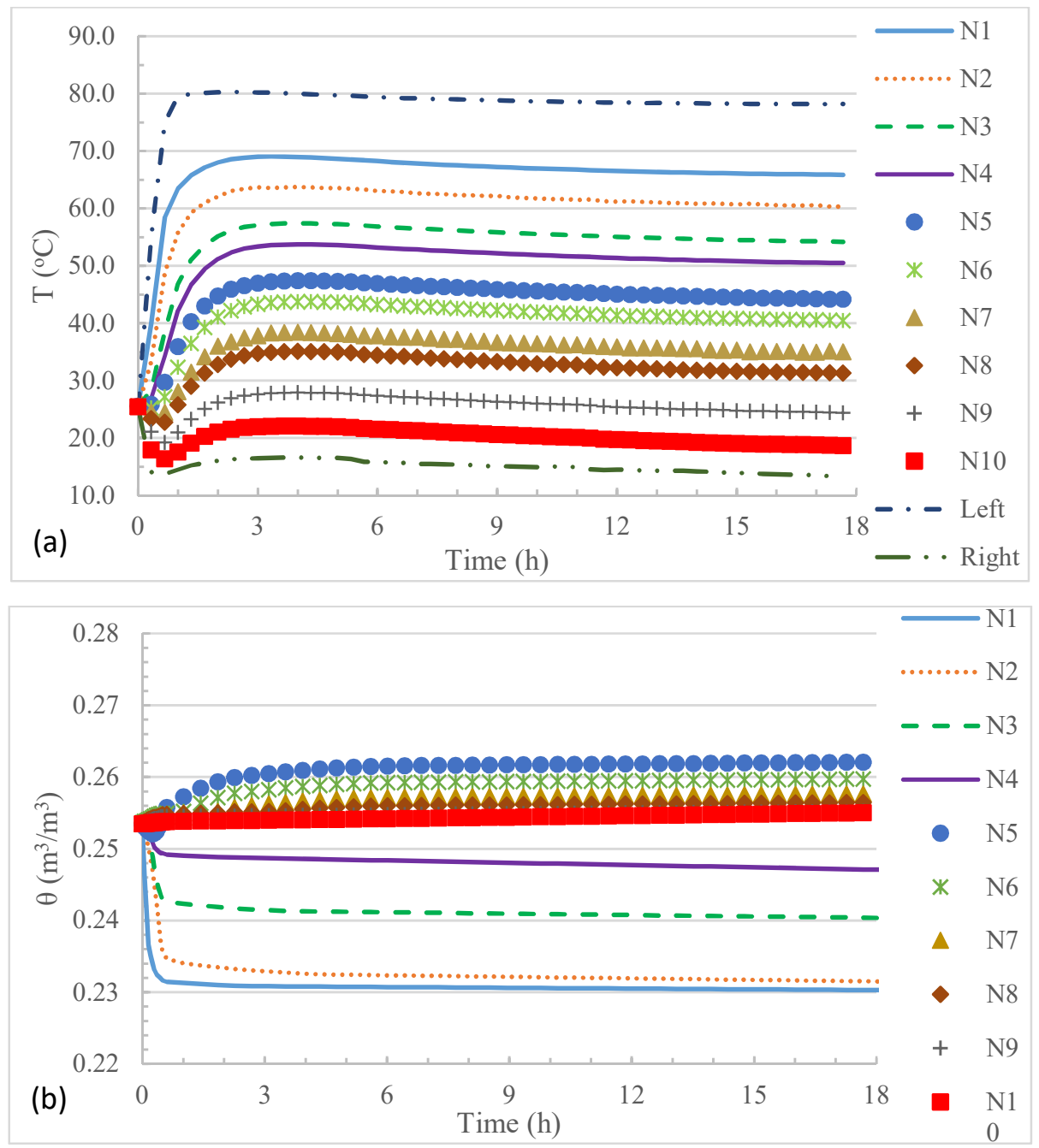

Figure 6.21. Simulated (a) temperature and (b) moisture content at each needle for the case of wet BC1 $(\eta=0.51, S R=0.50)$ and horizontal heating.

\section{$6.6-$ Summary}

In this chapter, different heating conditions of $\mathrm{BC} 1, \mathrm{NB} 2$, and $\mathrm{QC} 2$ soils with different saturation ratios are being simulated based on the one-dimensional FVM formulations. A soil can be either vertically heated to $90^{\circ} \mathrm{C}$ at the top and cooled to $10^{\circ} \mathrm{C}$ at the bottom, vertically heated to $90^{\circ} \mathrm{C}$ at the bottom and cooled to $10^{\circ} \mathrm{C}$ at the top, or horizontally heated to $90^{\circ} \mathrm{C}$ and cooled to $10^{\circ} \mathrm{C}$. The boundaries temperatures at the top and bottom of the soil column are taken from the recorded temperatures of the heat flux meters during experimentations. Because of the heat 
loss/gain to/from the room from/to the water hoses, the insulation, and the thermal resistances between water in the flow channels in the aluminum plates and the heat flux meters, the boundary temperatures cannot be $90^{\circ} \mathrm{C}$ and $10^{\circ} \mathrm{C}$ as set in the water baths during the experimentations. Due to the limited computing power, memory and time, the soil column cannot have a very large amount of nodes in the simulations. It is found from the grid sensitivity study that 4439 nodes (corresponding to 4437 control volumes) is sufficient for the simulations of coupled heat and moisture transfer in the soil column.

For a dry soil (either BC1, NB2, or QC2), vertical heating from the top produces lower boundary temperatures than vertical heating from the bottom and horizontal heating do. One reason may be attributed to the weight of the soil causing variation of porosity and hence thermal properties between the top and bottom portions of the soil column. Another reason comes from the application of the insulation. Because of the heating conditions and orientations of the soil cell during experimentations, more heat escapes from the hot plate of the soil cell when the cell is being heated vertically from the top. The third reason is from the physical behaviors of the heating conditions. When the soil cell is vertically heated from the top, the hot air tends to stay on the top under the buoyancy effects, so less heat is conducted downwards through the soil. Moreover, the temperature at a location in the soil column is almost constant after four hours of heating and cooling.

For a wet soil, unlike in the dry soil, the temperature after four hours of heating and cooling at a location drops and then increases depending on how much moisture (or water) there is in the soil. Initially, when the soil column is heated, the moisture near the hot plate moves away from the plate because of the thermal gradient. After a certain time, when the viscous and capillary forces start to balance the driving forces of thermal gradient and gravity, the moisture migration starts to slow down and eventually becomes still. Due to the variation of soil thermal conductivity, as a result of the variation of moisture content, there is a net heat being conducted away, resulting in a gradual temperature decrease. However, if a soil is coarse (QC2) or medium (NB2) with low moisture content $(\mathrm{SR}<0.25)$, some moisture migration toward the colder region of the soil column would still exist; eventually the soil thermal conductivity would be low enough to reduce heat conduction toward the colder region. In this situation, the heat conduction from the hot plate starts to raise up again the temperature at the hot region near the hot plate. 
The next chapter will show more simulated results and compare them with the experimental data. Enlarged and more detailed graphs in this chapter are shown in Appendix E. 


\section{CHAPTER 7 - VERIFICATION OF THE THEORETICAL MODEL OF HEAT AND MOISTURE TRANSFER IN SOILS}

\section{1 - Pure Conduction Heat Transfer}

In this section, the pure conduction heat transfer model using COMSOL will be compared with the experimental data. The whole soil cell is simulated in axisymmetric geometry similar to Section 6.2. The material properties, other than $k$ and $C_{p}$ of the soil, are the same as in Section 6.2. The soil's $k$ and $C_{p}$ for $\mathrm{SR} \approx 0.50$ follow Sections 4.2 and 4.3 (i.e., thermal properties as functions of temperature). The soil's $k$ and $C_{p}$ for $\mathrm{SR} \approx 0.25$ are described in Appendix A.8. The COMSOL module used is the built-in pure conduction model instead of the porous media model as shown in Section 6.2.

\subsection{1-BC1 soil}

Fig. 7.1 shows the measured heat fluxes and compares the simulated temperatures and heat fluxes (from pure conduction model using COMSOL) with the measured data. The measured heat fluxes by the heat flux meters are at the top and bottom of the soil column while the top and bottom temperatures are at the locations (respectively) of the highest and lowest needles of the heat pulse probes inside the soil column. The needles are $8.79 \mathrm{~mm}$ from the boundaries. In Fig. 7.1b, the left vertical axis shows the percentage errors between the simulated temperatures and the measured data while the vertical right axis shows the percentage errors between the simulated and the measured heat fluxes by the heat flux meters (HFMs). The percentage error is defined as:

$$
\% \text { Error }=100 \% \times \frac{\Xi_{\text {experiment }}-\Xi_{\text {simulation }}}{\Xi_{\text {experiment }}}
$$

where $\Xi$ can represent the heat flux (in $W / m^{2}$ ) or temperature (in ${ }^{\circ} \mathrm{C}$ ).

In Fig. 7.1b, pure conduction heat transfer model in COMSOL is used to simulate the experimental apparatus. The soil column is vertically heated from the top plate at $90^{\circ} \mathrm{C}$ and cooled at the bottom plate at $10^{\circ} \mathrm{C}$. From the figure, the simulated temperatures at the top and bottom needles are relatively close to the measurements (within $2.5 \%$ error). However, the simulated heat 
fluxes at the top and bottom of the soil column are far from the recorded values during experimentation (up to $22.9 \%$ error). The reason is that the pure heat conduction model (PHCM) does not account for the moisture and air movement in the soil. Consequently, the model (pure conduction) gives steady-state results after three hours of heating and the temperatures and heat fluxes inside the soil column are relatively constant after that time. Nonetheless, the effects from moisture do not stop after heating for three hours around which time the moisture migrates more slowly. After more moisture moves away from the top region of soil near the hot plate, the soil thermal conductivity is lower and the heat flux drops. Therefore, after about 0.5 hours of heating, the measured heat flux at the hot plate starts to drastically drop. It is interesting that the simple PHCM can produce good results for soil temperature, while it is not good in predicting the heat transfer.
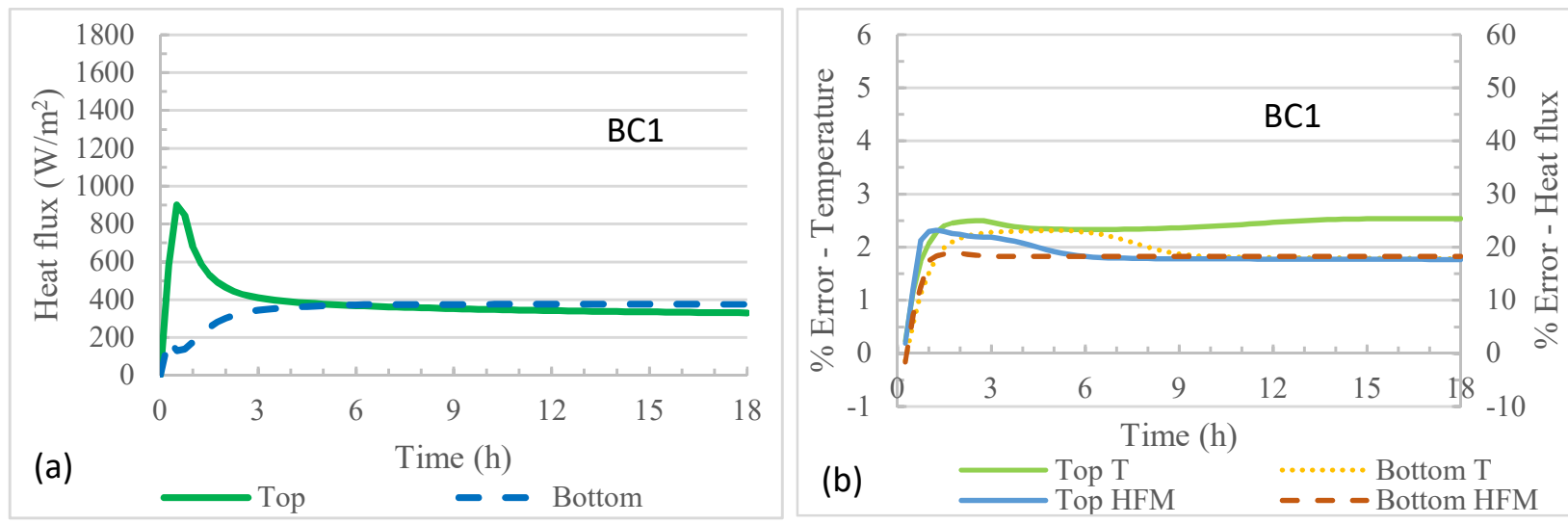

Figure 7.1. $B C 1$ soil ( $\eta=0.56, S R=0.25)$ for the case of vertically heated from top: (a) measured heat fluxes and (b) \% errors in temperatures and heat fluxes.

Fig. 7.2 is similar to Fig. 7.1, but the soil column is vertically heated from the bottom. Ideally speaking, the top and bottom HFMs should measure the same values at long time (after three hours of heating) during experimentation. However, in reality, some of the heat from the stainless-steel tube of the soil column flows in to the colder region of the soil near the top. In addition, some of the heat from the stainless-steel tube evaporates moisture at around the tube-soil interface in the hotter region near the bottom and middle of the soil column; then the water vapour migrates upward due to concentration gradient and buoyancy and condenses in the colder region releasing latent heat of vaporization. Therefore, the top HFM senses much more heat transfer than the bottom HFM (the hot side in this case) does. 

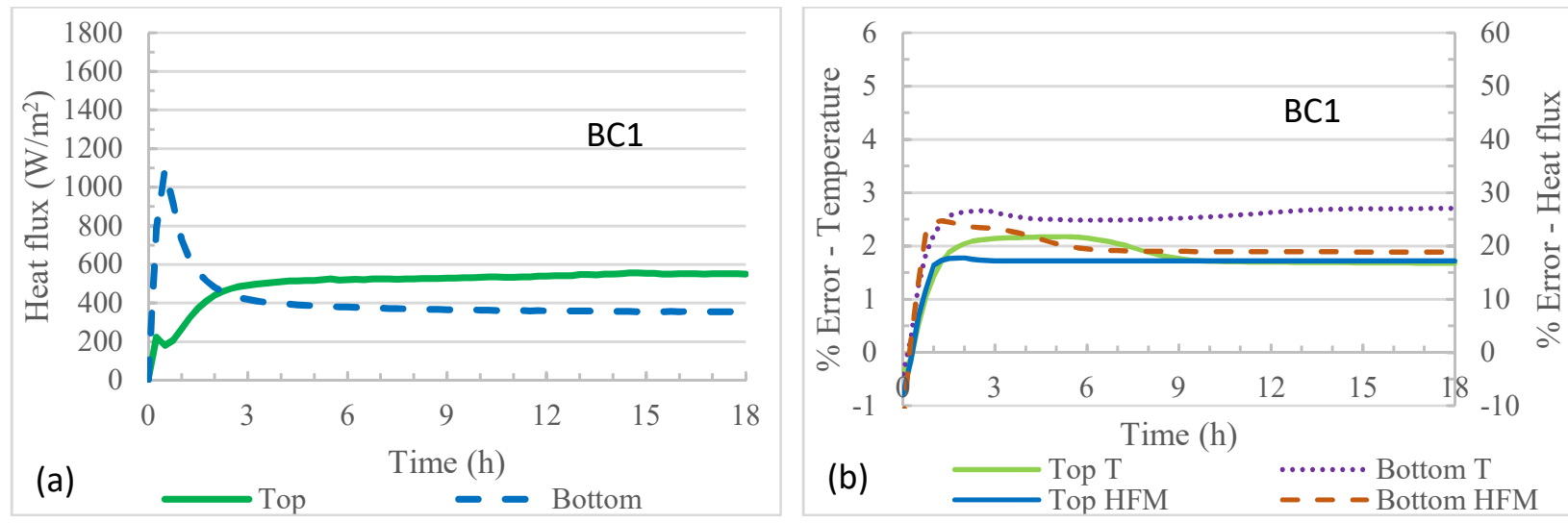

Figure 7.2. $B C 1$ soil ( $\eta=0.54, S R=0.25)$ for the case of vertically heated from bottom: (a) measured heat fluxes and (b) \% errors in temperatures and heat fluxes.

The trend in Fig. 7.2b is similar to that in Fig. 7.1b. Generally speaking, the errors from the cold side are lower than the errors from the hot side because there is less moisture movement in the cold region than that in the hot region. Moreover, the errors from the bottom heating is slightly higher than that from the top heating.

Fig. 7.3 is based on the same experimental setup as Fig. 7.1, but the soil column is horizontally heated. The trend in Fig. 7.3 is similar to that in Fig. 7.1. However, as mentioned in Section 6.5.1, the hot air and water vapour in horizontal heating can circulate towards the cold side by natural convection than that in vertical heating from the top. While circulating toward the cold plate, the air and water vapour can gain more heat from the stainless-steel tube wall. As mentioned above, when the water vapour reaches the cold region, it condenses and releases latent heat of vaporization. So the cold-side HFM (indicated as Bottom in Fig. 7.3a) senses higher heat flux than the one in Fig. 7.1a.

Fig. 7.4 is based on similar experimental setup as Fig. 7.1, except that the soil in Fig. 7.4 has more moisture (around field capacity, i.e. $\mathrm{SR} \approx 0.50$ ) than the soil in Fig. 7.1. The trend in Fig. 7.4 is very similar to the one in Fig. 7.1. Due to more moisture content and higher soil thermal conductivity, the heat from the hot side can be transferred more to the cold side. As a result, the HFMs in Fig. 7.4 show higher values than the ones in Fig. 7.1 do. In addition, more moisture content in the soil means less void space for the water vapour to move around. As a result, the errors for the simulation in Fig. 7.4b are less than those in Fig. 7.1b. This indicates that if there is 
lower vapour migration, the PHCM would be more accurate in predicting the heat transfer; but the errors are still relatively high at around $15 \%$ after a long time.

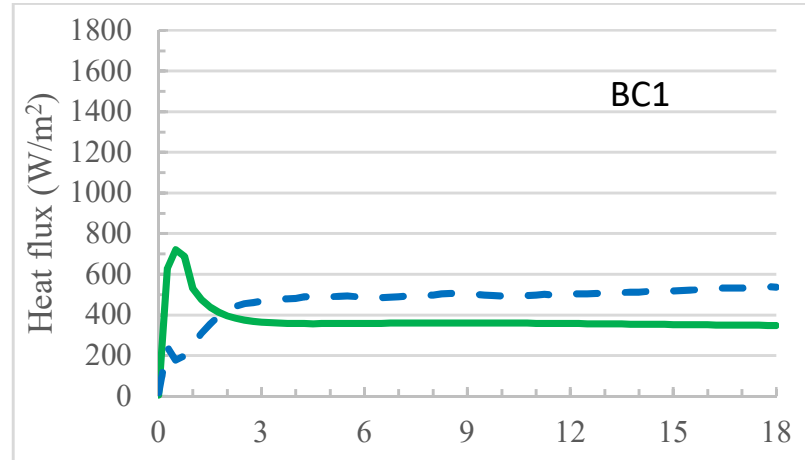

(a)

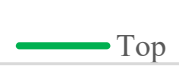

Time (h)

- Bottom

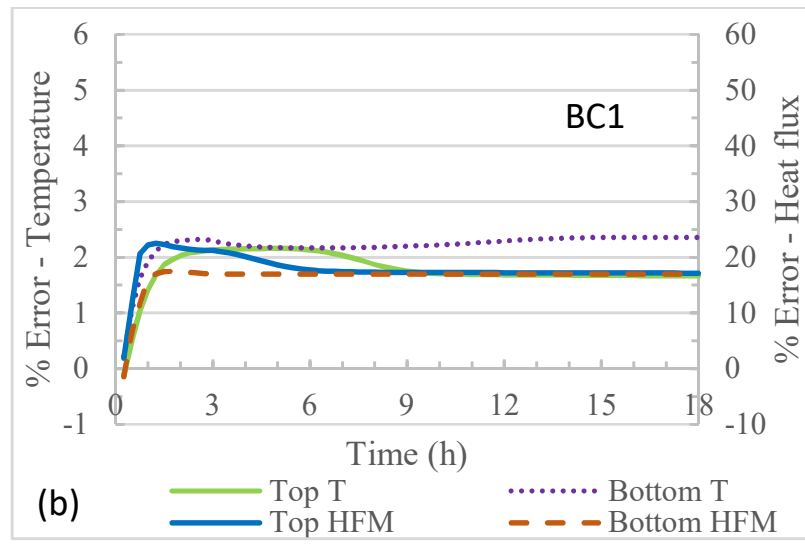

- - Bottom HFM

Figure 7.3. BC1 soil $(\eta=0.53, S R=0.25)$ for the case of horizontally heated: (a) measured heat fluxes and (b) \% errors in temperatures and heat fluxes.

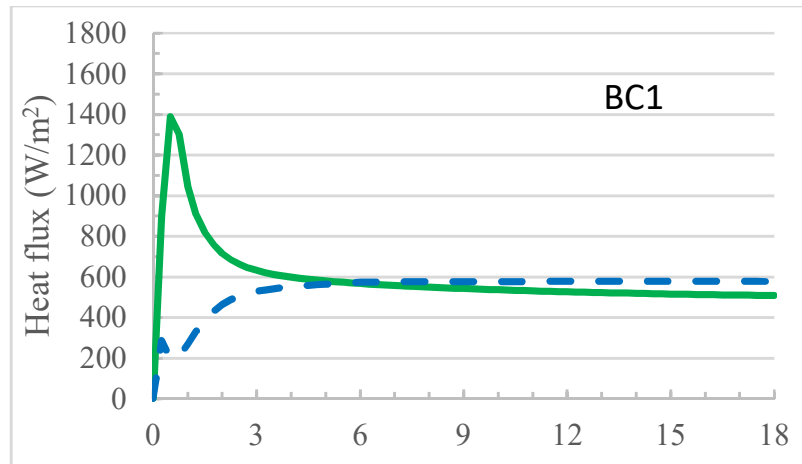

(a)

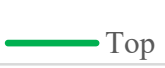

Time (h)

- Bottom

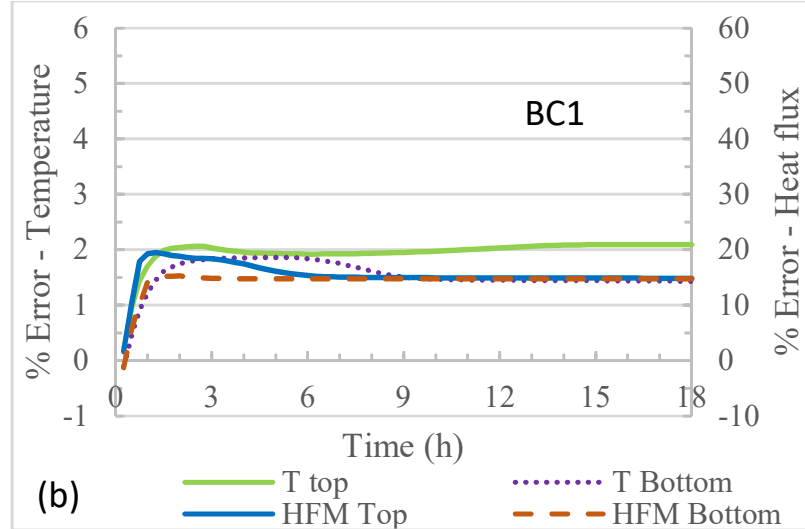

(b)

Figure 7.4. BC1 soil $(\eta=0.51, S R=0.50)$ for the case of vertically heated from top: (a) measured heat fluxes and (b) \% errors in temperatures and heat fluxes.

Figs. 7.5 and 7.6 are respectively similar to Figs. 7.2 and 7.3, but the soil in the column has more moisture content (around field capacity, i.e. $\mathrm{SR} \approx 0.50$ ). Because of higher moisture contents, the wetter soils can transfer more heat from the hot side to the cold side, so the HFMs measure higher heat fluxes. It is very interesting to see that the top heat flux is twice the bottom heat flux after a long time in Fig. 7.5a. Due to higher moisture content, more evaporation can happen near tube-soil interface and also easier for the condensed moisture to return from the top region due to gravity and smaller matric potential. 

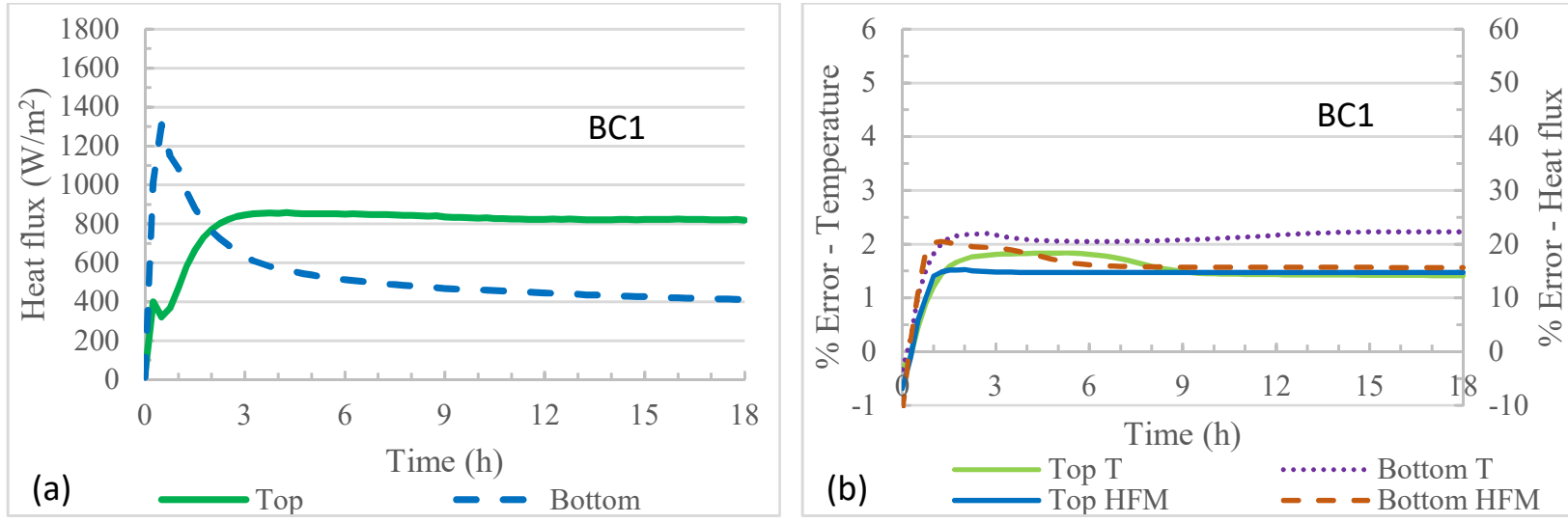

Figure 7.5. BC1 soil ( $\eta=0.51, S R=0.50)$ for the case of vertically heated from bottom: (a) measured heat fluxes and (b) \% errors in temperatures and heat fluxes.
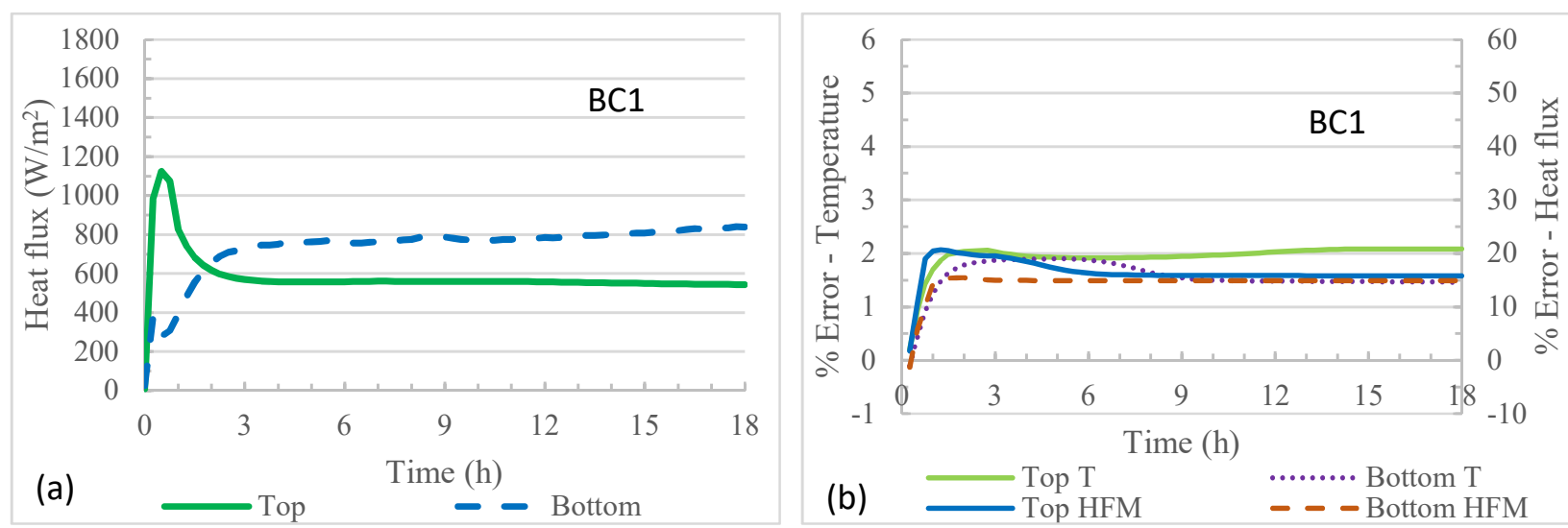

Figure 7.6. BC1 soil $(\eta=0.51, S R=0.50)$ for the case of horizontally heated: (a) measured heat fluxes and (b) \% errors in temperatures and heat fluxes.

\subsection{2 - NB2 Soil}

Figs. $7.7-7.12$ show the measured heat fluxes and comparisons of the PHCM simulations with the experimental values for wet NB2 soils under different testing conditions. The trend in each figure is similar to the corresponding trend for BC1 soil. Moreover, looking at the HFM of the hot side in part (a) of each figure, it can be noted that maximum heat flux for NB2 soil is higher than the corresponding heat flux for $\mathrm{BC} 1$ soil. In other words, under transient conditions, more heat can be transferred through NB2 soil than BC1 soil with the same saturation ratio. The reason is that NB2 soil has higher thermal conductivity than $\mathrm{BC} 1$ soil has. On the other hand, at long time (after 15 hours of heating), the heat flux through NB2 soil is less than the one through BC1 soil. The reason is that NB2 soil is coarser than BC1 soil is. Consequently, after a long heating time, 
more moisture can migrate away from the hot region and less heat can be transferred through the soil column due to lower soil thermal conductivity in the hot region. In terms of simulation errors of the PHCM, NB2 soil has higher errors of simulated temperatures and heat fluxes in part (b) of each figure than $\mathrm{BC} 1$ soil has. This indicates that when there is more moisture migration, resulting in greater variation of moisture content in NB2 soil, the PHCM is less accurate in simulating heat transfer through the soil column. Under this situation, it is necessary to simulate with coupled heat and moisture transfer in the soil, as later presented in Section 7.4. For temperature, the highest error is $5.5 \%$ in Fig. $7.9 \mathrm{~b}$ which is acceptable; however, the highest error for heat flux is $37.4 \%$ in Fig. $7.9 \mathrm{~b}$ which is unacceptable.
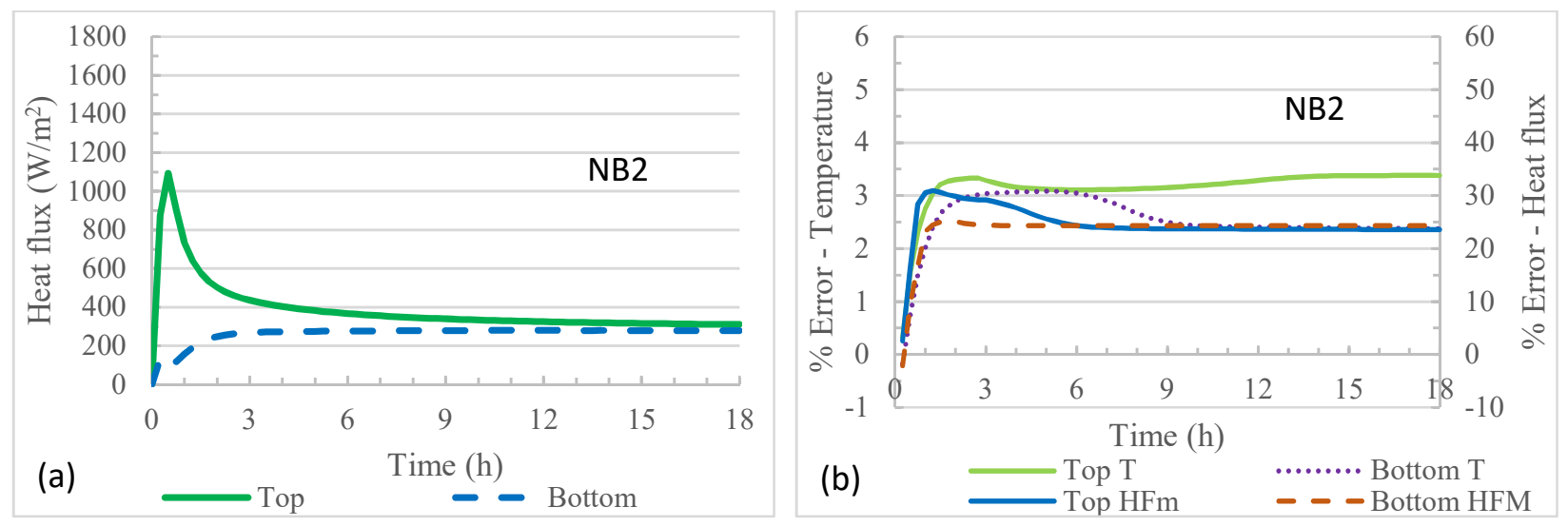

Figure 7.7. NB2 soil ( $\eta=0.56, S R=0.25)$ for the case of vertically heated from top: (a) measured heat fluxes and (b) \% errors in temperatures and heat fluxes.
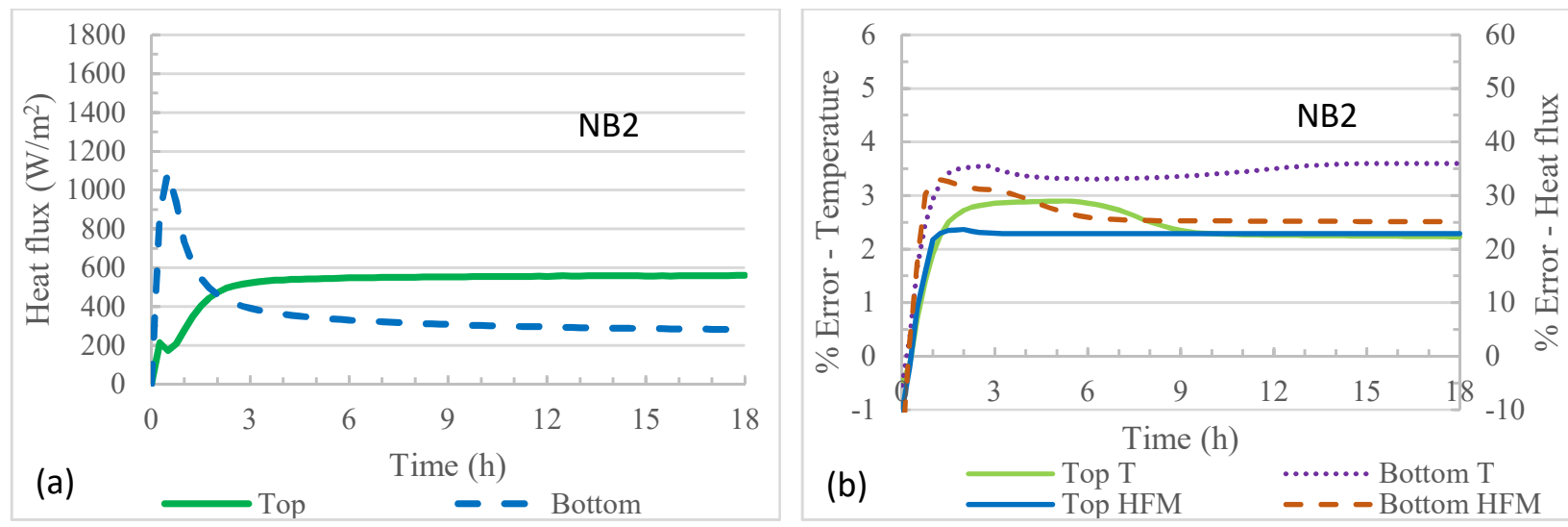

Figure 7.8. NB2 soil ( $\eta=0.53, S R=0.25)$ for the case of vertically heated from bottom: (a) measured heat fluxes and (b) \% errors in temperatures and heat fluxes. 


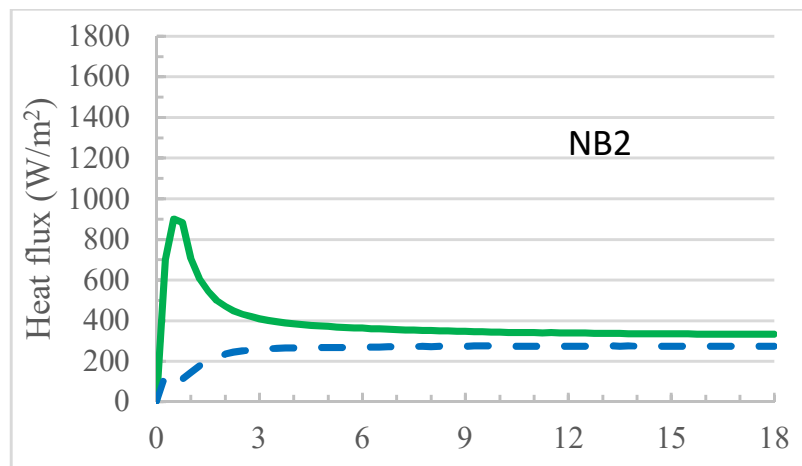

(a)
Time (h)

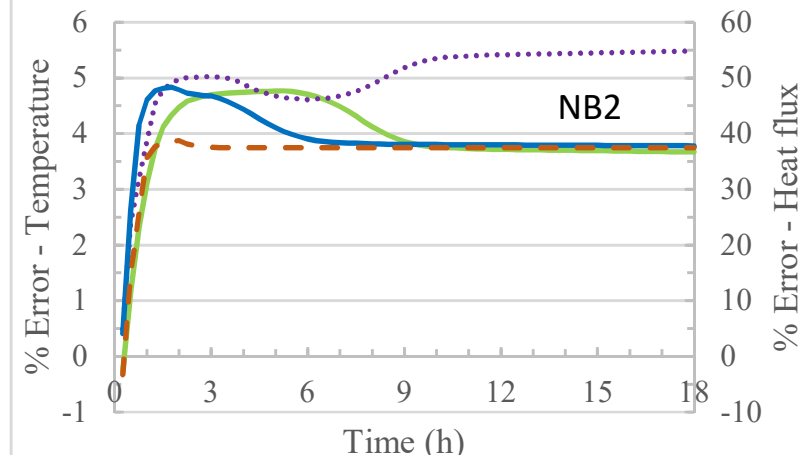

(b)
........ Bottom T

- - - Bottom HFM

Figure 7.9. NB2 soil ( $\eta=0.53, S R=0.25)$ for the case of horizontally heated: (a) measured heat fluxes and (b) \% errors in temperatures and heat fluxes.

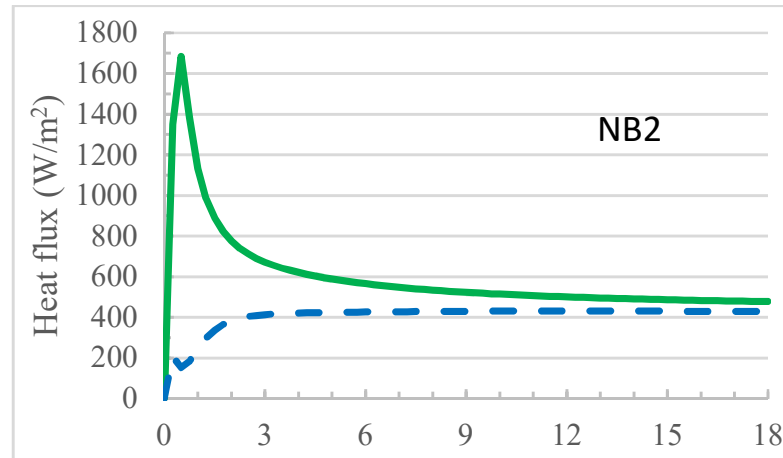

(a) $\longrightarrow$ Top
Time (h)

- Bottom

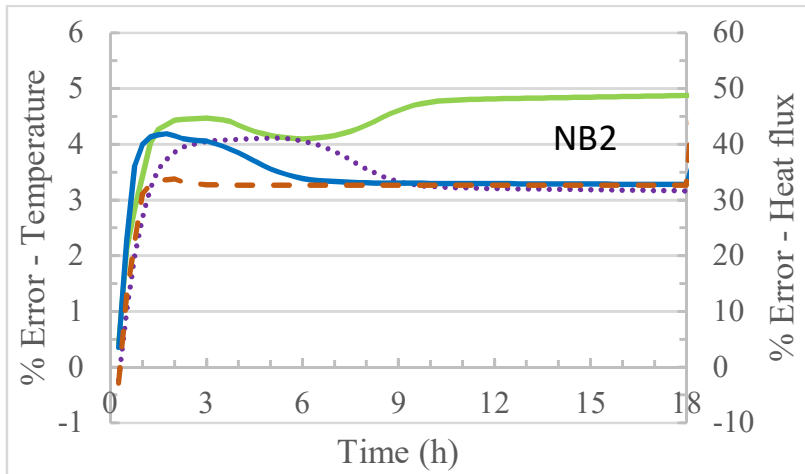

(b)
Time (h)

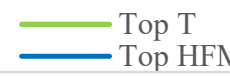

….... Bottom T
- - - Bottom HFm

Figure 7.10. NB2 soil ( $\eta=0.55, S R=0.50)$ for the case of vertically heated from top: (a) measured heat fluxes and (b) \% errors in temperatures and heat fluxes.

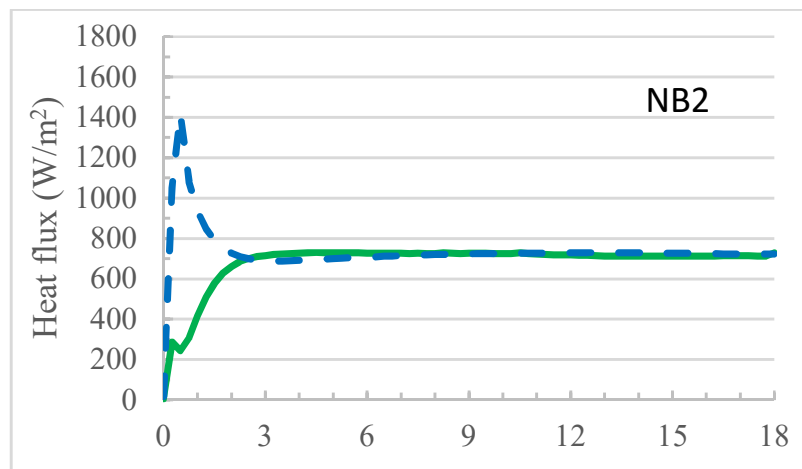

(a)

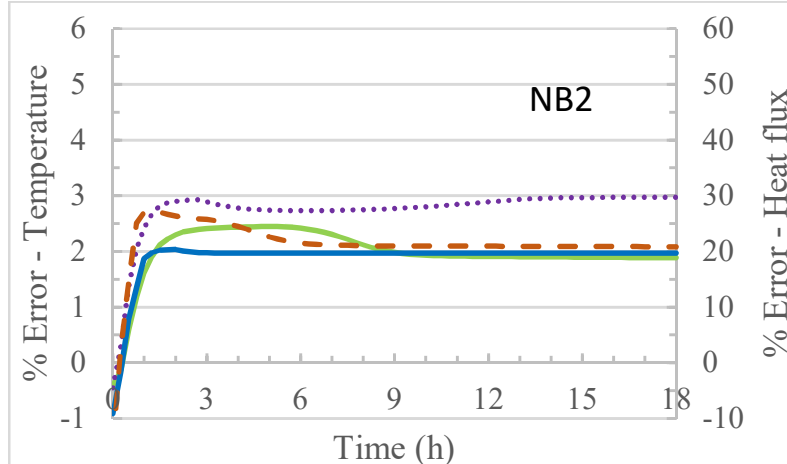

(b)
........ Bottom T - - - Bottom HFM

Figure 7.11. NB2 soil ( $\eta=0.53, S R=0.50)$ for the case of vertically heated from bottom: (a) measured heat fluxes and (b) \% errors in temperatures and heat fluxes. 

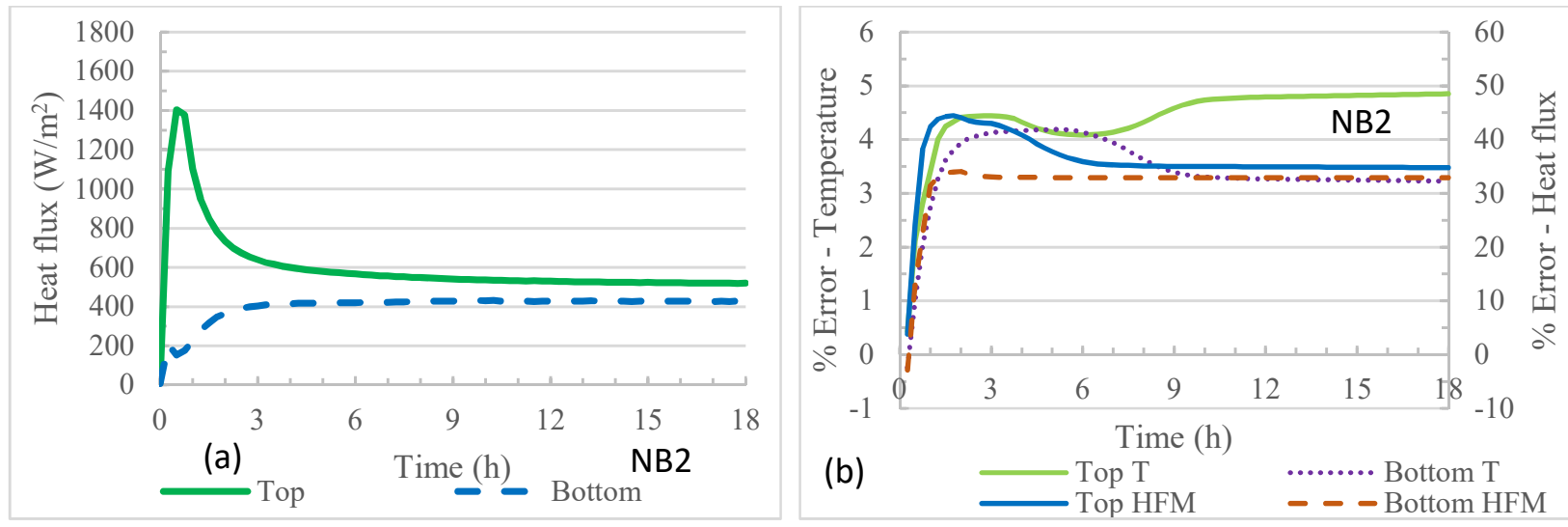

Figure 7.12. NB2 soil $(\eta=0.51, S R=0.50)$ for the case of horizontally heated: (a) measured heat fluxes and (b) \% errors in temperatures and heat fluxes.

\subsection{3 - QC2 Soil}

Figs. $7.13-7.18$ show the measured heat fluxes and comparisons of the PHCM simulations with the experimental values for wet QC2 soils under different testing conditions. The trend in each figure is similar to the corresponding trends for NB2 soil. Moreover, the heat flux at the hot side through QC2 soil is higher than through NB2 soil during transient time because QC2 is a coarser soil than NB2 is with $79.3 \%$ of sand content, while NB2 soil has no sand at all. Therefore, due to high quartz content, QC2 has higher thermal conductivity than NB2 has. For this reason, although the hot region of QC2 has lower moisture content than the hot region of NB2 has after a long time, QC2 still has higher heat transfer than NB2 has.
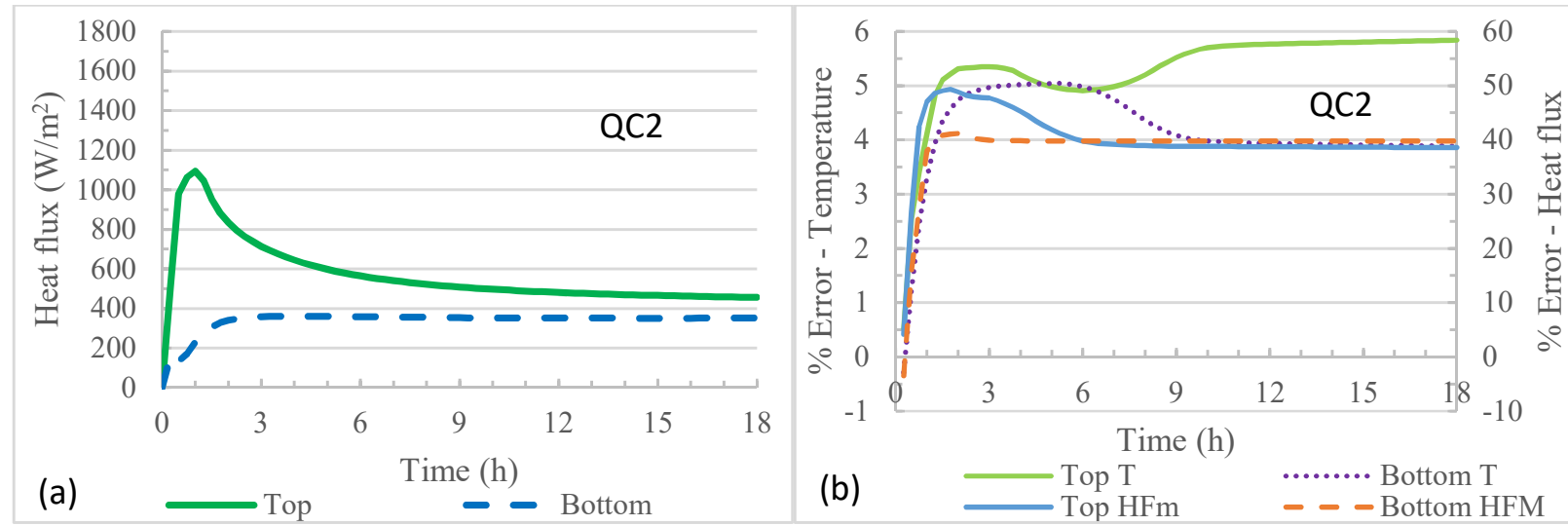

Figure 7.13. $Q C 2$ soil ( $\eta=0.50, S R=0.25)$ for the case of vertically heated from top: (a) measured heat fluxes and (b) \% errors in temperatures and heat fluxes. 


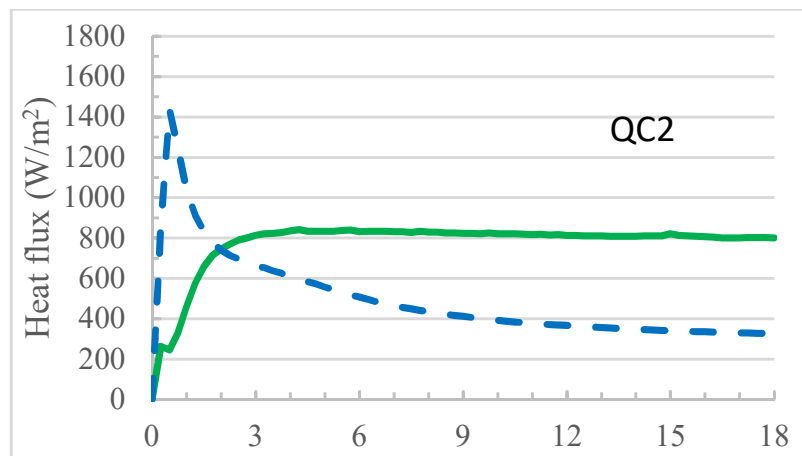

(a)
Time (h)

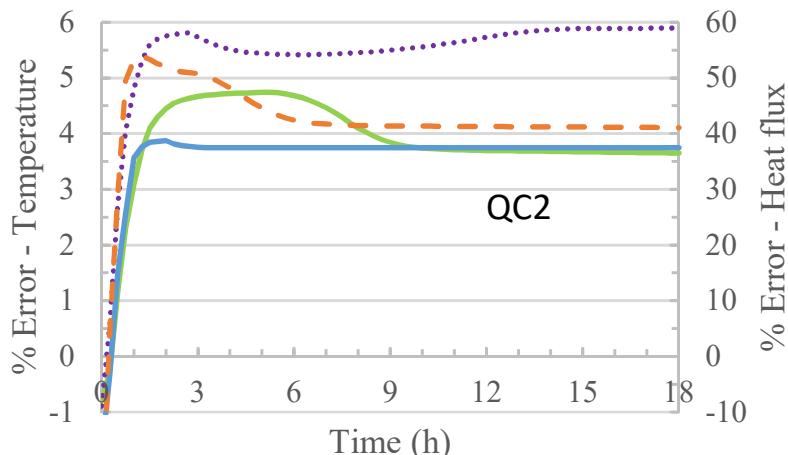

(b)
- Top T
Top HFM
........ Bottom T

- - - Bottom HFM

Figure 7.14. $Q C 2$ soil ( $\eta=0.48, S R=0.25)$ for the case of vertically heated from bottom: (a) measured heat fluxes and (b) \% errors in temperatures and heat fluxes.

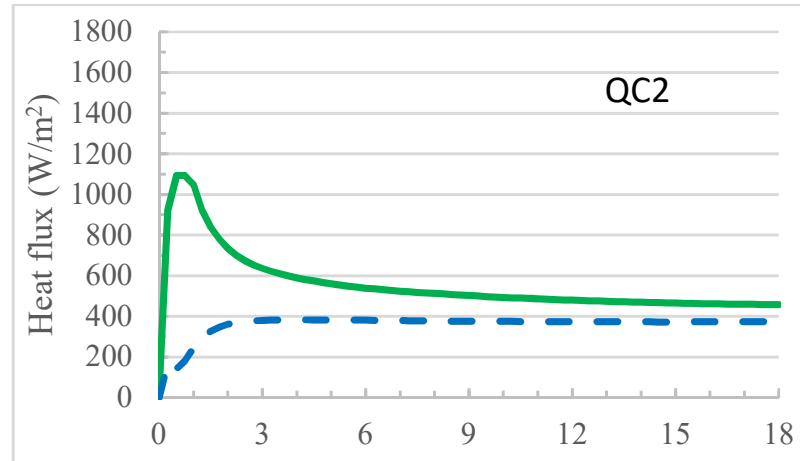

(a) Time (h) - Bottom

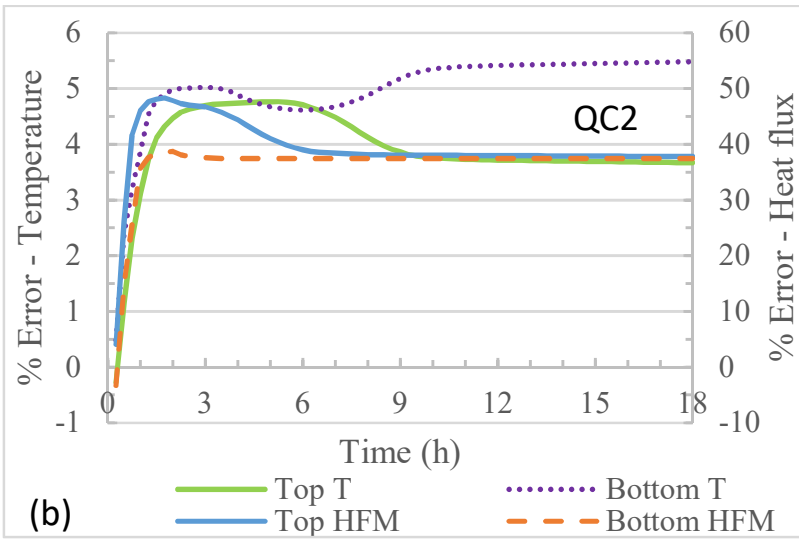

Figure 7.15. $Q C 2$ soil ( $\eta=0.47, S R=0.25)$ for the case of horizontally heated: (a) measured heat fluxes and (b) \% errors in temperatures and heat fluxes.

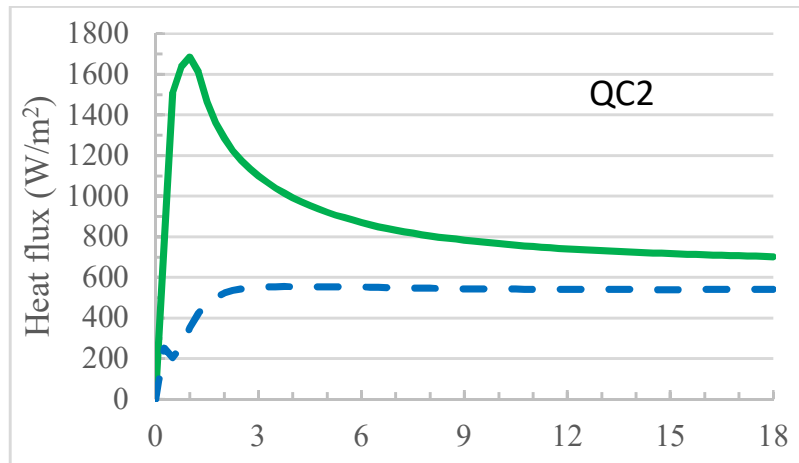

(a)
Time (h)

- Bottom

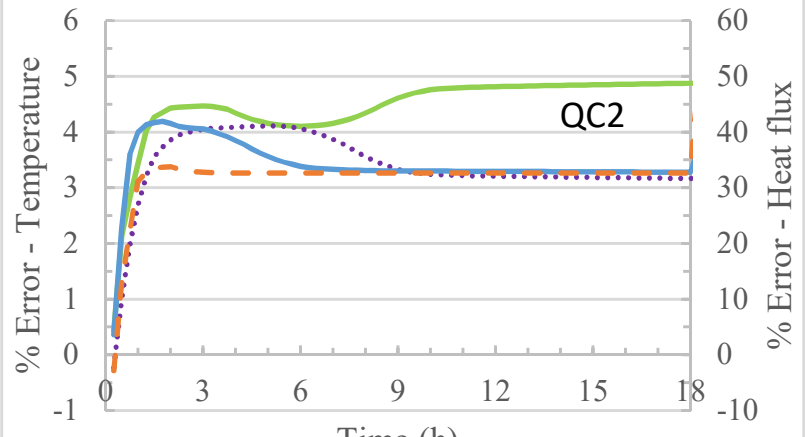

(b) Top T $\quad$ T....... Bottom T

Figure 7.16. $Q C 2$ soil $(\eta=0.44, S R=0.50)$ for the case of vertically heated from top: (a) measured heat fluxes and (b) \% errors in temperatures and heat fluxes. 

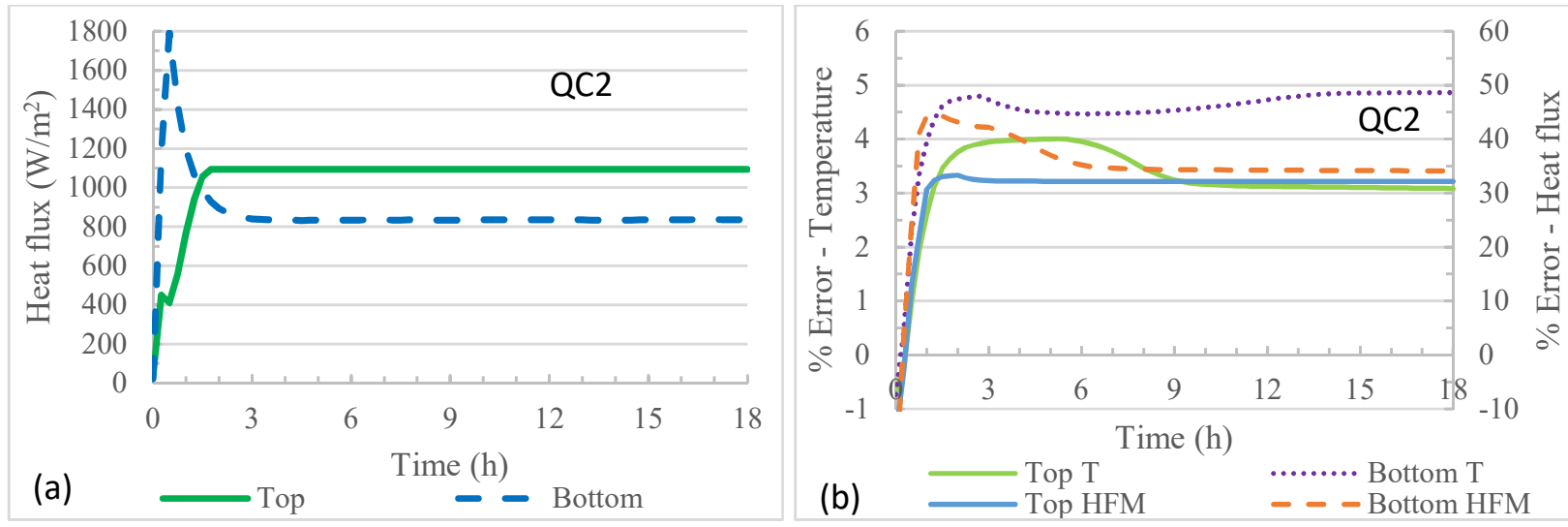

Figure 7.17. $Q C 2$ soil ( $\eta=0.44, S R=0.50)$ for the case of vertically heated from bottom: (a) measured heat fluxes and (b) \% errors in temperatures and heat fluxes.

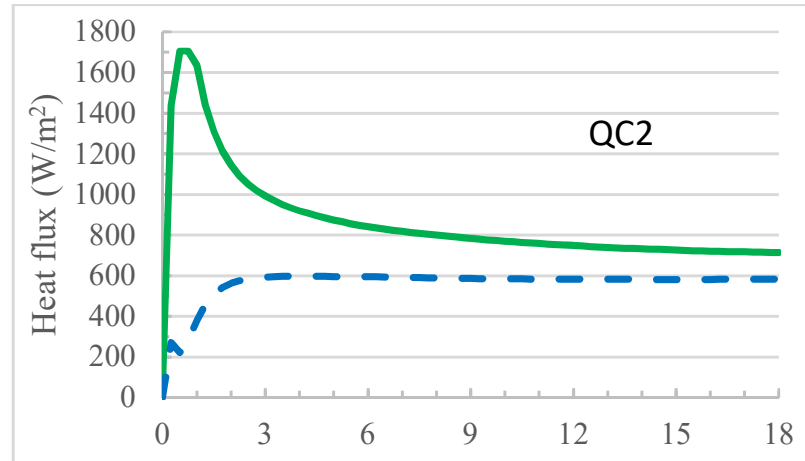

(a)

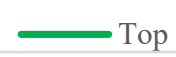

Time (h)

- Bottom

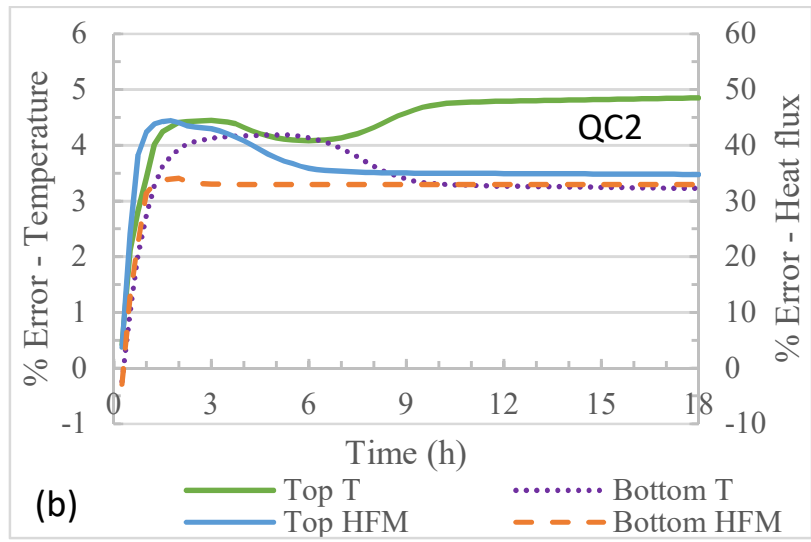

Figure 7.18. QC2 soil ( $\eta=0.42, S R=0.50)$ for the case of horizontally heated: (a) measured heat fluxes and (b) \% errors in temperatures and heat fluxes.

\section{2 - Moisture Transfer due to the Gravity}

\subsection{1-BC1 Soil}

The soil column was horizontally and gradually heated (with a maximum rate of $2{ }^{\circ} \mathrm{C}$ per hour) up to a temperature above $40^{\circ} \mathrm{C}$ during the experimentation. After reaching a desired temperature above $40^{\circ} \mathrm{C}$, the soil column was positioned vertically and kept at the temperature. As a result, isothermal condition could be assumed for the column after the column was positioned vertically and Eq. 2.5 was simplified to contain no thermal gradient. 
Fig. 7.19 shows the measured moisture contents of $\mathrm{BC} 1(\mathrm{SR} \approx 0.50)$ at $42^{\circ} \mathrm{C}$ and the comparison with the numerical solution of Eq. 2.5, i.e., from solving one-dimensional FVM formulations with 4437 finite volumes. The soil column is first positioned horizontally and gradually heated from room temperature up to $42^{\circ} \mathrm{C}$ by increasing the temperature settings of both water baths at a rate of $2^{\circ} \mathrm{C}$ per hour. When the soil column has achieved isothermal condition at $42^{\circ} \mathrm{C}$, the soil column is rotated to the vertical position to let the moisture move down due to the gravity. The $\%$ error is calculated as:

$$
\% \text { Error }=100 \% \times \frac{\theta_{\text {Eq.2.5 }}-\theta_{\text {experiment }}}{\theta_{\text {experiment }}}
$$

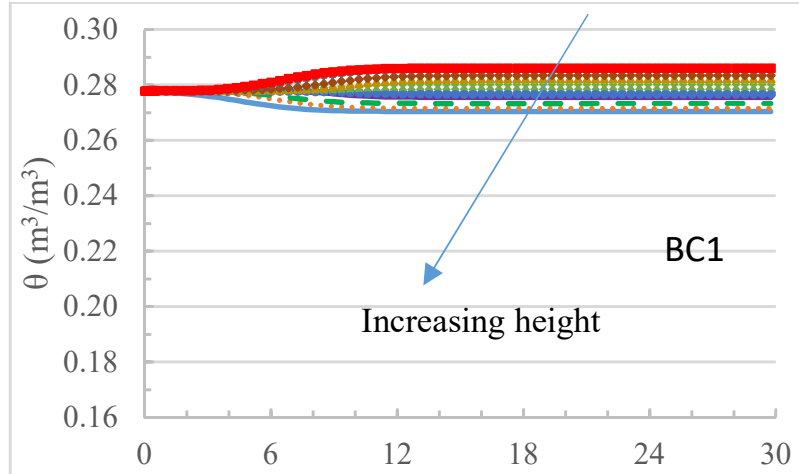

(a)

Time (h)

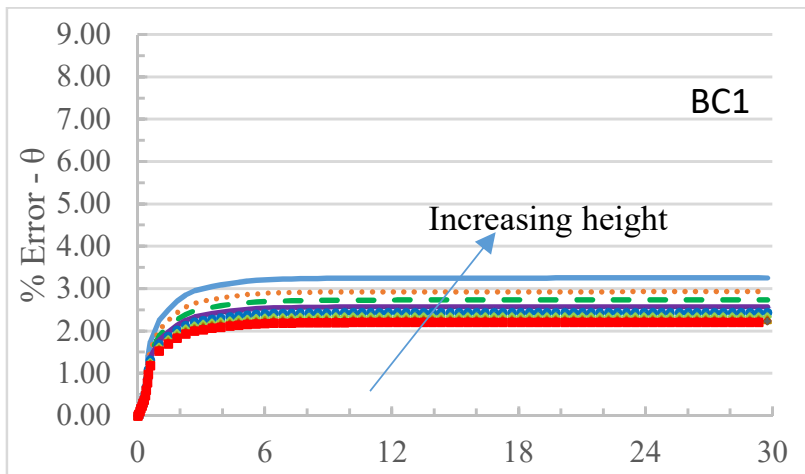

(b)

Time (h)

$\mathrm{N}$

$\mathrm{N} 2--\mathrm{N} 3 \longrightarrow \mathrm{N} 4$

$* \quad \mathrm{~N} 6 \quad \Delta \quad \mathrm{N} 7 \bullet \mathrm{N} 8+\mathrm{N} 9$

N10

Figure 7.19. (a) Measured moisture-content responses of $B C 1(\eta=0.56, S R \approx 0.50)$ at $42^{\circ} \mathrm{C}$ for the gravity test and (b) \% errors of simulated moisture contents.

As shown in Fig. 7.19a, due to higher elevations (N1 to N4), the gravity pulls the moisture down, so the moisture at the top moves down more than the ones below. In addition, with a higher temperature, the moisture (or water) is less viscous. As a result, the gravity can pull the moisture down faster as temperature increases. Moreover, because moisture at the bottom region cannot move down further, moisture accumulation from the top region over time is more at the bottom region. Consequently, the top region has more moisture movement than the bottom region has; hence the moisture movement predictions by Eq. 2.5 have higher errors at the higher elevations in the soil column as shown in Fig. 7.19b. The highest error of $3.25 \%$ for the moisture content is at 
the top (N1), which is acceptable because it is well within the experimental uncertainty of $14.5 \%$ (see Table 4.4).

Fig. 7.20 is based on similar experimental setup as Fig. 7.19, but the soil column is heated up to $52^{\circ} \mathrm{C}$. Because the temperature is higher, the moisture in the top moves down more and faster to the lower regions, due to lower viscosity and surface tension of the water. Consequently, the errors are higher and the maximum error at the top (N1) is about $3.71 \%$.
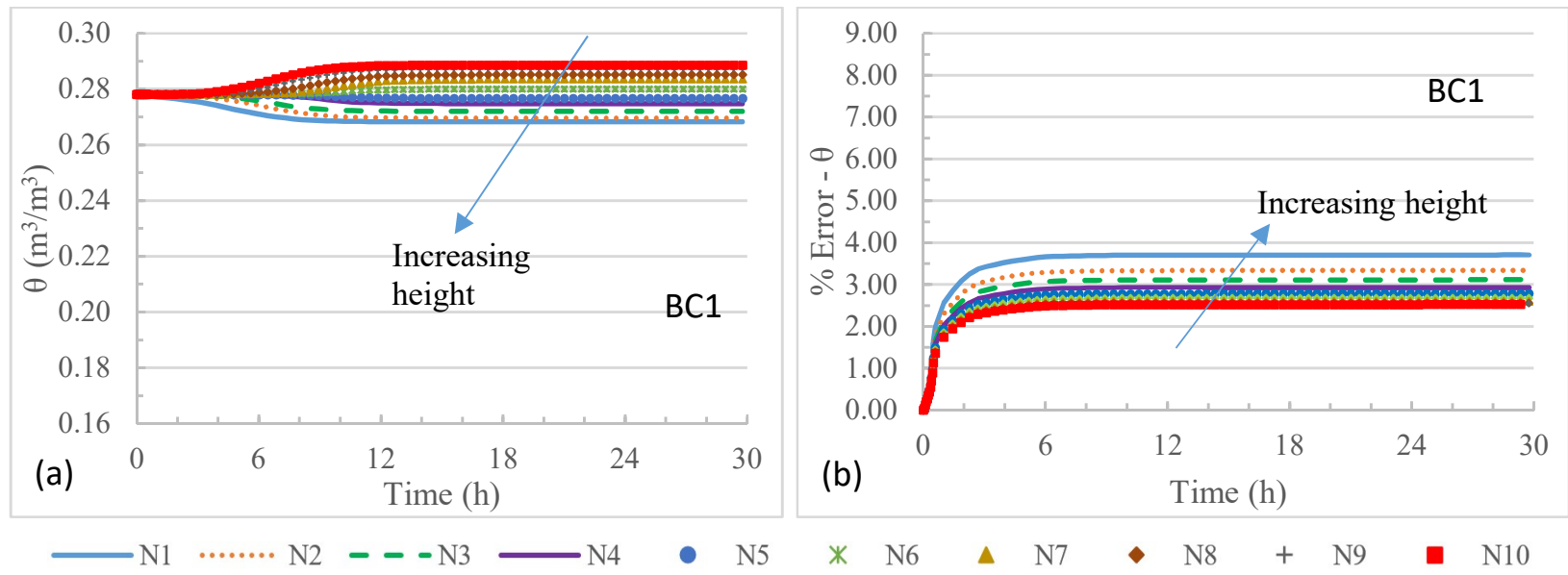

Figure 7.20. (a) Measured moisture-content responses of $B C 1(\eta=0.55, S R \approx 0.50)$ at $52^{\circ} \mathrm{C}$ for the gravity test and (b) \% errors of simulated moisture contents.

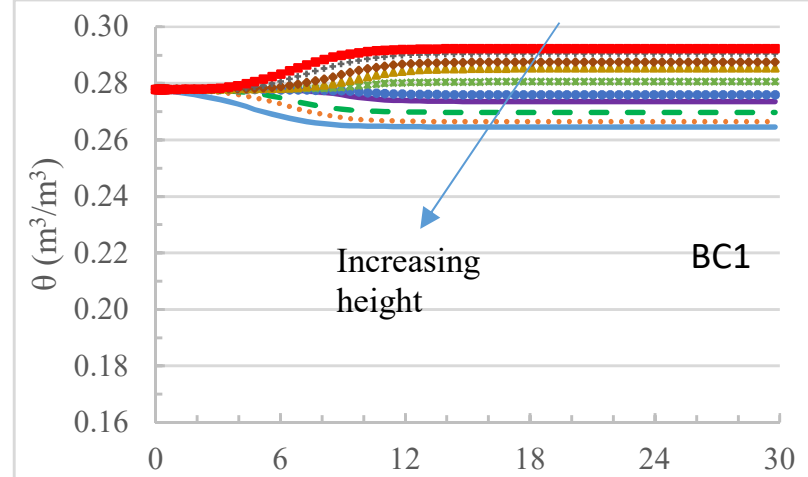

(a)

Time (h)

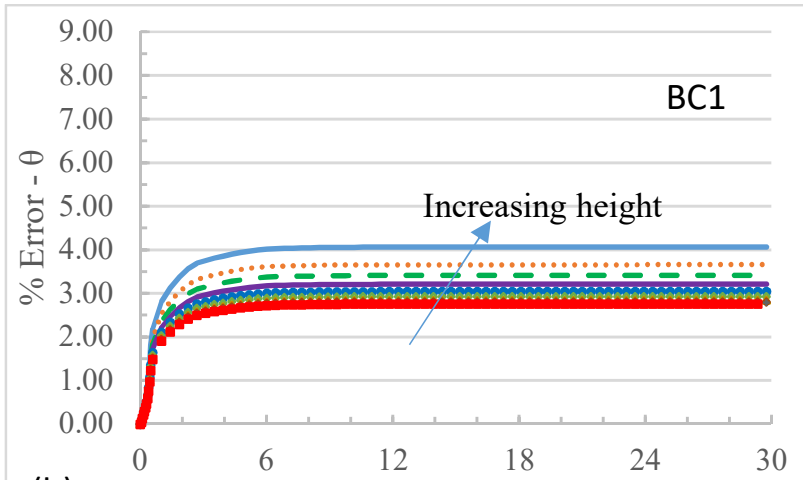

(b)
Time (h)

- $\mathrm{N} 8+\mathrm{N} 9$ - $\mathrm{N} 10$

Figure 7.21. (a) Measured moisture-content responses of $B C 1(\eta=0.55, S R \approx 0.50)$ at $62{ }^{\circ} \mathrm{C}$ for the gravity test and (b) \% errors of simulated moisture contents. 
Fig. 7.21 is based on similar experimental setup as Fig. 7.19, but the soil column is heated up to $62^{\circ} \mathrm{C}$. Similar to Fig. 7.20 , when the soil column is rotated vertically after being heated up to the desired higher temperature, the moisture (water in particular) is less viscous and can be pulled down more easily by the gravity. As a result, the top region loses more moisture while the bottom region gains more moisture. However, due to moisture accumulation in the bottom region, moisture is more restricted to flow downwards. Moreover, because the top region loses more water, the error is higher and the maximum error is about $4.06 \%$. From Figs. $7.19 \mathrm{~b}$ to $7.21 \mathrm{~b}$, it can be realized that the $\%$ errors of moisture contents are all positive values which indicate that the predicted moisture contents are all bigger than the measured ones. This can be due to the theoretical model (Eq. 2.5), the conversion from matric potential $\psi$ to volumetric moisture content $\theta$ by van Genuchten's model (Eq. A.10) or the combination of the two. However, the \% errors are well within the experimental uncertainty and are acceptable for engineering purposes.

\subsection{2-NB2 Soil}

Fig. 7.22 shows the measured moisture contents of $\mathrm{NB} 2(\mathrm{SR} \approx 0.50)$ at $42^{\circ} \mathrm{C}$ and the comparison with the numerical solution of Eq. 2.5. Similar to Fig. 7.19, the testing conditions for the soil column are the same, but the soil is NB2, instead of BC1. Because NB2 soil is coarser than $\mathrm{BC} 1$, the moisture moves down more and the predictions by Eq. 2.5 have higher errors.

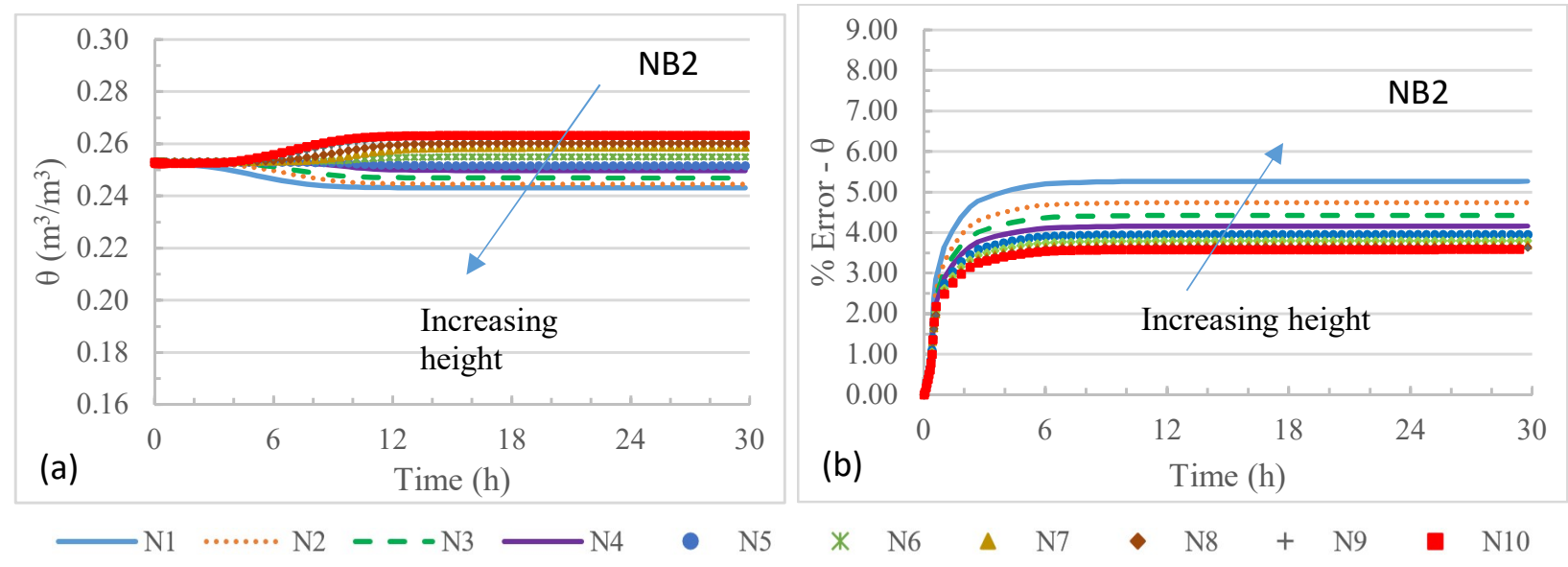

Figure 7.22. (a) Measured moisture-content responses of $N B 2(\eta=0.51, S R \approx 0.50)$ at $42{ }^{\circ} \mathrm{C}$ for the gravity test and (b) \% errors of simulated moisture contents. 
Figs. 7.23 and 7.24 are similar to Fig. 7.22, but the NB2 soil is uniformly heated to $52^{\circ} \mathrm{C}$ and $62^{\circ} \mathrm{C}$ respectively. The behaviors in Figs. 7.23 and 7.24 are similar to the corresponding figures for BC1. The highest errors in Figs. 7.23 and 7.24 are 5.82\% and 6.28\%, respectively, which are well within the experimental uncertainty and still acceptable. Similar to BC1 soil, the \% errors of moisture contents are positive values which indicate that the predicted moisture contents are bigger than the measured ones.

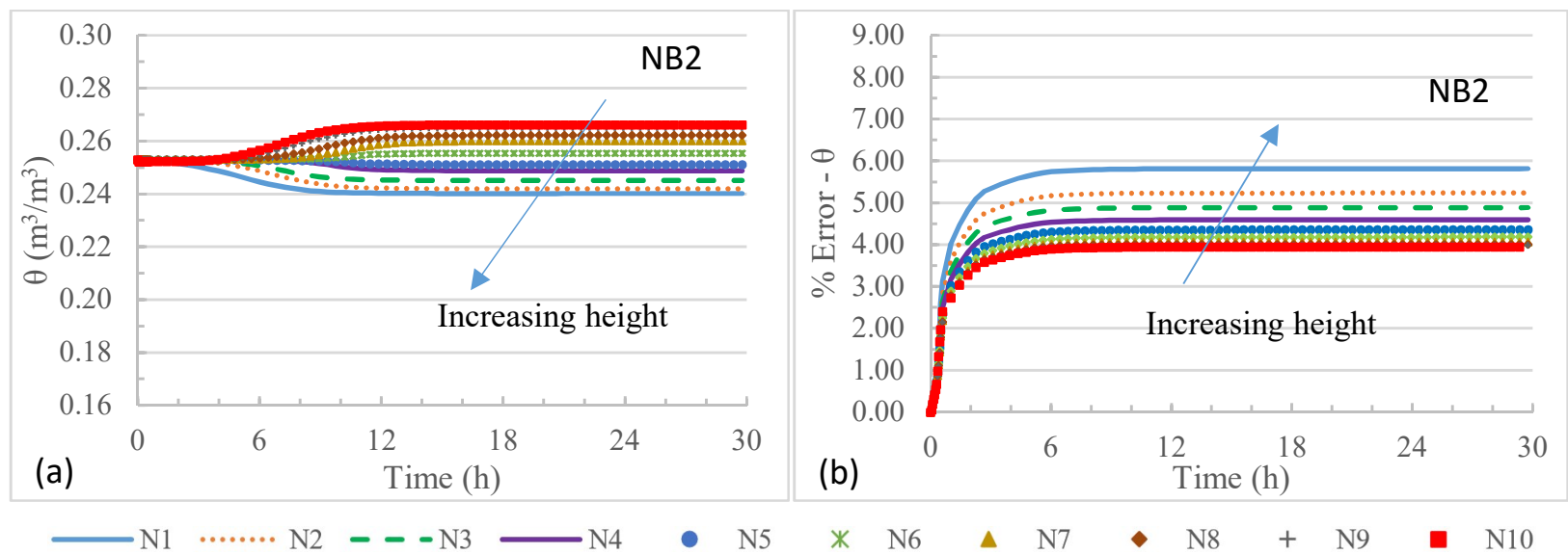

Figure 7.23. (a) Measured moisture-content responses of $N B 2(\eta=0.51, S R \approx 0.50)$ at $52^{\circ} \mathrm{C}$ for the gravity test and (b) \% errors of simulated moisture contents.

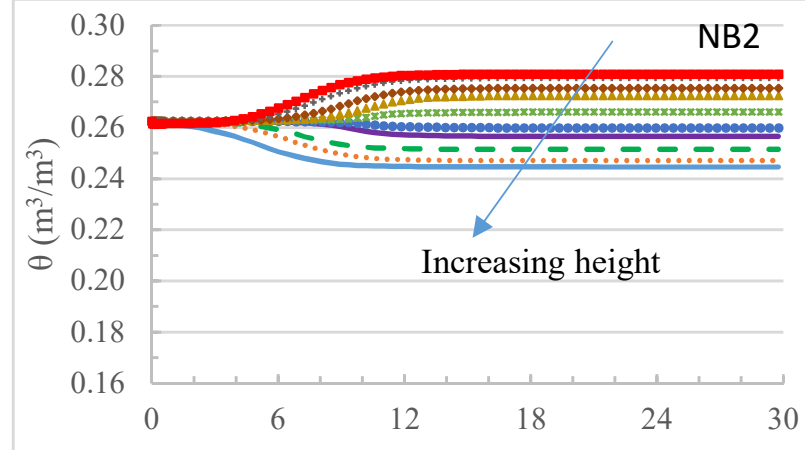

(a) Time (h)

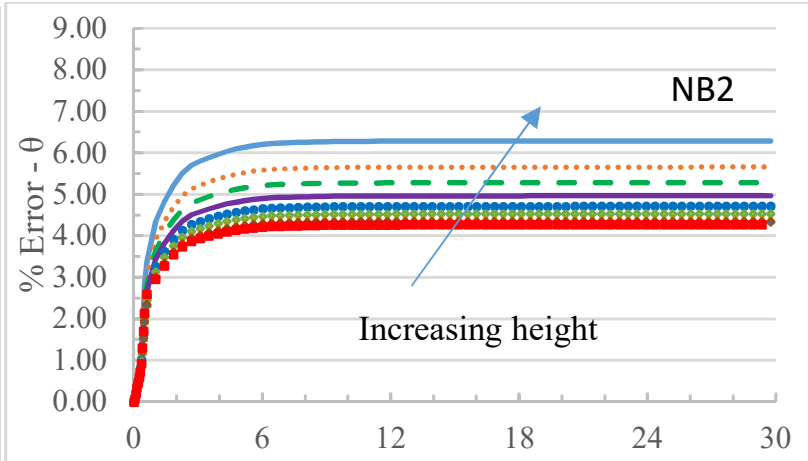

(b) Time (h)

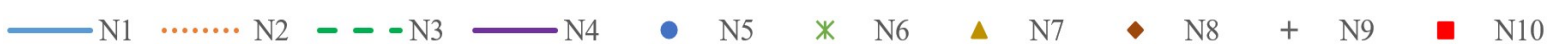

Figure 7.24. (a) Measured moisture-content responses of $N B 2(\eta=0.52, S R \approx 0.50)$ at $62{ }^{\circ} \mathrm{C}$ for the gravity test and (b) \% errors of simulated moisture contents.

\subsection{3-QC2 Soil}

Figs. $7.25-7.27$ show the measured moisture contents of QC2 $(\mathrm{SR} \approx 0.50)$ at $42^{\circ} \mathrm{C}$ and the comparison with the numerical solution of Eq. 2.5. Since QC2 is the coarsest soil, the moisture 
moves downwards the quickest and most among the three soils. As a result, the maximum errors for QC2 soils are the highest. In addition, unlike NB2 and BC1, from part (b) of Figs. 7.26 to 7.27, the $\%$ errors of moisture contents are negative values which indicate that the predicted moisture contents are smaller than the measured one in QC2 soil. This can be due to the theoretical model (Eq. 2.5), the conversion from matric potential $\psi$ to volumetric moisture content $\theta$ by van Genuchten's model (Eq. A.10) or the combination of the two. However, the maximum error of moisture content (i.e., $-8.02 \%$ in Fig. $7.27 b$ ) is well within the experimental uncertainty of $16.1 \%$ (see Table 4.4) and is still acceptable for engineering purposes.

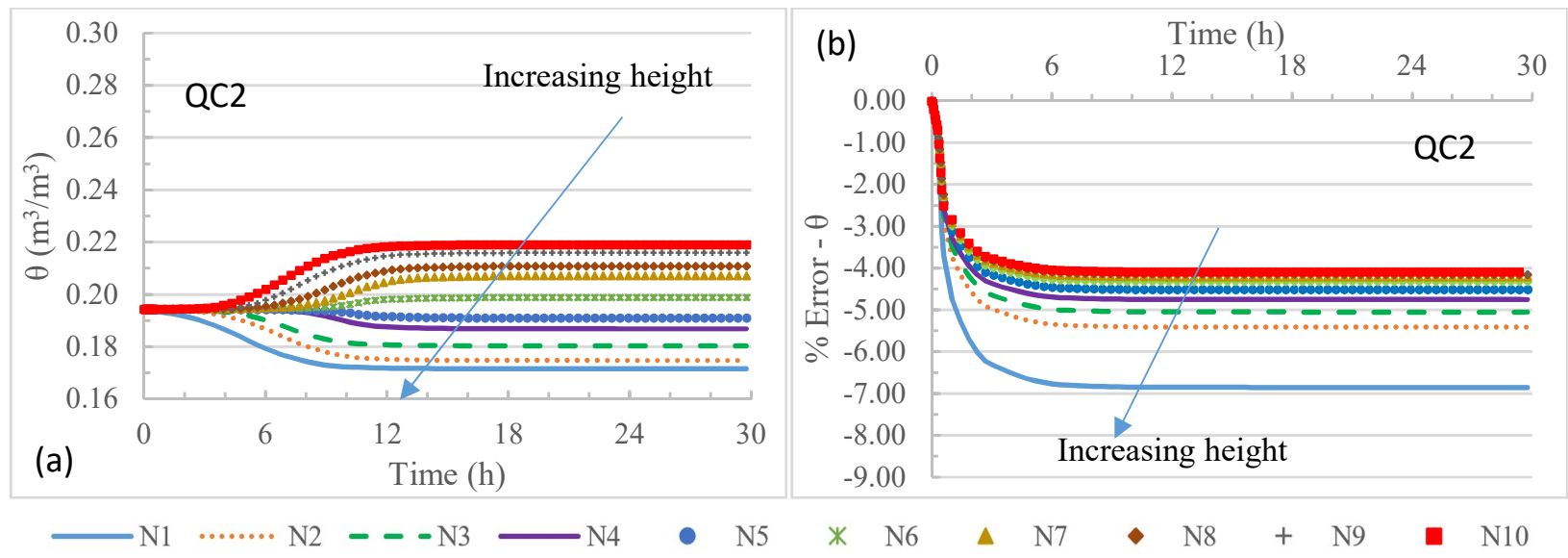

Figure 7.25. (a) Measured moisture-content responses of $Q C 2(\eta=0.45, S R \approx 0.50)$ at $42{ }^{\circ} \mathrm{C}$ for the gravity test and (b) \% errors of simulated moisture contents.

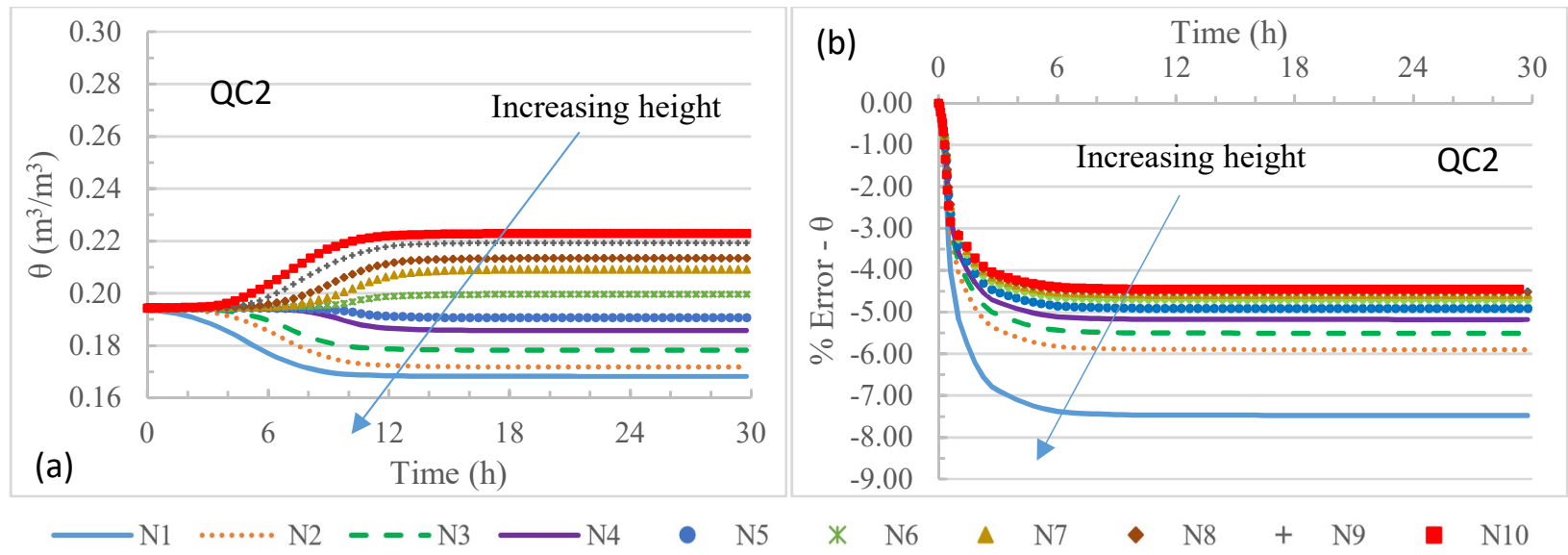

Figure 7.26. (a) Measured moisture-content responses of $Q C 2(\eta=0.42, S R \approx 0.50)$ at $52^{\circ} \mathrm{C}$ for the gravity test and (b) \% errors of simulated moisture contents. 


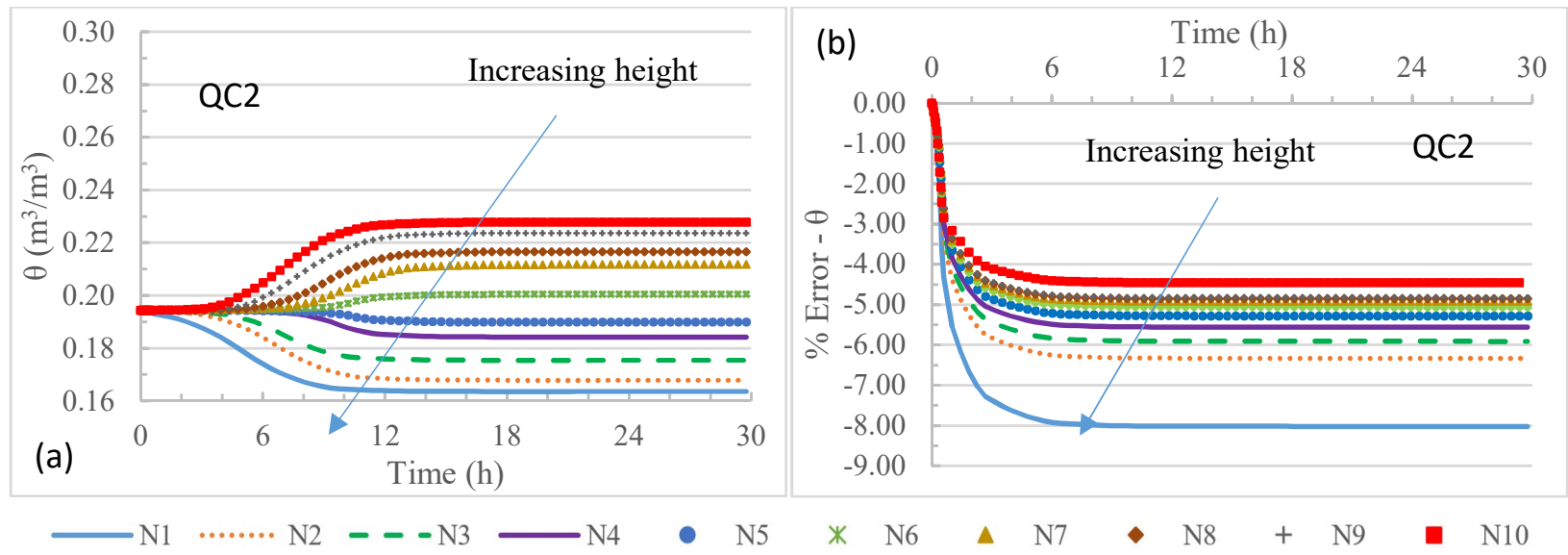

Figure 7.27. (a) Measured moisture-content responses of $Q C 2(\eta=0.42$, SR $\approx 0.50)$ at $62^{\circ} \mathrm{C}$ for the gravity test and (b) \% errors of simulated moisture contents.

\section{3 - Moisture Transfer due to Moisture Gradient}

\subsection{1 - Horizontal Position under Room Temperature}

The soil column initially has five layers of different saturation ratios (i.e., 0.00, 0.25, 0.40, 0.50 , and 0.70 ). During the soil preparation processes, the wetter layers were compacted earlier than the drier ones. After all five layers with different SRs were put into the soil column, the column was sealed, placed horizontally and tested at room temperature condition. The positions of the measuring needles are the same as in the previous cases. And Eq. 2.5 was simplified to contain no temperature and hydraulic conductivity gradients.

Fig. 7.28 shows the moisture-content responses of the $\mathrm{BC} 1$ soil column and compares the moisture-content responses with the numerical solution of Eq. 2.5. As shown in Fig. 7.28a, the moisture migration is noticeable due to moisture-content gradient, but it is not significant because $\mathrm{BC} 1$ is a fine soil with low permeability. From Fig. 7.28a, it can be seen that the moisture migration rate depends on the moisture-content gradient in the soil. For example, the moisture-content difference between the first layer $(\mathrm{SR} \approx 0.70)$ and the second layer $(\mathrm{SR} \approx 0.50)$ is $0.090 \mathrm{~m}^{3} / \mathrm{m}^{3}$, while the difference between the second layer and the third layer $(\mathrm{SR} \approx 0.40)$ is $0.044 \mathrm{~m}^{3} / \mathrm{m}^{3}$. Therefore, there is more moisture migrated from the first layer to the second layer than moisture migrated from the second layer to the third layer, resulting in a net gain of moisture in the second layer. Another interesting feature can also be observed from Fig. 7.28a. Although the moisture- 
content difference between the fourth layer $(\mathrm{SR} \approx 0.25)$ and the fifth layer $(\mathrm{SR} \approx 0.00)$ is big $(\Delta \theta$ $\approx 0.138 \mathrm{~m}^{3} / \mathrm{m}^{3}$ ), the moisture migration rate is actually quite slow in the dry layer. It takes more than 10 and 20 hours for the moisture finally reaches needles N2 and N1, respectively. The maximum error is about $4.54 \%$, which is still acceptable as compared to the experimental uncertainty of $18.2 \%$ (see Table 4.5 ).

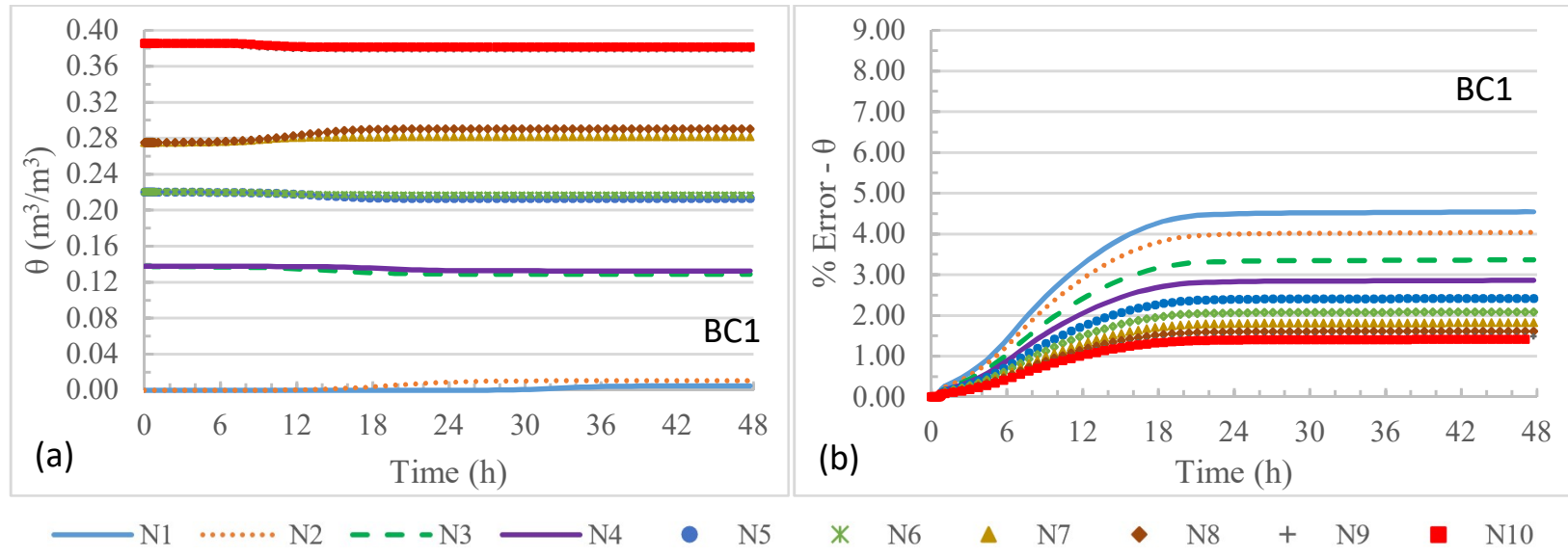

Figure 7.28. (a) Measured moisture-content responses of BC1 (in horizontal position) with five layers of different initial SRs and (b) \% errors of simulated moisture contents.

Fig. 7.29 has the same testing conditions as in Fig. 7.28, but the soil is NB2 instead of BC1. The results are very similar to BC1 with slightly more moisture movements. Because NB2 has lower moisture holding capacity and higher permeability, the moisture can migrate faster due to diffusion caused by moisture-content gradients. It seems that the theoretical model (Eq. 2.5) is less accurate in predicting higher moisture migration rates in a medium soil, especially in drier regions. The maximum error in predicting the moisture content in Fig. $7.29 \mathrm{~b}$ is about $5.91 \%$, which is still acceptable. 


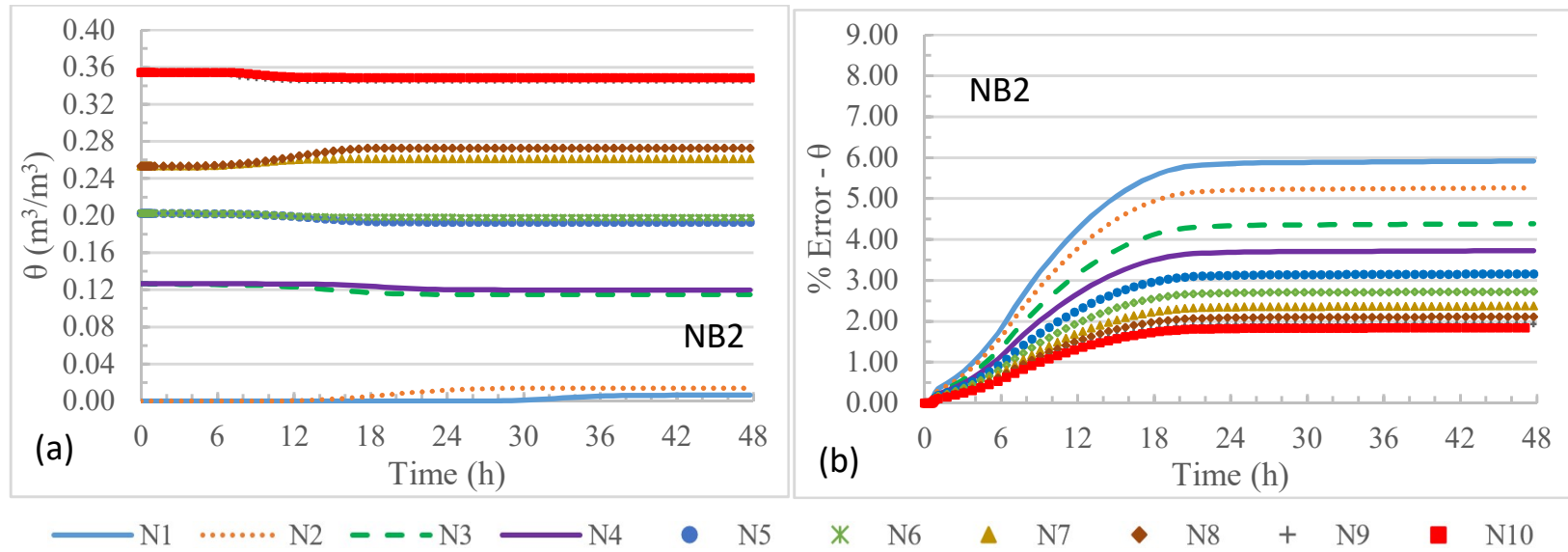

Figure 7.29. (a) Measured moisture-content responses of NB2 (in horizontal position) with five different initial SRs and (b) \% errors of simulated moisture contents.

Fig. 7.30 has the same testing conditions as in Figs. 7.28 and 7.29, but the soil is QC2 instead of BC1 and NB2. Similar to Figs. 7.28 and 7.29, the trend in Fig. 7.30a is very similar to those in Figs. 7.28a and 7.29a, but it has more moisture movements in the soil due to moisture-content gradients because QC2 is a coarse soil with the highest permeability among the tested three soils. But the magnitudes of errors are comparable to NB2 (Fig. 7.29b) with the maximum error of about $-5.85 \%$ which is still acceptable.

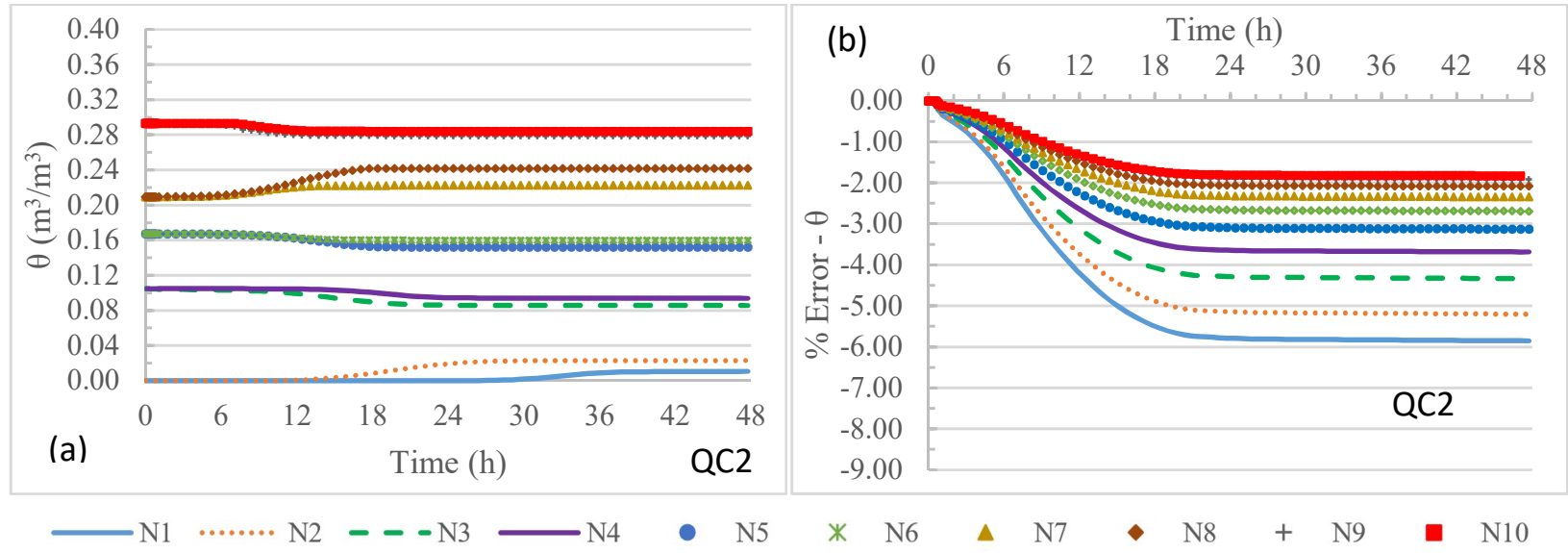

Figure 7.30. (a) Measured moisture-content responses of QC2 (in horizontal position) with five layers of different initial SRs and (b) \% errors of simulated moisture contents. 


\subsection{2 - Vertical Position under Room Temperature}

Similar to Section 7.3.1, the soil column is positioned vertically instead. Eq. 2.5 was simplified to contain no thermal gradient but the hydraulic gradient term still exists.

The case in Fig. 7.31 is similar to Fig. 7.28, but the $\mathrm{BC} 1$ soil column is positioned vertically with the wettest layer at the top at room temperature condition. Because the soil column is positioned vertically, the moisture movements are caused by moisture-content gradients and the gravity. Consequently, the moisture in Fig. 7.31 moves more quickly than in the case of Fig. 7.28, so the errors in moisture-content predictions are also higher with the maximum error at about $5.82 \%$ in the driest region, which is still acceptable as compared to the experimental uncertainties of up to $18.2 \%$ (see Table 4.5). It seems that the extra moisture migration due to the gravity has resulted in an additional maximum error of $1.28 \%$ in the driest region, as referenced to Fig. $7.28 \mathrm{~b}$. In the wettest region, only an additional error of $0.4 \%$ is observed due to the gravity.

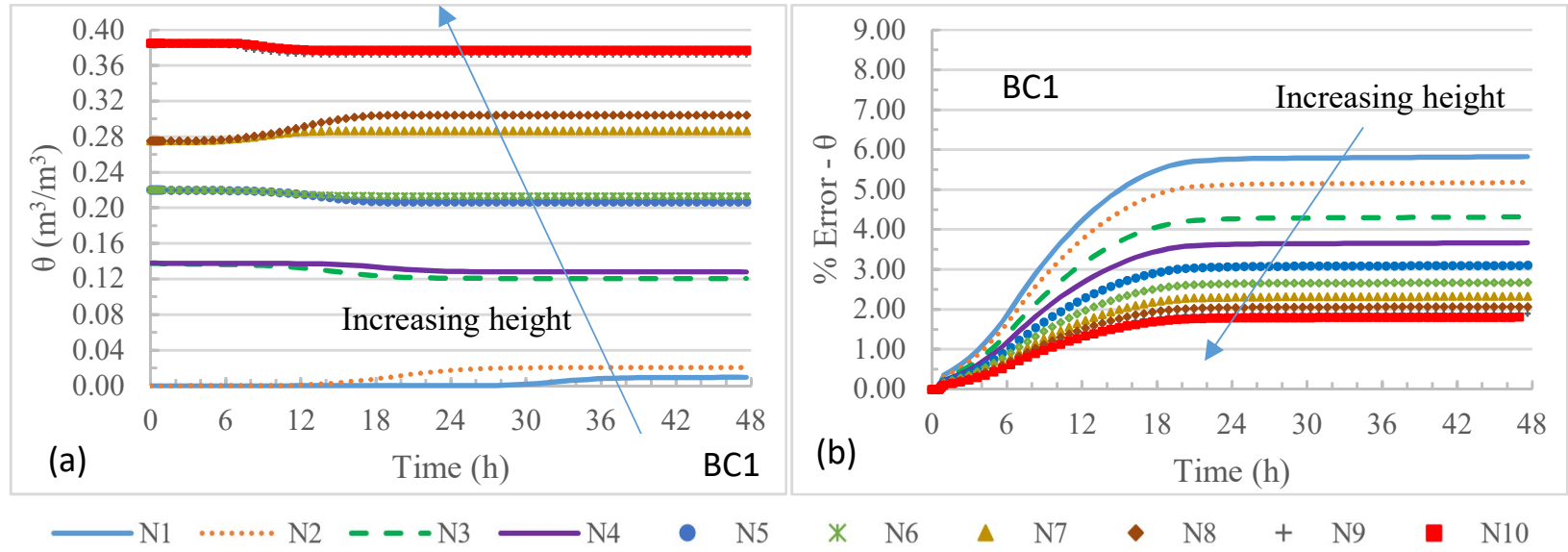

Figure 7.31. (a) Measured moisture-content responses of BCl (in vertical position) with five layers of different initial SRs and (b) \% errors of simulated moisture contents.

Fig. 7.32 has the same testing conditions as in Fig. 7.31 but the soil is NB2 instead of BC1. Similar to Fig. 7.31, the moisture from the wetter regions moves downwards due to moisturecontent gradients and the gravity, but it is slower than that in Fig. 7.22 because the moisture is more viscous at room temperature. The moisture-content responses are very similar to Fig. 7.31a; however, the errors of simulated moisture contents are larger. The maximum error is about $7.39 \%$ at needle N1 which is in the dry layer. For this case, the extra moisture migration due to the gravity 
has resulted in additional errors of $1.47 \%$ and $0.46 \%$ in the driest and wettest regions, respectively, as referenced to Fig. $7.29 \mathrm{~b}$.

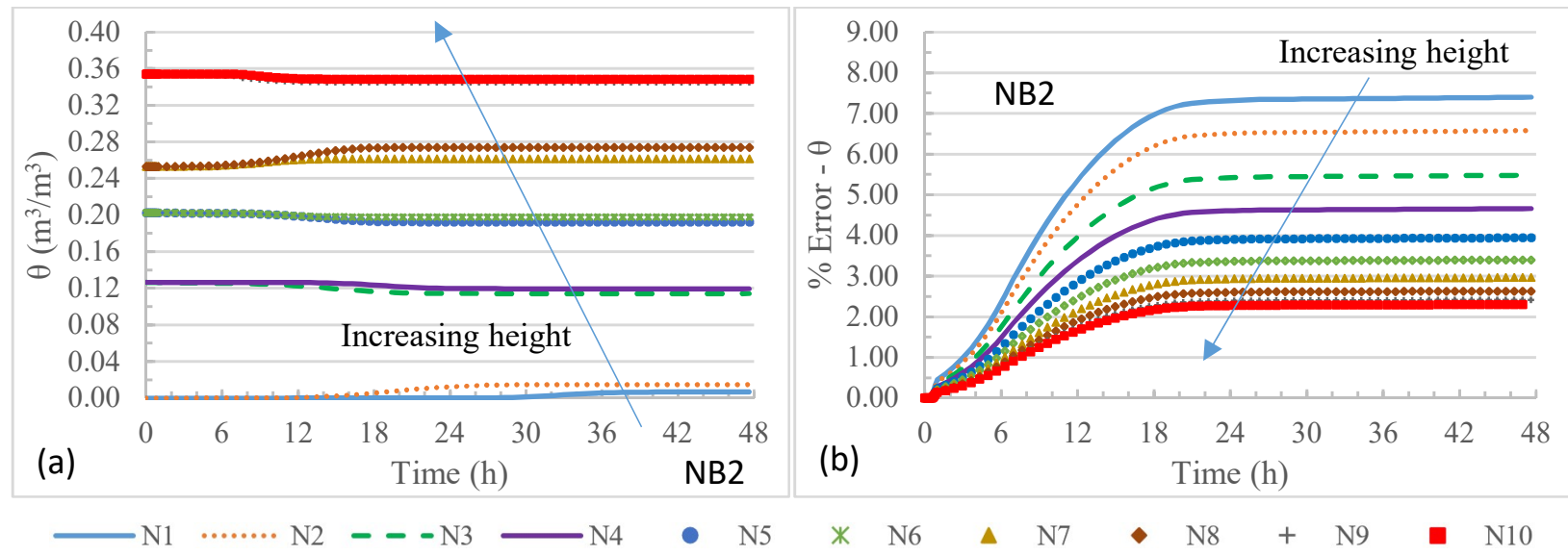

Figure 7.32. (a) Measured moisture-content responses of NB2 (in vertical position) with five different layers of initial SRs and (b) \% errors of simulated moisture contents.

Fig. 7.33 has the same testing conditions as in Figs. 7.31 and 7.32, but the soil is QC2 instead of BC1 and NB2. The trend in Fig. 7.33a is very similar to those in Figs. 7.31a and 7.32a. Because QC2 is the coarsest soil with the highest permeability among the three tested soil types, it cannot hold moisture (or water in particular) well. As a result, the moisture moves down quicker and the errors are also higher than those for $\mathrm{NB} 2$ and $\mathrm{BC} 1$ soils. But the magnitudes of errors are comparable to NB2 (Fig. 7.32b) with the maximum error of about $-7.80 \%$ which is still acceptable. 


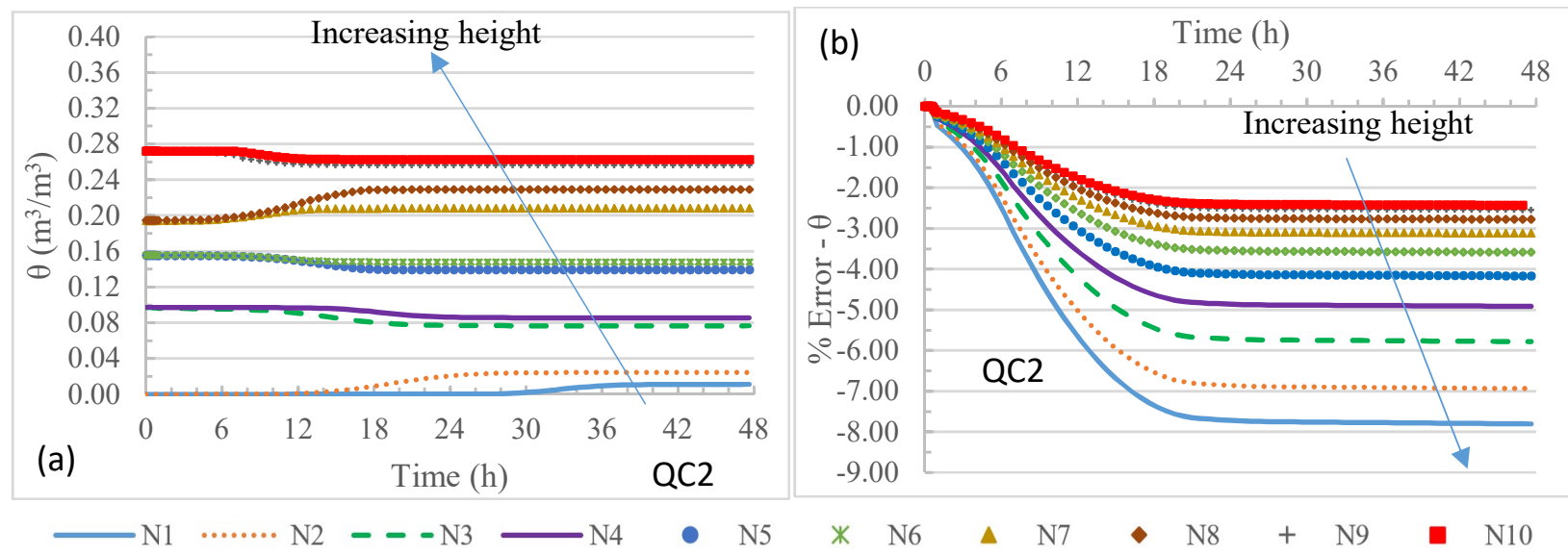

Figure 7.33. (a) Measured moisture-content responses of QC2 (in vertical position) with five layers of different initial SRs and (b) \% errors of simulated moisture contents.

\section{4 - Moisture and Thermal Transfers due to Combined Moisture and Thermal Gradients}

The soil column was experimented using differential heating (hot plate at $90^{\circ} \mathrm{C}$ and cold plate at $10^{\circ} \mathrm{C}$ ). Eq. 2.5 was used to numerically study the temperature and moisture responses in the soil column. The responses were coupled.

Fig. 7.34 shows the $\%$ errors (in terms of $T$ and $\theta$ ) of simulation results when the soil column contains $\mathrm{BC} 1$ soil $(\mathrm{SR} \approx 0.25)$ and is vertically heated from the top. The \% errors are calculated using Eq. 7.2. From the figure, it can be seen that the theoretical model (Eq. 2.5) over-predicts the moisture contents but under-predicts the temperatures. When the moisture content is overpredicted, the temperature becomes lower as there is more moisture (or water) around the region, resulting in higher thermal capacitance of the soil. Comparing Fig. 7.34a with Fig. 7.1b, it can be seen that the temperature predictions based on the coupled heat and moisture transfer equations (Eq. 2.5) at the top and bottom needles are more accurate than the pure conduction model (PHCM) using COMSOL. The reason is that the PHCM does not account for the moisture changes in the soil. Through the effects of the thermal gradient, the moisture near the hot plate moves and can significantly change the thermal conductivity of the region. However, the PHCM assumes that the thermal conductivity only varies with temperature. The maximum absolute errors of $T$ and $\theta$ are about $1.18 \%$ and $5.21 \%$ respectively. The errors are consistent with other experiments presented 
in Sections 7.2 and 7.3. It seems that whenever there is significant moisture migration, be it due to the gravity, thermal and/or moisture gradient, there will be about $1-2 \%$ and $4-6 \%$ errors for the temperature and moisture content, respectively. The simulations tend to over-predict moisture content and under-predict temperature of fine and medium soils. On the other hand, the opposite is true for coarse soils. It is not sure whether the theoretical model (Eq. 2.5), the conversion from matric potential $\psi$ to volumetric moisture content $\theta$ by van Genuchten's model (Eq. A.10) or the combination of the two is the cause of these discrepancies. However, these errors are acceptable for engineering applications.

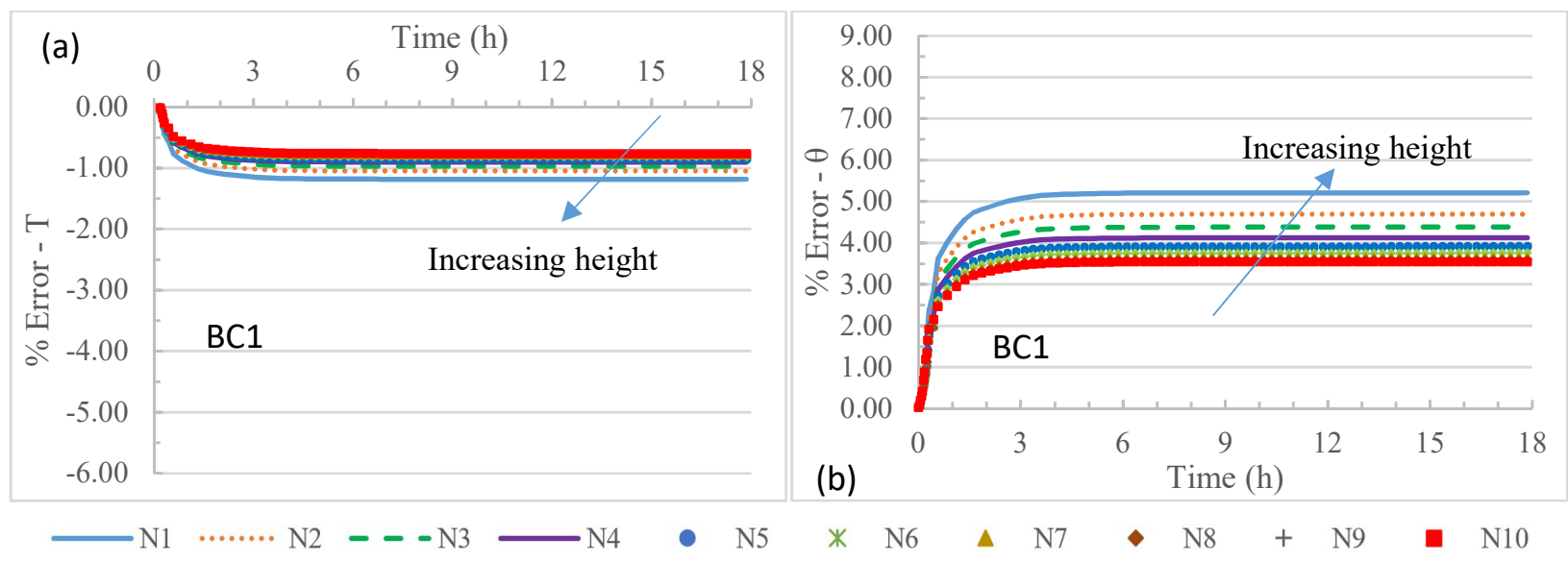

Figure 7.34. Percentage error of $T$ and $\theta$ (from Eq. 2.5) for BC1 soil (SR $\approx 0.25)$ and vertically heated from top.

Fig. 7.35 is based on the same experimental setup as Fig. 7.34, but the soil column is heated from the bottom, instead of the top. As it can be seen, the moisture contents are slightly more overpredicted than those in Fig. 7.34b. The maximum absolute errors of $T$ and $\theta$ are about $1.26 \%$ and $5.58 \%$ respectively. As the soil column is heated from the bottom, the hot air and water vapour can be buoyant up to transfer more heat and make the moisture less viscous, leading to faster moisture movement; hence the errors are higher. 


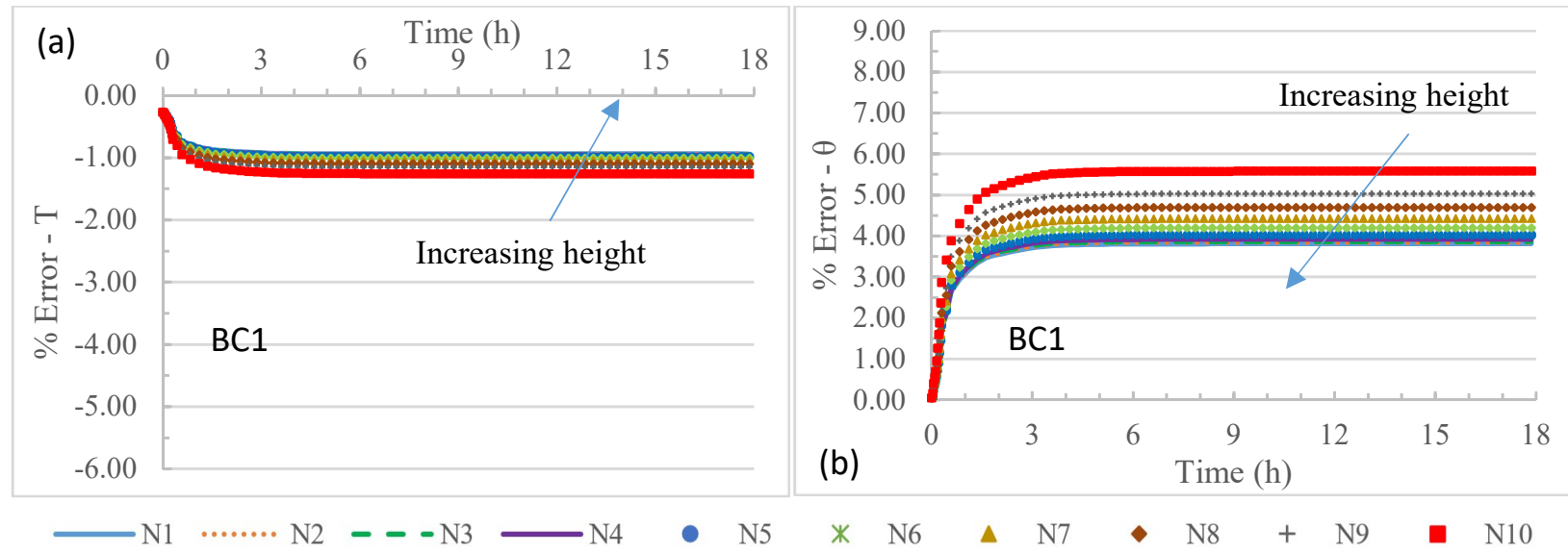

Figure 7.35. Percentage error of $T$ and $\theta$ (from Eq. 2.5) for $B C 1$ soil $(S R \approx 0.25)$ and vertically heated from bottom.

Fig. 7.36 is based on the same experimental setup as Fig. 7.34, but the soil column is horizontally heated, instead of being vertically heated from the top. The maximum absolute errors of $T$ and $\theta$ are about $1.04 \%$ and $5.08 \%$ respectively. Because the gravity is not acting in the same direction as the thermal gradient in horizontal heating, the moisture moves slower although the heat moves more (as shown in Chapter 6) and the moisture is less viscous than in Fig. 7.34.

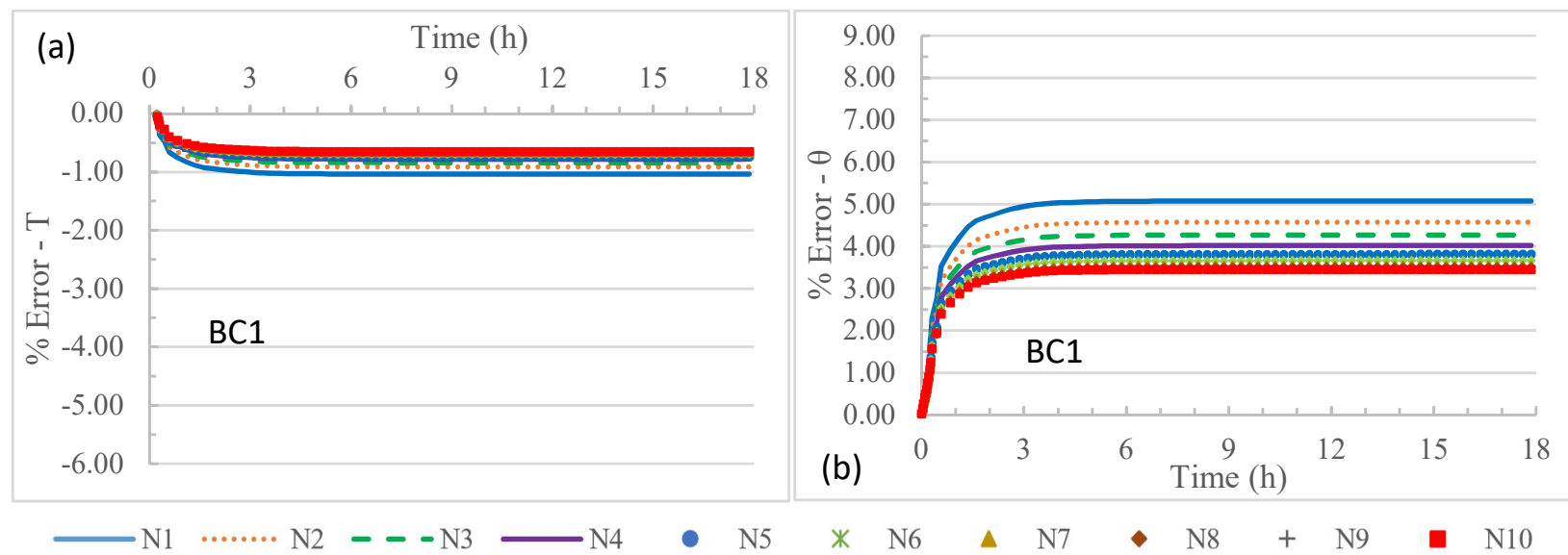

Figure 7.36. Percentage error of T and $\theta$ (from Eq. 2.5) for BC1 soil $(S R \approx 0.25)$ and horizontally heated.

Fig. 7.37 has the same testing conditions as in Fig. 7.34 but the $\mathrm{BC} 1$ soil is wetter ( $\mathrm{SR} \approx 0.50$ ). The maximum absolute errors of $T$ and $\theta$ are about $0.93 \%$ and $4.74 \%$ respectively. Because the soil contains more moisture, there is less void space for the moisture to move. Therefore, the errors are less. 


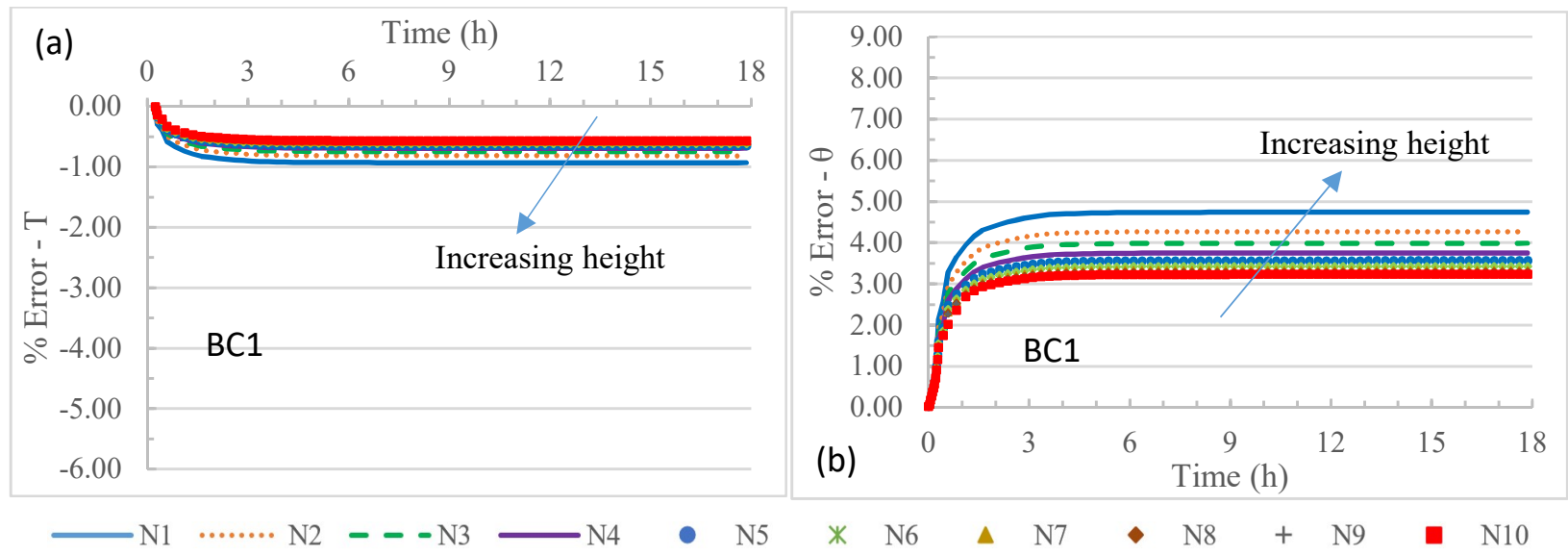

Figure 7.37. Percentage error of $T$ and $\theta$ (from Eq. 2.5) for $B C 1$ soil $(S R \approx 0.50)$ and vertically heated from top.

Fig. 7.38 is based on the same experimental setup as Fig. 7.37, but the soil column is heated from the bottom, instead of the top. The maximum absolute errors of $T$ and $\theta$ are about $1.00 \%$ and $4.86 \%$ respectively, which are very close to those in Fig. 7.37. However, compared with those in Fig. 7.35 (1.26\% and 5.58\%) of the same vertically heated from the bottom with $\mathrm{SR} \approx 0.25$, these errors are clearly smaller. This seems to indicate that when the moisture content is higher or less void space, the results will be more accurate. Obviously, the narrower void passages have more restriction to vapour migration, resulting in better results.

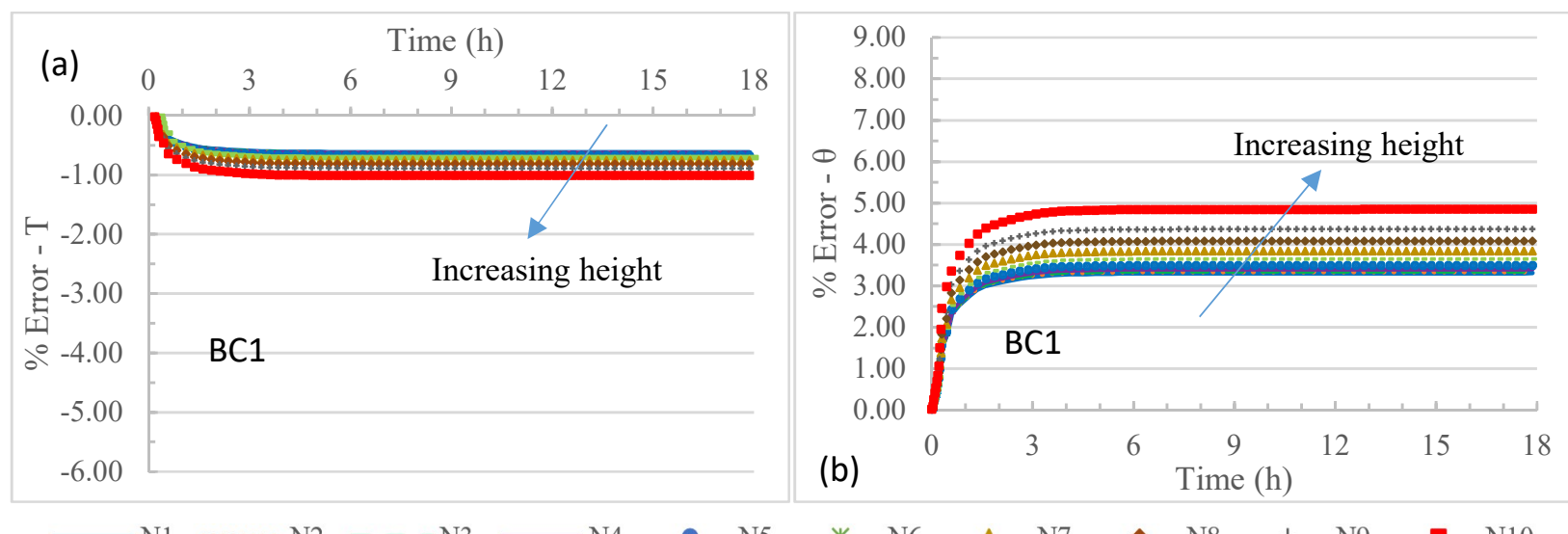

Figure 7.38. Percentage error of $T$ and $\theta$ (from Eq. 2.5) for $B C 1$ soil $(S R \approx 0.50)$ and vertically heated from bottom. 
Fig. 7.39 is based on the same experimental setup as Fig. 7.37, but the soil column is horizontally heated instead of being vertically heated from the top. The maximum absolute errors of $T$ and $\theta$ are about $0.89 \%$ and $4.67 \%$ respectively. Again, the errors are similar to Figs. 7.37 and 7.38. However, compared with those in Fig. 7.36 (1.04\% and 5.08\%) of the same horizontally heated with $\mathrm{SR} \approx 0.25$, these errors are again clearly smaller. So, the same conclusion as Fig. 7.38 above can be made that the results will be more accurate when the vapour migration is lower or the moisture content is higher.

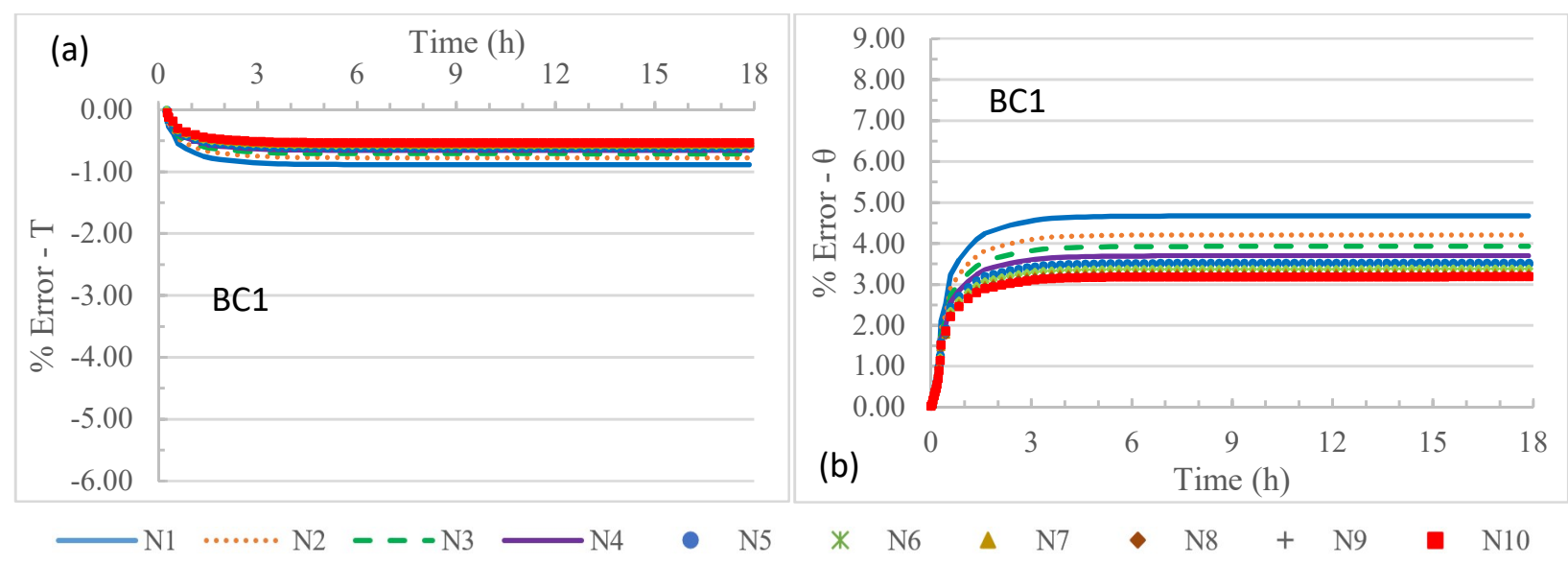

Figure 7.39. Percentage error of $T$ and $\theta$ (from Eq. 2.5) for BC1 soil (SR $\approx 0.50)$ and horizontally heated.

The cases in Figs. 7.34 - 7.39 are repeated for NB2 and QC2 soils with the same SRs and experimental conditions. Similarities are observed in testing BC1, NB2, and QC2 soils. As a result, Table 7.1 is made to summarize the worst \% errors (in terms of $T$ and $\theta$ ) of using Eq. 2.5.

From Table 7.1, it can be seen that Eq. 2.5 is less accurate in predicting the temperature responses and moisture movements in coarser soils. The reason is that coarser soils do not hold the moisture (or water) as well as finer soils do, so the moisture can flow through the coarser soils faster. Also, it can be seen that Eq. 2.5 is less accurate in predicting the temperature responses and moisture movements in drier soils. The reason is that drier soils have more void passages for vapour migration than wetter soils have, so the vapour moisture can flow through the soils faster. Consequently, Eq. 2.5 is less accurate in predicting the moisture movements and temperature responses in coarser and drier soils. 
The results in Table 7.1 are consistent with the previous results that simulations tend to overpredict moisture content and under-predict temperature of fine and medium soils and opposite for coarse soils. It is not sure whether the theoretical model (Eq. 2.5), the conversion from matric potential $\psi$ to volumetric moisture content $\theta$ by van Genuchten's model (Eq. A.10) or the combination of the two is the cause of these trends. These warrant a further investigation in order to pinpoint the cause in the future. However, in overall, the simulation results are well within the experimental uncertainties.

Table 7.1. Summary of worst \% errors (in terms of T and $\theta$ ) of using Eq. 2.5 to simulate different soils under various heating conditions.

\begin{tabular}{|c|c|c|c|c|c|c|c|c|c|c|c|}
\hline \multirow{2}{*}{\multicolumn{2}{|c|}{ Soil ID \& SR }} & \multicolumn{2}{|c|}{ Heating 1} & \multicolumn{2}{|c|}{ Heating 2} & \multicolumn{2}{|c|}{ Heating 3} & \multirow{8}{*}{ 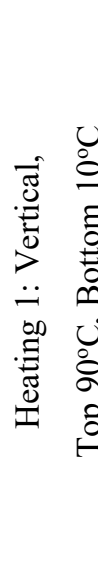 } & \multirow{8}{*}{\multicolumn{2}{|c|}{ 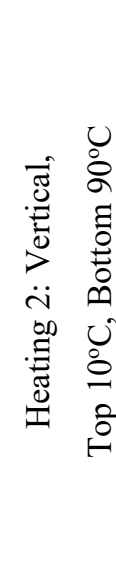 }} & \multirow[b]{8}{*}{$I$} \\
\hline & & $T$ & $\theta$ & $T$ & $\theta$ & $T$ & $\theta$ & & & & \\
\hline \multirow{2}{*}{$B C 1$} & 0.25 & -1.18 & +5.21 & -1.26 & +5.58 & -1.04 & +5.08 & & & & \\
\hline & 0.50 & -0.93 & +4.74 & -1.00 & +4.86 & -0.89 & +4.67 & & & & \\
\hline \multirow{2}{*}{$N B 2$} & 0.25 & -2.24 & +6.59 & -2.30 & +6.81 & -2.16 & +6.42 & & & & \\
\hline & 0.50 & -2.09 & +6.21 & -2.12 & +6.36 & -2.01 & +6.13 & & & & \\
\hline \multirow{2}{*}{$Q C 2$} & 0.25 & +3.45 & -7.44 & +3.52 & -7.53 & +3.39 & -7.31 & & & & \\
\hline & 0.50 & +3.31 & -7.12 & +3.35 & -7.20 & +3.28 & -7.04 & & & & \\
\hline
\end{tabular}

*Note: $90^{\circ} \mathrm{C}$ and $10^{\circ} \mathrm{C}$ are temperatures set at the water baths but are not the temperatures at the two boundaries (top and bottom or left and right) of the soil column.

\section{5 - Summary}

In this chapter, pure heat conduction model (PHCM) using COMSOL Multiphysics simulation package and the coupled heat and mass transfer model develop by Deru [28] were compared with experimental results. The experiments were set under various heating conditions for different soils (i.e., $\mathrm{BC} 1, \mathrm{NB} 2$, and $\mathrm{QC} 2$ ) with many different saturation ratios (from 0.00 to 0.70 ). The heating conditions included were:

- Vertical heating from the top and cooling at the bottom,

- Vertical heating from the bottom and cooling at the top,

- Horizontal heating on one side and cooling on the other side, 
- Vertical and uniform heating after horizontally gradual heating (less than $2^{\circ} \mathrm{C}$ per hour) on both sides of the soil column up to a high temperature (i.e., $42^{\circ} \mathrm{C}, 52^{\circ} \mathrm{C}$, or $62^{\circ} \mathrm{C}$ ), or

- Testing at room temperature $\left(\sim 22.5^{\circ} \mathrm{C}\right)$.

Due to measuring limitations, there were only 12 places in the soil column at where their temperatures could be recorded and two places at the top and bottom of the soil column at where the heat fluxes could be measured. Because the heat pulse method was used, periodic heat pulses were implemented to measure the thermal conductivities and heat capacities of the soil at the needles' locations. As shown in Section 7.1, the PHCM do not predict the moisture movements in soils. It over-predicts the temperature responses (about $2 \%$ for fine soil $\mathrm{BC} 1,3 \%$ for medium soil NB2, and 5\% for coarse soil QC2) and heat fluxes (15-40\%) in the soils. Also, it was noted that coarser soils could transfer more heat at the beginning of heating but transferred less heat after a long time of heating (more than 15 hours). The reason was coarser soils had more sand content which has higher thermal conductivity than clay or silk does. Because the soil was coarser, it could not hold moisture (or water) as well as the finer soils could. So after a long heating time, much of the moisture at the hot side had moved further away from the hot zone and the hot zone could become a better insulating layer.

The experimental moisture content was obtained by comparing the wet soil's volumetric heat capacity with the dry one using the following equation (see Appendix D for more details):

$$
\theta_{w} \approx \frac{C_{\text {wet soil }}-C_{d r y \text { soil }}}{\left(\rho C_{p}\right)_{w}}
$$

Because moisture in soils can take a long time to move in the pore space and the moving velocity can change depending on the temperature, experiments done under lower temperatures were usually run longer. The three time ranges were 18 hours, 30 hours, and 48 hours. Experimental results showed that coarser soils had higher moisture movements because coarser soils could not hold water as well as finer soils could. In addition, with the same soil, the moisture could move faster if the soil was put under higher temperatures. As the moisture moved faster, Eq. 2.5 by Deru [28] was less accurate in predicting the moisture movements in the soil.

Among vertical top heating, vertical bottom heating, and horizontal heating, Eq. 2.5 showed least errors in horizontal heating but highest errors in vertical bottom heating. The reason was from 
the gravity. With vertical bottom heating, the heat could be transferred more due to hot air and water vapour being buoyant up, which made the moisture less viscous and the gravity could pull the moisture down faster. Faster moisture velocity made Eq. 2.5 less accurate. In addition, it was noted that Eq. 2.5 showed less errors in predicting wetter soils of the same soil type (i.e., QC2, NB2, or BC1). The highest maximum absolute errors for predicting $T$ and $\theta$ (from the coarsest QC2 soils at $\mathrm{SR} \approx 0.25$ ) about 3.52\% and 7.53\% respectively. Because experimental uncertainties were more than the simulation errors, Eq. 2.5 was deemed sufficient in predicting the moisture movements and temperature responses in the soils. Moreover, in terms of engineering application, the simulations errors were deemed to be acceptable.

In the next chapter, conclusions for this thesis are made and future works are recommended. 


\section{CHAPTER 8 - CONCLUSIONS AND RECOMMENDATIONS}

\section{1 - Introduction}

Through literature survey, it was found that although theoretical models of heat and moisture transfer have been developed, there was no experiment performed under high temperatures (above $40^{\circ} \mathrm{C}$ ) to verify the models. As a result, this study was to verify one of the models by Deru [28]. An experimental soil cell made of stainless-steel tube with 63.5- $\mathrm{mm}$ inner diameter and 147.9- $\mathrm{mm}$ height was used for experimental studies of one-dimensional heat and moisture transfer within a vertical soil column. The soil cell was exposed to different heating conditions (i.e., isothermal heating, differential heating, and testing under room temperature) and different orientations (i.e., vertical and horizontal) to study the heat and moisture transfer in the soil column at different temperature levels and temperature differences. Five in-house-made heat pulse probes were inserted along the soil column in order to measure the soil's thermal conductivity, volumetric heat capacity, and moisture content.

The study includes four main parts:

1. Discretization of Deru's governing equations (i.e., Eq. 2.5) using one-dimensional and axisymmetric finite volume method (FVM) formulations. Matlab was used for the simulations.

2. Finite element simulations using COMSOL software package were made and found onedimensional FVM formulation was sufficient for this study.

3. Soils were put in the soil cell under different heating conditions and orientations. Experimental results (e.g., soil's thermal properties, temperature, and moisture content) were obtained. Uncertainties of the experiments were made to provide accuracy from the experiments.

4. Numerical verifications of Deru's theoretical model (i.e., Eq. 2.5) were made by comparing simulated results from Matlab with experimental data. It was found that Deru's governing equations provided good results. 


\section{2 - Research Contributions}

The major contributions of this research study are:

1. Development of a more accurate, reliable, and repetitive experimental methodology and apparatus to study heat and moisture transfer in soils from $40^{\circ} \mathrm{C}$ to $90^{\circ} \mathrm{C}$.

2. Development of one-dimensional and axisymmetric FVM formulations to verify for Deru's coupled heat and moisture transfer equations [28].

3. First comprehensive experimental study on the moisture transfer in three types of soil (fine, medium, and coarse textures) at room and high temperatures, different heating conditions, and various moisture flow scenarios for verifications of the theoretical model.

\section{3 - Concluding Remarks}

One-dimensional and axisymmetric FVM formulations were developed to numerically simulate the theoretical heat and moisture transfer in soils by Deru. Initial and boundary conditions were obtained from the experiments. The heat pulse method by Knight et al. [54] was used to calculate the thermal conductivity and volumetric heat capacity of the soils.

COMSOL software package was used to examine the applicability of one-dimensional simulation using the experimental soil column. It was found that the radial heat transfer was significantly lower than the axial one. The location of the temperature sensor in the actual needle of the probe is at about $0.01675 \mathrm{~m}$ of the arc length $(i . e ., r=1.675 \mathrm{~cm})$ and almost gives the average soil temperature at each cross section of the soil column. As a result, one-dimensional formulation is appropriate to numerically study the soil column.

Because of evaporation, the moisture of the wet soil in the beaker was lower than the moisture in the soil column. As a result, water was added more than required to the dry soil in the beaker to compensate for the losses due to evaporation. The overall porosity and actual moisture of the soil were calculated using Eqs. 3.1 - 3.3. 
The experiments were set under various heating conditions for different soils (i.e., BC1, NB2, and QC2) with many different saturation ratios (from 0.00 to 0.70 ). The heating conditions included were:

- Vertical heating from the top and cooling at the bottom,

- Vertical heating from the bottom and cooling at the top,

- Horizontal heating on one side and cooling on the other side,

- Vertical and uniform heating after gradual heating (maximum $2{ }^{\circ} \mathrm{C}$ per hour) at top and bottom of the soil column up to a high temperature (i.e., $42^{\circ} \mathrm{C}, 52^{\circ} \mathrm{C}$, or $62^{\circ} \mathrm{C}$ ), or

- $\quad$ Testing at room temperature (around $22.5^{\circ} \mathrm{C}$ ).

There were 12 places in the soil column at where their temperatures were recorded and two places at the top and bottom of the soil column at where the heat fluxes were measured. Because the heat pulse method was used, periodic heat pulses were implemented to measure the thermal conductivities and heat capacities of the soil at the needles' locations.

The pure heat conduction model (PHCM) using COMSOL was applied to compare with the experimental results. As shown in Section 7.1, the PHCM does not predict the moisture movements in soils. It over-predicts the temperature responses (about $2 \%$ for fine soil $\mathrm{BC} 1,3 \%$ for medium soil NB2, and 5\% for coarse soil QC2) in the soils and heat fluxes (15-40\%) at the top and bottom boundaries. Also, it was noted that coarser soils could transfer more heat at the beginning of heating but transferred less heat after a long time of heating (more than 15 hours). The reason was that coarser soils have more sand content which has higher thermal conductivity than clay or silk does. Because the soil is coarser, it cannot hold moisture (or water) as well as the finer soils can. So after a long heating time, much of the moisture at the hot side had moved further away from the hot zone and the hot zone could become a better insulating layer.

Due to the limited computing power, memory and time, the soil column cannot have a very large amount of nodes in the simulations. It is found from the grid sensitivity study that 4439 nodes (corresponding to 4437 control volumes with a grid size of $0.0333 \mathrm{~mm}$ each) is sufficient for the simulations of coupled heat and moisture transfer in the soil column.

Because moisture in soils can take a long time to move in the pore space and the velocity can change depending on the temperature, experiments done under lower temperatures were usually 
run longer. The three time ranges were 18 hours, 30 hours, and 48 hours. Experimental results showed that coarser soils had higher moisture movements because coarser soils could not hold water as well as finer soils could. In addition, with the same soil, the moisture could move faster if the soil was put under higher temperatures. As the moisture moved faster, Eq. 2.5 by Deru [28] was less accurate in predicting the moisture movements in the soil.

Among vertical top heating, vertical bottom heating, and horizontal heating, Eq. 2.5 showed least errors in horizontal heating but highest errors in vertical bottom heating. The reason was from the gravity. With vertical bottom heating, the heat could be transferred more due to hot air and water vapour being buoyant up, which made the moisture less viscous and the gravity could pull the moisture down faster. Faster moisture velocity made Eq. 2.5 less accurate. In addition, it was noted that Eq. 2.5 showed less errors in predicting wetter soils of the same soil type (i.e., QC2, $\mathrm{NB} 2$, or $\mathrm{BC} 1$ ). The highest maximum absolute errors for predicting $T$ and $\theta$ (from the coarsest QC2 soils at $\mathrm{SR} \approx 0.25$ ) are about $3.52 \%$ and $7.53 \%$ respectively. Because experimental uncertainties were more than the simulation errors, Eq. 2.5 was deemed sufficient in predicting the moisture movements and temperature responses in the soils. Moreover, in terms of engineering applications, the simulations errors were deemed to be acceptable.

\section{4-Recommendations for Future Work}

Although soils from coarse to fine textures were tested, there are still rooms for further improvements and studies as follow:

1. Explore other soils to understand how their thermal properties change and how Deru's governing equations (i.e., Eq. 2.5) perform on the soils.

2. Run the water baths at different temperatures so that the soil column can be exposed to different temperature levels and differences in order to obtain more comprehensive data for more verification of Eq. 2.5.

3. Work on the axisymmetric (instead of one-dimensional) formulations of Eq. 2.5 and compare the differences. 


\section{APPENDICES}

\section{A-Material Properties}

A.1 - Properties for Air and Water [28]

$$
\begin{gathered}
k_{a}=0.02417+7.596 \times 10^{-5}(T-273.15) \quad \mathrm{W} / \mathrm{m} \cdot \mathrm{K} \\
k_{w}=0.5694+1.847 \times 10^{-3}(T-273.15)-7.394 \times 10^{-6}(T-273.15)^{2} \quad \mathrm{~W} / \mathrm{m} \cdot \mathrm{K} \\
\rho_{w}=1000-4.77 \times 10^{-2}(T-273.15)-3.8 \times 10^{-3}(T-273.15)^{2} \quad \mathrm{~kg} / \mathrm{m}^{3} \\
\sigma_{w a}=0.1171-1.516 \times 10^{-4} T \mathrm{~N} / \mathrm{m}
\end{gathered}
$$

where $T$ is in $\mathrm{K}$.

Kinematic viscosity of water:

$$
\begin{aligned}
v_{w}= & 1.71806 \times 10^{-6}-4.46108 \times 10^{-8}(T-273.15)+5.36977 \times 10^{-10}(T-273.15)^{2} \quad \mathrm{~m}^{2} / \mathrm{s} \\
& -2.35693 \times 10^{-12}(T-273.15)^{3}
\end{aligned}
$$

where $T$ is in $\mathrm{K}$.

Water's latent heats of fusion and vaporization:

$$
\begin{gathered}
L_{f}=3.338 \times 10^{5} \mathrm{~J} / \mathrm{kg} \\
h_{f g}=2.501 \times 10^{6}-2405(T-273.15) \mathrm{J} / \mathrm{kg}
\end{gathered}
$$

where $T$ is in $\mathrm{K}$.

Density of saturated water vapor in $\mathrm{kg} / \mathrm{m}^{3}$ as a function of $T$ in $K$ :

$$
\begin{aligned}
& \rho_{v s}=\frac{1}{194.4} \exp \left[6.374 \times 10^{-2}(T-273.15)-1.634 \times 10^{-4}(T-273.15)^{2}\right] \text { for } T>273.15 K \\
& \rho_{v s}=\frac{1000}{R_{w} T} \exp \left[C_{1} / T+C_{2}+C_{3} T+C_{4} T^{2}+C_{5} T^{3}+C_{6} T^{4}+C_{7} \ln (T)\right] \text { for } T \leq 273.15 K
\end{aligned}
$$

where $C_{1}=-5674.5359, C_{2}=-0.51523058, C_{3}=-9.677843 \times 10^{-3}, C_{4}=6.2215701 \times 10^{-7}$, 


$$
C_{5}=2.0747825 \times 10^{-9}, C_{6}=-9.484024 \times 10^{-13}, C_{7}=4.1635019
$$

The molecular diffusivity of water vapor in air $D_{v a}$ is defined as [67]:

$$
D_{v a}=\frac{1.858 \times 10^{-7} T^{1.5} \sqrt{\frac{1}{M_{w}}+\frac{1}{M_{a}}}}{P \cdot d_{v a}^{2} \cdot \Omega_{D}}
$$

where $M$ is the molecular weight $(\mathrm{g} / \mathrm{mol}), P(=293 / T)$ is the absolute pressure (in atm), $T$ is the absolute temperature, $d_{v a}=\left(d_{\text {air }}+d_{w}\right) / 2, d_{\text {air }}=3.617 \AA, d_{w}=2.649 \AA, M_{a}=29 \mathrm{~g} / \mathrm{mol}, M_{w}=18$ $\mathrm{g} / \mathrm{mol}$, and $\Omega_{\mathrm{D}}$ is the collision integral based on the Lennard-Jones potential which depends on force constant $\dot{\varepsilon}$ (=185.8278 $K$ for air and water). The actual value of $\Omega_{\mathrm{D}}$ can be interpolated from Table A 1 using $T$ and $\varepsilon^{\prime}$.

Table A1. Collision integral based on the Lennard-Jones potential [67]

\begin{tabular}{|c|c|c|c|c|c|c|c|c|c|c|c|}
\hline $\boldsymbol{T} / \boldsymbol{\varepsilon}$ & $\mathbf{0 . 3 0}$ & $\mathbf{0 . 3 5}$ & $\mathbf{0 . 4 0}$ & $\mathbf{0 . 4 5}$ & $\mathbf{0 . 5 0}$ & $\mathbf{0 . 5 5}$ & $\mathbf{0 . 6 0}$ & $\mathbf{0 . 6 5}$ & $\mathbf{0 . 7 0}$ & $\mathbf{0 . 7 5}$ & $\mathbf{0 . 8 0}$ \\
\hline $\boldsymbol{\Omega}_{\boldsymbol{D}}$ & 2.662 & 2.476 & 2.318 & 2.184 & 2.066 & 19.66 & 1.877 & 1.798 & 1.729 & 1.667 & 1.612 \\
\hline $\boldsymbol{T} / \boldsymbol{\varepsilon}$ & $\mathbf{0 . 8 5}$ & $\mathbf{0 . 9 0}$ & $\mathbf{0 . 9 5}$ & $\mathbf{1 . 0 0}$ & $\mathbf{1 . 0 5}$ & $\mathbf{1 . 1 0}$ & $\mathbf{1 . 1 5}$ & $\mathbf{1 . 2 0}$ & $\mathbf{1 . 2 5}$ & $\mathbf{1 . 3 0}$ & $\mathbf{1 . 3 5}$ \\
\hline $\boldsymbol{\Omega}_{\boldsymbol{D}}$ & 1.562 & 1.517 & 1.476 & 1.439 & 1.406 & 1.375 & 1.346 & 1.320 & 1.296 & 1.273 & 1.253 \\
\hline $\boldsymbol{T} / \boldsymbol{\varepsilon}$ & $\mathbf{1 . 4 0}$ & $\mathbf{1 . 4 5}$ & $\mathbf{1 . 5 0}$ & $\mathbf{1 . 5 5}$ & $\mathbf{1 . 6 0}$ & $\mathbf{1 . 6 5}$ & $\mathbf{1 . 7 0}$ & $\mathbf{1 . 7 5}$ & $\mathbf{1 . 8 0}$ & $\mathbf{1 . 8 5}$ & $\mathbf{1 . 9 0}$ \\
\hline $\boldsymbol{\Omega}_{\boldsymbol{D}}$ & 1.233 & 1.215 & 1.198 & 1.182 & 1.167 & 1.153 & 1.140 & 1.128 & 1.116 & 1.105 & 1.094 \\
\hline $\boldsymbol{T} / \boldsymbol{\varepsilon}$ & $\mathbf{1 . 9 5}$ & $\mathbf{2 . 0 0}$ & $\mathbf{2 . 1 0}$ & $\mathbf{2 . 2 0}$ & $\mathbf{2 . 3 0}$ & $\mathbf{2 . 4 0}$ & $\mathbf{2 . 5 0}$ & $\mathbf{2 . 6 0}$ & $\mathbf{2 . 7 0}$ & $\mathbf{2 . 8 0}$ & $\mathbf{2 . 9 0}$ \\
\hline $\boldsymbol{\Omega}_{\boldsymbol{D}}$ & 1.084 & 1.075 & 1.057 & 1.041 & 1.026 & 1.012 & .9996 & .9878 & .9770 & .9672 & .9576 \\
\hline $\boldsymbol{T} / \boldsymbol{\varepsilon}$ & $\mathbf{3 . 0 0}$ & $\mathbf{3 . 1 0}$ & $\mathbf{3 . 2 0}$ & $\mathbf{3 . 3 0}$ & $\mathbf{3 . 4 0}$ & $\mathbf{3 . 5 0}$ & $\mathbf{3 . 6 0}$ & $\mathbf{3 . 7 0}$ & $\mathbf{3 . 8 0}$ & $\mathbf{3 . 9 0}$ & $\mathbf{4 . 0 0}$ \\
\hline $\boldsymbol{\Omega}_{\boldsymbol{D}}$ & .9490 & .9406 & .9328 & .9256 & .9186 & .9120 & .9058 & .8998 & .8942 & .8888 & .8836 \\
\hline $\boldsymbol{T} / \boldsymbol{\varepsilon}$ & $\mathbf{4 . 1 0}$ & $\mathbf{4 . 2 0}$ & $\mathbf{4 . 3 0}$ & $\mathbf{4 . 4 0}$ & $\mathbf{4 . 5 0}$ & $\mathbf{4 . 6 0}$ & $\mathbf{4 . 7 0}$ & $\mathbf{4 . 8 0}$ & $\mathbf{4 . 9 0}$ & $\mathbf{5 . 0 0}$ & $\mathbf{6 . 0 0}$ \\
\hline $\boldsymbol{\Omega}_{\boldsymbol{D}}$ & .8788 & .8740 & .8694 & .8652 & .8610 & .8568 & .8530 & .8492 & .8456 & .8422 & .8124 \\
\hline $\boldsymbol{T} / \dot{\boldsymbol{\varepsilon}}$ & $\mathbf{7 . 0 0}$ & $\mathbf{8 . 0 0}$ & $\mathbf{1 0 . 0}$ & $\mathbf{2 0 . 0}$ & $\mathbf{3 0 . 0}$ & $\mathbf{4 0 . 0}$ & $\mathbf{5 0 . 0}$ & $\mathbf{6 0 . 0}$ & $\mathbf{7 0 . 0}$ & $\mathbf{8 0 . 0}$ & $\mathbf{9 0 . 0}$ \\
\hline $\boldsymbol{\Omega}_{\boldsymbol{D}}$ & .7896 & .7712 & .7424 & .6640 & .6232 & $\mathbf{. 5 9 6 0}$ & .5756 & .5596 & .5464 & .5352 & .5256 \\
\hline
\end{tabular}

\section{A.2 - Soil Moisture Retention}

Other than loamy sand (e.g., QC2), the moisture retention (or soil water characteristic curve is estimated by van Genuchten [68] as follows:

$$
\Theta=\frac{\theta-\theta_{r}}{\theta_{s}-\theta_{r}}=\left(1+|\alpha \psi|^{n}\right)^{-m}
$$

where $\theta_{s}$ is the saturated water content $\left(\mathrm{m}^{3} / \mathrm{m}^{3}\right) ; \theta_{r}$ is the residual water content $\left(\mathrm{m}^{3} / \mathrm{m}^{3}\right) ; m=1-$ $1 / n$; and $\alpha, m, n$ are fitting parameters (more can be found from Grant et al. [52]). 
The soil moisture retention curve correlation for the loamy sand with a reference temperature of $25^{\circ} \mathrm{C}$ was published by Noborio et al. [69] as follows:

$$
\begin{gathered}
\psi=\frac{1}{g} \sqrt{\frac{1-\Theta}{0.01223}} \text { for } \Theta \geq 0.56362 \\
\psi=\frac{1}{g}\left(0.551 \Theta^{-2.1}+4.136488\right) \text { for } \Theta<0.56362
\end{gathered}
$$

where $g$ is the gravitational constant $\left(\sim 9.807 \mathrm{~m} / \mathrm{s}^{2}\right)$

\section{A.3 - Hydraulic Conductivity}

If measured data is known for the range of moisture contents, a good approximation can be found by curve-fitting the data using the least square method. Otherwise, fairly good models for hydraulic conductivity for soil are [52], [69] and [28]:

$$
\begin{gathered}
K(\Theta)=K_{\text {sat }} \Theta^{0.5}\left[1-\left(1-\Theta^{1 / m}\right)^{m}\right]^{2} \\
K(\psi)=K_{\text {sat }} \frac{\left[1-|\alpha \psi|^{n-1}\left(1+|\alpha \psi|^{n}\right)^{-m}\right]^{2}}{\left(1+|\alpha \psi|^{n}\right)^{m / 2}} \\
K=K_{\text {sat }} \Theta^{11.39} \quad \text { for } 0.375<\theta \leq \theta_{s} \\
K=0.71356 K_{\text {sat }} \Theta^{5.3817} \quad \text { for } 0.239<\theta \leq 0.375 \\
K=4.5444 K_{\text {sat }} \Theta^{8.9775} \quad \text { for } 0.0<\theta \leq 0.239
\end{gathered}
$$

where $K_{\text {sat }}$ is saturated hydraulic conductivity $(\mathrm{m} / \mathrm{s})$ and Eq. A.15 is not good for loamy sands

\section{A.4 - Temperature Dependency}

To account for the temperature dependency, a reference temperature (which can be at room temperature) can be applied to better obtain the matric potential and hydraulic conductivity using the surface-tension viscous-flow (STVF) approach as follow ([70] and [71]):

$$
\psi\left(T, \theta_{l}\right)=\frac{\sigma_{w}(T)}{\sigma_{w}\left(T_{r e f}\right)} \psi\left(T_{r e f}, \theta_{l}\right)
$$




$$
K\left(T, \theta_{l}\right)=\frac{v\left(T_{r e f}\right)}{v(T)} K\left(T_{r e f}, \theta_{l}\right)
$$

\section{A.5 - Thermal Conductivity}

There are many models for thermal conductivity of soils. One model is from de Vries [53] as below:

$$
k=\frac{x_{w} k_{w}+\xi_{p} x_{p} k_{p}+\sum_{i=1}^{n} \xi_{i} x_{i} k_{i}}{x_{w}+\xi_{p} x_{p}+\sum_{i=1}^{n} \xi_{i} x_{i}}
$$

where $x$ is the volume fraction of a component in the soil, subscript $p$ means pertaining to pore, and $\xi$ is the ratio of the average temperature gradient in the constituent and is approximated by:

$$
\xi_{i}=\frac{1}{3} \sum_{i=a, b, c}\left[1+\left(\frac{k_{i}}{k_{w}-1}\right) g_{i}\right]^{-1}
$$

The values of $\mathrm{g}_{\mathrm{a}}, g_{b}$, and $g_{c}$ depend on the ratios of the axes of the grains and they sum to unity. For a sphere, they are equal to $1 / 3$. See Table A2 for the values used in this work.

Table A2. Physical and thermal properties of soil constituents [28]

\begin{tabular}{|l|c|c|c|c|}
\hline Soil constituent & $k(\mathrm{~W} / \mathrm{m} \cdot \mathrm{K})$ & $\rho\left(\mathrm{kg} / \mathrm{m}^{3}\right)$ & $C_{p}(\mathrm{~J} / \mathrm{kg} \cdot \mathrm{K})$ & $g_{i}$ \\
\hline Quartz & Eq. A.22 & 2,650 & 731.5 & 0.144 \\
\hline Other minerals & 2.93 & 2,650 & 731.5 & 0.144 \\
\hline Organic material & 0.25 & 1,300 & 1,923 & 0.5 \\
\hline Water & Eq. A.2 & Eq. A.3 & 4,180 & N/A \\
\hline Air & Eq. A.1 & 1.2 & 1,007 & N/A \\
\hline
\end{tabular}

The effective thermal conductivity of the gas-filled pores, which contains air and vapor, can be written as [28]:

$$
k_{p}=k_{a}+k_{v}
$$

where $k_{a}$ is the thermal conductivity of air and $k_{v}$ is the effective thermal conductivity of vapor diffusion which is given by 


$$
k_{v}=h_{f g} D_{v a} \varphi \frac{d \rho_{v s}}{d T}
$$

The effective thermal conductivity $k_{\text {eff }}$ of the moist soil in Eq. $2.5 \mathrm{~b}$ can be evaluated using Eq. A.18 with $k_{p}$ from Eq. A.20.

The thermal conductivity of quartz is given by [28]:

$$
k_{\text {quartz }}=9.051-0.02659(T-273.15) \mathrm{W} / \mathrm{m} \cdot \mathrm{K}
$$

where $T$ is in $\mathrm{K}$.

The volumetric heat capacity of soil can be calculated as:

$$
C_{\text {soil }}=x_{w} \rho_{w}\left(C_{p}\right)_{w}+x_{a} \rho_{a}\left(C_{p}\right)_{a}+\sum_{i=1}^{n} x_{i} \rho_{i}\left(C_{p}\right)_{i}
$$

where subscripts $w$ and $a$ means water and air, respectively, and $n$ is the number of constituents that form the solid part of the soil.

\section{A.6 - Other Properties and Parameter Definitions}

The total potential (or head of water) in the soil is:

$$
\Phi=\psi+z
$$

Another formulation for the matric potential can be expressed as [72]:

$$
\psi(\theta, T)=\psi\left(\theta, T_{r e f}\right) e^{-C_{\psi}\left(T-T_{r e f}\right)}
$$

where $C_{\psi}=\left.\frac{1}{\psi} \frac{\partial \psi}{\partial T}\right|_{\theta}=0.0068 \mathrm{~K}^{-1}$.

The flow velocity of water through the soil is:

$$
\boldsymbol{u}_{l}=-K(\psi) \nabla \Phi
$$

\section{A.7 - More about COMSOL Simulations in Chapter 6}

The thermal conductivities of quartz, silt and clay can be expressed as [29]:

$$
\begin{gathered}
k_{\text {quartz }}=-5.31 \times 10^{-8} T^{3}+6.55 \times 10^{-5} T^{2}-3.04 \times 10^{-2} \mathrm{~T}+8.93 \mathrm{~W} / \mathrm{m} \cdot \mathrm{K} \\
k_{\text {silt }}=9.52 \times 10^{-4} \mathrm{~T}+0.2286 \mathrm{~W} / \mathrm{m} \cdot \mathrm{K}
\end{gathered}
$$




$$
k_{\text {clay }}=9.23 \times 10^{-4} \mathrm{~T}+0.2281 \mathrm{~W} / \mathrm{m} \cdot \mathrm{K}
$$

where $T$ is in ${ }^{\circ} \mathrm{C}$.

Due to many variations of sand, the average thermal conductivity of quartz is assumed to be that of sand for COMSOL simulations.

The specific heat capacities of quartz, silt and clay can be expressed as [29]:

$$
\begin{gathered}
\left(C_{p}\right)_{\text {quartz }}=2.09 \mathrm{~T}+655 \mathrm{~J} / \mathrm{kg} \cdot \mathrm{K} \\
\left(C_{p}\right)_{\text {silt }}=3.94 \times 10^{-5} \mathrm{~T}^{3}+2.00 \times 10^{-2} \mathrm{~T}^{2}+4.59 \mathrm{~T}+744 \mathrm{~J} / \mathrm{kg} \cdot \mathrm{K} \\
\left(C_{p}\right)_{\text {clay }}=4.49 \mathrm{~T}+819 \mathrm{~J} / \mathrm{kg} \cdot \mathrm{K}
\end{gathered}
$$

where $T$ is in ${ }^{\circ} \mathrm{C}$.

Table A3 shows the estimated diameters and masses of sand, silt, and clay. By assuming spherical particles, the solid density of sand, silt, and clay can be calculated as:

$$
\rho_{i}=m_{i} \times\left(\frac{4}{3} \pi r^{3}\right)^{-1}
$$

where $m$ is the mass, subscript $i$ represents sand, silt, or clay, and $r$ is the particle's radius.

Table A3. Estimated diameters and masses of sand, silt, and clay [73]

\begin{tabular}{|l|c|c|c|}
\hline Particle & Sand & Silt & Clay \\
\hline Diameter (m) & $5 \times 10^{-5}$ & $2 \times 10^{-6}$ & $2 \times 10^{-6}$ \\
\hline Mass (kg) & $1.77 \times 10^{-10}$ & $1.13 \times 10^{-14}$ & $8.48 \times 10^{-18}$ \\
\hline
\end{tabular}

Using Eq. A.29, the solid densities of sand, silt, and clay respectively are $2704 \mathrm{~kg} / \mathrm{m}^{3}, 2698$ $\mathrm{kg} / \mathrm{m}^{3}$, and $2025 \mathrm{~kg} / \mathrm{m}^{3}$. As reported by Tarnawski et al. [57], the solid density of Sable sand (NS4), which contains sand only, is $2662 \mathrm{~kg} / \mathrm{m}^{3}$. Consequently, the solid densities of silt and clay calculate from [73] are reliable.

The grain size distribution (GSD) is expressed as [57]: 


$$
G S D_{i}=\frac{m_{i}}{m_{t o t}}=\frac{\rho_{i} \cdot \forall_{i}}{\rho_{b} \cdot \forall_{t o t}}=\frac{\rho_{i}}{\rho_{b}} \cdot x_{i}
$$

where $\rho_{b}$ is the bulk density of the soil, subscript $i$ represents sand, clay, or silt, $\rho_{i}$ is the solid density of component $i$, and $x$ is the volume fraction of a mineral in the soil.

From Eq. A.30, the volume fraction of a component in the soil can be written as:

$$
x_{i}=G S D_{i} \cdot \frac{\rho_{b}}{\rho_{i}}
$$

Matilda soil is experimentally measured to have a bulk density of $1480 \mathrm{~kg} / \mathrm{m}^{3}$. Because Matilda soil contains (in terms of mass \%) 70.9\% sand, $25.5 \%$ silt, and 3.6\% clay [57], the volume fractions of sand, silt, and clay respectively are $38.8 \%, 14.0 \%$, and $2.63 \%$ using Eq. A.31. The volume fractions are entered into the Heat Transfer in Porous Media module and volume average method is used to calculate the effective thermal conductivity of the soil in COMSOL. Darcy model was used with permeability $\kappa$ of $5 \times 10^{-12} \mathrm{~m}^{2}$ and particle size of $0.2 \mathrm{~mm}$. Buoyancy term is included.

\section{A.8 - More Thermal Properties for Section 7.1}

Eq. A.23 can be re-written as:

$$
C_{\text {soil }}=\theta_{w} \rho_{w}\left(C_{p}\right)_{w}+\left(\eta-\theta_{w}\right) \rho_{a}\left(C_{p}\right)_{a}+(1-\eta) \rho_{s}\left(C_{p}\right)_{s}
$$

Re-arranging Eq. A.32, the thermal capacity of the solid part of the soil can be expressed as:

$$
\rho_{s}\left(C_{p}\right)_{s}=\frac{C_{\text {soil }}-\theta_{w} \rho_{w}\left(C_{p}\right)_{w}-\left(\eta-\theta_{w}\right) \rho_{a}\left(C_{p}\right)_{a}}{1-\eta}
$$

In Section 7.1, the pure heat conduction model (PHCM) is used, so the water content $\theta_{w}$ is set to be constant and depends only on the porosity $\eta$. The specific heat capacities and densities of water and air as functions of temperature can be interpolated using standard tables for air and water

properties. The thermal capacity of the soil, $C_{\text {soil }}$, as a function of temperature can be found in 
Sections 4.2 and 4.3 for $\mathrm{SR} \approx 0.50$. As a result, the thermal capacity of the solid part of the soil, $\rho_{s}\left(C_{p}\right)_{s}$, as a function of temperature can be obtained and is independent of the moisture content.

Substituting Eq. A.33 into Eq. A.26, the thermal capacity of wet soil $(\mathrm{SR} \approx 0.25)$ can be obtained as a function of temperature.

A simplified equation for the thermal conductivity of the soil can be expressed as:

$$
k_{\text {soil }}=\theta_{w} k_{w}+\left(\eta-\theta_{w}\right) k_{a}+(1-\eta) k_{s}
$$

Similar to the thermal capacity, the thermal conductivity of the soil with $\mathrm{SR} \approx 0.25$ can be obtained. Appendix A.1 describes the thermal conductivities of air and water as functions of temperature. 


\section{B - More about Matlab Programming}

To create a customized function in Matlab, the procedures (for Windows version) are:

1. Open Matlab and click on New Script in the Home tab to see the Editor window (Fig. B1)

2. Type in the desired function's name with its inputs (e.g., Fig. B1). Insert as many equations and/or commands in the function as one wishes. The main program will use the value from the parameter result when the main program calls the function. While the function is being programmed, any part can be selected and then the 'F9' button can be pressed so that the Command window of Matlab can evaluate the part. A semicolon at the end of a line supresses the outcome of the line in the Command window

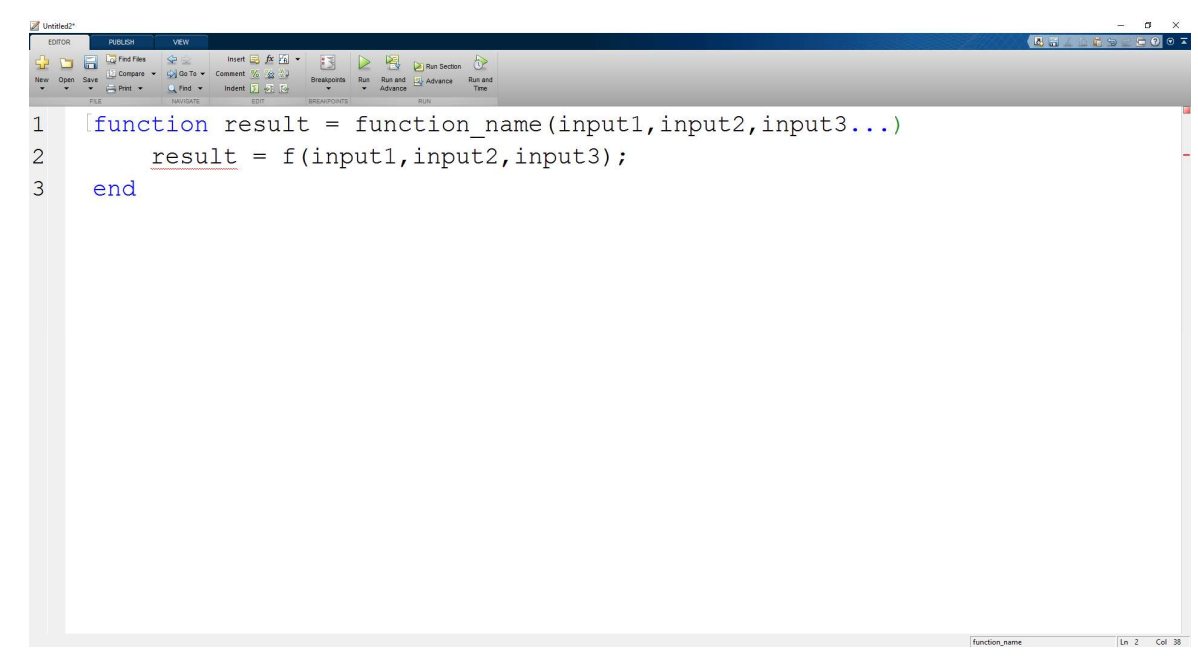

Figure B1. Example of Matlab function.

3. The customized function must be saved in C: $\backslash$ Users $\backslash$ UserName $\backslash$ Documents $\backslash M A T L A B \backslash$ directory so that Matlab can locate and call the customized function

There are many situations where data in an Excel files needs to be read. For example, the temperatures at nodes A and B (bottom and top of soil column respectively) in Section 2.3 vary with time but need to be known at all time, so the command xlsread in Matlab is used. The syntanx and procedures for using $x l s r e a d$ are:

1. FileName $={ }^{\prime E x c e l F i l e D i r e c t o r y} \mid$ ExcelFileName.xlsx'

2. $\quad$ variable $=x$ lsread(FileName,'DataSheet','CellRange') 
After telling Matlab to read data from all Excel files, calculations are started. Then, the desired calculated results can be written to Excel files. The command used to write to Excel files is $x l s w r i t e$ in Matlab. The syntax and procedures for xlswrite are:

1. DesiredFileName $={ }^{\prime}$ ExcelFileDirectory $\backslash$ ExcelFileName.xlsx'

2. xlswrite(DesiredFileName,CalculatedValues,'DataSheet','CellRange')

One needs to be cautious when using xlswrite as the command can overwrite the existing data or information in any cell in the Excel file, so it is recommended that the CellRange should be coded such that the corresponding cell(s) in the Excel file is/are empty.

Since the heat pulse method is applied in this thesis, the method to obtain the thermal conductivity and volumetric heat capacity of the soil follows that of Knight et al. [54] who was generous enough to share the Matlab code. However, the code (labeled as icpc.m and icpcinv.m) could not be used to estimate the thermal properties from the experimental (temperature) data. The original code was to plot temperature response from known thermal properties of the soil and the heat pulse probe. As a result, the code was modified so that curve-fitting techniques can be applied.

The built-in fminsearch function of Matlab was used to estimate the thermal properties of the soil using the temperature data from the experiments and the modified code. fminsearch finds the minimum of a scalar function of several variables at an initial estimate, which is generally referred to as unconstrained nonlinear optimization. The curve-fitting method using fminsearch function of Matlab is:

1. Develop or come up with a fitting function that resembles the experimental data as $f$ fit. $k$ soil as $x(1)$ and $H C \_$soil as $x(2)$ are the inputs to calculate the values of thermal conductivity and heat capacity of the soil

2. Define the independent (time) row of data as $x$ data and dependent (temperature) row of data as ydata. Experimental data from an Excel file can be read for the independent and dependent rows. If the read Excel data are in the form of a column with many data, transpose the column

3. Define $f u n c 1=@(x) \operatorname{sum}\left(\left(y \operatorname{data}(1,:)-f \_f i t(\right.\right.$ input arguments $\left.\left.)\right) . \wedge 2\right)$ where input arguments include $x(1)$ and $x(2)$ together with other values so that $f$ fit can output a row of values. $y \operatorname{data}(1,:)-f$ fit(input arguments) gives a row of difference values between the $y$ data and 
$f_{f} f i t$. The dot before the power $2($ i.e.,.$\wedge 2)$ is to tell Matlab to use each difference value between $y d a t a$ and $f_{-}$fit for the square. Without the dot, Matlab by default will do matrix multiplication with the row of difference values and give error because matrix dimensions do not match

4. $\quad x$ guess $=$ [value 1 value2] where value 1 and value 2 are reasonably guessed values of soil's thermal conductivity and heat capacity respectively

5. Tell Matlab to calculate the thermal properties: $[x 1$ fminres $]=$ fminsearch $\left(\right.$ fun $1, x \_$guess $)$

6. $k_{-}$soil $=x l(1)$

7. HC_soil $=x 1(2)$ 


\section{C-Experimental Apparatus and Methodology by Hedayati-Dezfooli [27]}

\section{C.1 - Experimental Apparatus}

- Designs of major parts and components by Hedayati-Dezfooli are shown from Figs. C1 to C7.

- The apparatus was very similar to this thesis but had much less covering insulation.

- The computer code in the CRBasic Editor to collect the experimental data from the data acquisition (DAQ) system to the computer was limited to collecting data for three hours. In addition, the code had many 'if' conditions to turn on the heat pulse at specific times. There was no loop (e.g., 'for' and 'while' loops) to automatically and periodically turn on the heat pulse.

- The water flow from the water baths could not be stopped as desired since the flow switch was broken before the experiments for this thesis started.

- The heat flux meter was a round thin plate $(\varnothing 60 \mathrm{~mm}$ by $0.6 \mathrm{~mm})$ that had T-type thermocouples and could measure the heat flux over its surface area by sending electrical voltage to the DAQ system. The electrical voltage was then converted to $\mathrm{W} / \mathrm{m}^{2}$ by the conversion factor of $15 \mu \mathrm{V}$ per $1 \mathrm{~W} / \mathrm{m}^{2}$.

\section{C.2 - Experimental methodology}

- Thermo-time domain reflectometry (T-TDR) was used to obtain the thermal conductivity, volumetric heat capacity, and moisture content of the soil. T-TDR is a combination of the heat pulse method and the time domain reflectometry.

- The heat pulse method used line heat source theory to obtain the thermal conductivity and volumetric heat capacity of the soil. The theory does not consider the construction of the probe.

- The time domain reflectometry is a method to measure the moisture content of the soil by sending electromagnetic pulse waves through the soil. The travelling velocity of the waves depends on the soil's dielectric constant which is mainly influenced by the water content of the soil.

- The soil's porosity at the dry state was assumed to be the same as that at the moist state. 
$\frac{0}{\square}$
$\leq$
$\frac{5}{\square}$
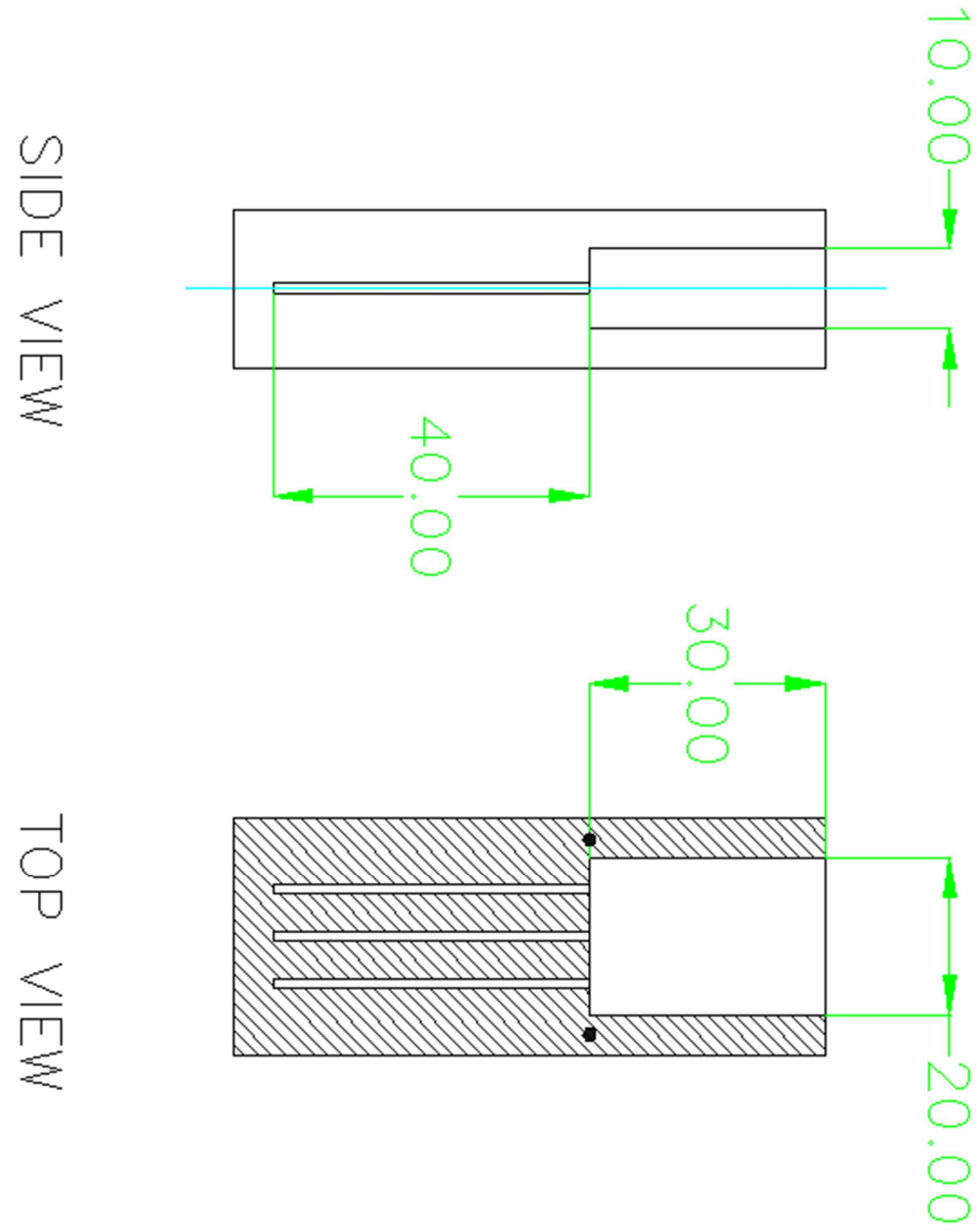

$\infty$
$D$
$\lambda$
$\leq$
$\frac{11}{2}$

Needle's center-tocenter distance: $6 \mathrm{~mm}$

Figure C1. Design of heat pulse probe and its mold 


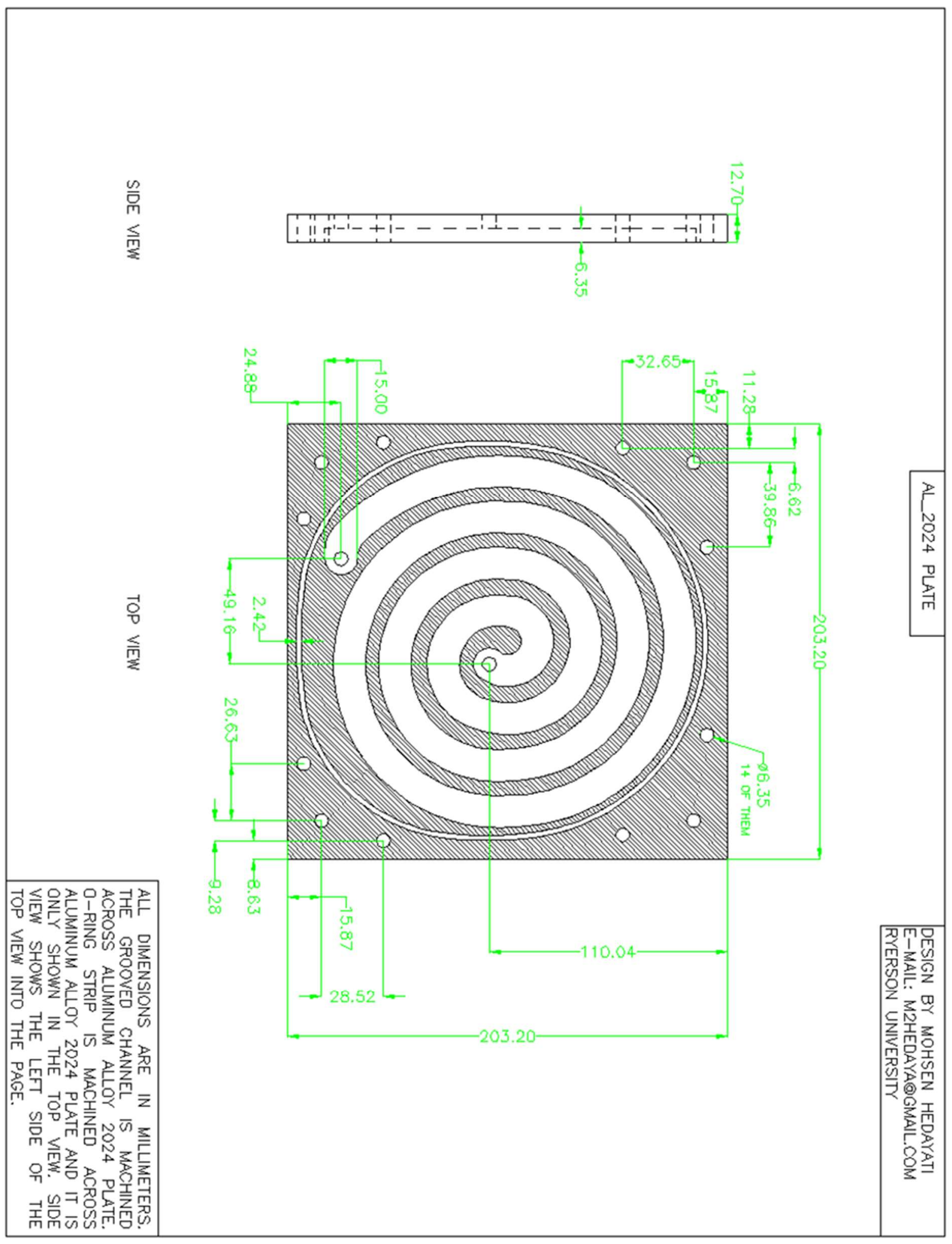

Figure C2. Heating/cooling plate design 


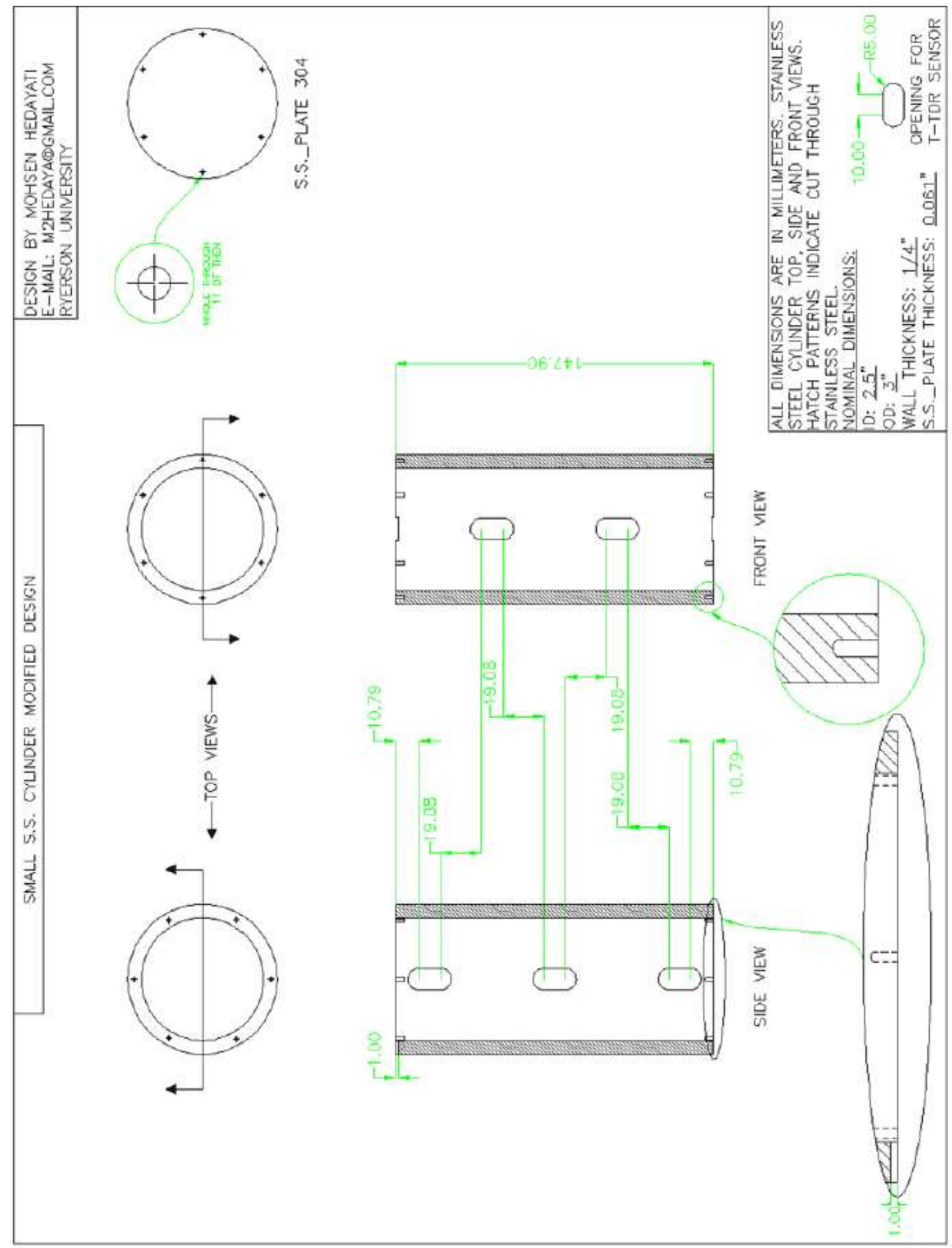

Figure C3. Design of soil column - Part I 


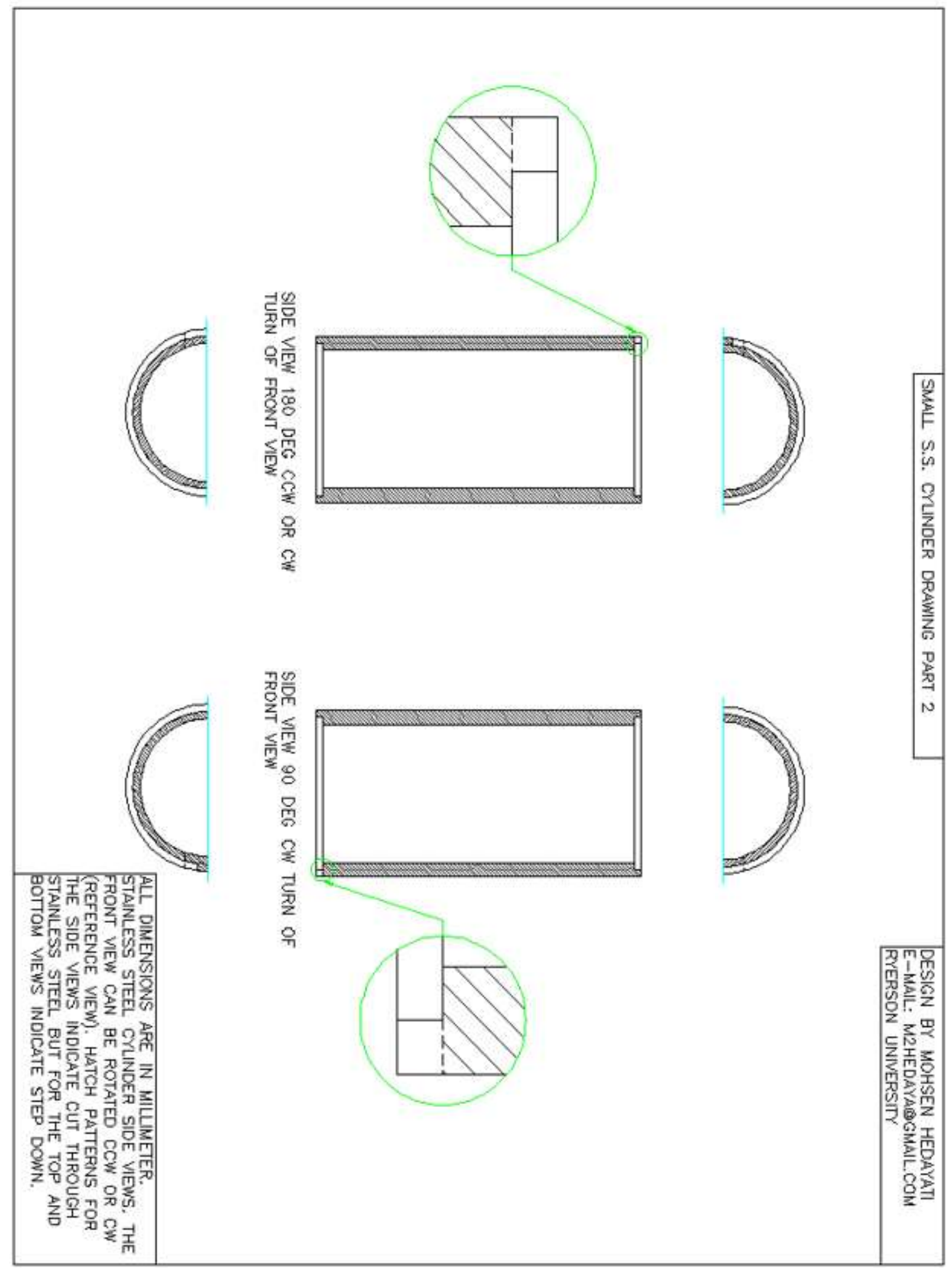

Figure C4. Design of soil column-Part II 


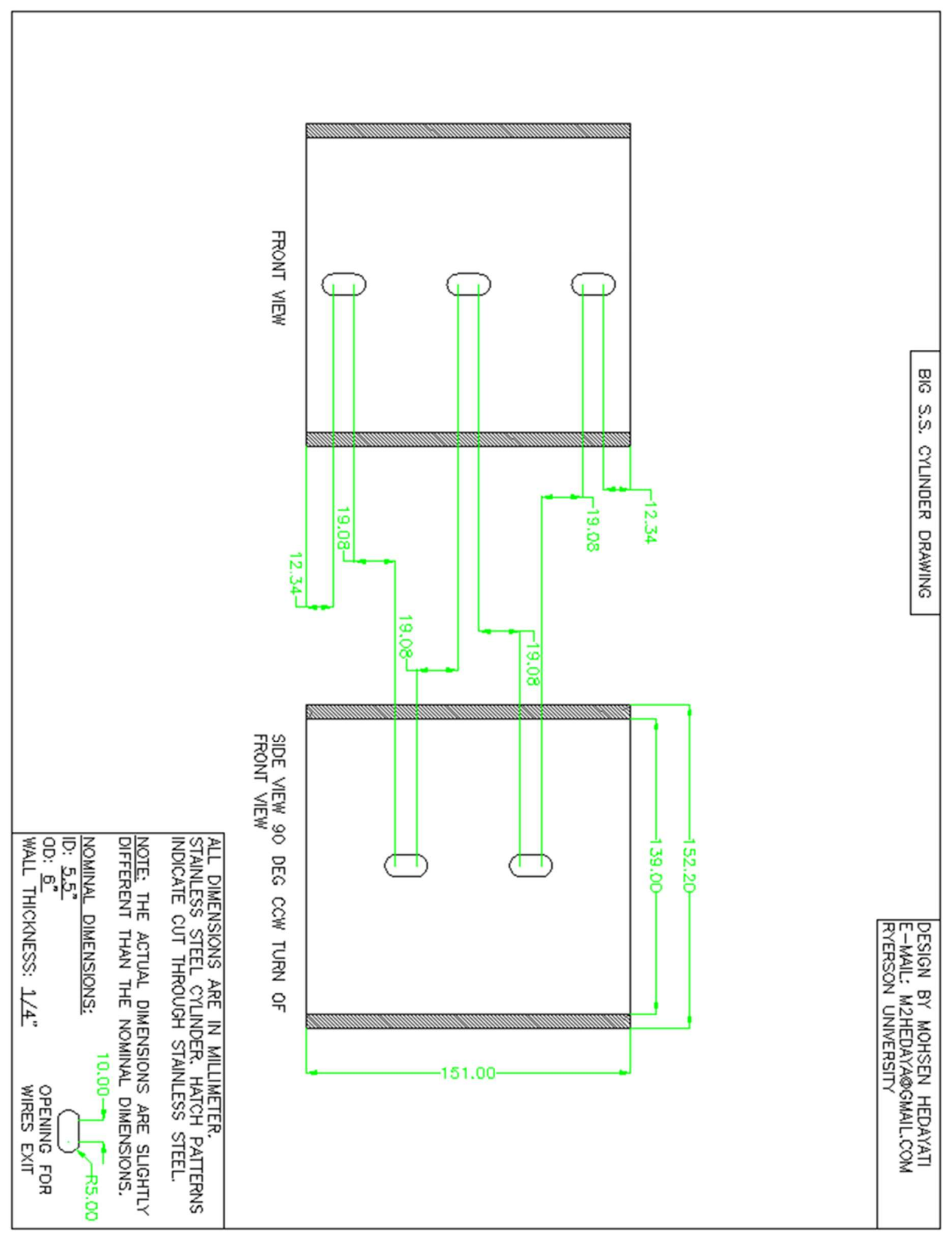

Figure C5. Design of outer container 


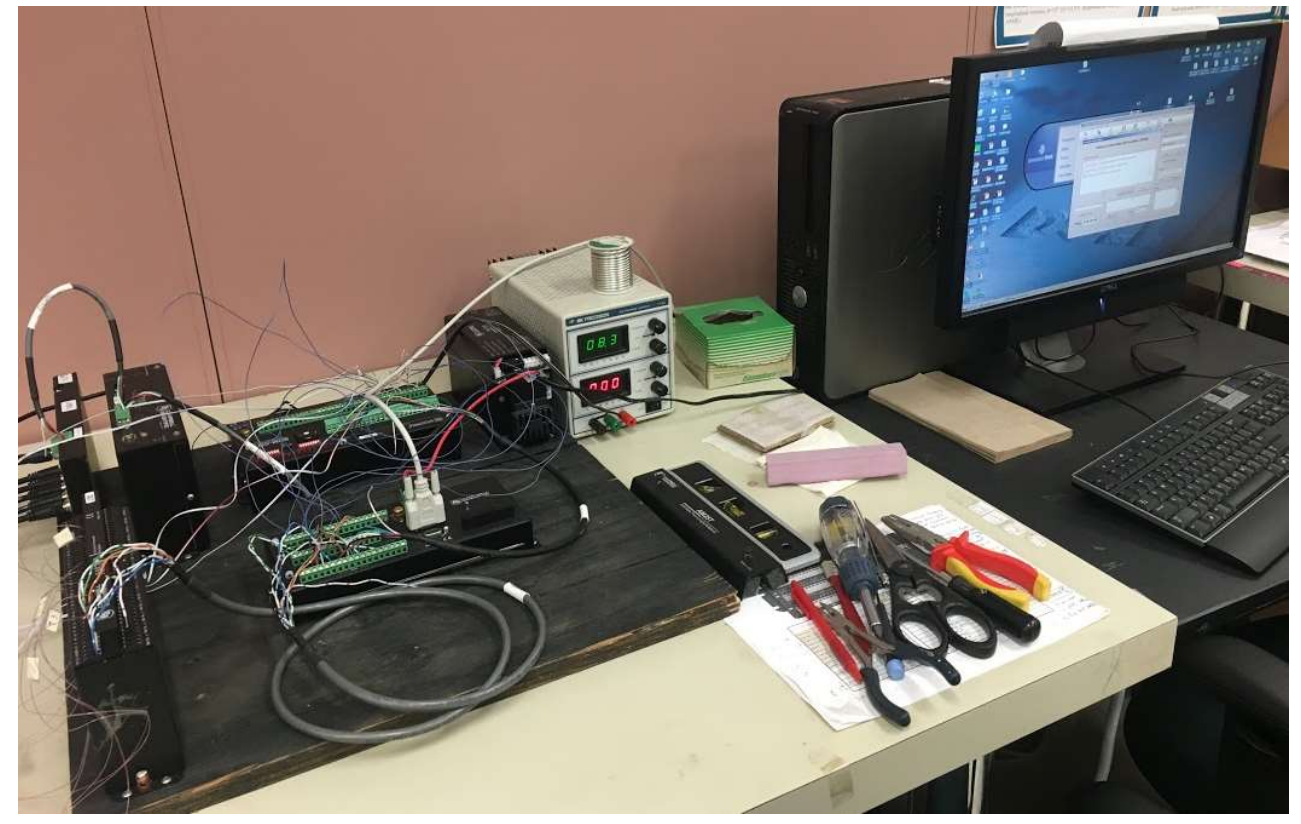

Figure C6. Campbell Scientific digital system and DC power supply connected to the PC via 8 conductor cable from datalogger (CR-1000).

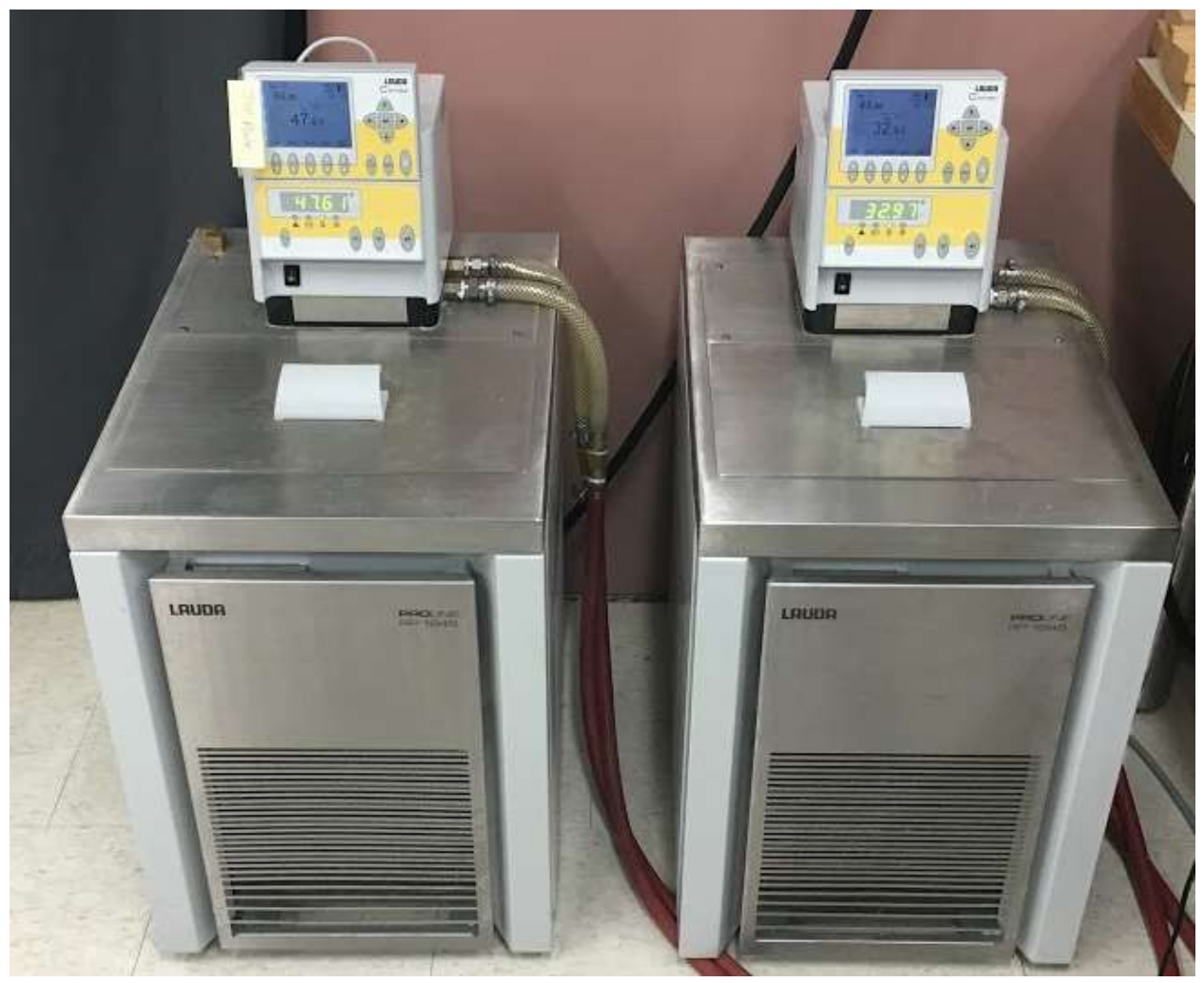

Figure C7. Lauda Proline RP1845 thermal baths. 


\section{D-Derivations of some Soil Parameters}

The dry bulk density of the soil (including the soil particles' and pore's spaces) in the beaker is calculated as:

$$
\rho_{b}=\frac{m_{d r y_{-} \text {soil }}}{\forall_{\text {soil }}}
$$

where $\forall_{\text {soil }}=600 \mathrm{~mL}$ (i.e., the total volume of the soil in the beaker) and $m_{d r y_{-} s o i l}$ is the mass of the dry soil in the beaker.

The porosity of the soil in the beaker is:

$$
\eta_{\text {beaker }}=1-\left(\frac{\rho_{b}}{\rho_{s}}\right)_{\text {beaker }}=\frac{\forall_{\text {pore }}}{\forall_{\text {soil }}}
$$

where $\rho_{s}$ is the solid particle density of the soil from Table 4.2.

The water volume in the soil in the beaker is:

$$
\forall_{w}=\forall_{\text {soil }} \cdot \frac{\forall_{\text {pore }}}{\forall_{\text {soil }}} \cdot \frac{\forall_{w}}{\forall_{\text {pore }}}=\forall_{\text {soil }} \cdot \eta_{\text {beaker }} \cdot S R
$$

where $S R=\forall_{w} / \forall_{\text {pore }}$ is the saturation ratio.

The water mass in the soil is:

$$
m_{w}=\rho_{w} \cdot \forall_{w}
$$

Inserting Eqs. D.1, D.2 and D.3 into Eq. D.4, the mass of the water in the soil column is:

$$
m_{w}=\rho_{w} \cdot \forall_{\text {soil }} \cdot\left(1-\frac{m_{\text {dry_soil }}}{\forall_{\text {soil }} \cdot \rho_{s}}\right) \cdot S R
$$

For a wet soil, assuming water is uniformly distributed among all dry soil particles, the mass of the wet soil in the beaker is: 


$$
m_{\text {wet_soil }}=m_{d r y_{-} s o i l}+m_{w}=m_{d r y_{\_} s o i l}+\frac{m_{w}}{m_{d r y_{-} s o i l}} \cdot m_{d r y_{-} s o i l}=\left(1+\frac{m_{w}}{m_{d r y_{-} s o i l}}\right) \cdot m_{d r y_{-} s o i l}
$$

Re-arranging Eq. D.6, the mass of the dry soil particles in the wet soil is:

$$
m_{d r y_{-} \text {soil }}=m_{\text {wet_s soil }} \cdot\left(1+\frac{m_{w}}{m_{d r y_{-} s o i l}}\right)^{-1}
$$

where $m_{w}$ and $m_{d r y_{-} s o i l}$ are respectively the masses of the water and dry soil in the beaker during the soil preparation processes. The bracketed term in Eq. D.7 is the ratio of the mass of wet soil to the mass of dry soil. With the assumption of uniformly prepared wet soil, the ratio will remain constant at any part of the wet soil.

After the soil column is filled with the wet soil, some wet soil is left over in the beaker. In order to determine the mass of dry soil particles in the soil column, Eq. D.7 can be used to calculate the mass of dry soil particles in the soil column as follows:

$$
m_{d r y}=m_{w e t} \cdot\left(1+\frac{m_{w}}{m_{d r y_{-} \text {soil }}}\right)^{-1}
$$

where $m_{w e t}$ is the mass of the wet soil in the soil column, which is the mass difference before and after the soil column is filled with the wet soil, and $m_{d r y}$ is the mass of dry soil particles of the wet soil in the soil column. The bracketed term in Eq. D.8 remains constant as in Eq. D.7 because the soil in the soil column is the same uniformly prepared wet soil.

From Eq. D.2, the overall porosity of the soil in the soil column can be expressed as:

$$
\eta_{\text {overall }}=1-\left(\frac{\rho_{b}}{\rho_{s}}\right)_{\text {column }}=1-\frac{1}{\rho_{s}}\left(\frac{m_{d r y}}{\forall_{\text {column }}}\right)
$$

where $\forall_{\text {column }}=470 \mathrm{~mL}$ is the internal volume of the soil column, excluding the space occupied by the needles of all five probes. $\rho_{s}$ is the solid particle density of the soil from Table 4.2. 
Inserting Eq. D.8 into Eq. D.9, the overall porosity of the soil in the soil column can be calculated as:

$$
\eta_{\text {overall }}=1-\frac{1}{\rho_{s}} \cdot \frac{m_{\text {wet }}}{\left(1+m_{w} / m_{d r y_{-} \text {soil }}\right) \cdot \forall_{\text {column }}}
$$

The water volume of the soil in the soil column is:

$$
\forall_{w}=\frac{m_{w}}{\rho_{w}}=\frac{m_{w e t}-m_{d r y}}{\rho_{w}}
$$

Assuming water is uniformly distributed in the soil and applying Eq. D.8 to Eq. D.11, the water volume in the soil column is:

$$
\forall_{w}=\frac{m_{w e t}}{\rho_{w}}\left(1-\frac{1}{1+\frac{m_{w}}{m_{d r y_{-} s o i l}}}\right)
$$

According to the definition, the total pore volume in the soil column is:

$$
\forall_{\text {pore }}=\eta_{\text {overall }} \cdot \forall_{\text {column }}
$$

The moisture content in the soil can be expressed as:

$$
\theta_{w}=\frac{\forall_{w}}{\forall_{\text {column }}}=\frac{m_{\text {wet }}}{\rho_{w} \cdot \forall_{\text {column }}}\left(1-\frac{1}{1+\frac{m_{w}}{m_{\text {dry_sol }_{\text {sol }}}}}\right)
$$

where $m_{\text {wet }}$ is the mass of the wet soil in the soil column, $\forall_{\text {column }}=470 \mathrm{~mL}$ is the internal volume of the soil column excluding the space occupied by the needles of all five probes, and $\rho_{w}$ is the water density. As mentioned before in Eq. D.8, the bracketed term, which contains $m_{w}$ and $m_{d r y_{-} s o i l}$, remains constant as in Eq. D.7 because the soil in the soil column is the same uniformly prepared wet soil. 
The volumetric heat capacity of a moist soil can be expressed as:

$$
C_{\text {soil }}=x_{a} C_{a}+x_{w} C_{w}+x_{s} C_{s}
$$

where $x$ is the volumetric content of the component, $x_{a}=\eta-\theta_{w}, x_{w}=\theta_{w}$ and $x_{s}=1-\eta$.

Re-arranging Eq. D.15, the moisture content in the soil can be expressed as:

$$
\theta_{w}=\frac{C_{\text {soil }}-x_{a} C_{a}-x_{s} C_{s}}{\rho_{w} c_{w}}
$$

where $\rho_{w} c_{w}=C_{w}$.

Since $x_{a} C_{a}$ is relatively much smaller than $x_{s} C_{s}$ and $C_{\text {soil }}$, the moisture content in the soil can be approximated as:

$$
\theta_{w} \approx \frac{C_{s o i l}-C_{d r y}}{\rho_{w} c_{w}}
$$

where $C_{d r y}=x_{s} C_{s}=(1-\eta) C_{s}$. 


\section{E-More Detailed Graphs}

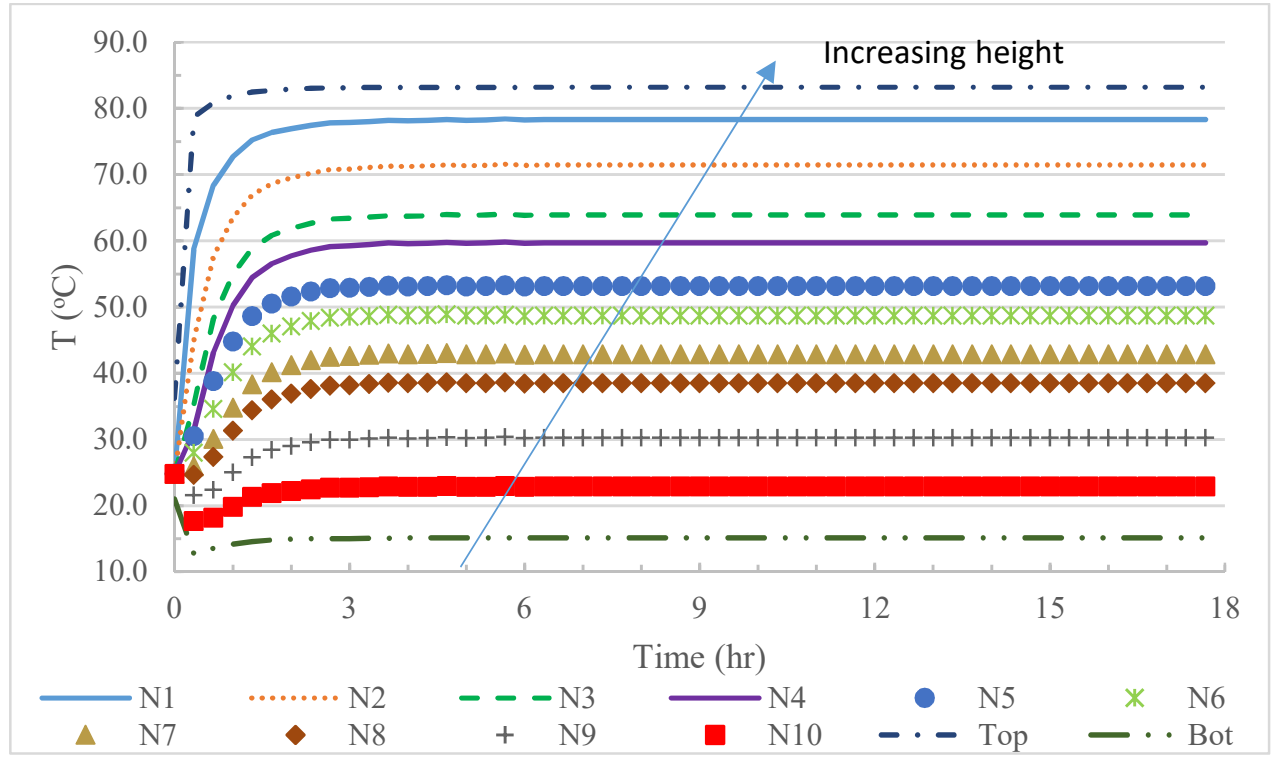

Figure E1. Simulated temperature at each needle (N\#) for the case of dry NB2 $(\eta=0.57)$ and vertical heating from top.

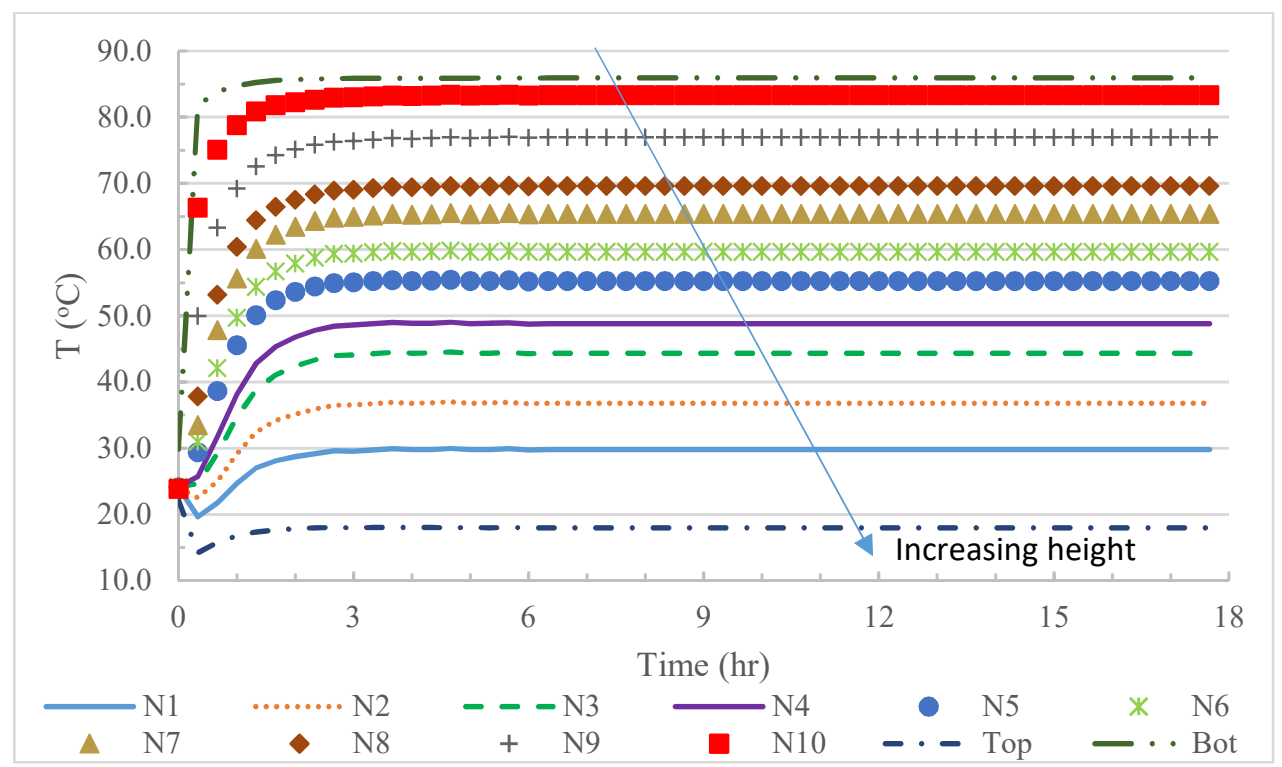

Figure E2. Simulated temperature at each needle (N\#) for the case of dry NB2 $(\eta=0.57)$ and vertical heating from bottom. 


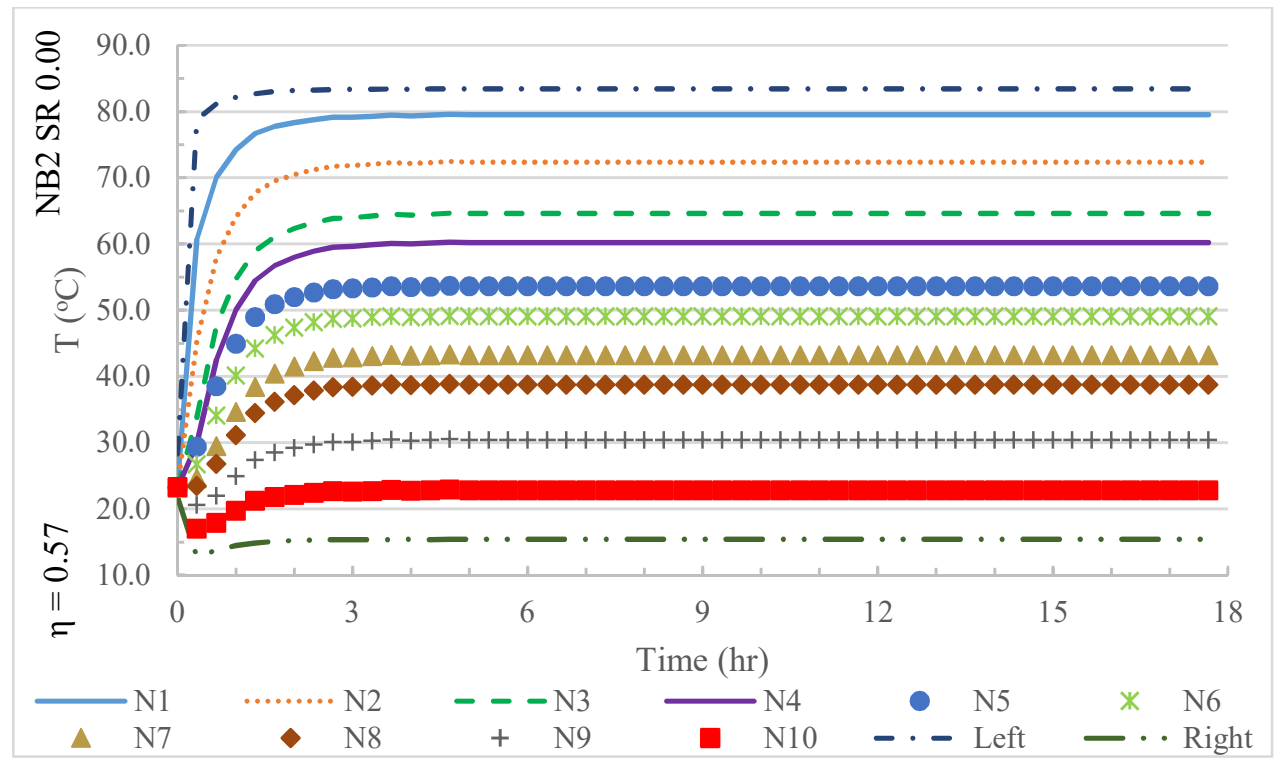

Figure E3. Simulated temperature at each needle (N\#) for the case of dry NB2 $(\eta=0.57)$ and horizontal heating. 


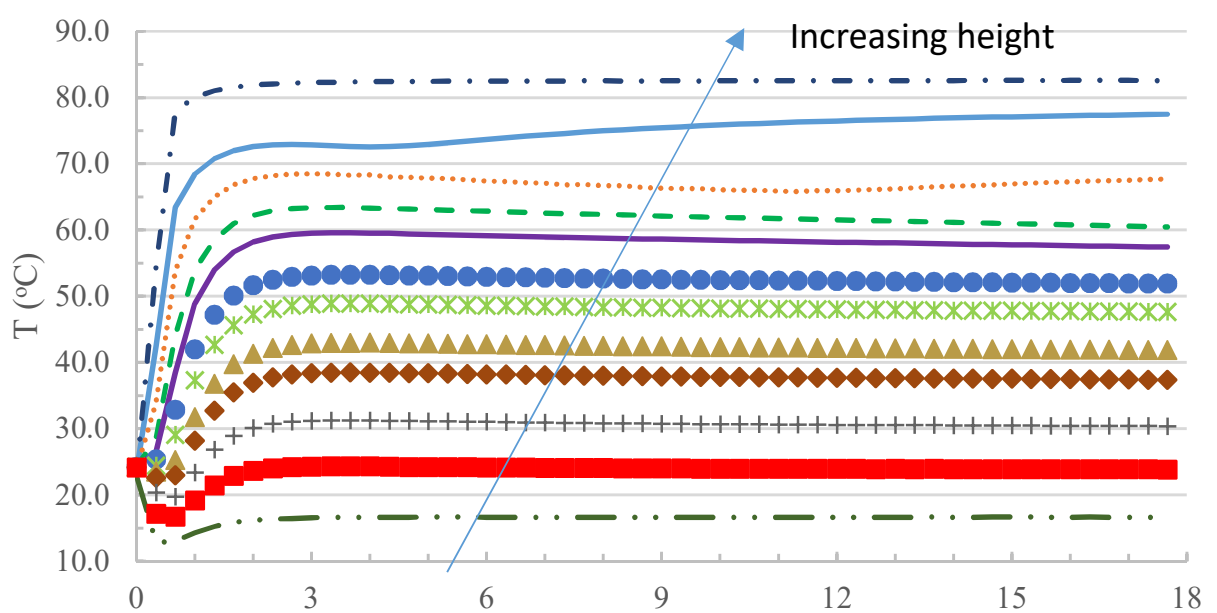

(a)

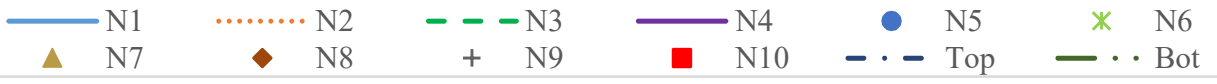

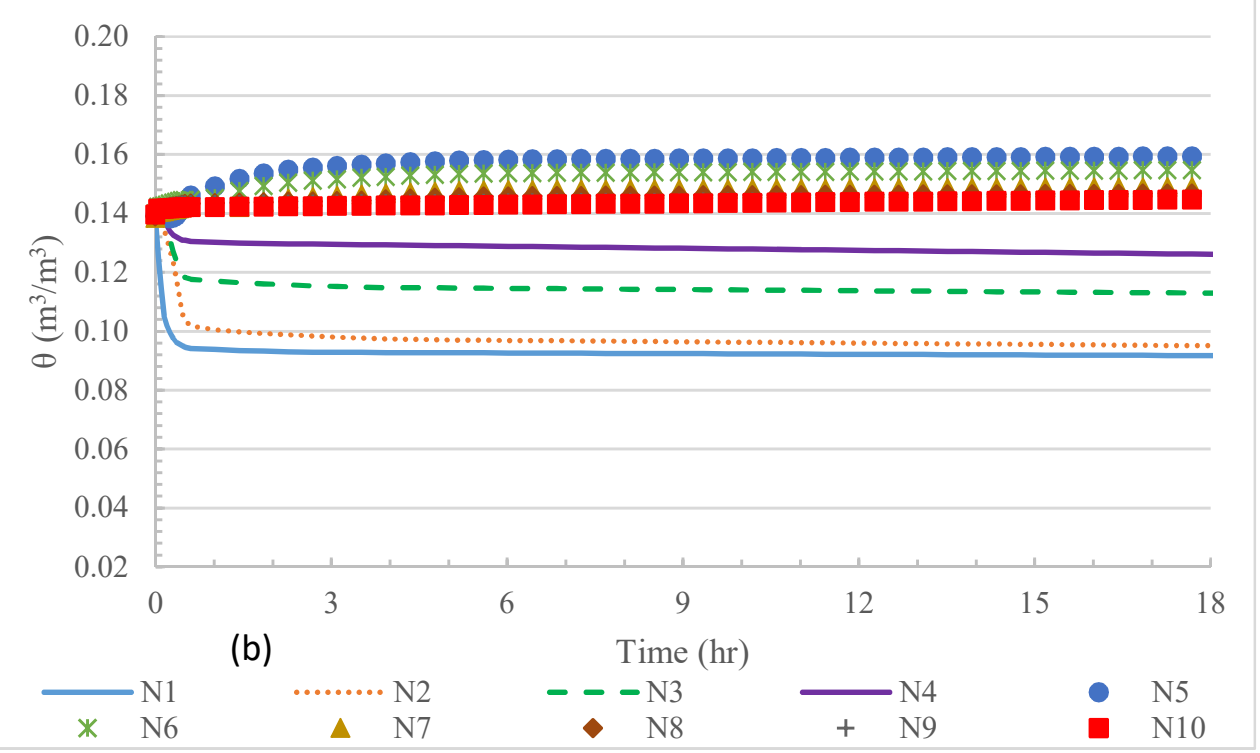

Figure E4. Simulated temperature and moisture content at each needle for the case of wet NB2 $(\eta=0.56, S R=0.25)$ and vertical heating from top. (a): temperature response. (b): moisture content response. 


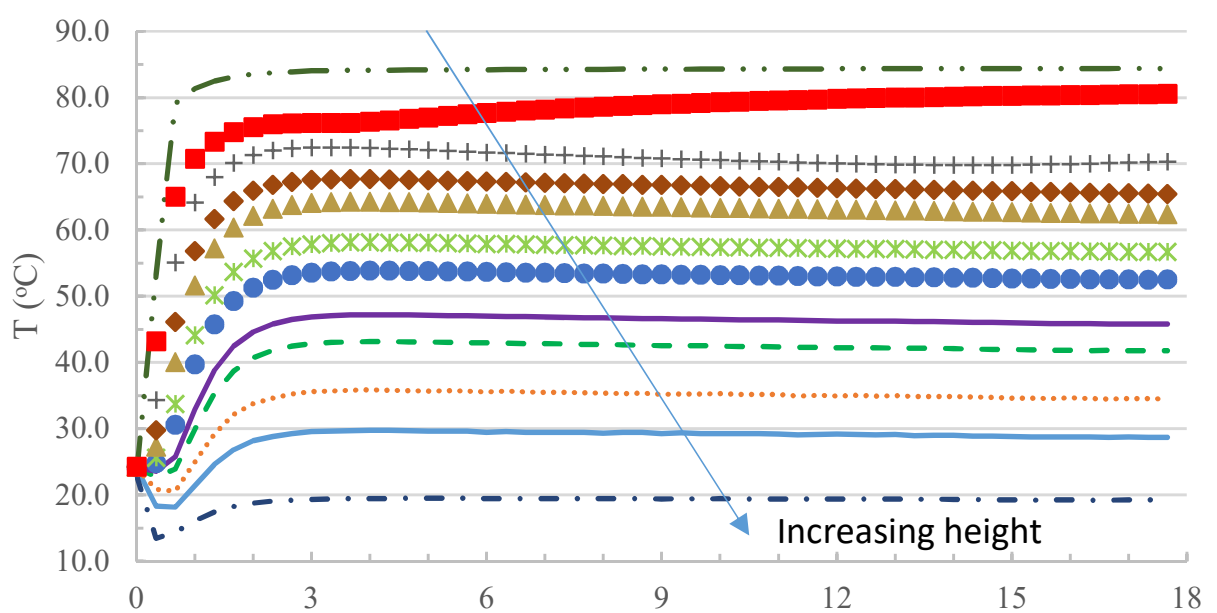

(a)

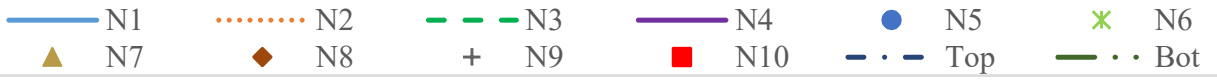

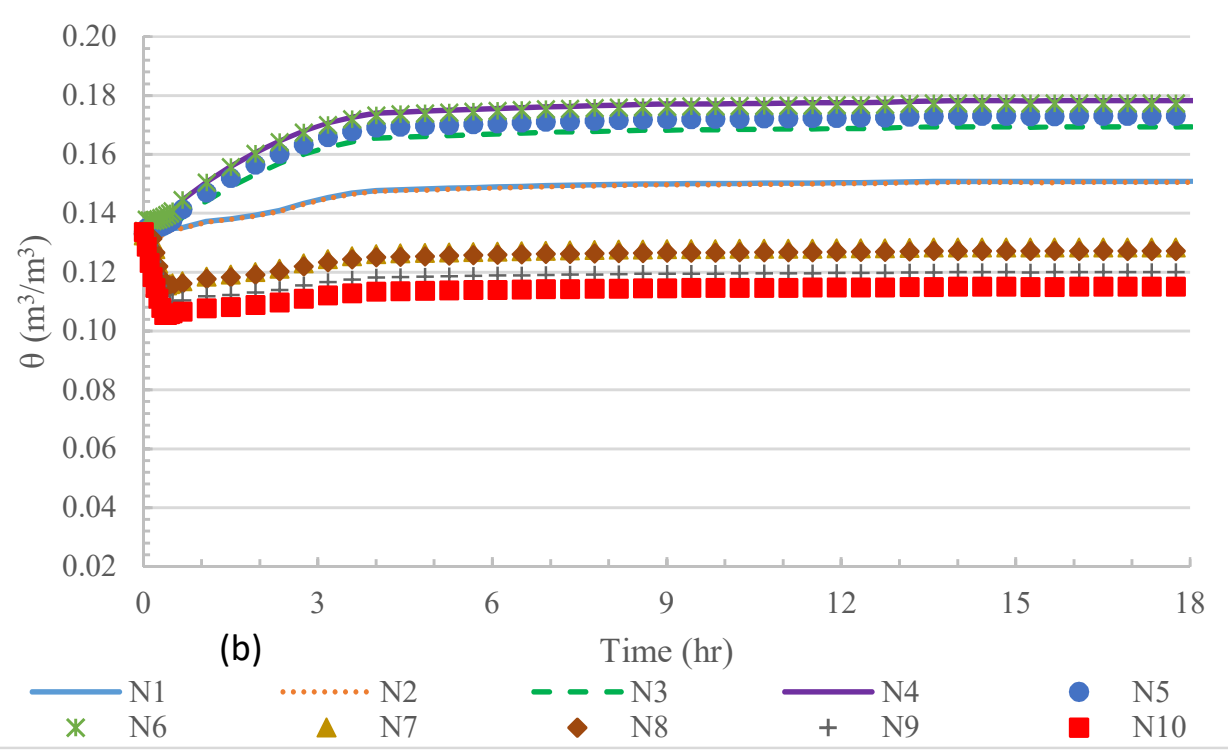

Figure E5. Simulated temperature and moisture content at each needle for the case of wet NB2 $(\eta=0.53, S R=0.25)$ and vertical heating from bottom. (a): temperature response. (b): moisture content response. 

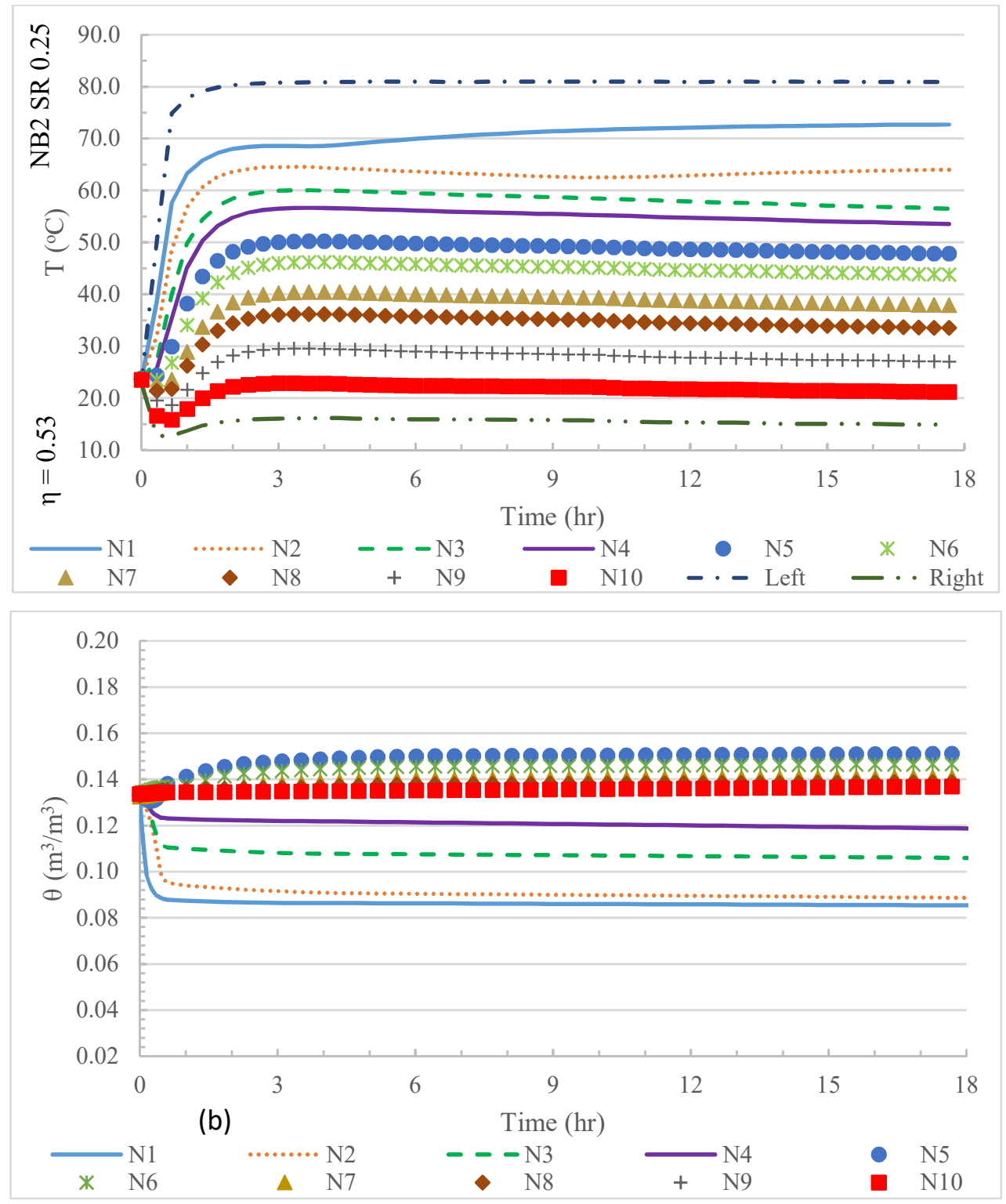

Figure E6. Simulated temperature and moisture content at each needle for the case of wet $N B 2(\eta=0.53, S R=0.25)$ and horizontal heating. (a): temperature response. (b): moisture content response. 


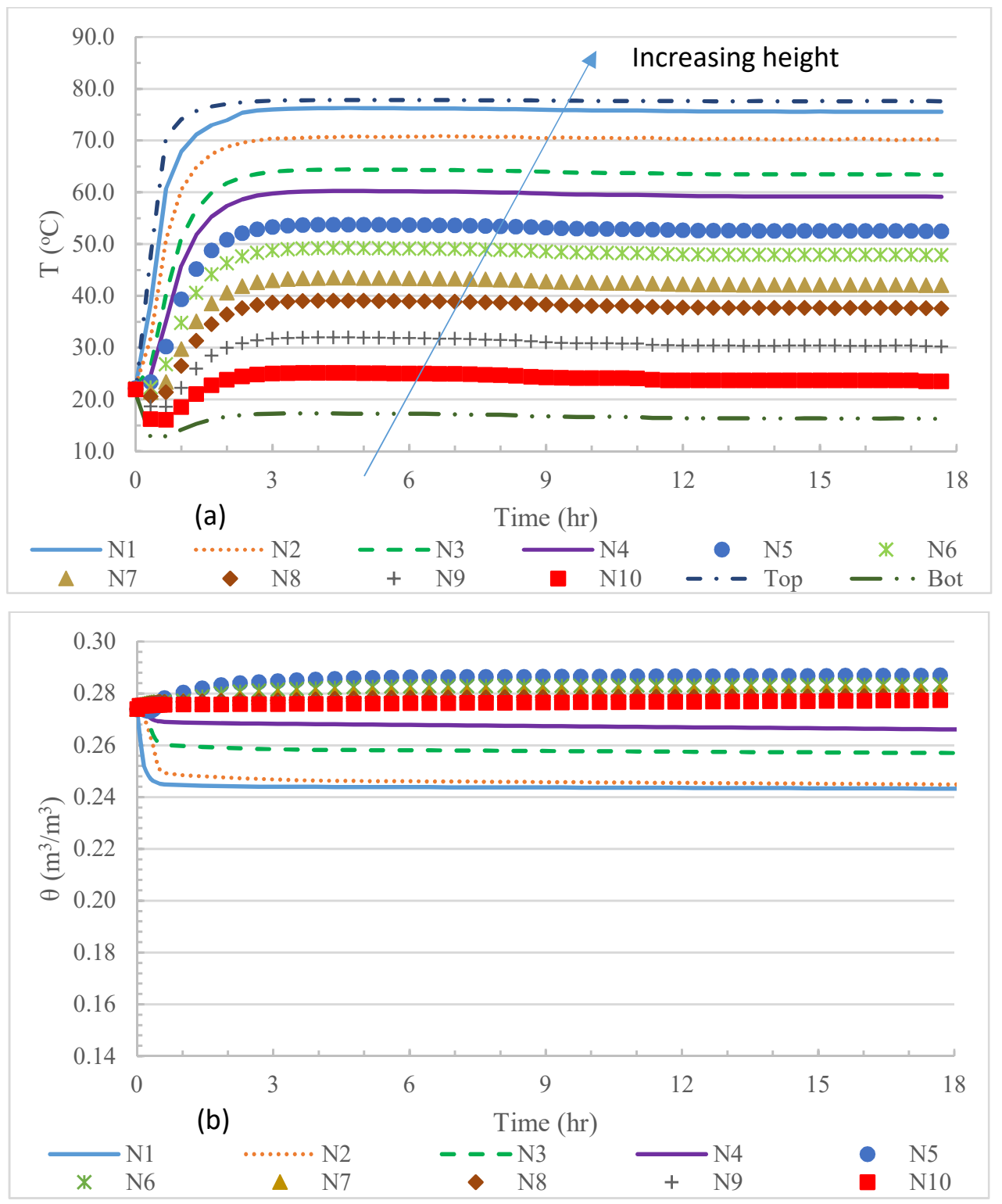

Figure E7. Simulated temperature and moisture content at each needle for the case of wet NB2 $(\eta=0.55, S R=0.50)$ and vertical heating from top. $(a)$ : temperature response. $(b)$ : moisture content response. 


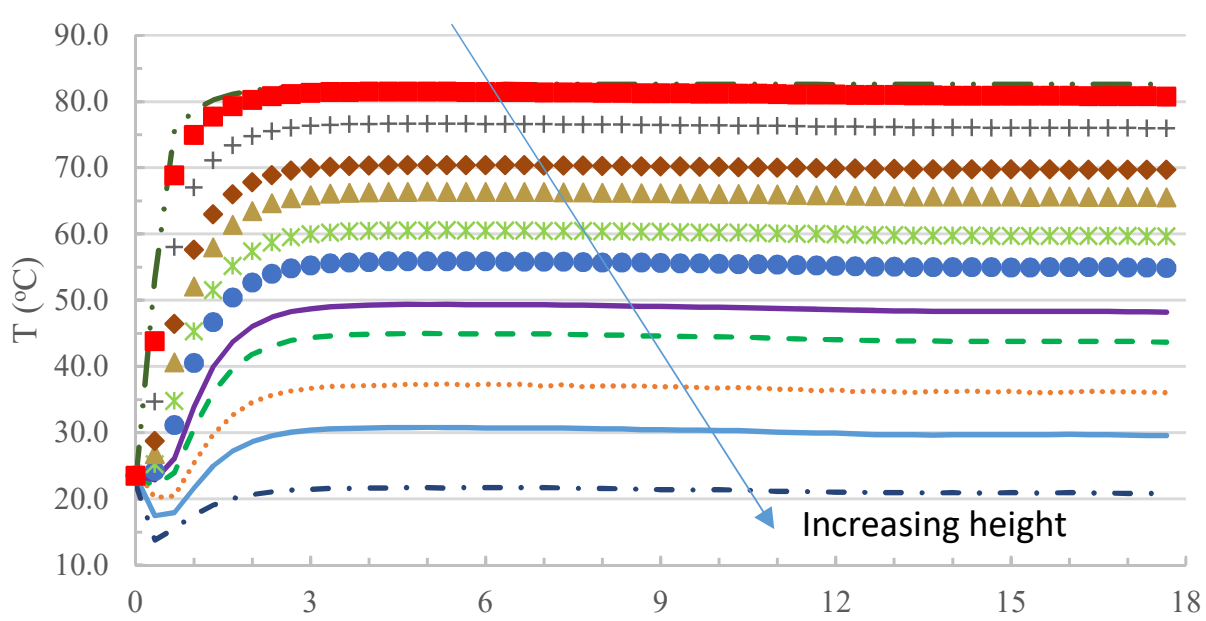

(a)

Time (hr)
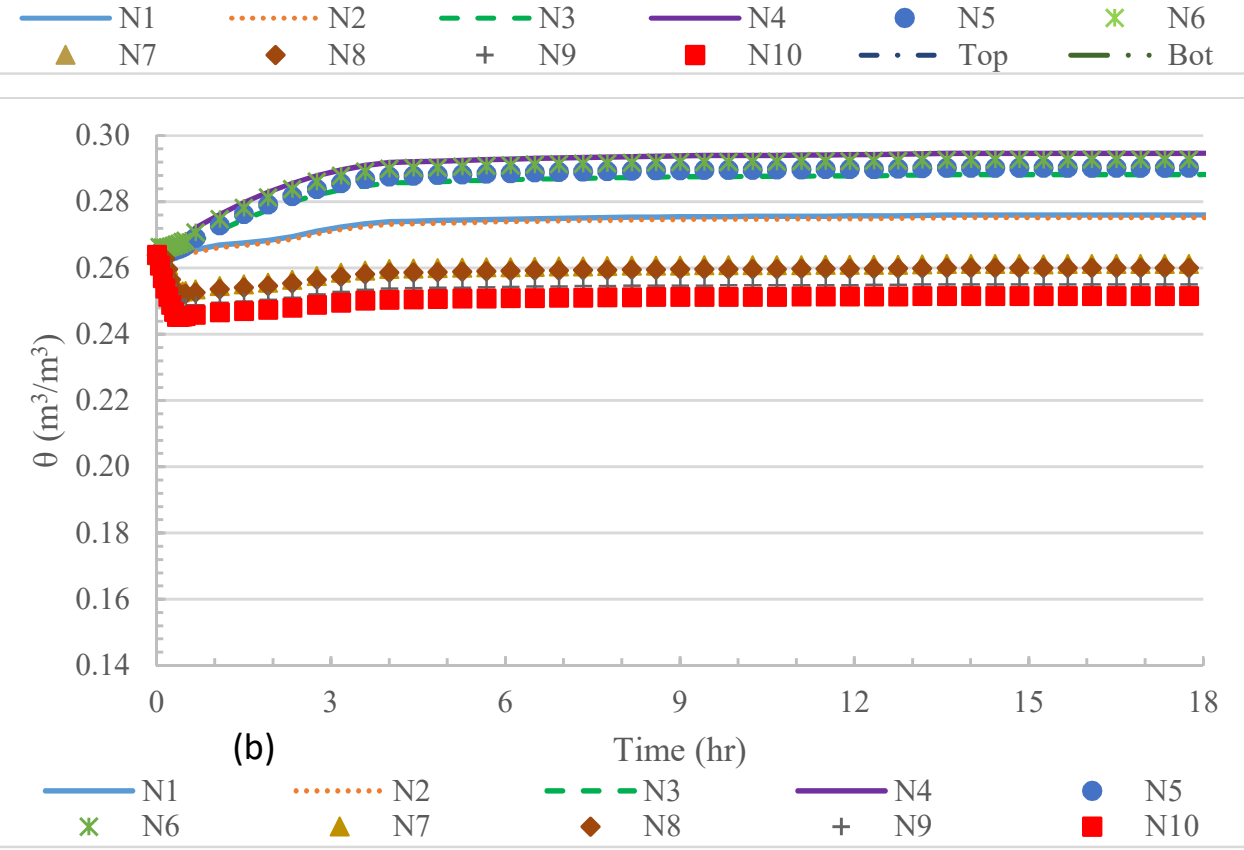

Figure E8. Simulated temperature and moisture content at each needle for the case of wet NB2 $(\eta=0.53, S R=0.50)$ and vertical heating from bottom. $(a)$ : temperature response. $(b)$ : moisture content response. 

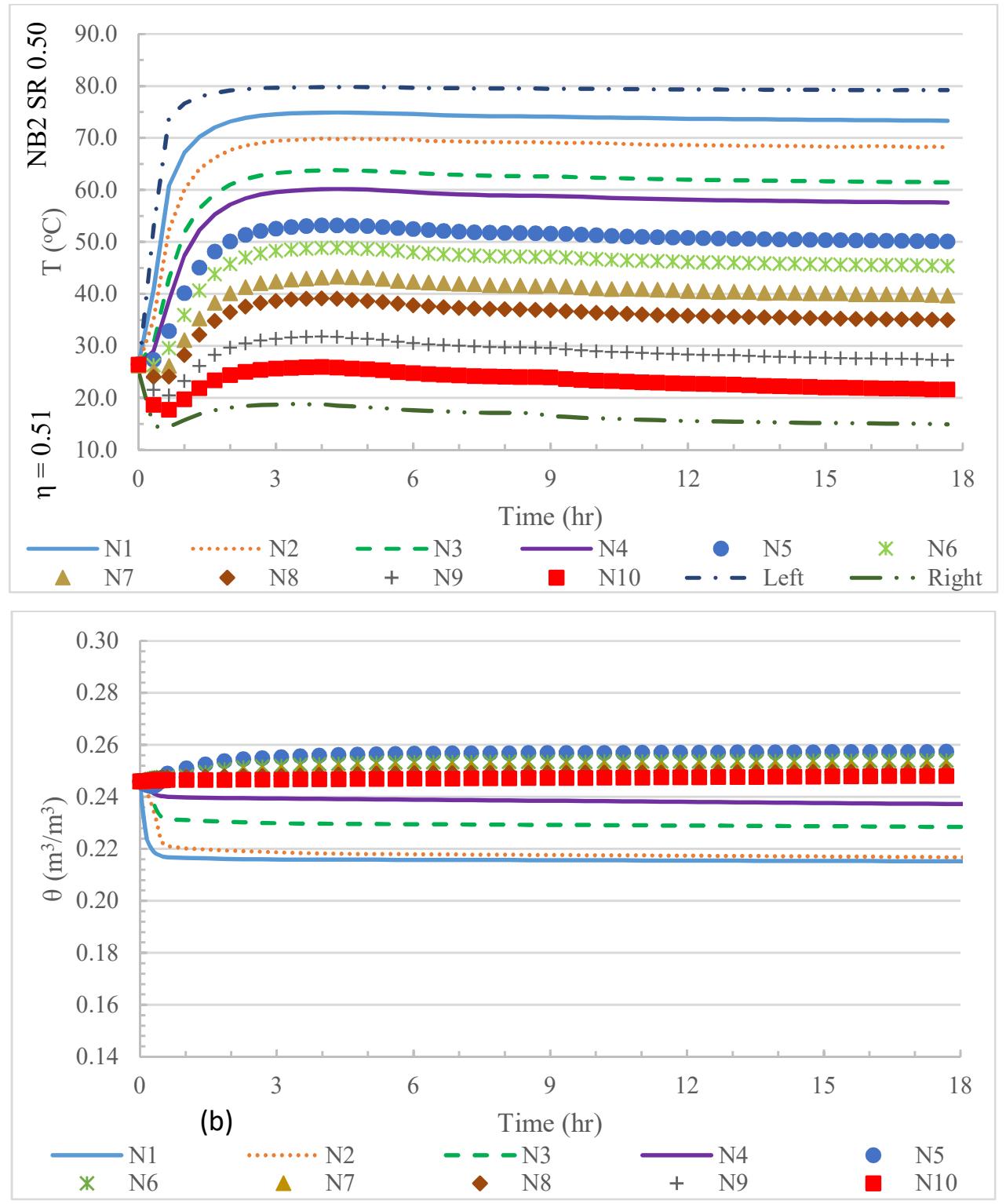

Figure E9. Simulated temperature and moisture content at each needle for the case of wet $N B 2(\eta=0.51, S R=0.50)$ and horizontal heating. (a): temperature response. (b): moisture content response. 


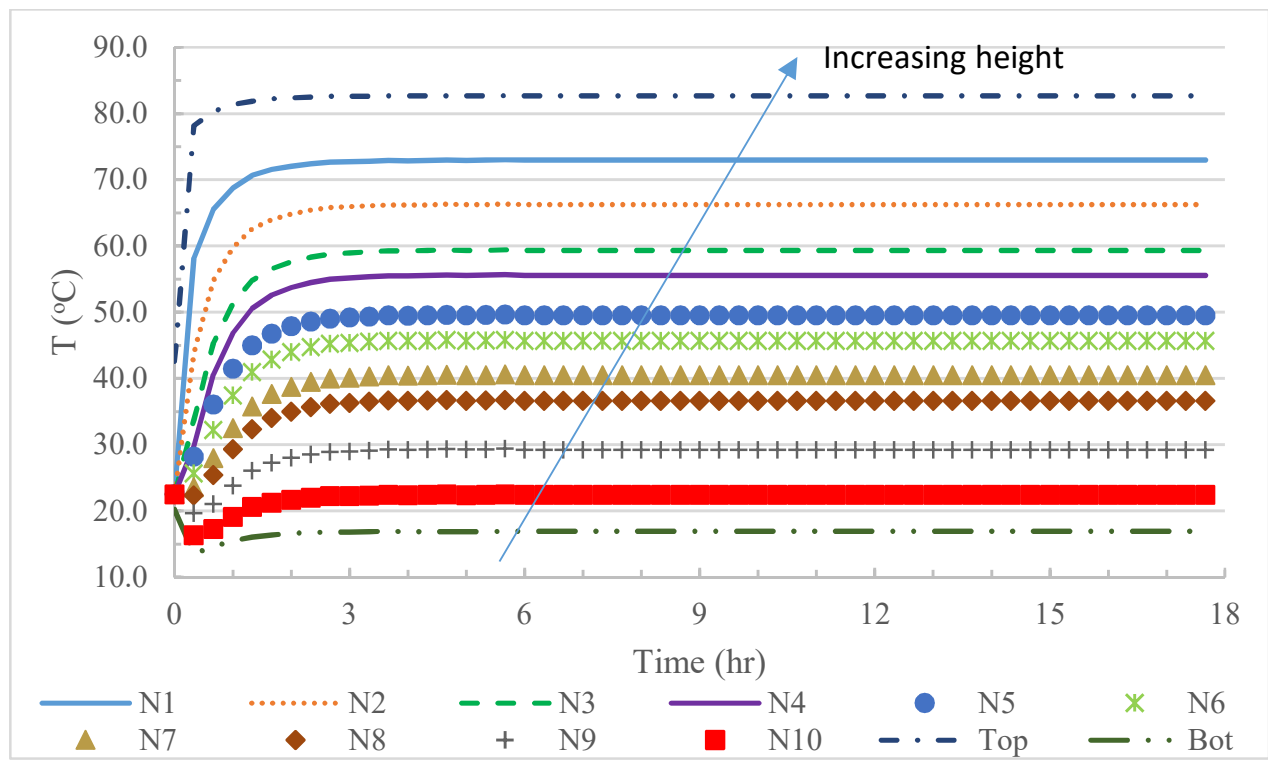

Figure E10. Simulated temperature at each needle (N\#) for the case of dry $Q C 2(\eta=0.45)$ and vertical heating from top.

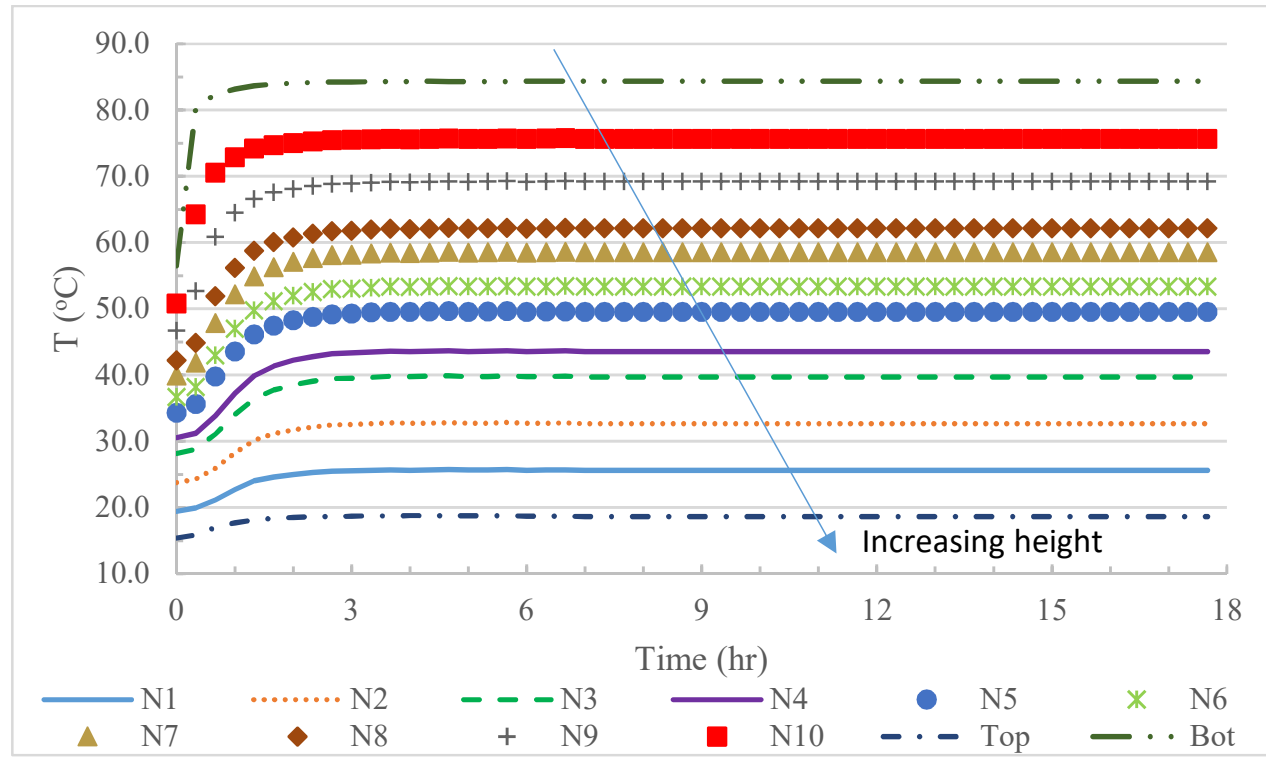

Figure E11. Simulated temperature at each needle (N\#) for the case of dry $Q C 2(\eta=0.45)$ and vertical heating from bottom. 


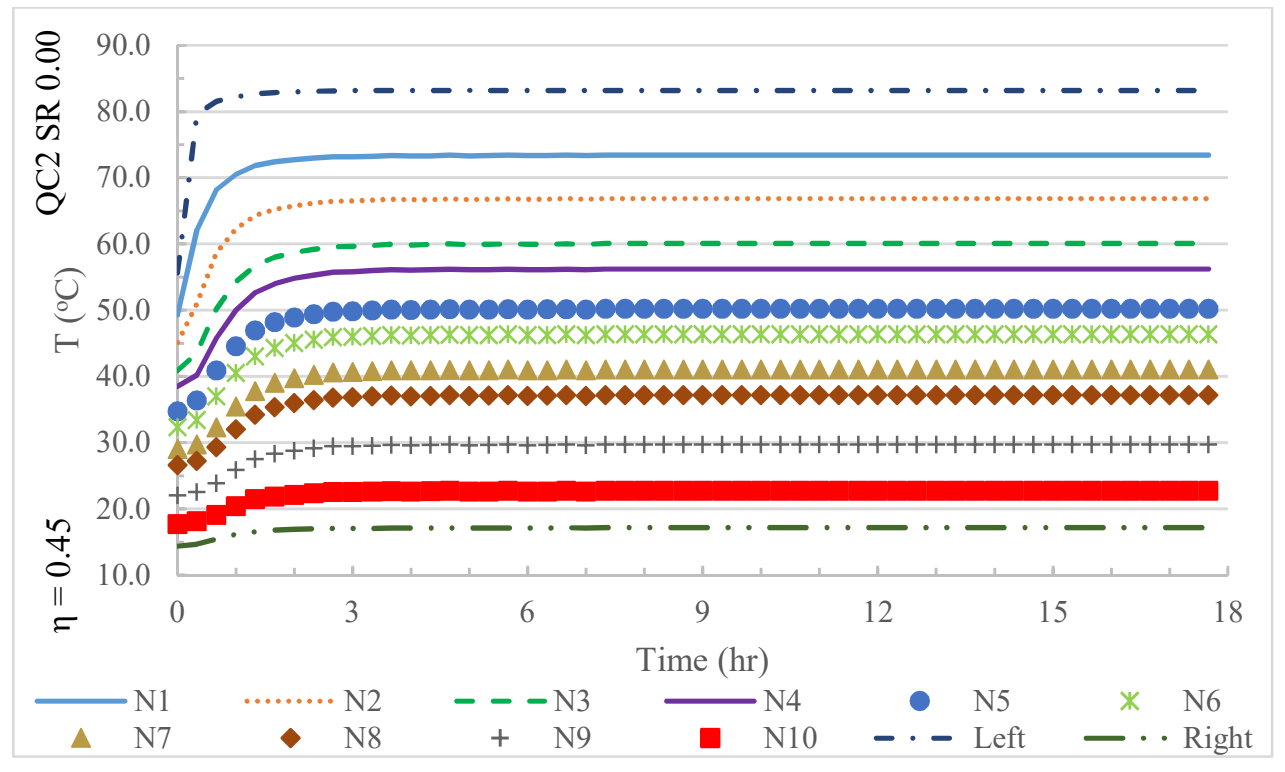

Figure E12. Simulated temperature at each needle (N\#) for the case of dry $Q C 2(\eta=0.45)$ and horizontal heating. 


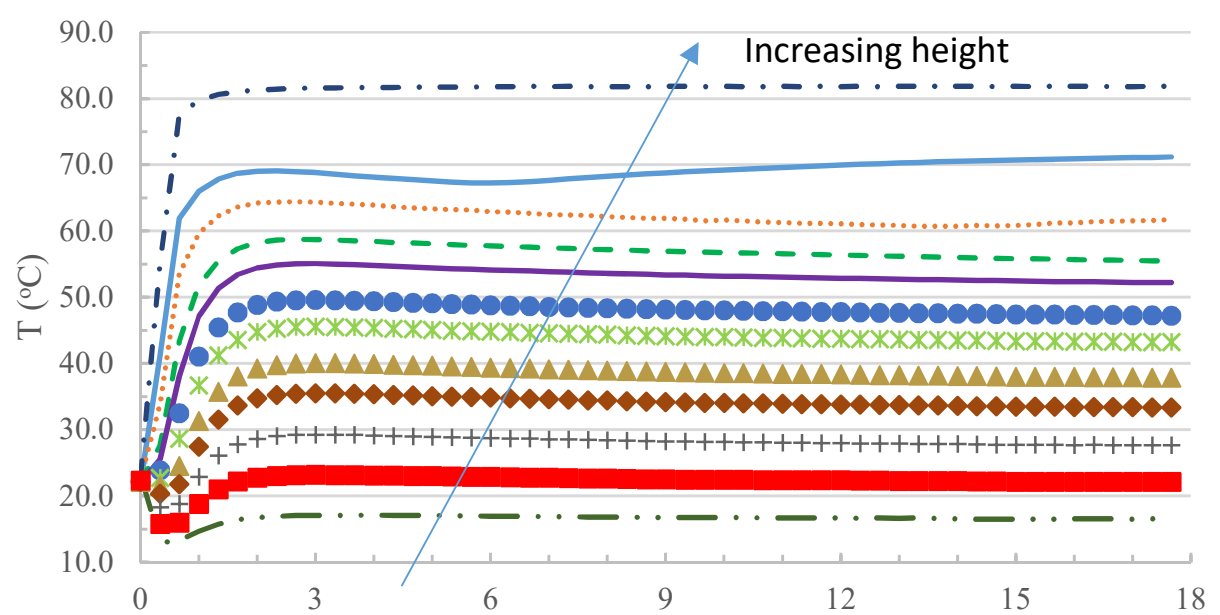

(a)

Time (hr)

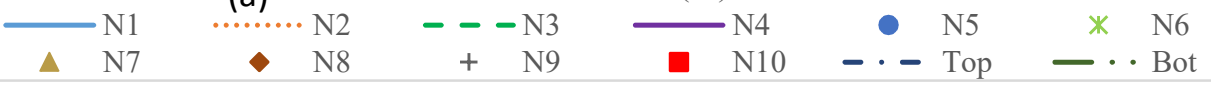

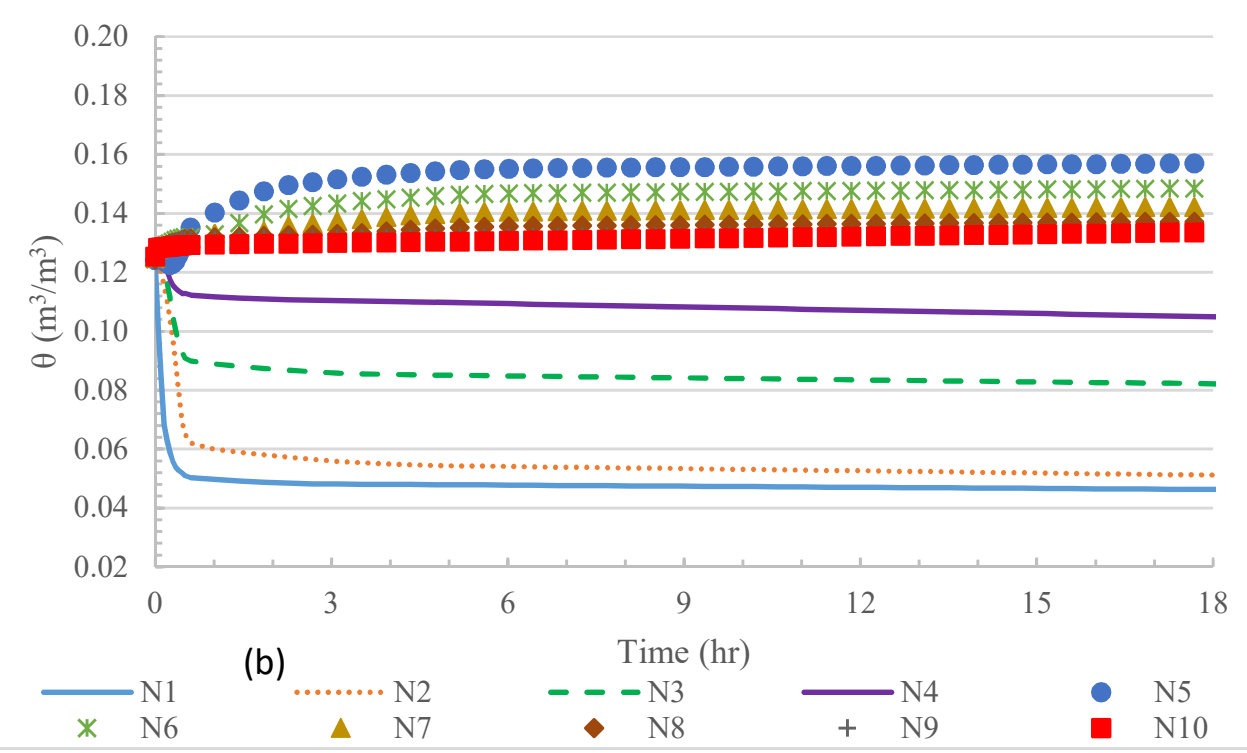

Figure E13. Simulated temperature and moisture content at each needle for the case of wet QC2 $(\eta=0.50, S R=0.25)$ and vertical heating from top. $(a)$ : temperature response. $(b)$ : moisture content response. 


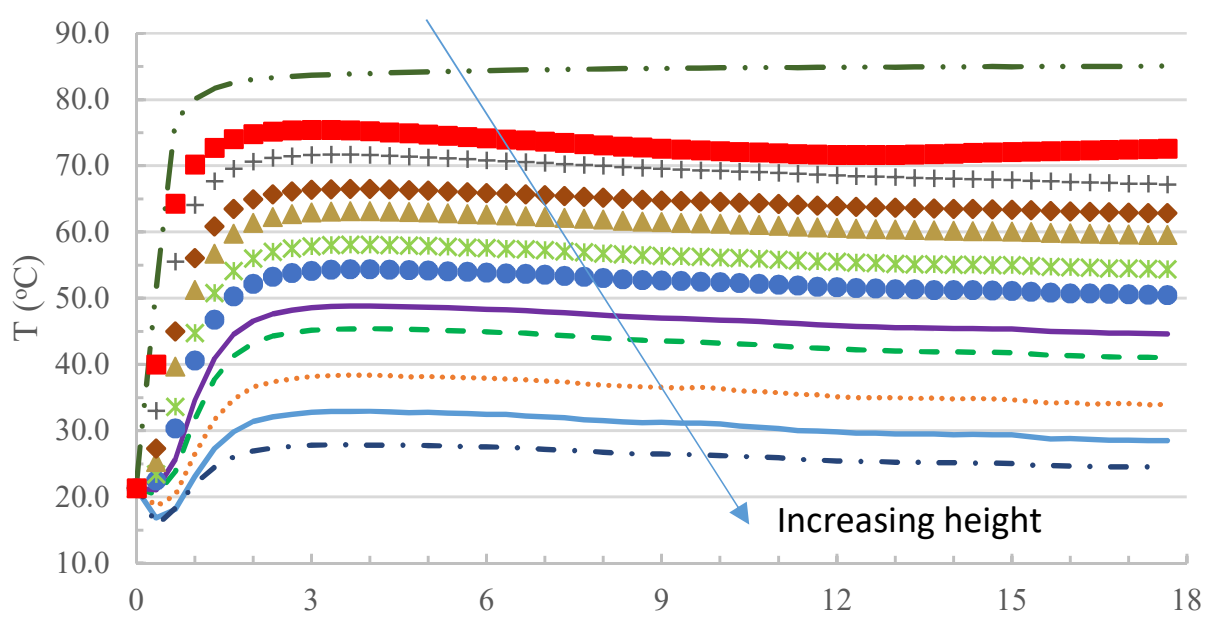

(a)

Time (hr)

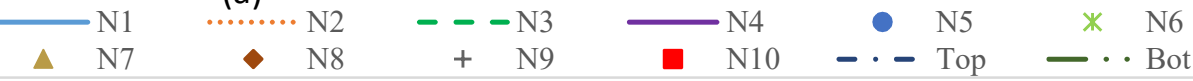

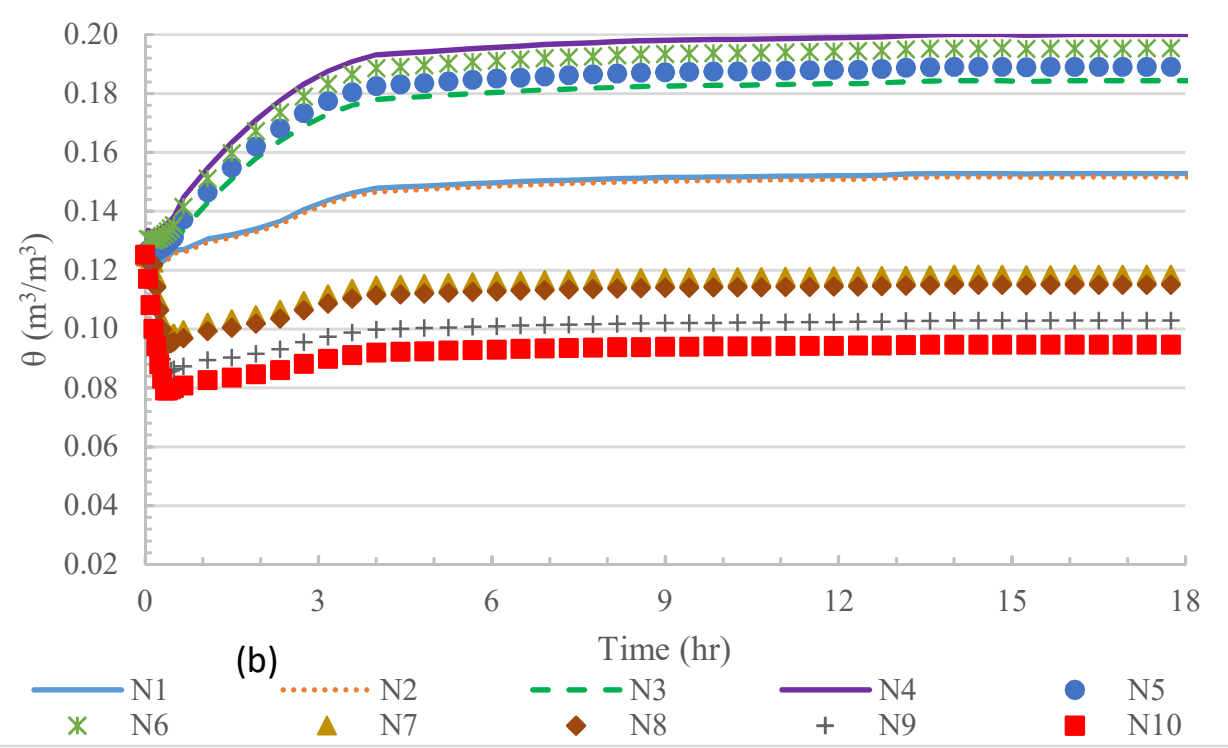

Figure E14. Simulated temperature and moisture content at each needle for the case of wet QC2 $(\eta=0.48, S R=0.25)$ and vertical heating from bottom. (a): temperature response. (b): moisture content response. 

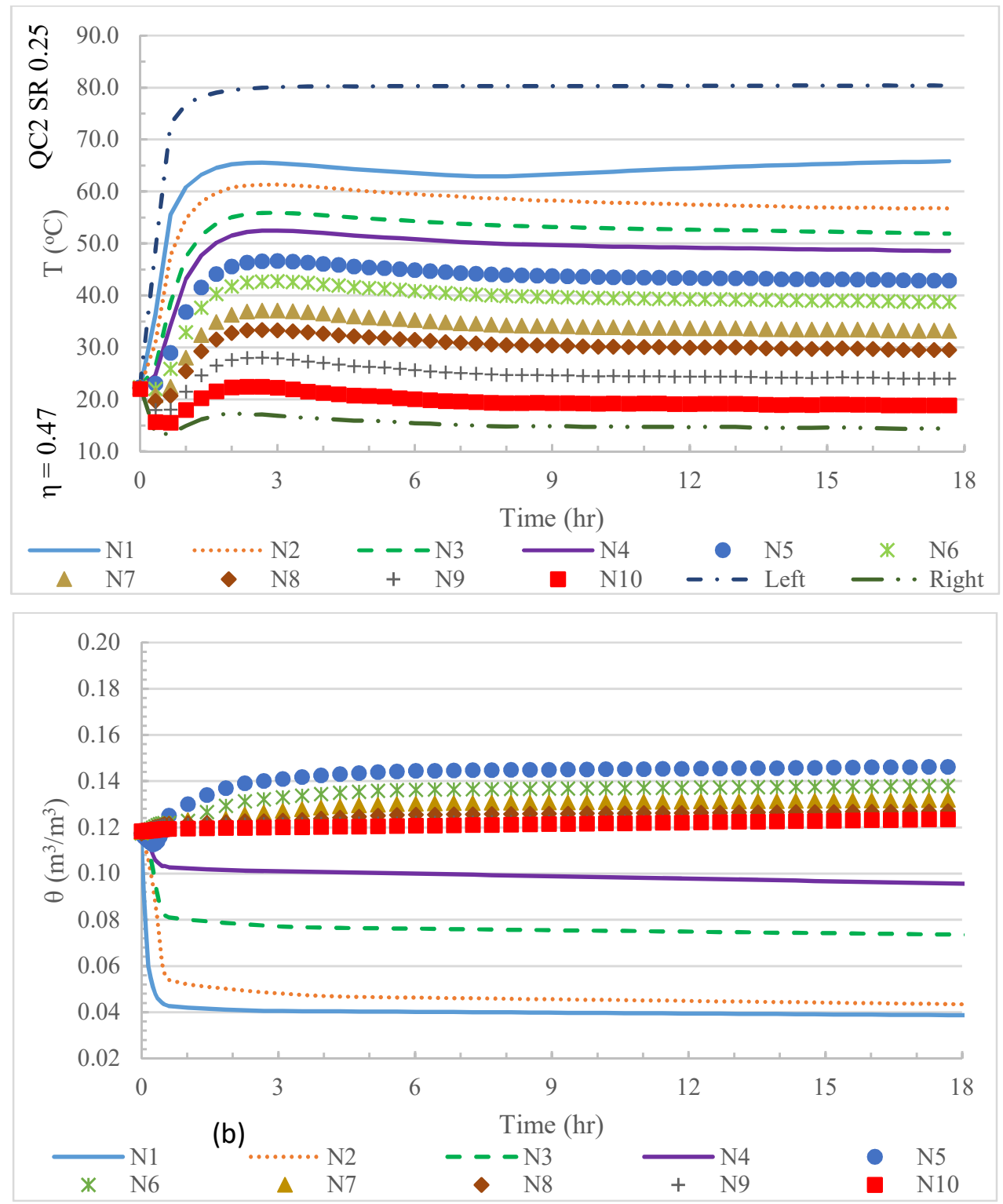

Figure E15. Simulated temperature and moisture content at each needle for the case of wet QC2 $(\eta=0.47, S R=0.25)$ and horizontal heating. (a): temperature response. (b): moisture content response. 


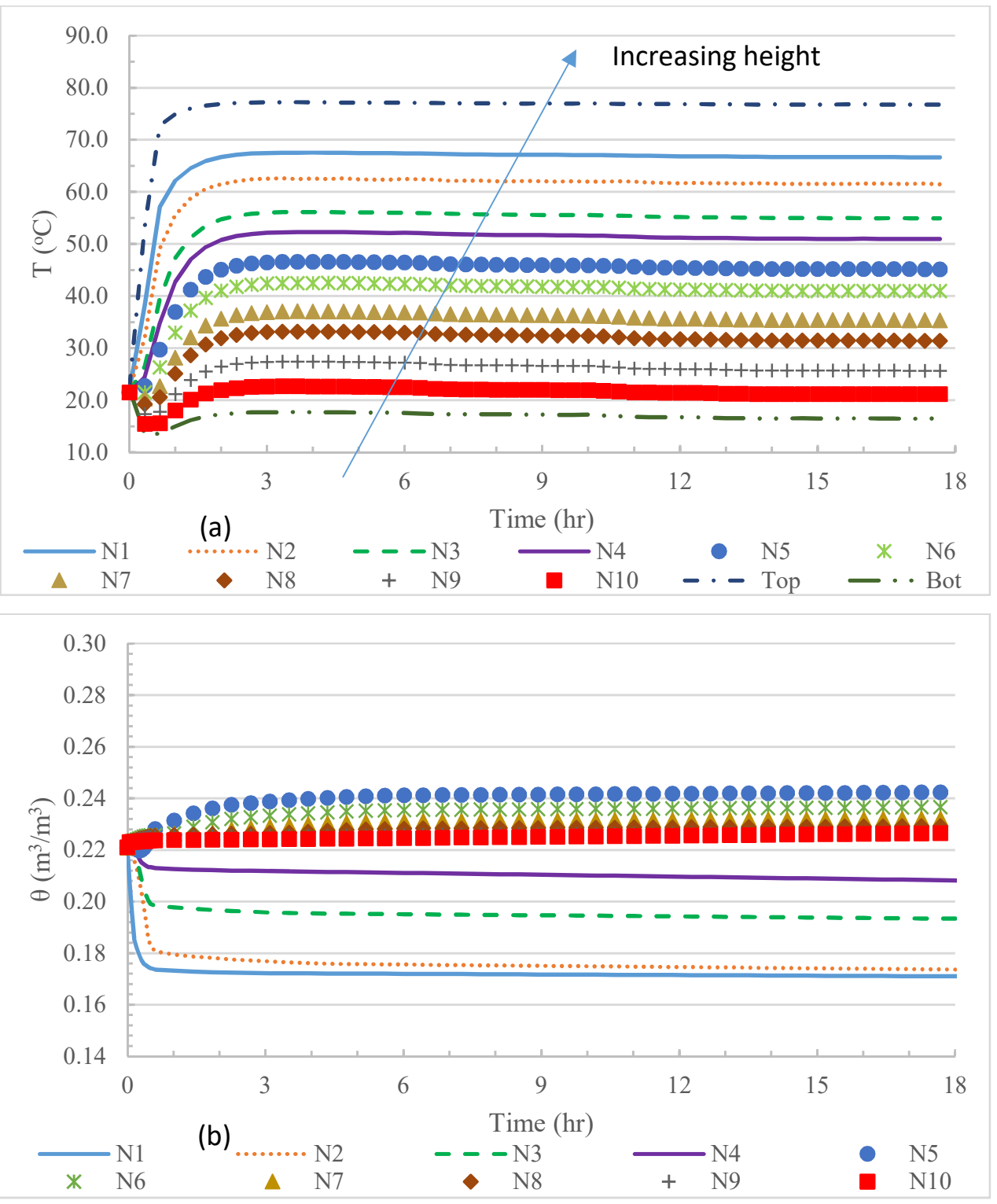

Figure E16. Simulated temperature and moisture content at each needle for the case of wet $Q C 2(\eta=0.44, S R=0.50)$ and vertical heating from top. $(a)$ : temperature response. $(b)$ : moisture content response. 


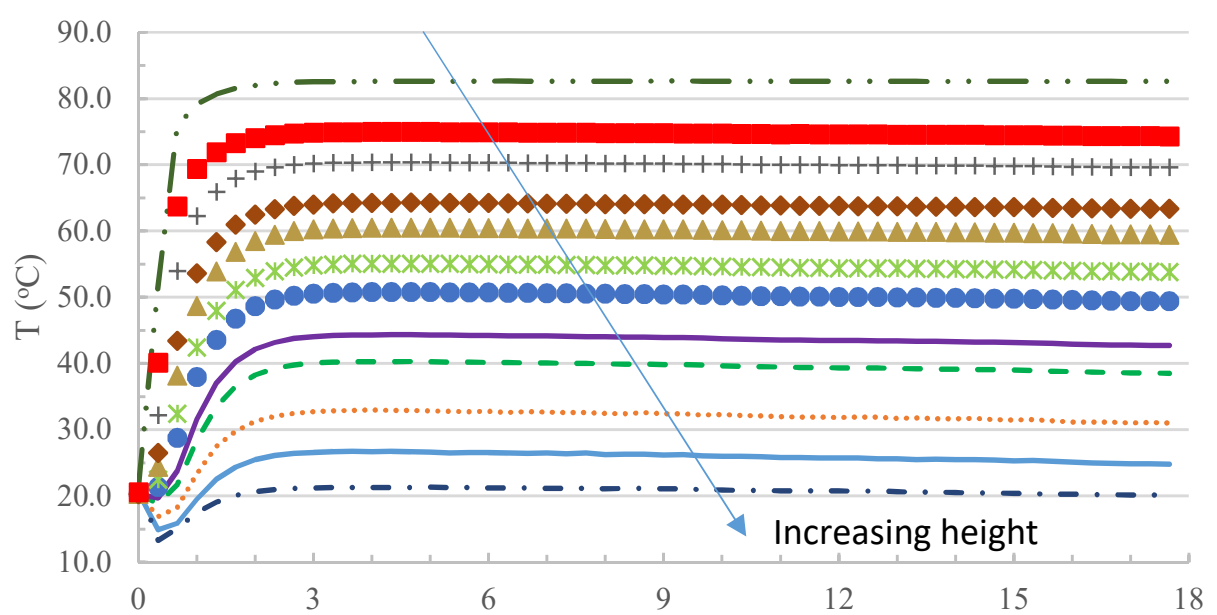
(a)
Time (hr)

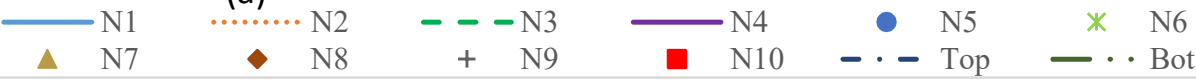

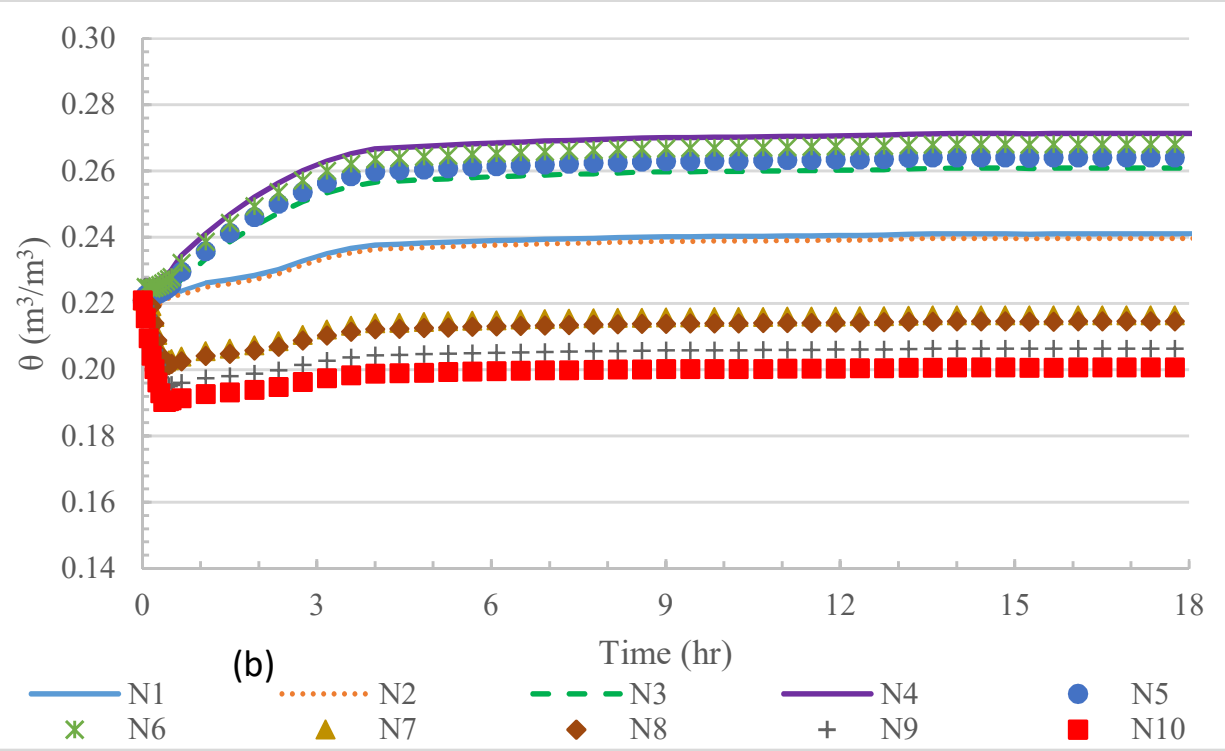

Figure E17. Simulated temperature and moisture content at each needle for the case of wet QC2 $(\eta=0.44, S R=0.50)$ and vertical heating from bottom. (a): temperature response. (b): moisture content response. 

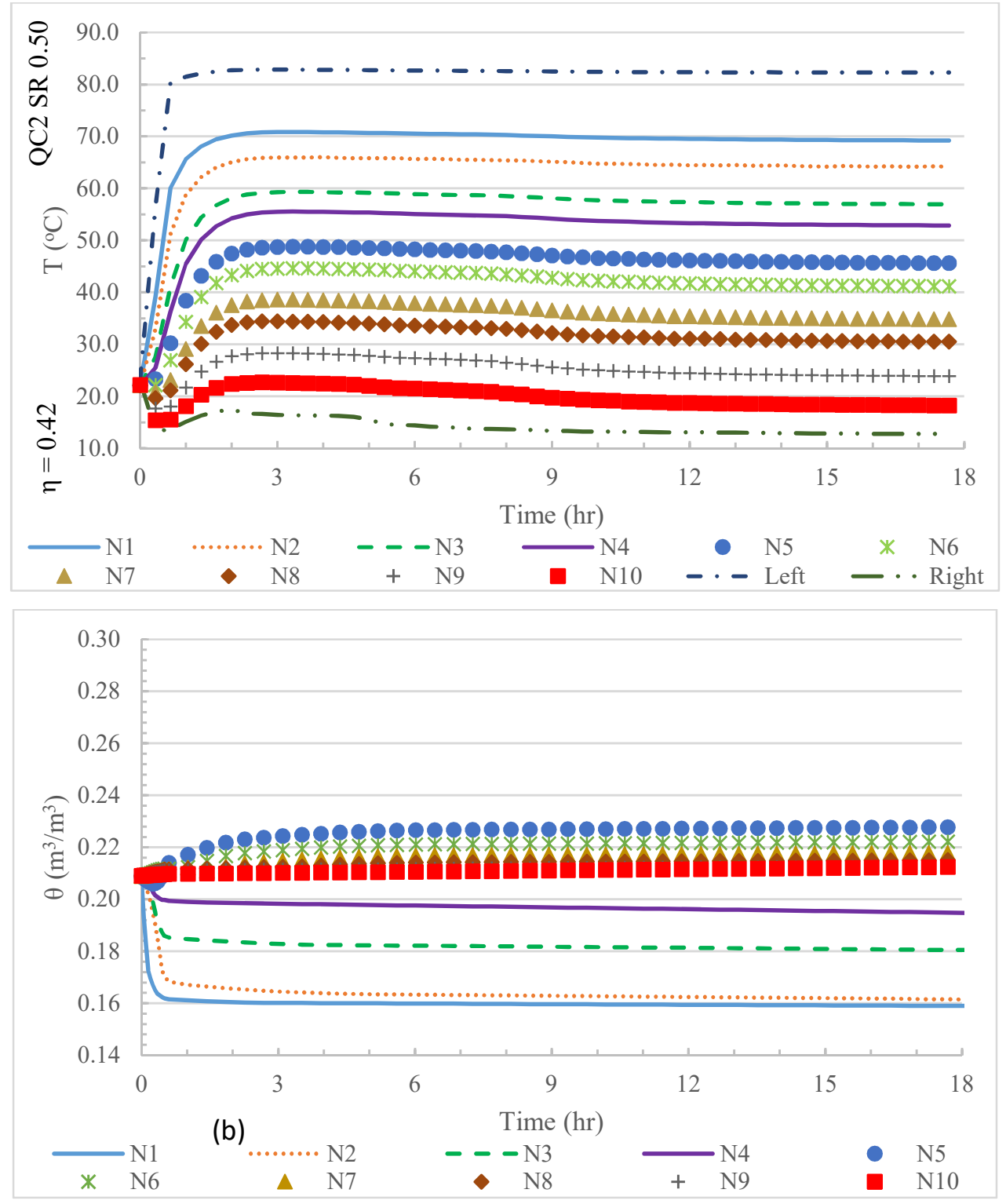

Figure E18. Simulated temperature and moisture content at each needle for the case of wet QC2 $(\eta=0.42, S R=0.50)$ and horizontal heating. (a): temperature response. (b): moisture content response. 

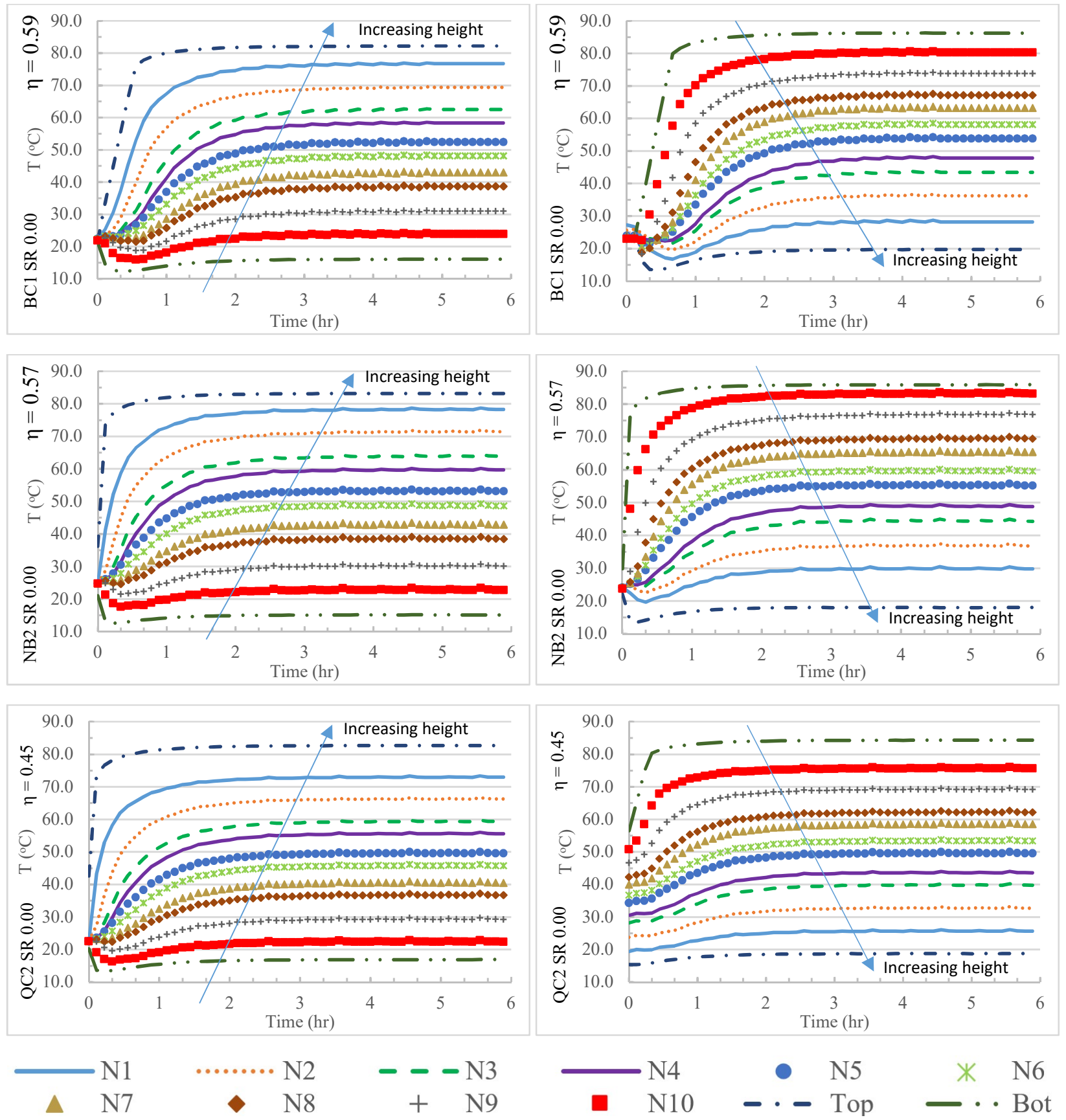

$\begin{array}{rrrrrr} & \mathrm{N} 1 & \ldots \ldots & \mathrm{N} 2 & - & -\mathrm{N} 3 \\ & \mathrm{~N} 7 & \bullet & \mathrm{N} 8 & + & \mathrm{N} 9\end{array}$

Figure E19. Simulated temperature at each needle (N\#) for the case of dry soils and vertical heating from top (left part) and from bottom (right part) from 0 to 6 hour. 

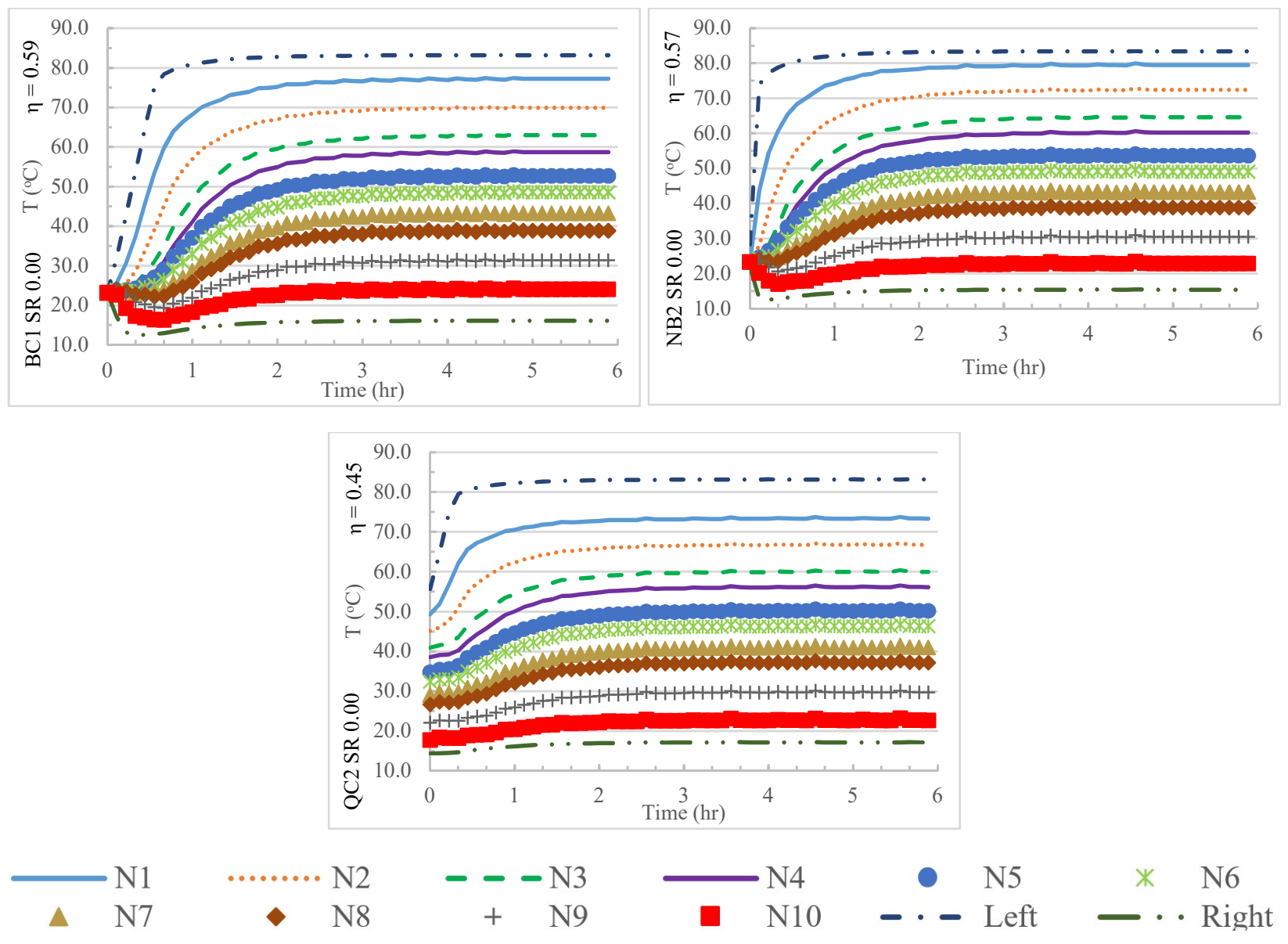

Figure E20. Simulated temperature at each needle (N\#) for the case of dry soils and horizontal heating from 0 to 6 hour. 

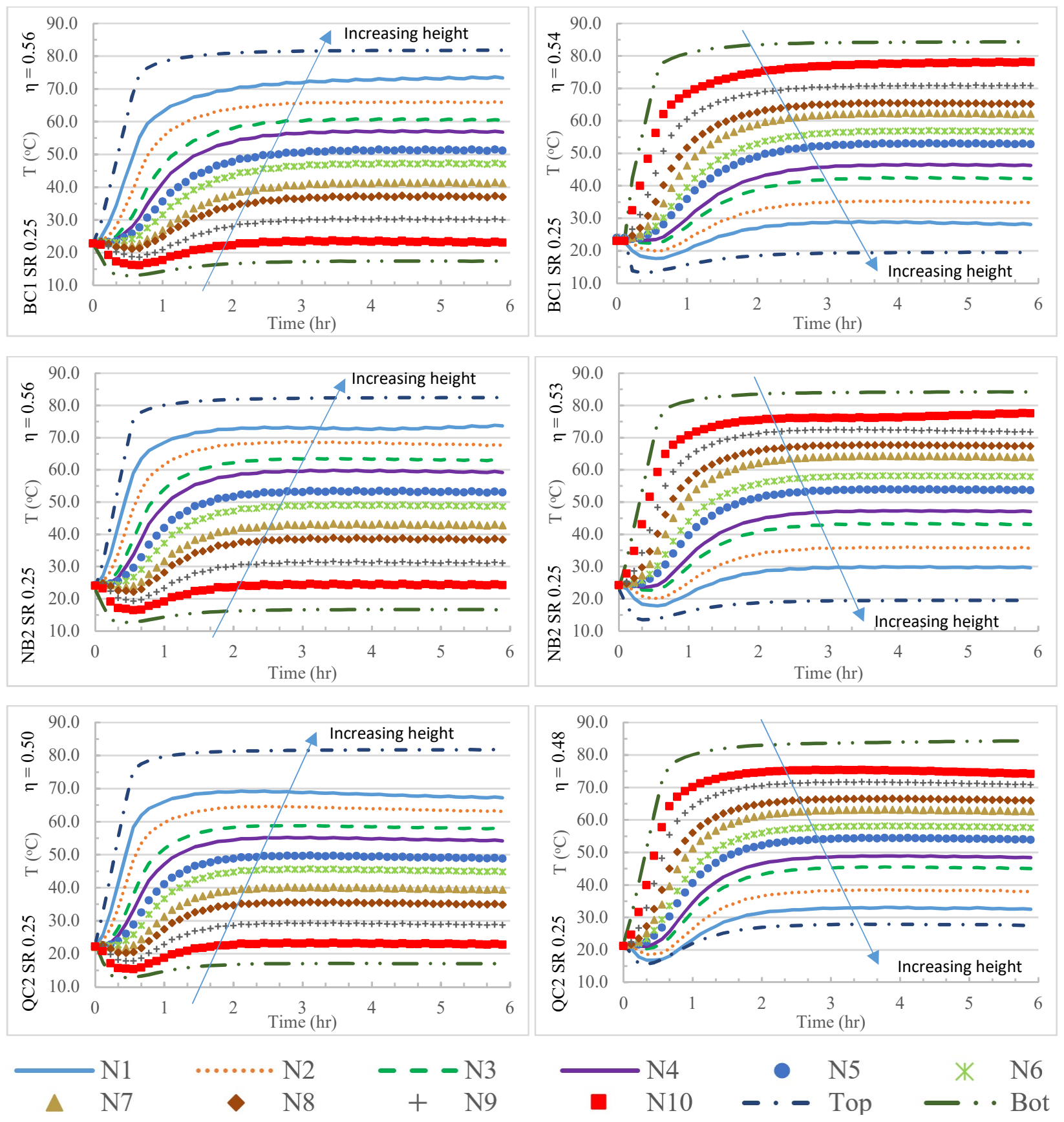

Figure E21. Simulated temperature at each needle (N\#) for the case of wet soils (SR $\sim 0.25)$ and vertical heating from top (left part) and from bottom (right part) from 0 to 6 hour. 

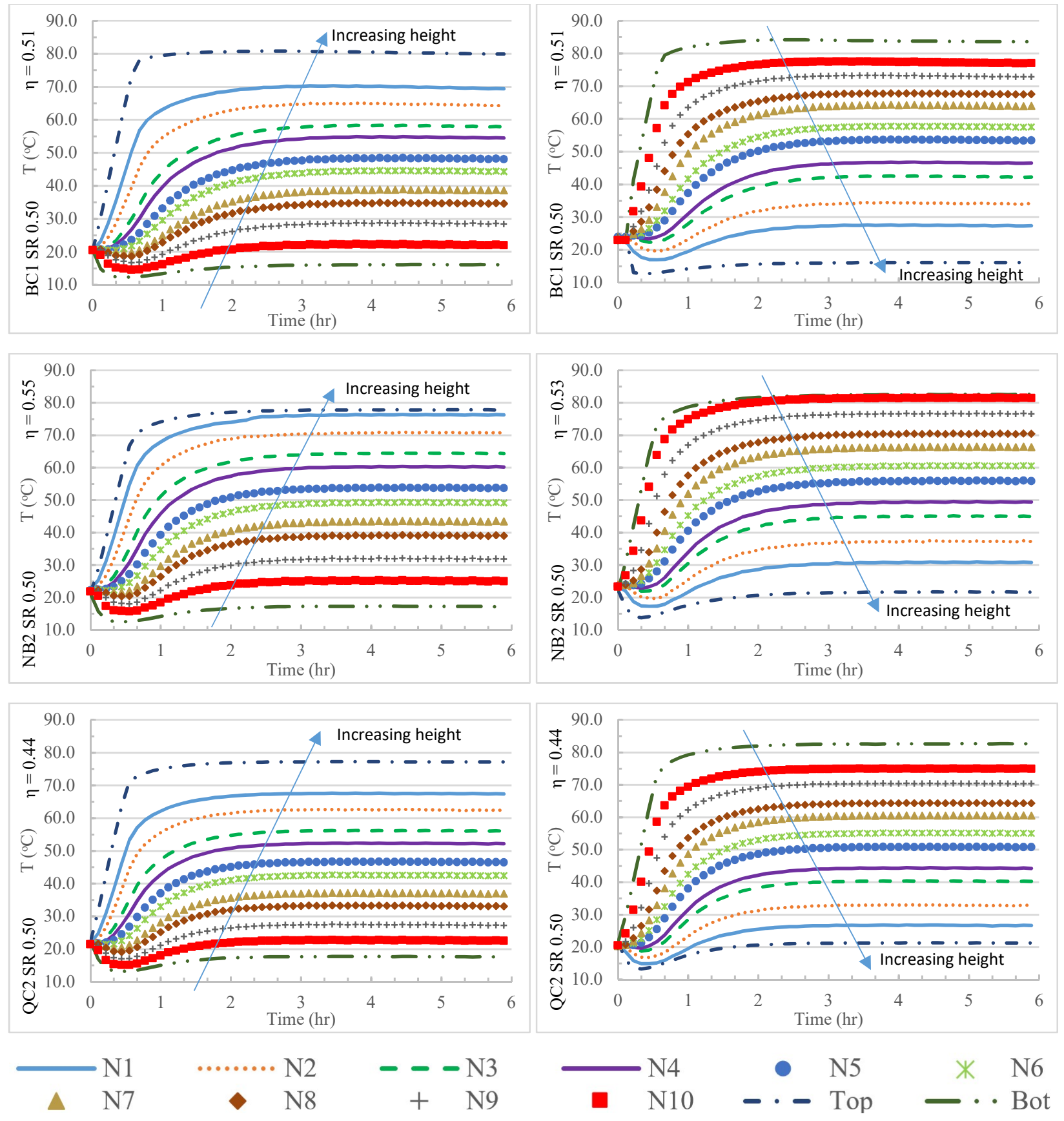

Figure E22. Simulated temperature at each needle (N\#) for the case of wet soils $(S R \sim 0.50)$ and vertical heating from top (left part) and from bottom (right part) from 0 to 6 hour. 

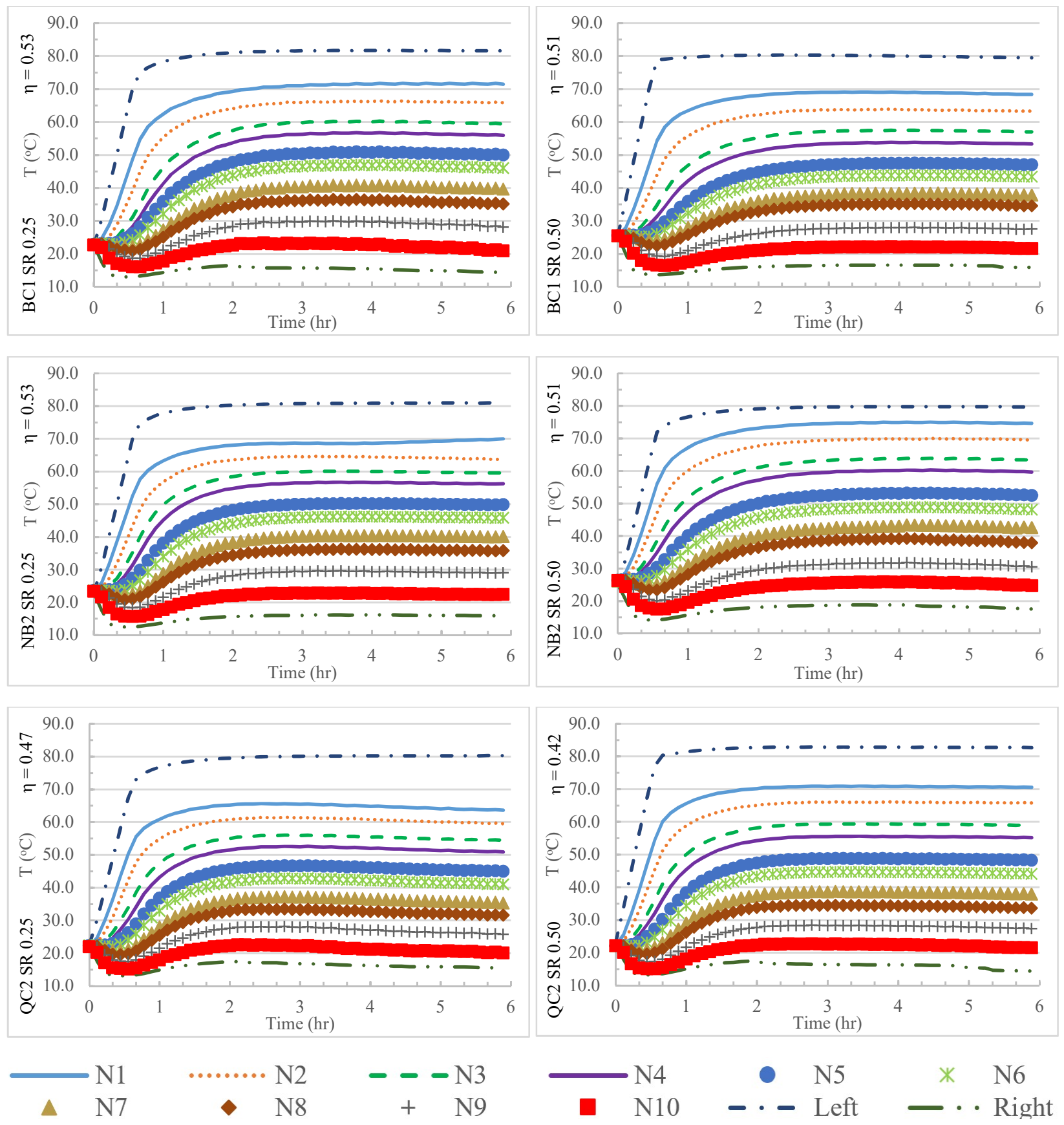

Figure E23. Simulated temperature at each needle (N\#) for the case of wet soils being horizontally heated from 0 to 6 hour. $S R \approx 0.25$ is on the left. $S R \approx 0.50$ is on the right. 

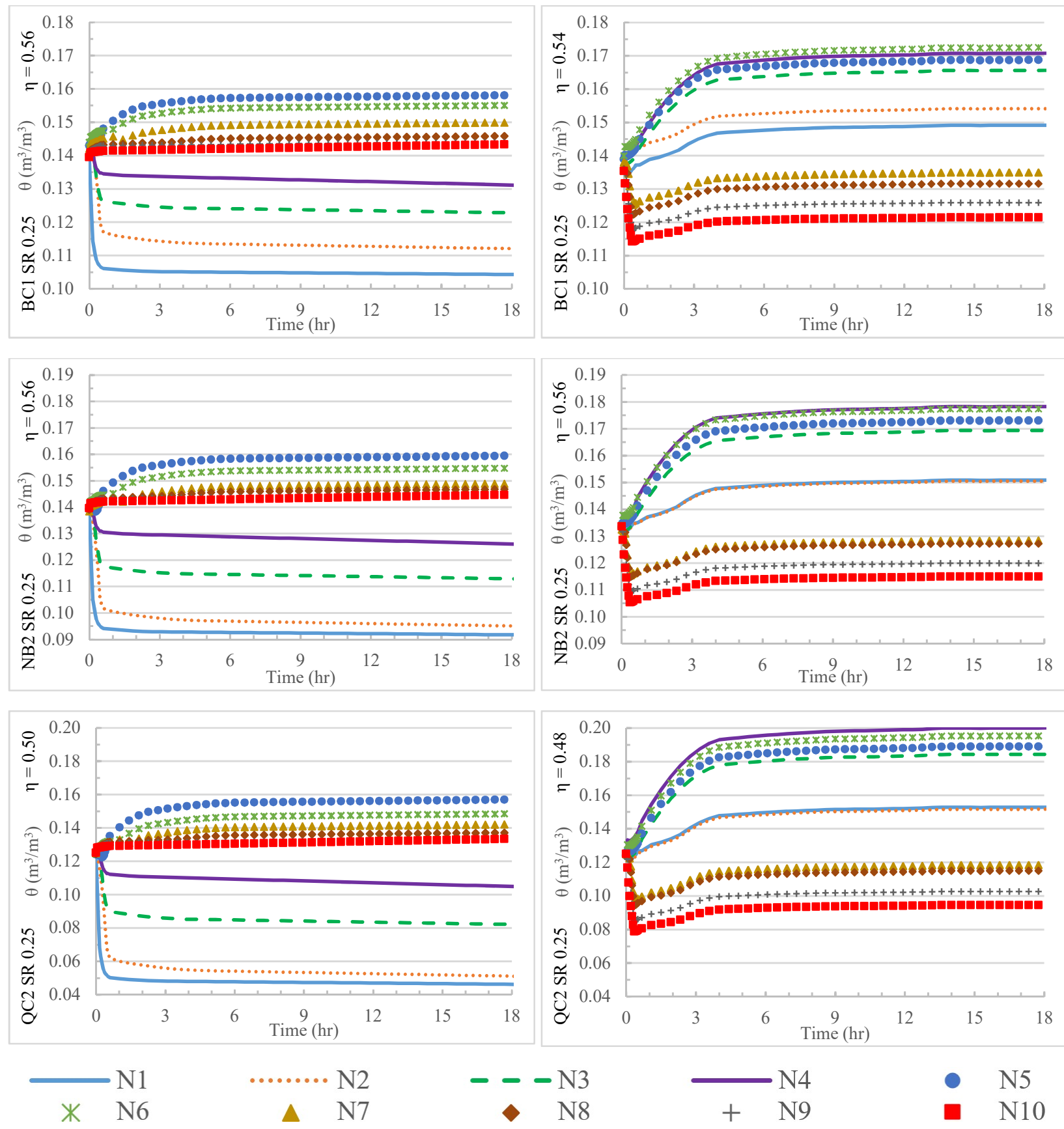

Figure E24. Simulated moisture content at each needle (N\#) for the case of wet soils (SR 0.25) and vertical heating from top (left part) and from bottom (right part) from 0 to 18 hour. 

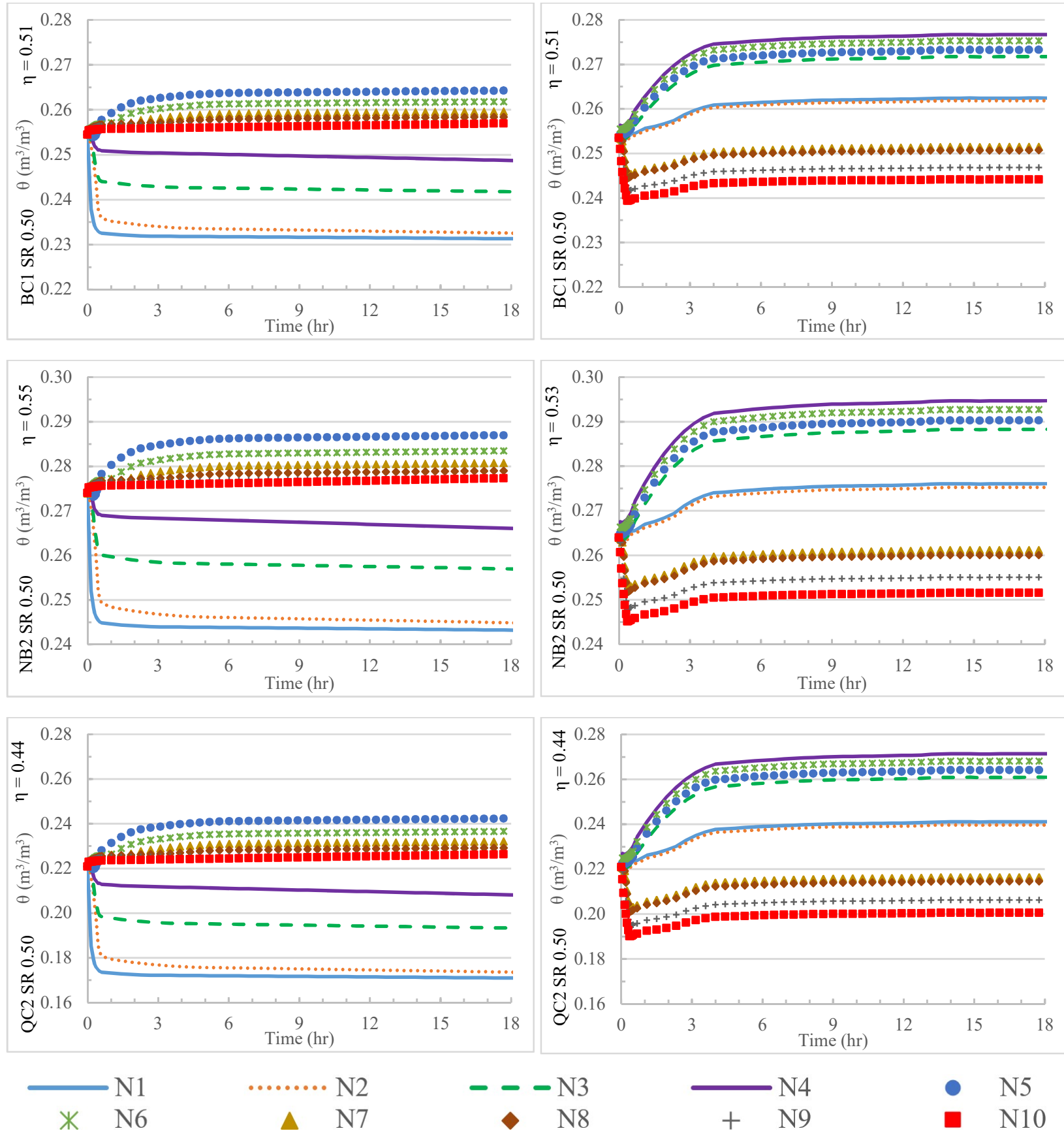

Figure E25. Simulated moisture content at each needle (N\#) for the case of wet soils (SR 0.50) and vertical heating from top (left part) and from bottom (right part) from 0 to 18 hour. 

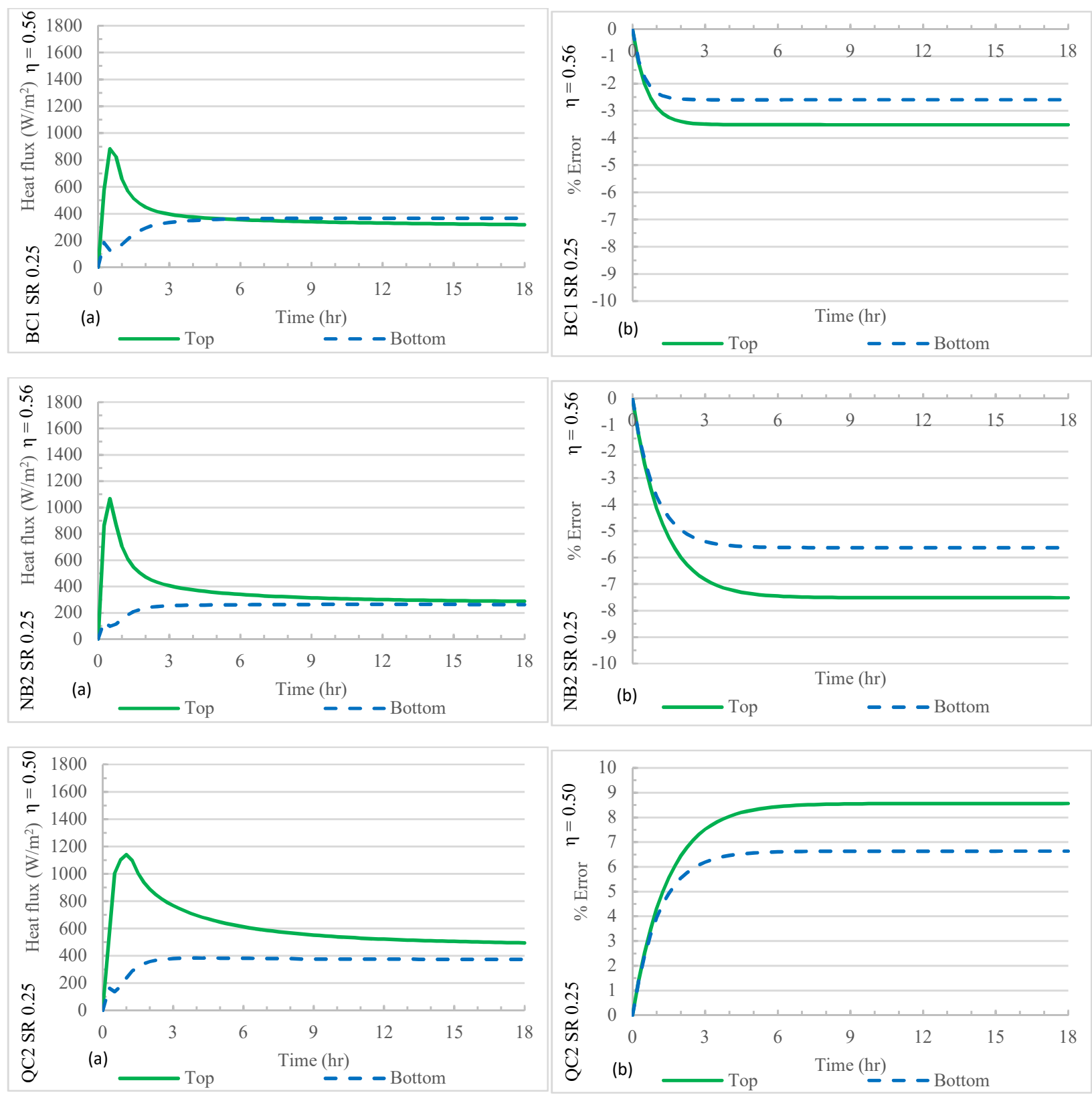

Figure E26. Simulated heat flux and \% error at top and bottom HFMs of wet soils $(S R \approx 0.25)$ and vertical heating from top. 

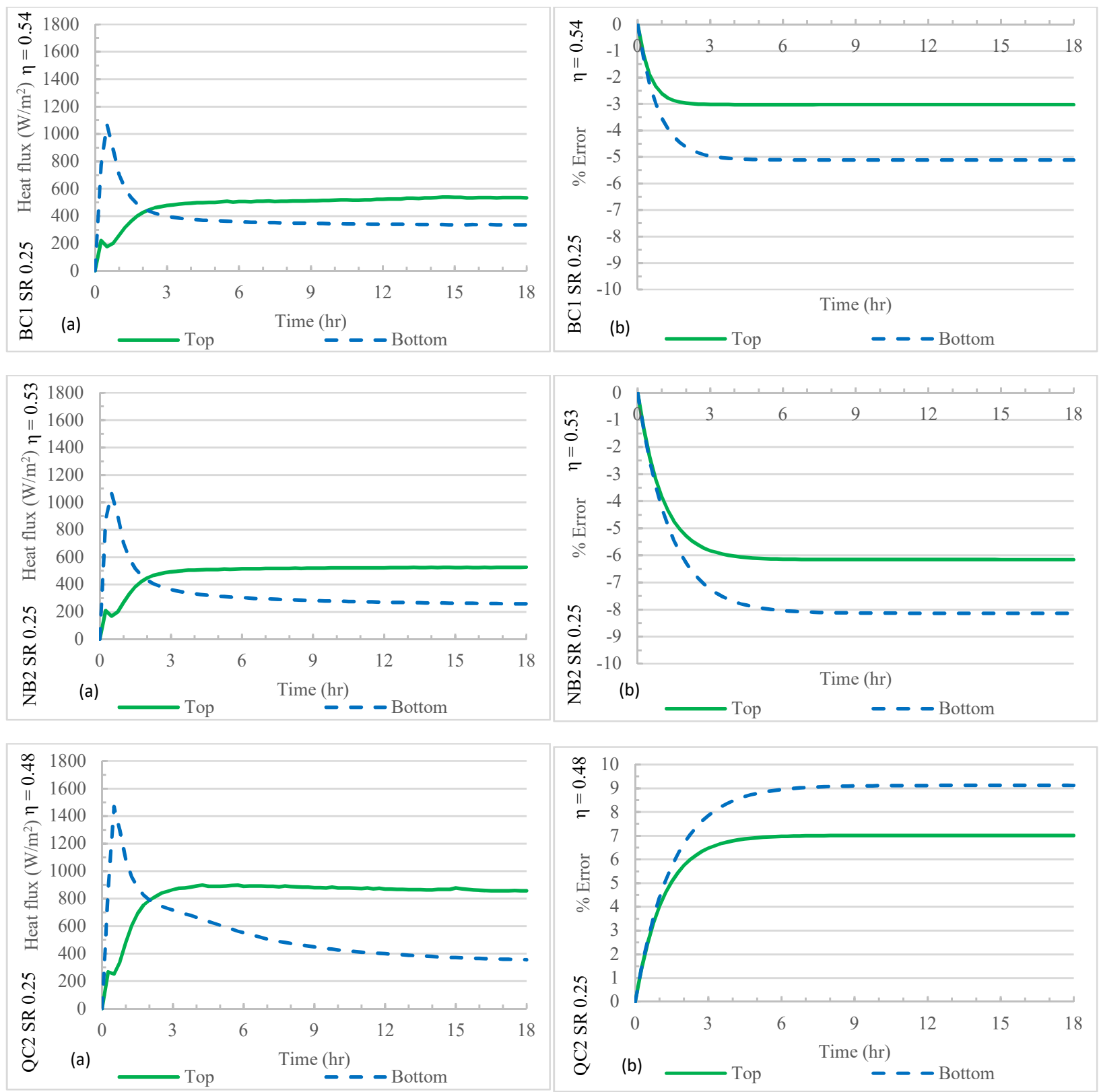

Figure E27. Simulated heat flux and \% error at top and bottom HFMs of wet soils $(S R \approx 0.25)$ and vertical heating from bottom. 

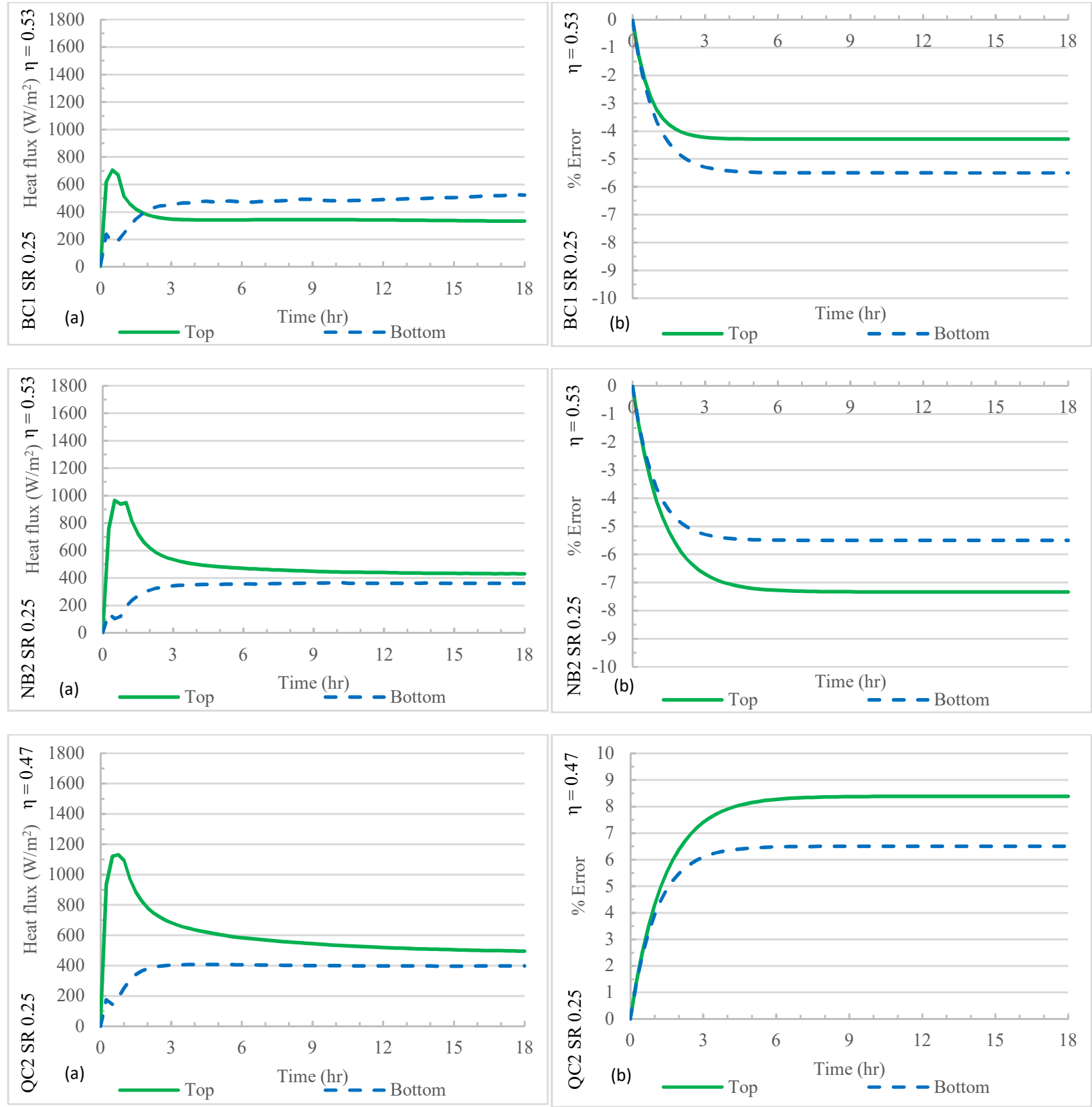

Figure E28. Simulated heat flux and \% error at top and bottom HFMs of wet soils $(S R \approx 0.25)$ and horizontal heating. 

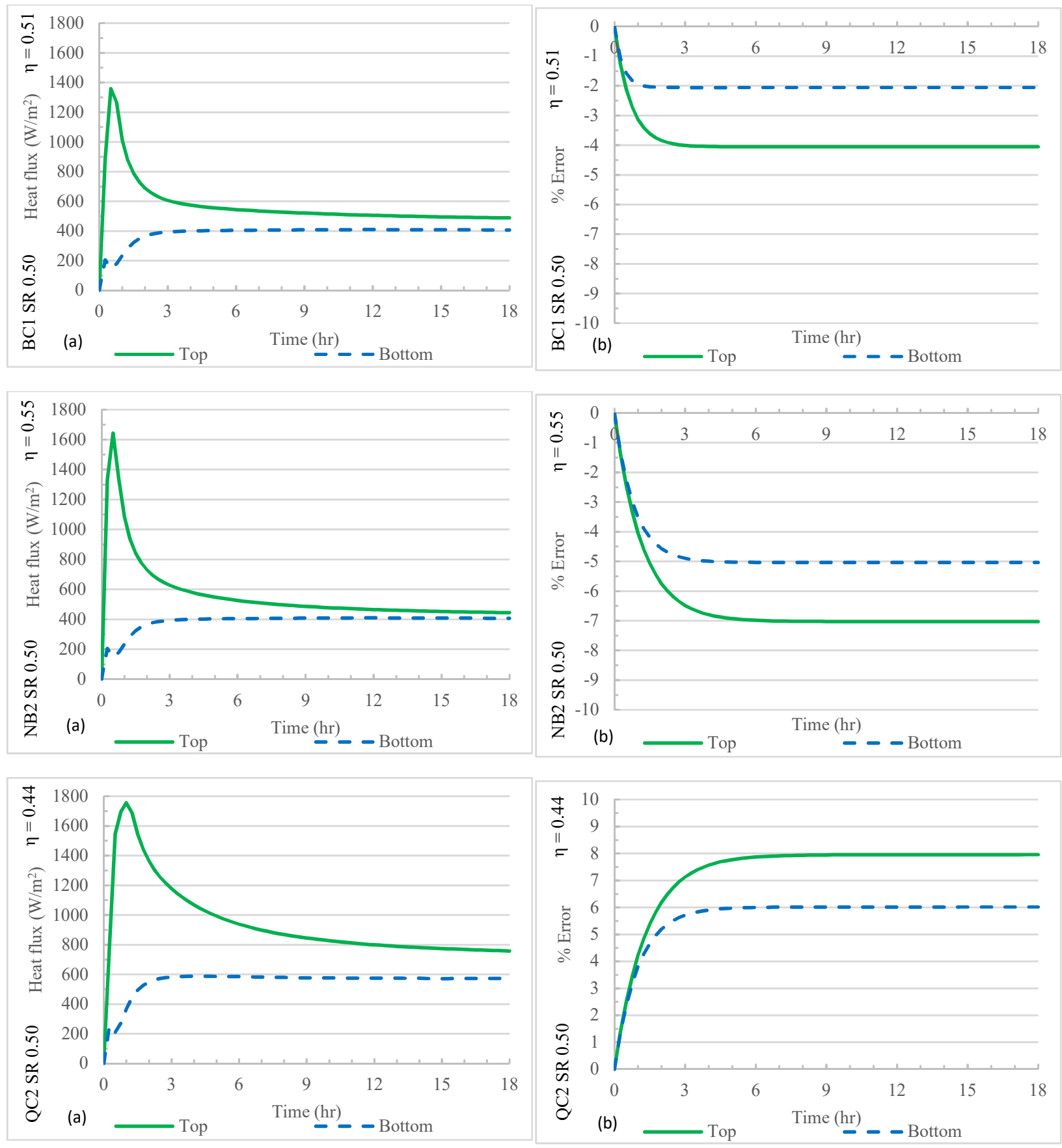

Figure E29. Simulated heat flux and \% error at top and bottom HFMs of wet soils $(S R \approx 0.50)$ and vertical heating from top. 

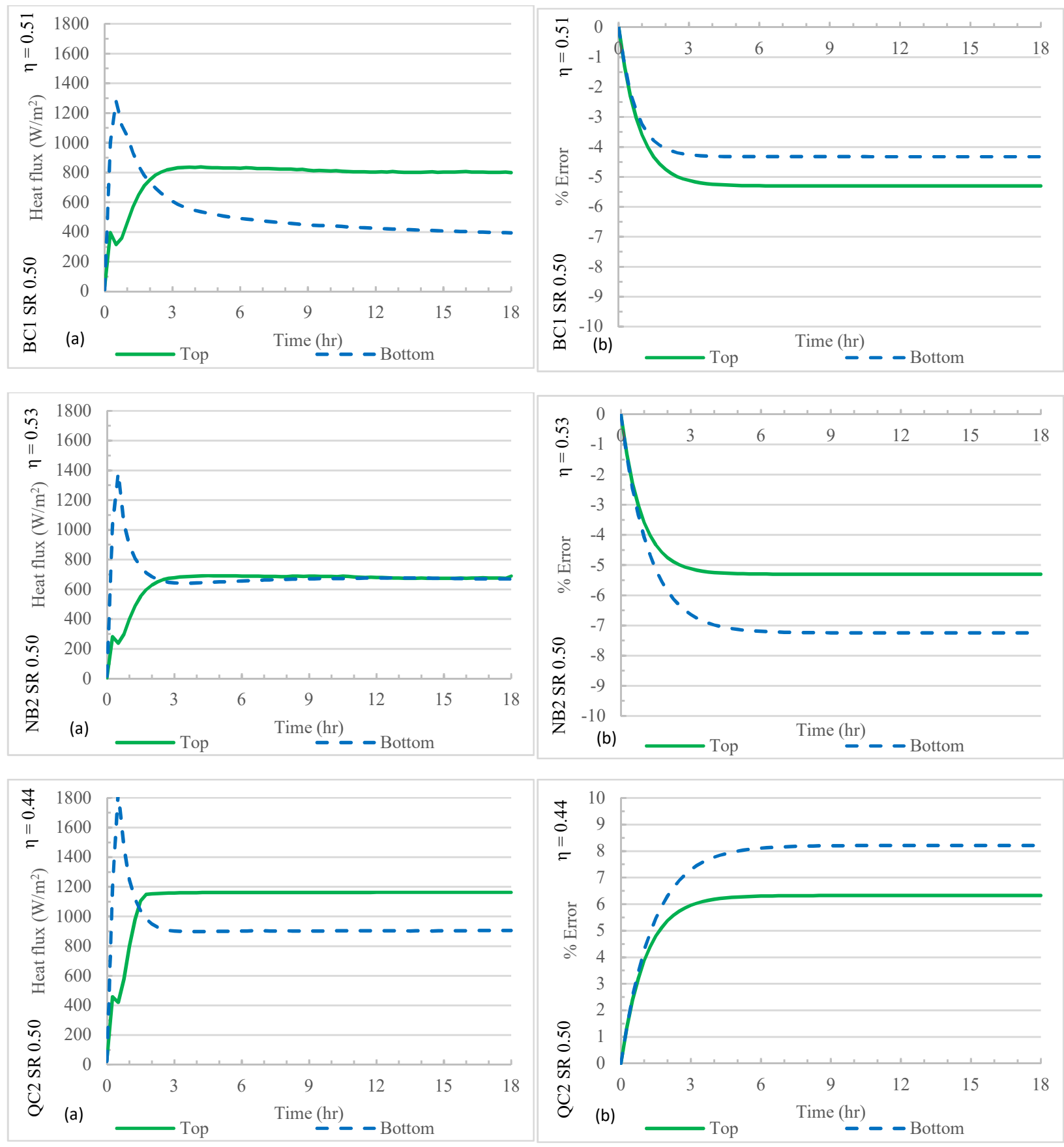

Figure E30. Simulated heat flux and \% error at top and bottom HFMs of wet soils $(S R \approx 0.50)$ and vertical heating from bottom. 

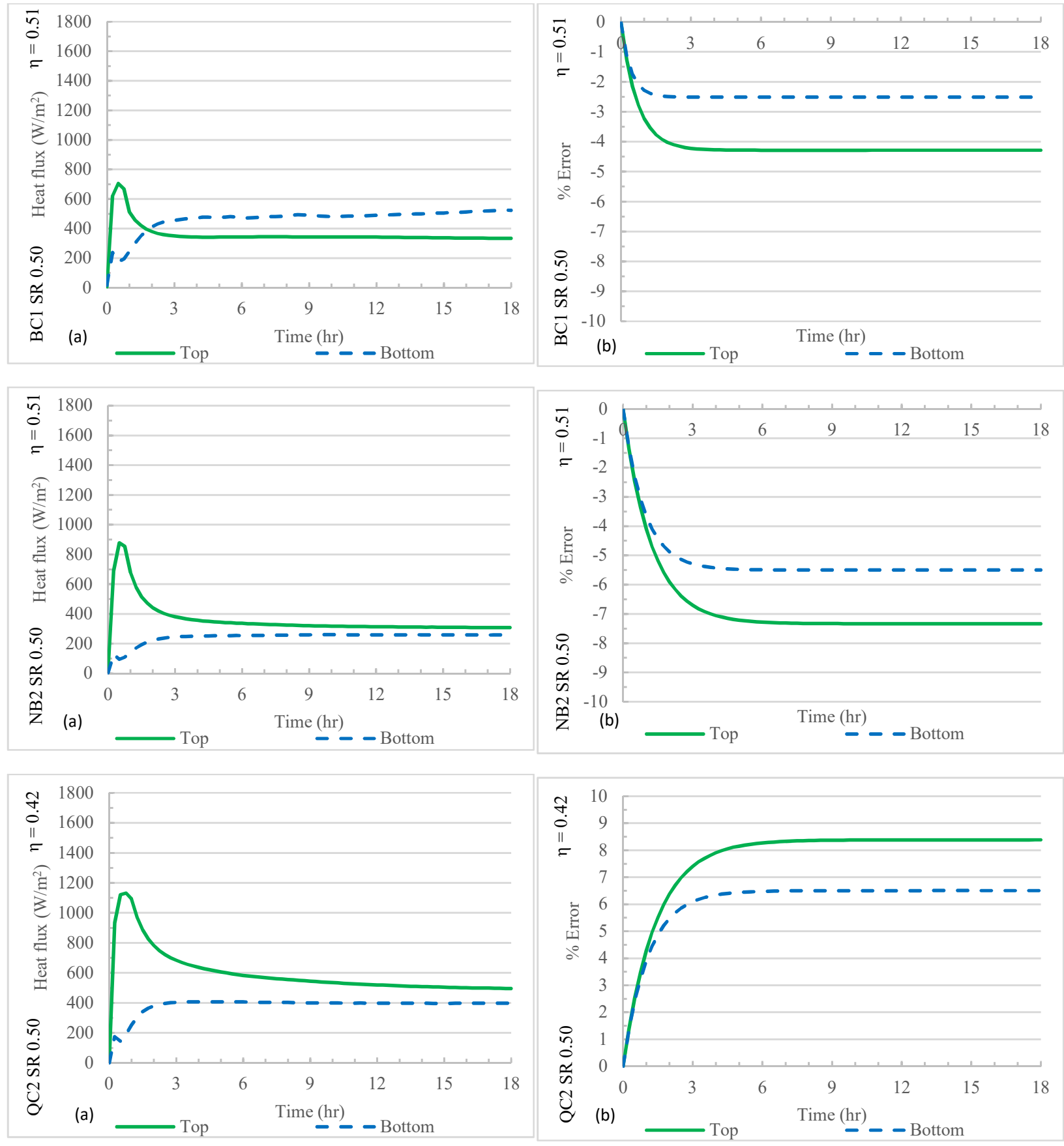

Figure E31. Simulated heat flux and \% error at top and bottom HFMs of wet soils $(S R \approx 0.50)$ and horizontal heating. 

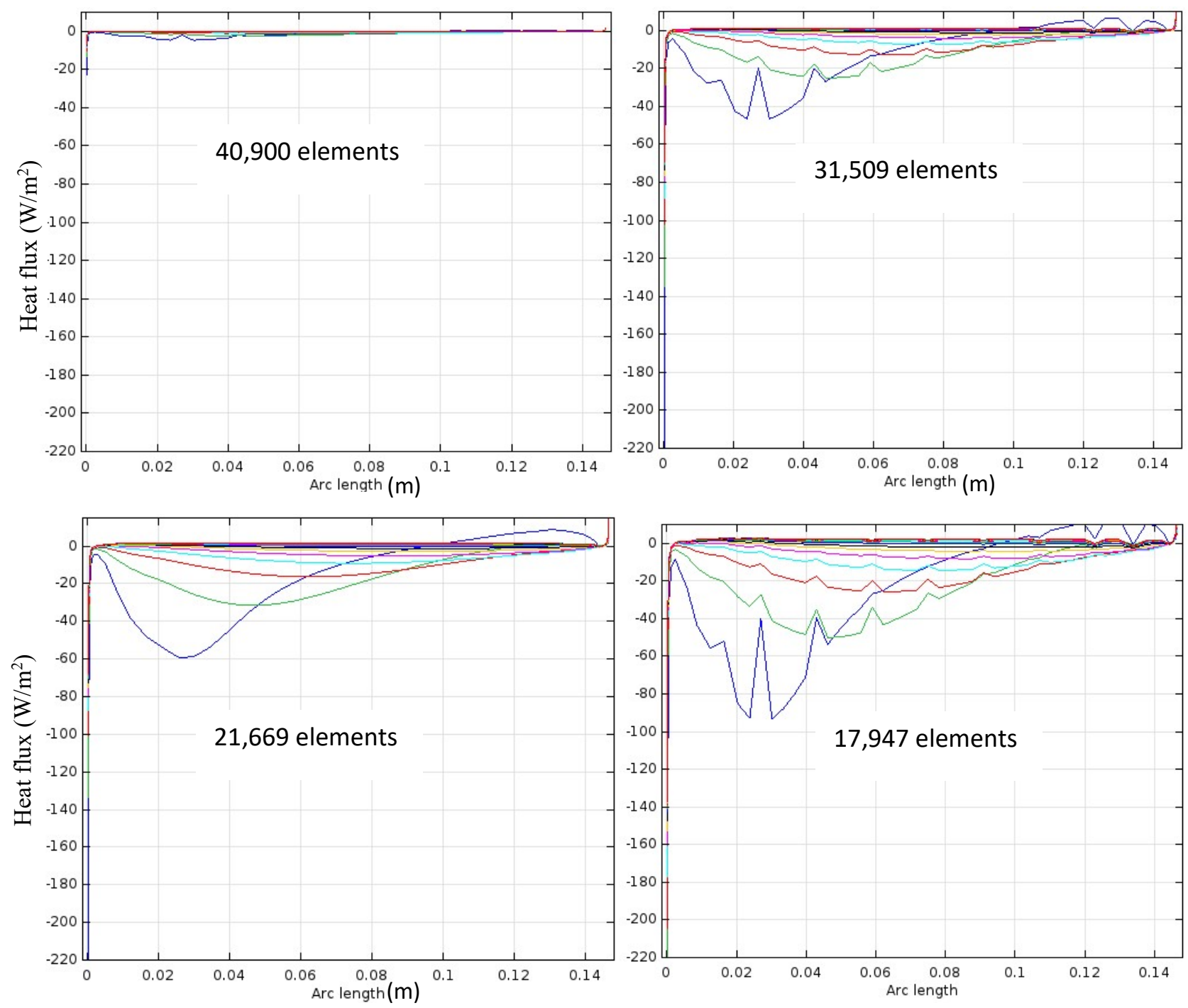

Figure E32. Centerline radial heat flux (for dry Matilda) from COMSOL using different number of elements. 


\section{F-Other Auxiliary Pictures}

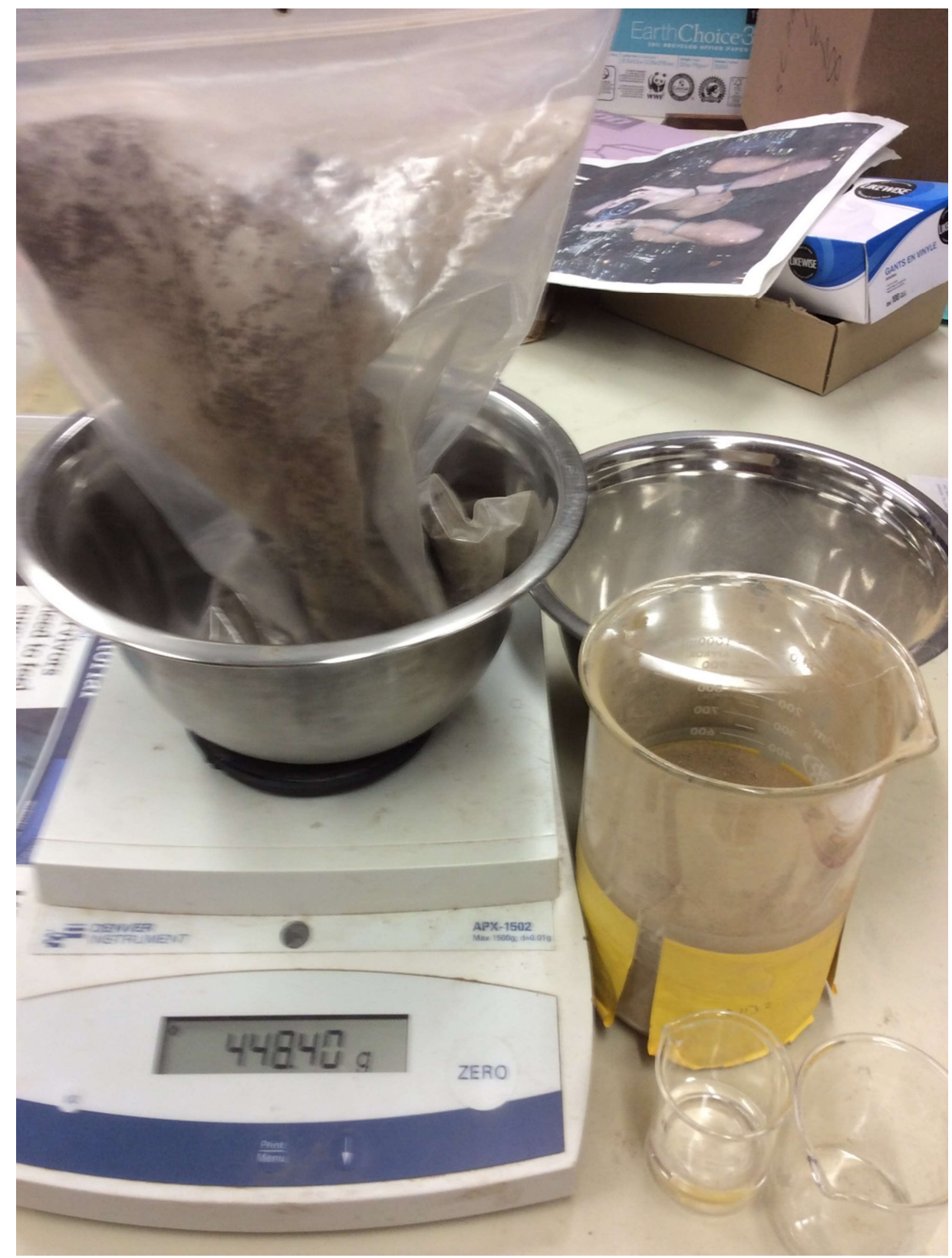

Figure F1. Sample of soil preparation before compaction into the soil column. 


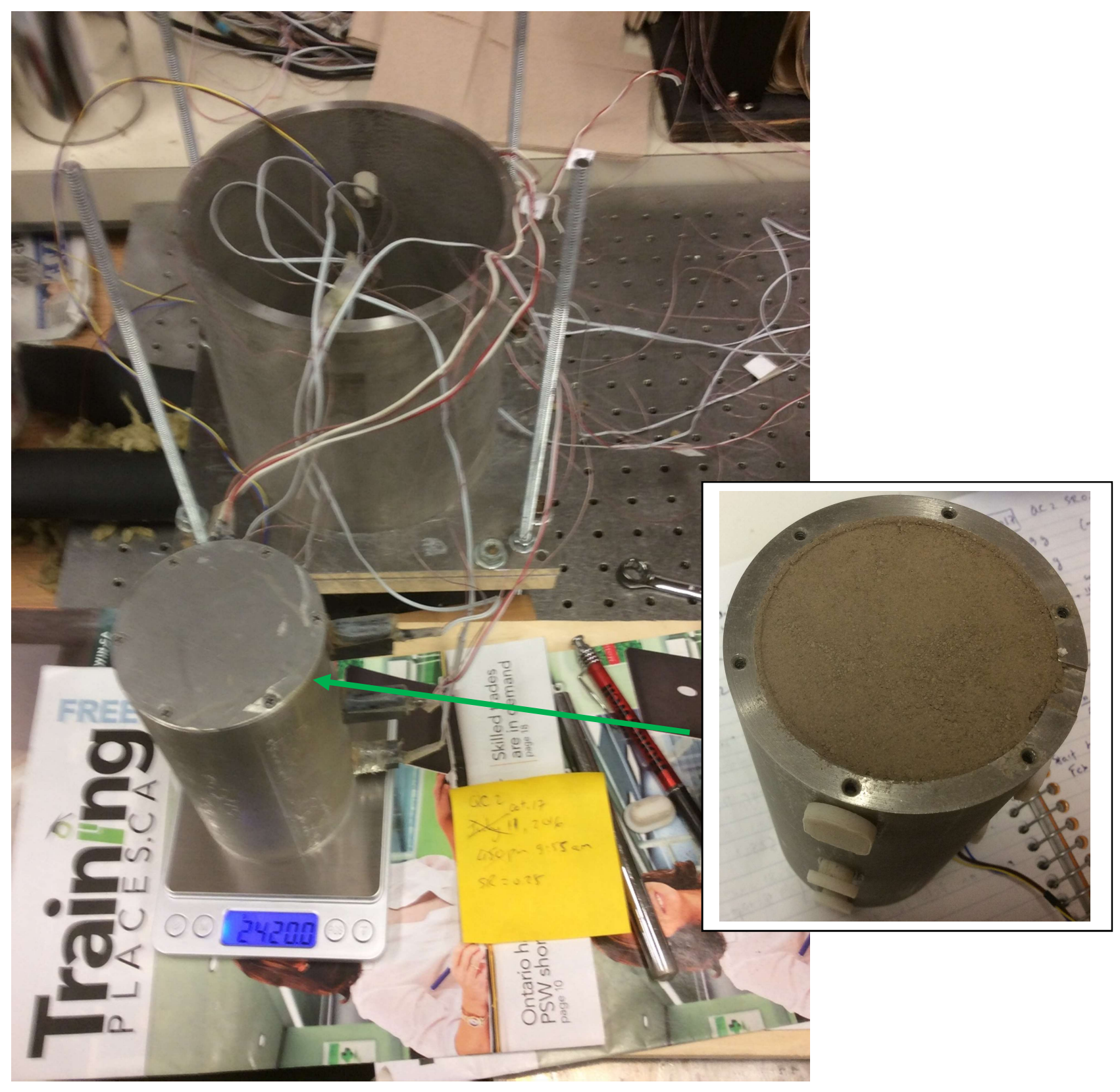

Figure F2. Sample of soil compaction. 


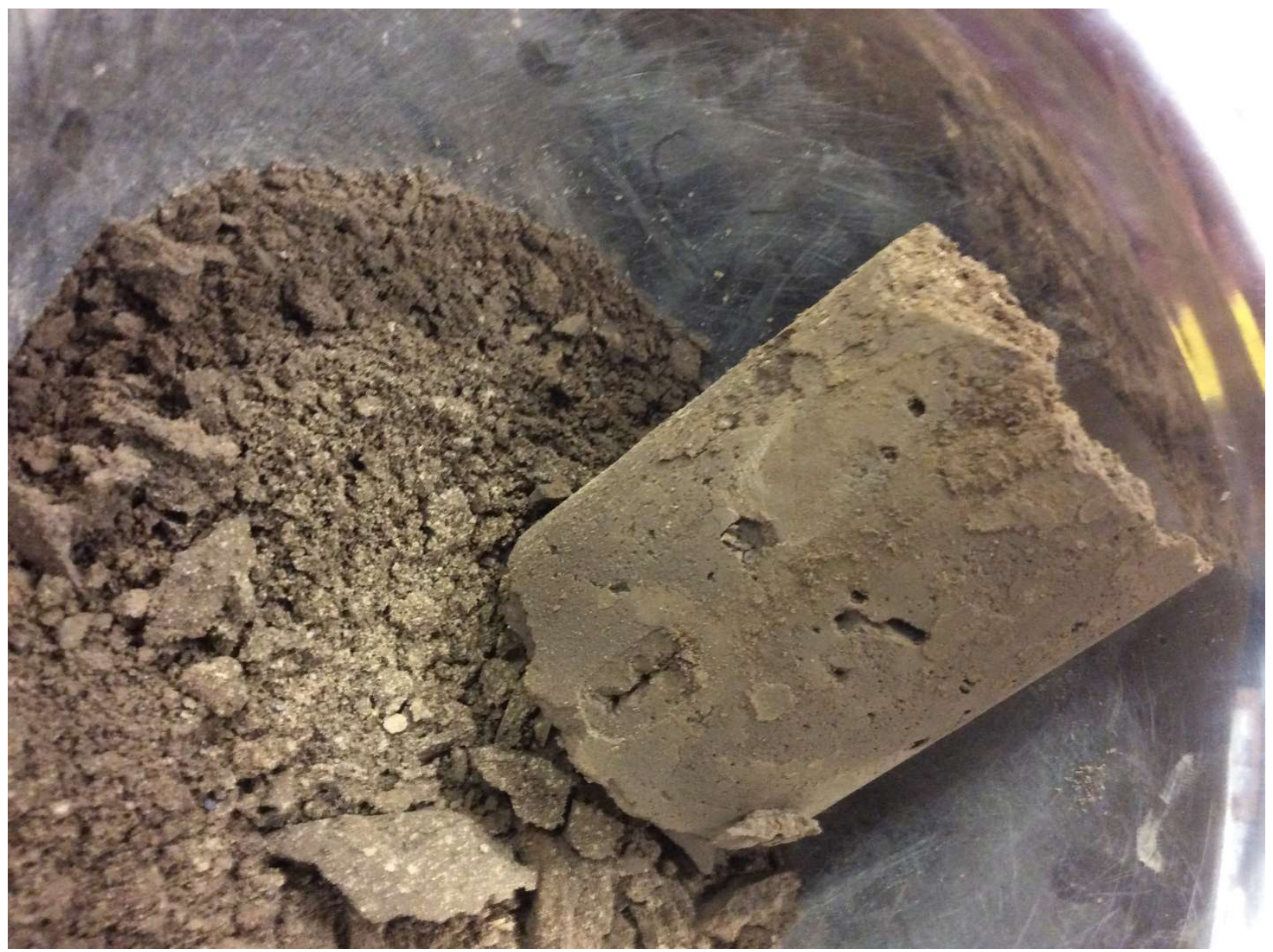

Figure F3. Sample of soil removal after an experiment. 


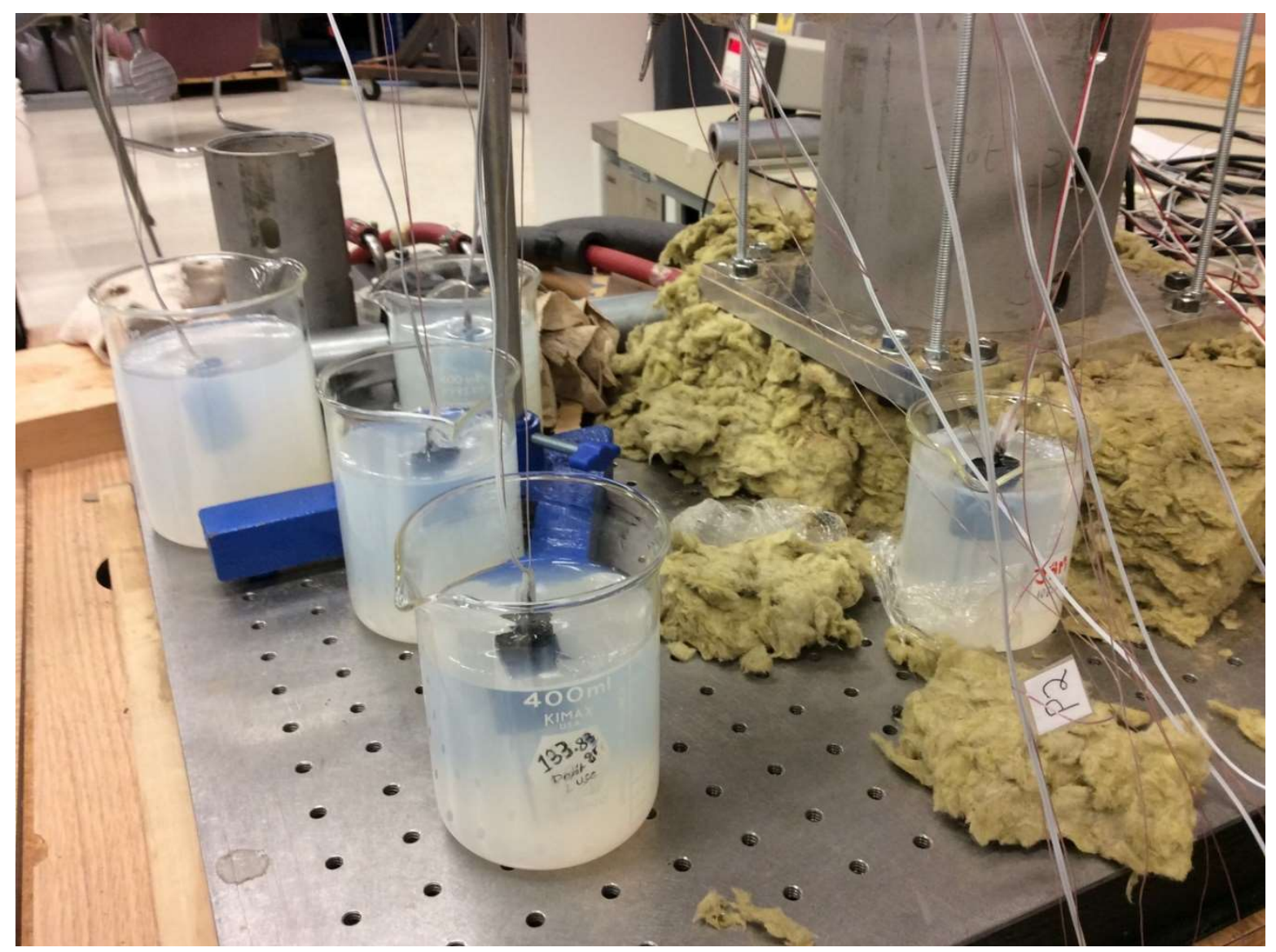

Figure F4. Setup for calibrations of probes which are immersed in a solution with 1\% agar and 99\% water. 


\section{REFERENCES}

[1] I.-I. Lin, I.F. Pun, C.-C. Lien; “Category-6” Super Typhoon Haiyan in Global Warming

Hiatus: Contribution from Subsurface Ocean Warming; AGU Geophysical Research Letters, pp. $8547-8553 ; 2014$.

[2] G. J. Boer; The Ratio of Land to Ocean Temperature Change under Global Warming; Clim. Dyn. Vol. 37, pp. 2253-2270; 2011.

[3] W. Yu, K. Mengersen, W. Hu, Y. Guo, X. Pan, S. Tong; Assessing the Relationship between Global Warming and Mortality: Lag Effects of Temperature Fluctuations by Age and Mortality Categories; Environmental Pollution, Vol. 159, pp. 1789 - 1793; 2011.

[4] International Federation of Red Cross and Red Crescent Societies, World Disaster Report, [Ch. 2]; IFRC; 2004.

[5] T. Kosatsky; The 2003 European Heat Waves; Euro Surveillance, 10(7), July 2005; http://eurosurveillance.org/en/v10n07/ 1007-222.asp

[6] J. Luterbacher, D. Dietrich, E. Xoplaki, M. Grosjean, H. Wanner; European Seasonal and Annual Variability, trends, and Extremes Since 1500; Science Vol. 303, pp. 1499 - 1503; 2004.

[7] A. Costello, M. Abbas, A. Allen, S. Ball, R. Bellam, et al.; Managing the Health Effect of Climate Change; The Lancet, Vol. 373, pp. 1693 - 1733; 2009.

[8] E. Massad, F. A. B. Coutinho, L. F. Lopez, D. R. da Silva; Modeling the Impact of Global Warming on Vector-Borne Infection; Physics of Life Reviews, pp. 169- 199; 2011.

[9] V. Penteriani, M. del Mar Delgado, H. Lokki; Global Warming May Depress Avian Population Fecundity by Selecting Against Early-Breeding High-Quality Individuals in Northern Populations of Sing-Brooded, Long-Lived Species; Annales Zoologici Fennici, Vol. 51, Issue 4, pp. 390 - 398; 2014.

[10] J. C. Habel, D. Rodder, T. Schmitt, G. Neves; Global Warming Will Affect the Genetic Diversity Uniqueness of Lycaena Helle Populations; Global Change Biology Vol. 17, pp. 194 205; 2011. 
[11] Y. Ramirez-amezcua, V. W. Steinmann, E. Ruiz-Sanchez, O. R. Rojas-Soto; Mexican Alpine Plants in the Face of Global Warming: Potential Extinction within a Specialized Assemblage of Narrow Endemics; Biodivers. Conserv. Vol. 25, pp. 865-885; 2016.

[12] http://theearthproject.com/fossil-fuels-bad-environment/, retrieved Dec. 22, 2016.

[13] Richard Matthews, http://www.thegreenmarketoracle.com/2015/03/infographic-how-to-endfossil-fuels-by.html, The Green Market Oracle, retrieved Dec. 22, 2016

[14] J. W. Cary; Soil Moisture Transport due to Thermal Gradients: Practical Aspects; Soil Science Society of America Proceedings, Vol. 30, No. 4, pp. 428 - 433; 1966.

[15] G. A. Martynov; Heat and Mass Transfer in Capillary-Porous Bodies, in Principles of Geocryology; National Research Council of Canada, Technical Translation 1065, Chapter 5; 1959.

[16] D. A. de Vries; A Nonstationary Method for Determining Thermal Conductivity of Soil in situ; Soil Science, Vol. 73, pp. 83 - 89; 1952.

[17] O. Johansen; Thermal Conductivity of Soils; Coprs. of Engineers, US Army (Draft translation 637, ADA044002); 1977.

[18] J. R. Philip, D. A. de Vries; Moisture Movement in Porous Materials under Temperature Gradients; American Geophysical Union Transaction, Vol. 38 (2), pp. 222 - 232; 1957.

[19] A. V. Luikov; Heat and Mass Transfer in Capillary-Porous Bodies (in "Advances in Heat Transfer”, edited by T. F. Irvine Jr. and J. P. Hartnett, pp. 123 - 184); 1964.

[20] H. R. Thomas, S. D. King; Coupled Heat and Mass Transfer in Unsaturated Soil. A Potential Based Solution; International Journal for Numerical and Analytical Methods in GeoMechanics, Vol. 16, pp. 757 - 773; 1992.

[21] M. Orme; Estimates of the Energy Impact of the Ventilation and Associated Financial Experience; Energy and Buildings, 33, pp. 199-255, 2001.

[22] P. Champsour; Recensement de la Population 1999 - Des logement Plus Grands et Plus Comfortables; INSEE (Institut de Statistique et des Etudes Economiques), p. 4, 2000, ISSN 09973192. 
[23] H. Wang, C. Qi, E. Wang, J. Zhao; A Case Study of Underground Thermal Storage in a Solar-Ground Coupled Heat Pump System for Residential Buildings; Renewable Energy, 34, pp.307-314; 2009.

[24] G. Oliveti, N. Arcuri, S. Ruffolo; Effect of Climatic Variability on the Performance of Solar Plants with inter-Seasonal Storage; Renewable Energy, 19(1), pp. 235-241; 2000.

[25] O. Ozgener, A. Hepbasli; Experimental Performance Analysis of a Solar Assisted GroundSource Heat Pump Greenhouse Heating System; Energy and Buildings, 37(1), pp. 101-110; 2005.

[26] Y. Weibo, S. Mingheng, D. Hua; Numerical Simulation of the Performance of a SolarEarth Source Heat Pump System, Applied Thermal Engineering, 26(17), pp. 2367-2376; 2006.

[27] M. Hedayati-Dezfooli; Development of an Experimental Apparatus for Studying HighTemperature Heat and Mass Transfer in Soils (PhD thesis); Ryerson University, 350 Victoria St., Toronto, Ontario, Canada, M5B2K3; 2015.

[28] M. Deru; A Model for Ground-Coupled Heat and Moisture Transfer from Buildings; Technical report (NREL/TP-550-33954, Contract No. DE-AC36-99-GO10337), National Renewable Energy Laboratory, 1617 Cole Boulevard, Golden, Colorado 80401-3393, USA; 2003.

[29] O. T. Farouki; Thermal Properties of Soils; CRREL Monograph 81-1; United States Army Corporations of Engineers, Cold Regions Research and Engineering Laboratory, New Hampshire, USA, 1981.

[30] I. Nikolaev, An Experimental Study of Soil Thermal Conductivity Using a Guarded Hot Plate Apparatus; MASc Thesis, Ryerson University, pp. 61-62, 2007, Call \#: QC323.N55 2007.

[31] C. G. Gurr, T. J. Marshall, T. J. Hutton; Movement of Water in Soil due to a Temperature Gradient; Soil Science, 74, pp. 335-345, 1952.

[32] S. A. Taylor, L. Cavazza; The Movement of Soil Moisture in Response to Temperature Gradients; Proceedings of Soil Science Society of America, 18, pp. 351-358, 1954.

[33] B. V. Derjaguin, M. K. Melnikova; Mechanics of Moisture Equilibrium and Migration in Soils; Water Conductivity in Soils, pp. 43-54, An internal symposium (Highway research board special report, no. 40), 1958.

[34] S. A. Taylor, J. W. Cary; Soil - Water Movement in Vapor and Liquid Phases; Methodology of Plant Eco-Systems, UNESCO Arid Zone Research, 25, pp. 159-165, 1965.

[35] B. J. Demsey; A Mathematical Model for Predicting Coupled Heat and Water Movement in Unsaturated Soil; International Journal for Numerical and Analytical Methods in Geomechanics, 2, pp. 19-34, 1978. 
[36] L. S. Shen; An Investigation of Transient, Two-Dimensional Coupled Heat and Moisture Flow in Soils; PhD Thesis, University of Minnesota, US, 1986.

[37] S. W. Rees, Z. Zhou, H. R. Thomas; The Influence of Soil Moisture Content Variations on Heat Losses from Earth-Contact Structures: An Initial Assessment; Building and Environment, 36, pp. 157-165, 2001.

[38] H. Janssen, J. Carmeliet, H. Hens; The Influence of Soil Moisture in the Unsaturated Zone on the Heat Loss from Buildings via the Ground; Journal of Thermal Environment \& Building Science, 25(4), pp. 275-298, 2002.

[39] M. Hornet, D. D. C. Nastac, I. L. Cirstolovean, N. Iordan, B. L. Maria, P. Mizgan, Ground Coupled Heat and Moisture Transfer from Buildings Basement; Recent Advances in Environmental Science, pp. 139 - 143, 2013.

[40] J. S. Marshall, A. P. Fuhrmann, Effect of Rainfall Transients on Thermal and Moisture Exposure of Underground Electric Cables; International Journal of Heat and Mass Transfer, 80, pp. 660-672, 2015.

[41] G. C. Topp, J. L. Davis, A. P. Annan; Electromagnetic Determination of Soil Water Content: Measurements in Coaxial Transmission Lines; Water Resources, Vol. 16, pp. 574 - 582; 1980.

[42] T. Ren, K. Noborio, R. Horton; Measuring Soil Water Content, Electrical Conductivity, and Thermal Properties with a Thermo-Time Domain Reflectometry Probe; Soil Soc. Am. J. Vol. 63, pp. $450-457 ; 1999$.

[43] R. Horton, T. Ren, Z. Ju, Y. Gong; Comparing Heat-Pulse and Time Domain Rerflectometry Soil Water Contents from Thermo-Time Domain Reflectometry Probes; Vadose Zone Journal Vol. 4, pp. 1080 - 1086; 2005.

[44] J. L. Heitman, R. Horton, T. Ren, T. E. Ochsner; An Improved Approach for Measurement of Coupled Heat and Water Transfer in Soil Cells; Journal of Soil Physics Vol. 71; 2007.

[45] N. Zhang, X. Yu, and A. Pradhan; Application of a thermo-time domain reflectometry probe in sand-kaolin clay mixtures; Engineering Geology, Vol. 216, pp. 98-107; 2017.

[46] B. Sibbitt, T. Onno, D. McClenahan, J. Thornton, A. Brunger, J. Kokko, B. Wong; The Drake anding Solar Community Project - Early Results; Joint SESCI \& Solar Buildings Networks Conference, 2007.

[47] B. Sibbitt, D. McClenahan, R.Djebbar, J. Thornton, B. Wong, J. Carriere, J. Kokko; The Performance of a High Solar Fraction Seasonal Storage District Heating System - Five Years of Operation; Energy Procedia, 30, pp. 856-865, 2012. 
[48] V. Lottner, M. E. Schulz, E. Hahne; Solar-Assisted District Heating Plants: Status of the German Programme Solarthermie-2000; Solar Energy, 69(6), pp. 449-459, 2000.

[49] M. Reuss, M. Beck, J. P. Muller; Design of a Seasonal Thermal Energy Storage in the Ground; Solar Energy, 59(4-6), pp. 247-257, 1997.

[50] A. Gabrielsson, U. Bergdahl, L. Moritz; Thermal Energy Storage in Soils at Temperatures Reaching 90 $^{\circ}$; Journal of Solar Energy Engineering, 112, pp. 3-8, 2000.

[51] M. T. van Genuchten; A Close-Form Equation for Predicting the Hydraulic Conductivity of Unsaturated Soils; Soil Science Society of America Journal Vol. 44, pp. 892 - 898; 1980.

[52] C. D. Grant, P. H. Groenevelt, N. I. Robinson; Application of the Groenevelt-Grant Soil Water Retention Model to Predict the Hydraulic Conductivity; Australian Journal of Soil Research Vol. 48, pp. 447 - 458; 2010.

[53] D. A. de Vries; Physics of the Plant Environment, Van Wijk W. R. (eds); North-Holland, Amsterdam; 1966.

[54] J. H. Knight, G. J. Kluitenberg, T. Kamai, J. W. Hopman; Semianalytical Solution for DualProbe Heat-Pulse Applications that Accounts for Probe Radius and Heat Capacity; Vadose Zone J., Vol. 11, Issue 2; 2012.

[55] ISO; Guide to the Expression of Uncertainty in Measurement; ISO Geneva 1995.

[56] G. J. Kluitenberg, K. L. Bristow, B. S. Das; Error Analysis of Heat Pulse Method for Measuring Soil Heat Capacity, Duffusivity, and Conductivity; Department of Agronomy, Kansas State University, Manhattan, KS 66506 CSIRO Division of Soils, PMB, PO Aitkenvale, Townsville, QLD 4814, Australia, Vol. 59, pp. 719 - 726; 1995.

[57] V. R. Tarnawski, T. Momose, M. L. McCombie, W. H. Leong; Canadian Field Soils III. Thermal-Conductivity Data and Modeling; Int. J. Thermophys. Vol. 36, pp. 119 - 156; 2015.

[58] J. Frost; Regression Analysis: How Do I Interpret R-squared and Access the Goodness-offit?, from http://blog.minitab.com/blog/adventures-in-statistics-2/regression-analysis-how-do-iinterpret-r-squared-and-assess-the-goodness-of-fit; 2013.

[59] L. Dang, W. H. Leong; Thermal Conductivity Probe - Part II - An Experimental Analysis; International Journal of Heat and Mass Transfer, pp. 1004 - 1014; 2015.

[60] H. K. Versteeg, W. Malalasekera; An Introduction to Computational Fluid Dynamics - The Finite Volume Method, $2^{\text {nd }}$ Ed.; Pearson Education Ltd., Edinburg Gate, Harlow, Essex CM20 2JE, England; 2007. 
[61] J. Y. Murthy; Numerical Method in Heat, Mass, and Momentum Transfer (Draft Notes); School of Mechanical Engineering, Purdue University; 2002.

[62] K. C. Karki, S. V. Pantakar; Application of the Partial Elimination Algorithm for Solving the Coupled Energy Equations in Porous Media; Numerical Heat Transfer, Part A: Applications (45), pp. 539 - 549; 2004.

[63] F. Moukalled, S. Saleh; Heat and Mass Transfer in Moist Soil, Part I. Formulation and Testing; Numerical Heat Transfer, Part B, 49, pp. 467-486, 2006.

[64] F. P. Incropera, D. P. DeWitt; Fundamentals of Heat and Mass Transsfer; Wiley, New York; 2002.

[65] J. R. Philip; The Theory of Infiltration: 1. The Infiltration Equation and its Solution; Soil Science, Vol. 83, pp. 435 - 448; 1957.

[66] R. Haverkamp, M. Vauclin, J. Touma, P. J. Wierenga, G. Vachaud; Comparison of Numerical Simulation Models for One-Dimensional Infiltration; Soil Science Society of America Journal, Vol. 41, no. 2, pp. 285 - 294; 1977.

[67] J. R. Welty, C. E. Wicks, R. E. Wilson, G. L. Rorrer; Fundamentals of Momentum, Heat and Mass Transfer - $5^{\text {th }}$ Ed.; John Wiley \& Sons Inc., 111 River Street, Hoboken, NJ 070305774; ISBN: 978-0470128688; 2008.

[68] M. T. Van Genuchten, Close-Form Equation for Predicting the Hydraulic Conductivity of Unsaturated Soils; Soil Science Society of America Journal, 44(5), pp. 892-898, 1980.

[69] K. Noborio, K. J. McInnes, J. L. Heilman; Two-Dimensional Model for Water, Heat, and Solute Transport in Furrow-Irrigated Soil: II. Field Evaluation; Soil Science Society of America Journal (60), pp. 1001 - 1009; 1996.

[70] P. S. Eagleson; Dynamic Hydrology, New York: McGraw-Hill; 1970.

[71] P. C. D. Milly; A Simulation Analysis of Thermal Effects on Evaporation From Soil; Water Resources Research (20) 8, pp. 1087 - 1098; 1982.

[72] P. C. D. Milly; Moisture and Heat Transport in Hysteretic, Inhomogeneous Porous Media: A Matric Head-Based Formulation and a Numerical Model; Water Resources Research (18) 3, pp. 489 - 498; 1984.

[73] Department of Land, Air and Water Resources, University of California, Davis; http://lawr.ucdavis.edu/classes/ssc107/SSC107Syllabus/chapter1-00.pdf; retrieved July 18, 2016. 